\title{
AUTOMATIC RULE DISCOVERY AND GENERALIZATION IN SUPERVISED AND UNSUPERVISED LEARNING TASKS
}

\author{
By
}

Robert Cattral, B.C.S., M.C.S.

A thesis submitted to

The Faculty of Graduate Studies and Research

In partial fulfillment of

the requirements for the degree of

Doctor of Philosophy

Ottawa-Carleton Institute for Computer Science

\author{
School of Computer Science \\ Carleton University \\ Ottawa, Ontario
}

June 2008

(C) Copyright

2008, Robert Cattral 


$\begin{array}{ll}\begin{array}{l}\text { Library and } \\ \text { Archives Canada }\end{array} & \begin{array}{l}\text { Bibliothèque et } \\ \text { Archives Canada }\end{array} \\ \begin{array}{l}\text { Published Heritage } \\ \text { Branch }\end{array} & \begin{array}{l}\text { Direction du } \\ \text { Patrimoine de l'édition }\end{array} \\ \begin{array}{l}\text { 395 Wellington Street } \\ \text { Ottawa ON K1A 0N4 } \\ \text { Canada }\end{array} & \begin{array}{l}\text { 395, rue Wellington } \\ \text { Ottawa ON K1A 0N4 } \\ \text { Canada }\end{array}\end{array}$

Your file Votre référence ISBN: 978-0-494-43888-6 Our file Notre référence ISBN: 978-0-494-43888-6

NOTICE:

The author has granted a nonexclusive license allowing Library and Archives Canada to reproduce, publish, archive, preserve, conserve, communicate to the public by telecommunication or on the Internet, loan, distribute and sell theses worldwide, for commercial or noncommercial purposes, in microform, paper, electronic and/or any other formats.

The author retains copyright ownership and moral rights in this thesis. Neither the thesis nor substantial extracts from it may be printed or otherwise reproduced without the author's permission.
AVIS:

L'auteur a accordé une licence non exclusive permettant à la Bibliothèque et Archives Canada de reproduire, publier, archiver, sauvegarder, conserver, transmettre au public par télécommunication ou par l'Internet, prêter, distribuer et vendre des thèses partout dans le monde, à des fins commerciales ou autres, sur support microforme, papier, électronique et/ou autres formats.

L'auteur conserve la propriété du droit d'auteur et des droits moraux qui protège cette thèse. $\mathrm{Ni}$ la thèse ni des extraits substantiels de celle-ci ne doivent être imprimés ou autrement reproduits sans son autorisation.
In compliance with the Canadian Privacy Act some supporting forms may have been removed from this thesis.

While these forms may be included in the document page count, their removal does not represent any loss of content from the thesis.
Conformément à la loi canadienne sur la protection de la vie privée, quelques formulaires secondaires ont été enlevés de cette thèse.

Bien que ces formulaires aient inclus dans la pagination, il n'y aura aucun contenu manquant.

\section{Canada}




\section{Abstract}

Data Mining algorithms have been the focus of much research in recent years and new techniques are being developed regularly. This thesis describes EvRFind, an application for rule discovery in the task of Data Mining.

EvRFind is a hybrid Genetic Algorithm that also employs techniques from statistics and machine learning to improve efficiency and performance of the search. Among the non-evolutionary components are algorithms such as gradient ascent local search (Hill Climbing), optimization methods designed to improve search speed, automatic concept generalization, and automatic expansion of the description language.

EvRFind creates predictive models in the form of a default hierarchy. Each hierarchy is comprised of a set of rules that are ordered by generality, and selected with a bias towards minimum-length and comprehensibility.

Experiments on several datasets are run to evaluate EvRFind, and the results are compared to published work. To properly evaluate and illustrate the features and expressive power of EvRFind, the Poker Hand Dataset was created. This dataset represents a very large, imbalanced, and challenging domain. There are several target concepts, each with varying distribution within the dataset. The results achieved by EvRFind are compared to those generated by several other machine learning algorithms. 


\section{Acknowledgments}

I would like to start by thanking my thesis supervisor, Franz Oppacher, for the many years of support and guidance that he has provided. It is thanks to his courses on Artificial Intelligence that I was lead to specialize in the area of Genetic Algorithms. Combined with other aspects of machine learning, the Genetic Algorithm is the central component of this thesis.

The meetings of the Artificial Life and Evolutionary Computing group at Carleton University have been an important forum for the discussion of revolutionary ideas. Many insightful comments and suggestions have come from attendees and fellow graduate students including Mark Wineberg, Lee Graham, Steffen Christensen, Alex Tulai, Yandu Oppacher, Jacky Mallett, and Hassan Masum.

Special thanks also go to the members of my Ph.D. committee: Dwight Deugo, Stan Matwin, Shirley Mills, and Andrew Kusiak. The effort that each put into reading and making suggestions on my thesis is very much appreciated. In particular, I would like to thank my external examiner, Dr. Andrew Kusiak, for making the trip from the University of Iowa to be a part of my examining committee.

I would also like to thank the Computer Science department at Carleton University and in particular their administrative staff. It is the extra effort from people like Claire Ryan, Linda Pfeiffer, Sandy Herbert, and Sharmila Namasivayampillai that allows students to 
fulfill their requirements without having to micromanage every academic detail and deadline.

It is also important for me to thank my pre-university mentors and colleagues, Ray Novokowski, Jim Kirk, and Steve Fleury for their guidance and teachings that focused my interests and abilities at an early point in my career. Not to forget another important influence in the same circle of peers, Francesca Merlo. It was Francesca who first suggested that I return to academia with the goal of achieving a Doctorate degree in Computer Science.

Finally, I would like to acknowledge and thank the many people who have provided financial support or have otherwise encouraged my work over the past several years. I would like to thank Lee Graham and Anne Hargrave for lending their proofreading abilities at various times. I would also like to thank my mother, Anne Cattral, my friend Shaun Sauve who would constantly prod me about deadlines, and finally my business partner, Catherine Brunet. 


\section{Table of Contents}

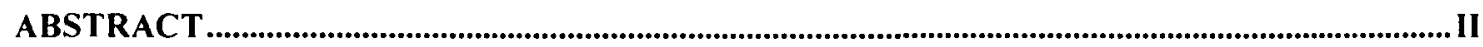

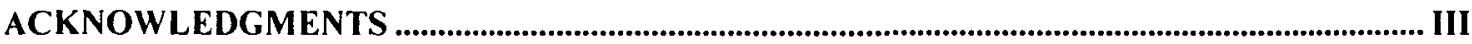

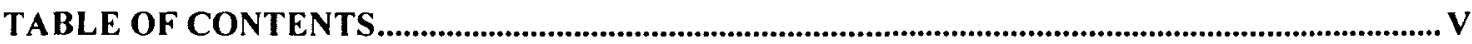

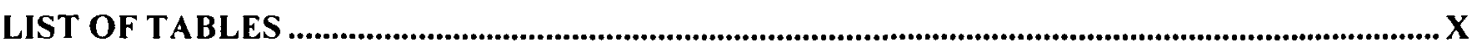

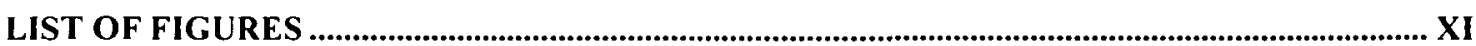

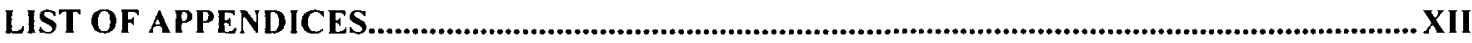

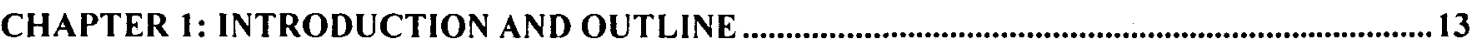

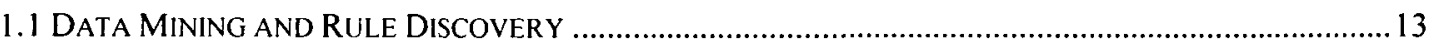

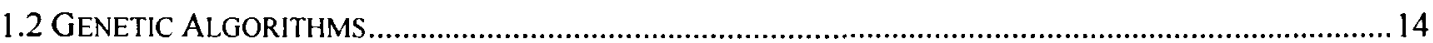

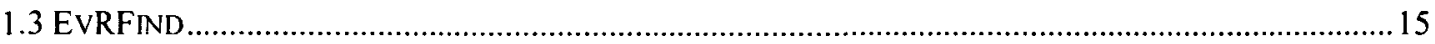

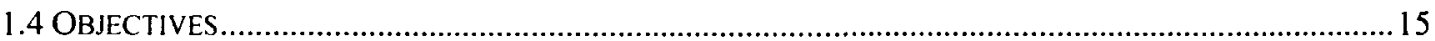

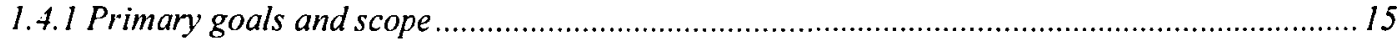

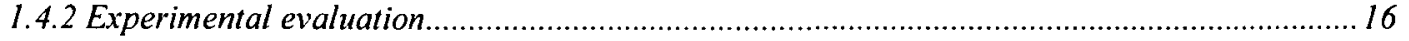

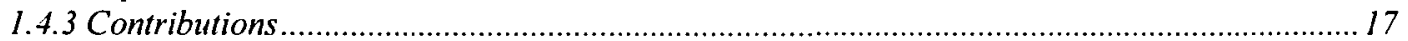

1.4.4 Summary of contributions and intermediate objectives …….............................................. 17

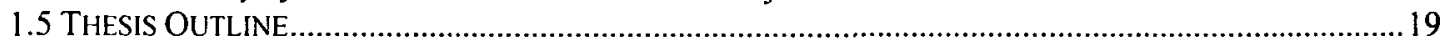

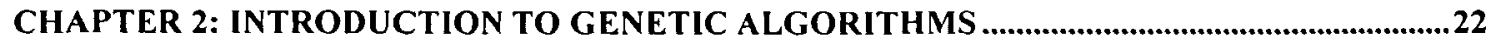

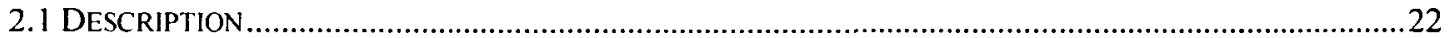

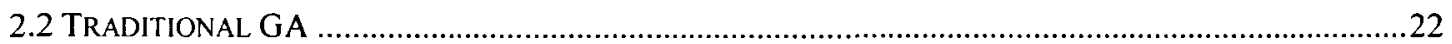

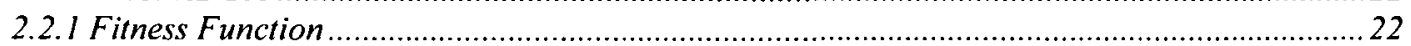

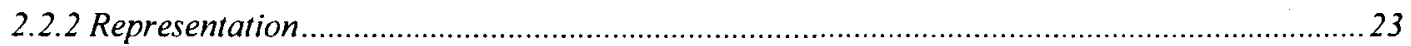

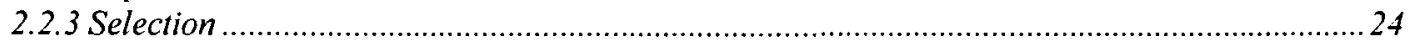

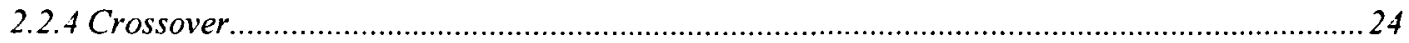

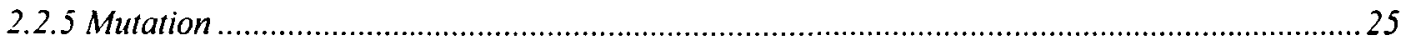

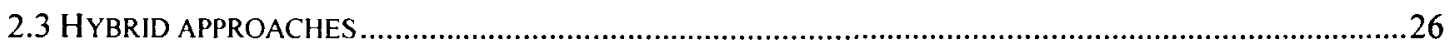

CHAPTER 3: DATA MINING - PAST AND PRESENT …...........................................................27

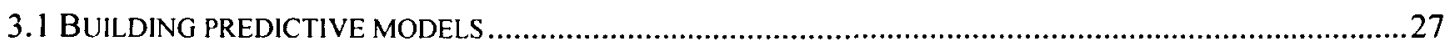

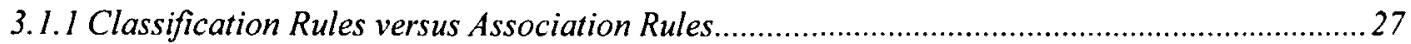

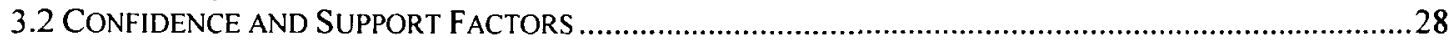

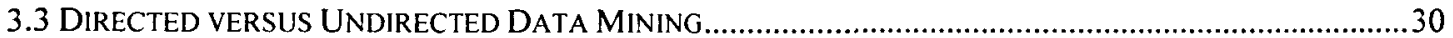

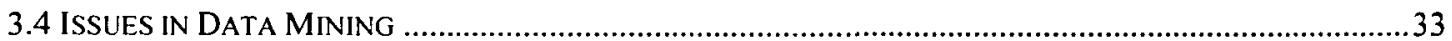

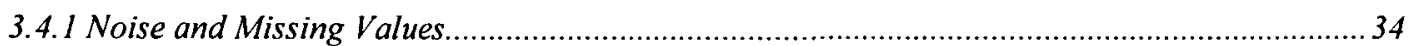

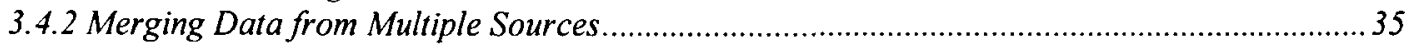

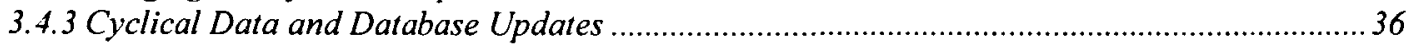

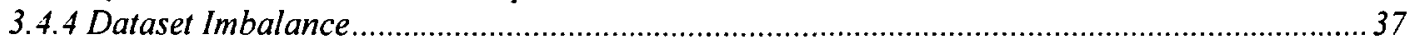

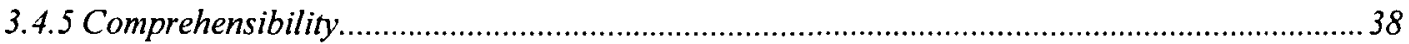

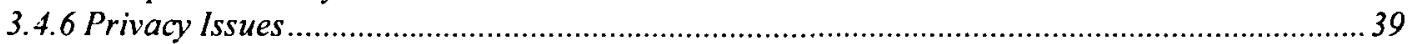

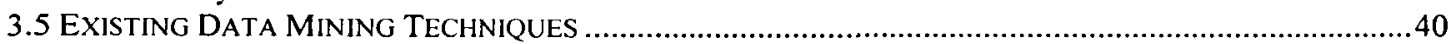

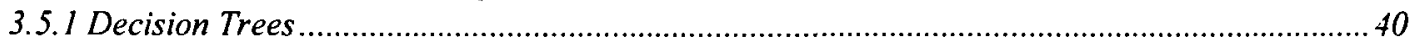

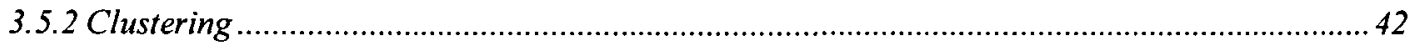

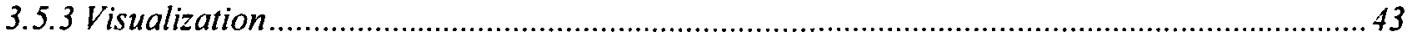

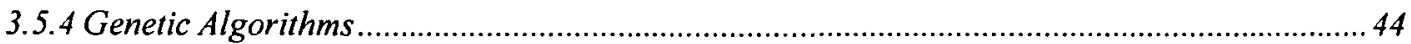

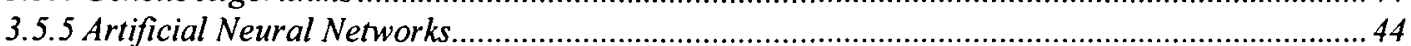

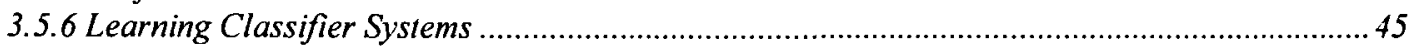

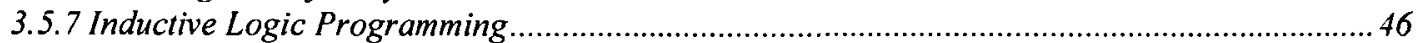

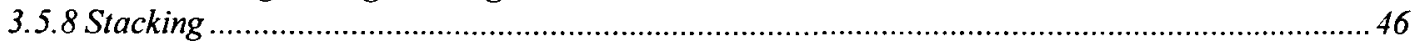




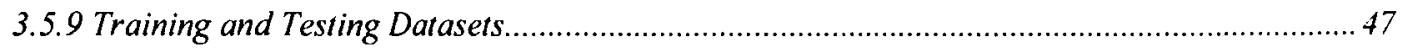

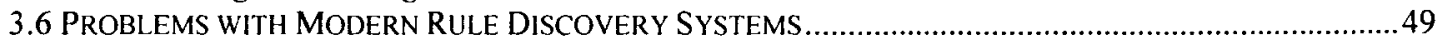

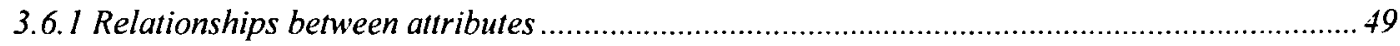

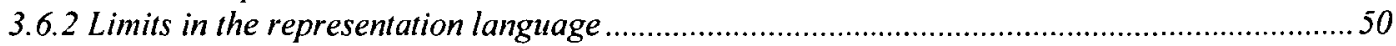

3.6.3 Building on previously discovered knowledge ...................................................................

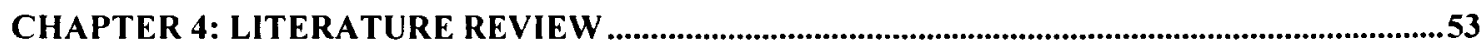

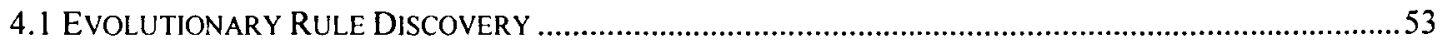

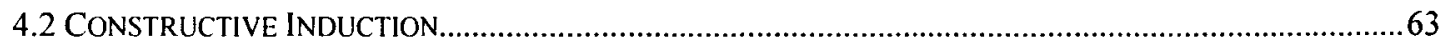

CHAPTER 5: EVRFIND - DETAILED DESCRIPTION .........................................................................67

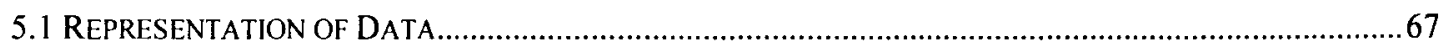

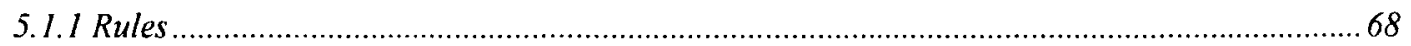

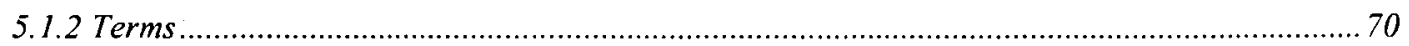

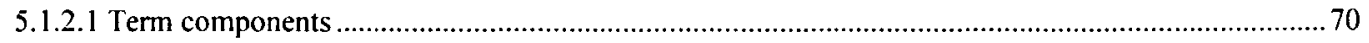

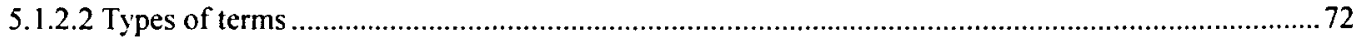

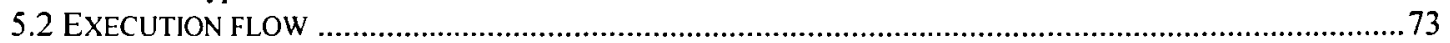

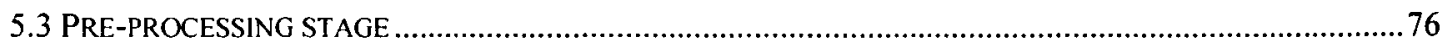

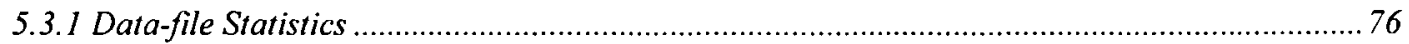

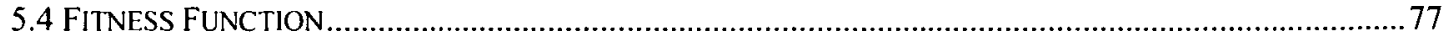

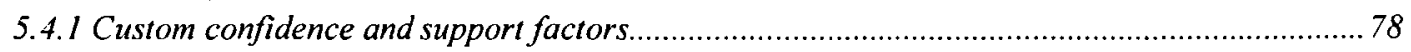

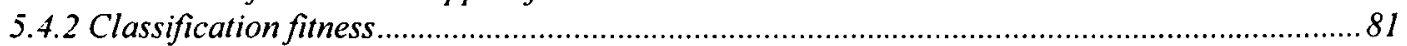

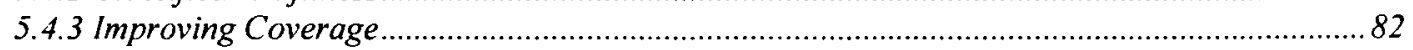

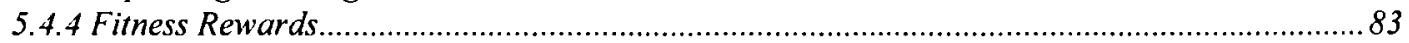

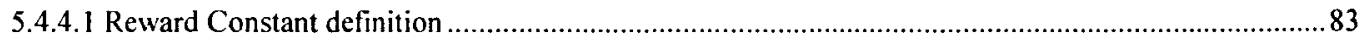

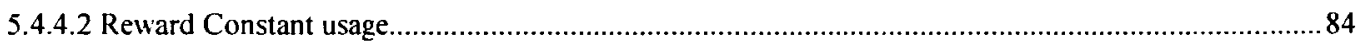

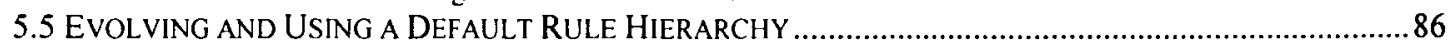

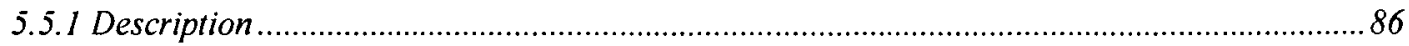

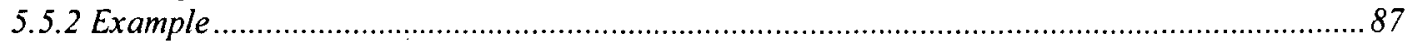

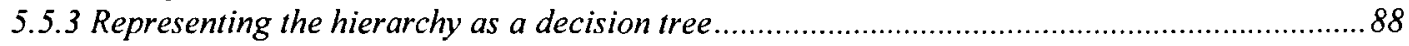

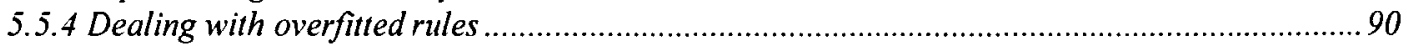

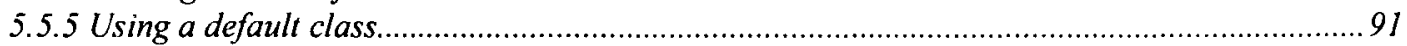

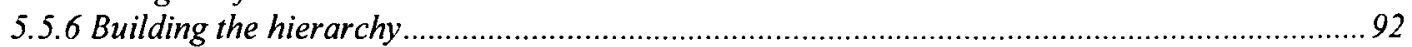

5.5.6.1 Global Fitness versus Hierarchy Fitness.........................................................................................92

5.5.6.2 Method for specifically trying to induce the default hierarchy …….............................................95

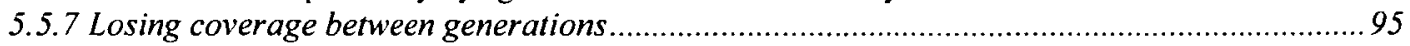

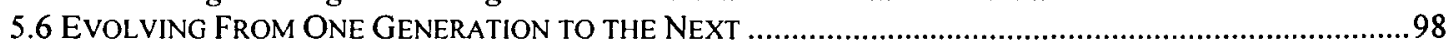

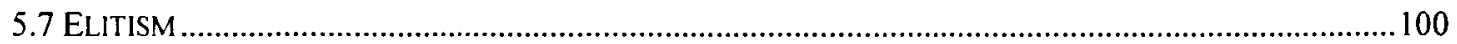

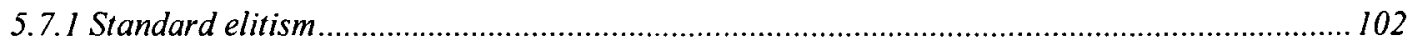

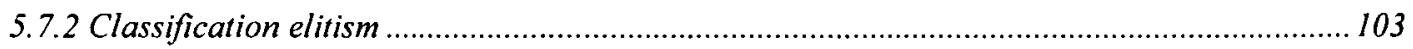

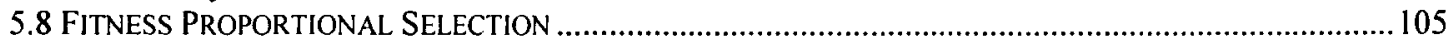

5.9 CROSSOVER

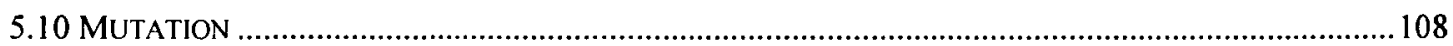

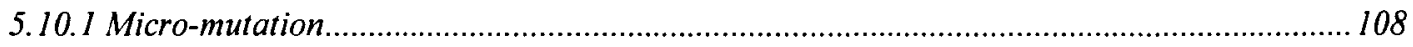

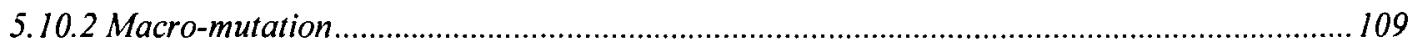

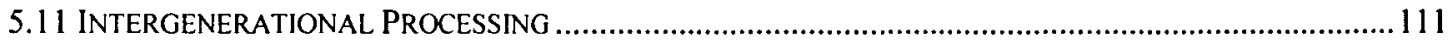

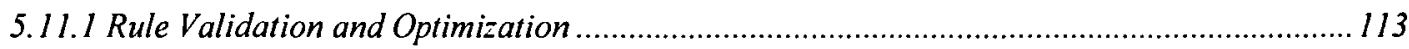

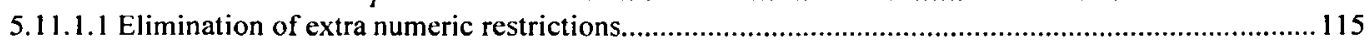

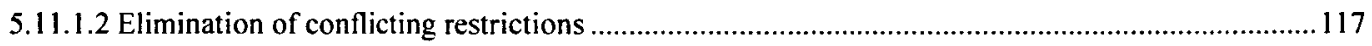

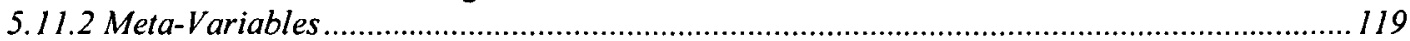

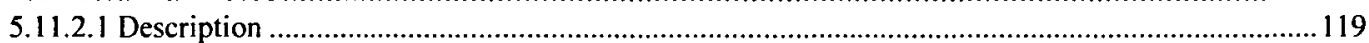

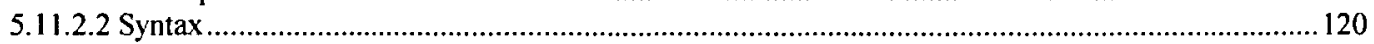

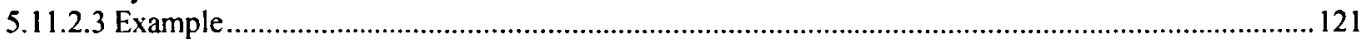

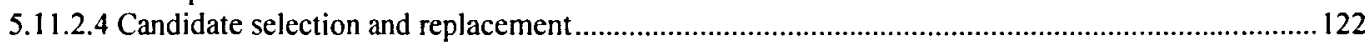

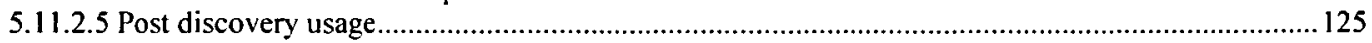

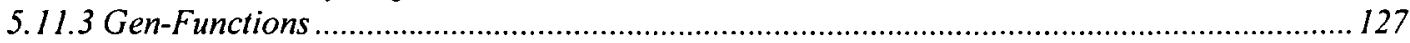

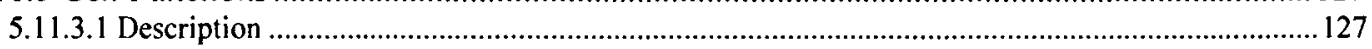




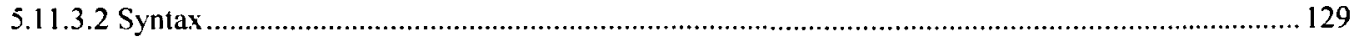

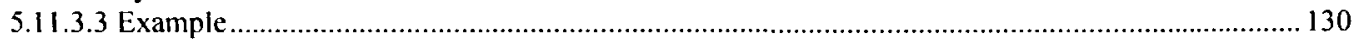

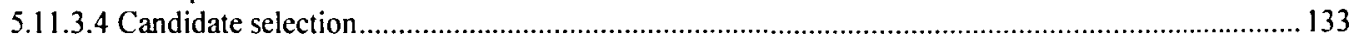

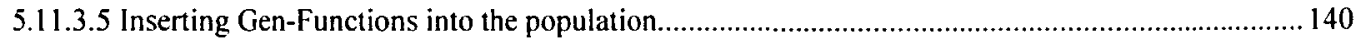

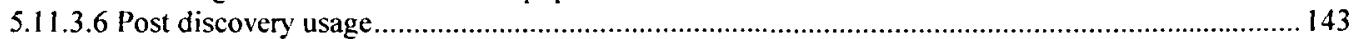

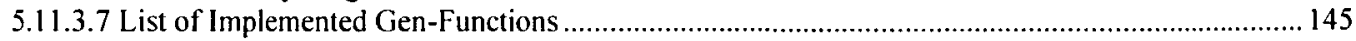

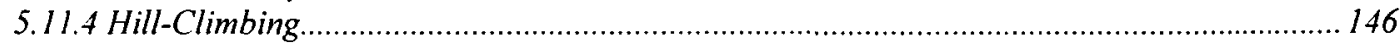

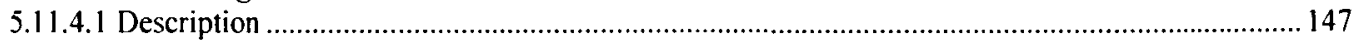

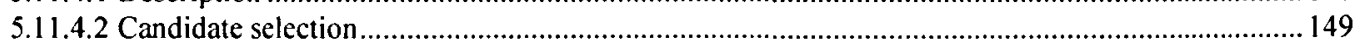

5.11.4.3 Hill Climbing as a Rule Mutation Operator ....................................................................... 151

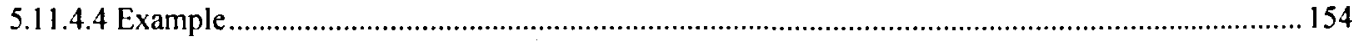

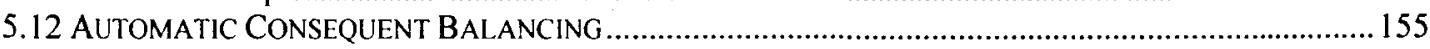

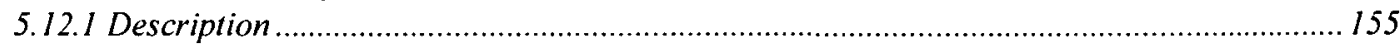

5.12 .2 Implementation details............................................................................................. 156

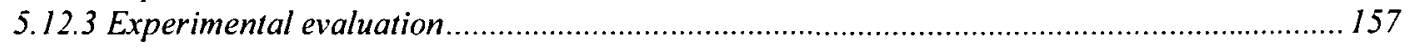

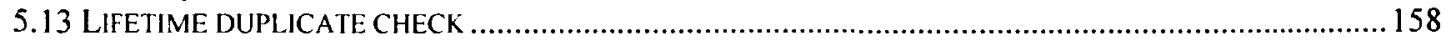

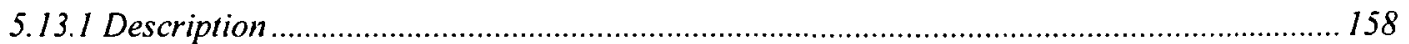

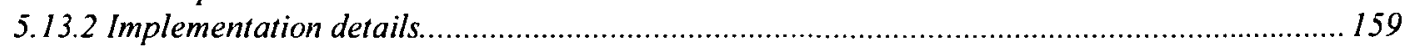

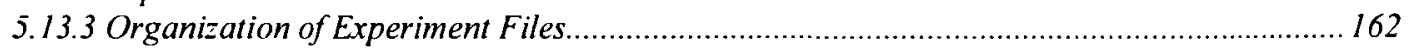

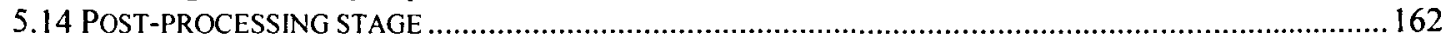

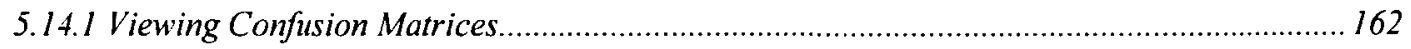

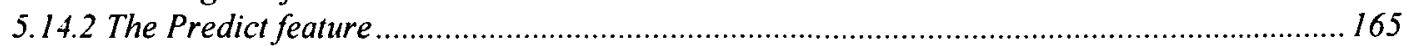

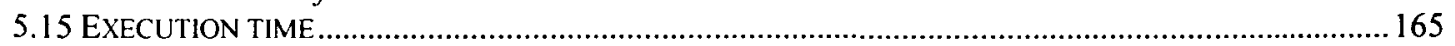

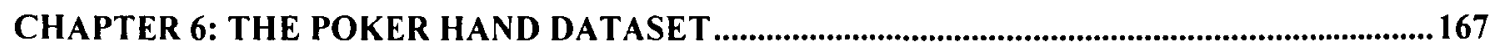

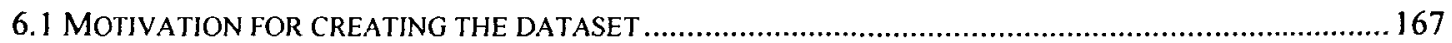

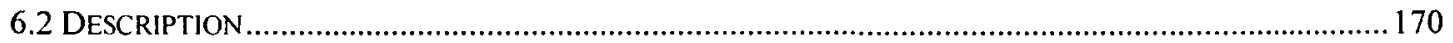

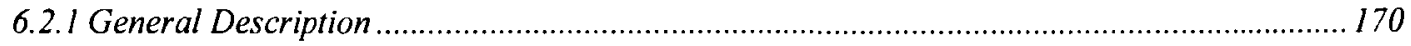

6.2 .2 Reasons that this is a difficult problem.................................................................... 172

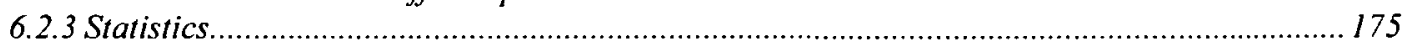

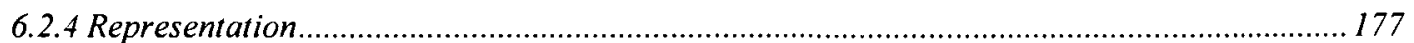

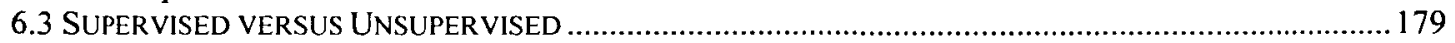

6.4 MAPPING TO REAL-WORLD PROBLEM DOMAINS ............................................................ 180

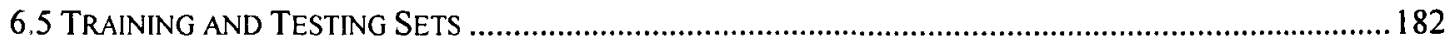

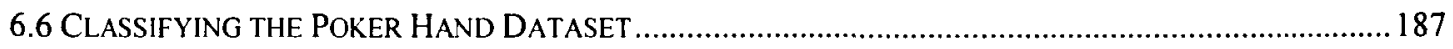

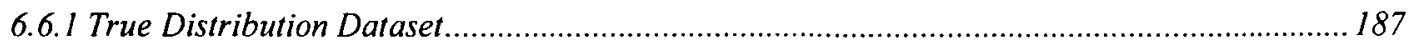

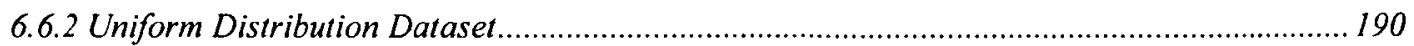

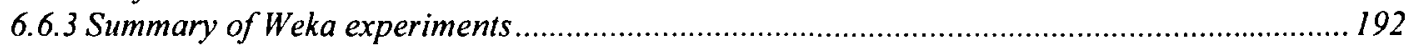

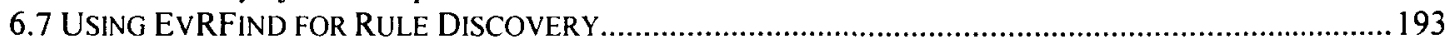

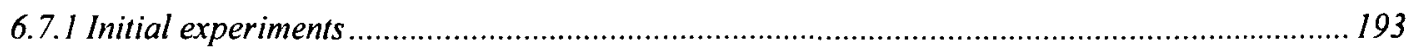

6.7.2 Experiments with EvRFind including Gen-Functions ............................................ 198

6.7.2.1 Overall Results - Uniform versus True Distribution .................................................................. 198

6.7.3 Evaluating the Hierarchy Protected Fitness option ............................................................200

6.7.4 Discovery of Concepts within the dataset..........................................................................201

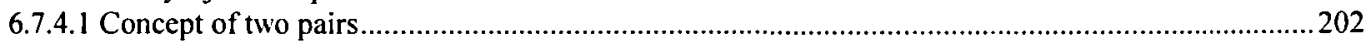

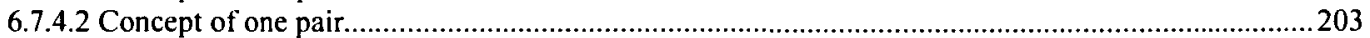

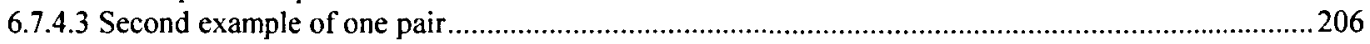

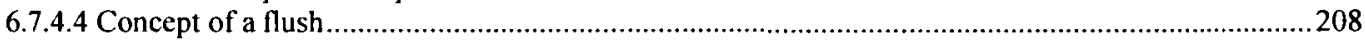

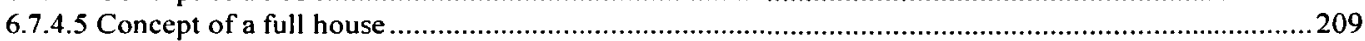

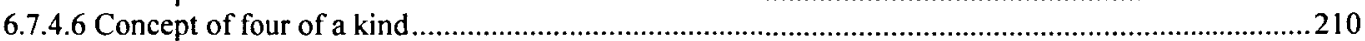

6.7.4.7 Concept of three of a kind

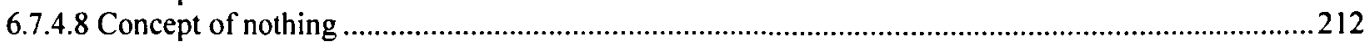

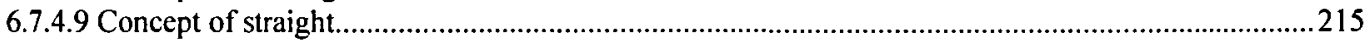

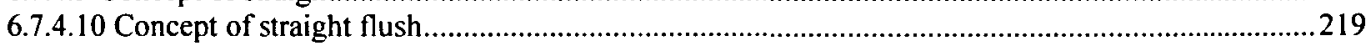

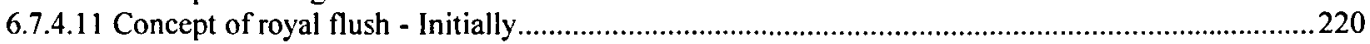

6.7.4.12 Concept of royal flush - Revisited .........................................................................................221

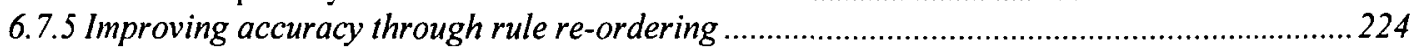

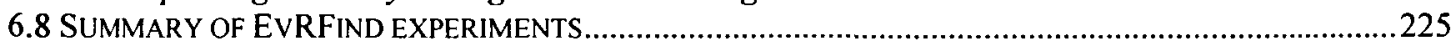




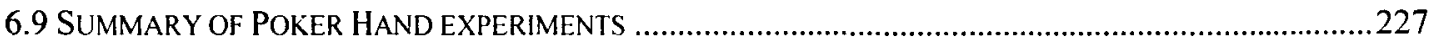

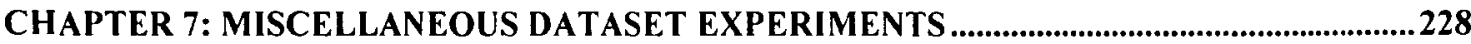

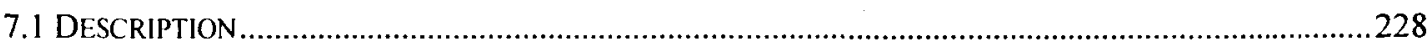

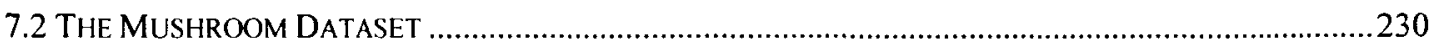

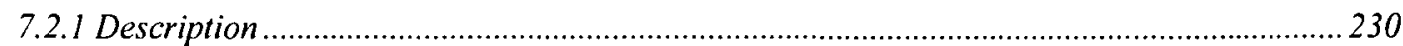

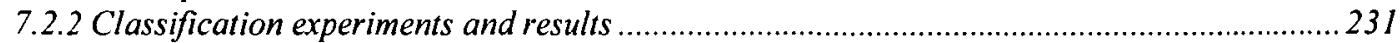

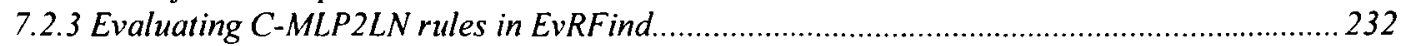

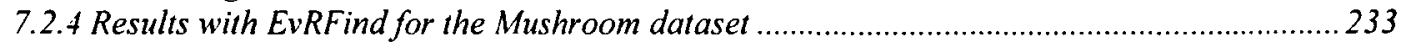

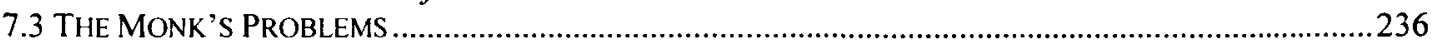

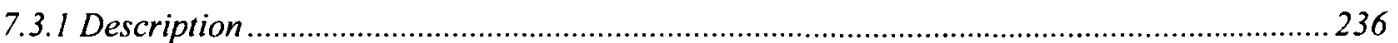

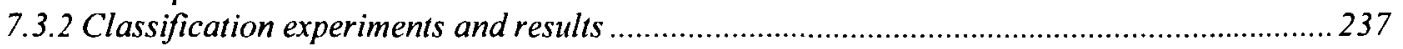

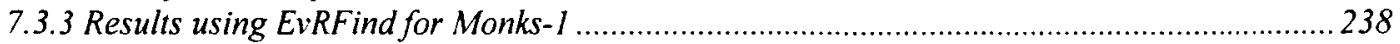

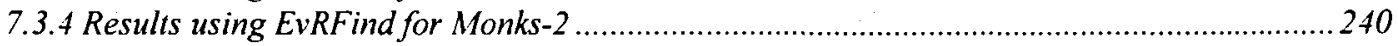

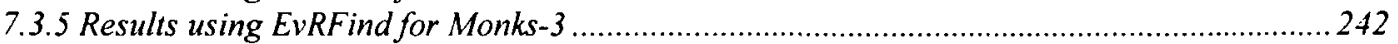

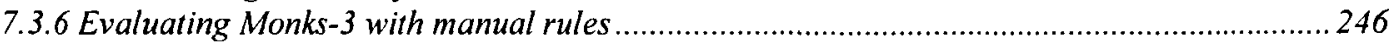

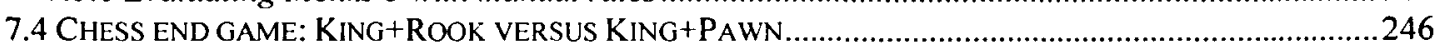

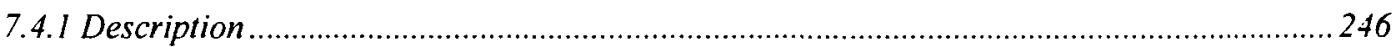

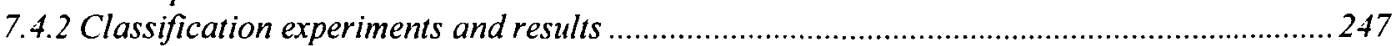

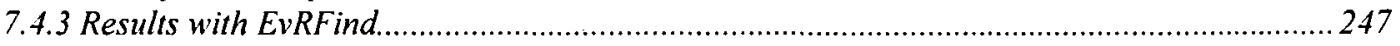

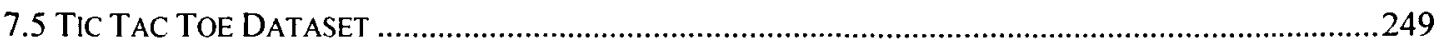

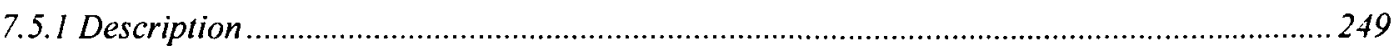

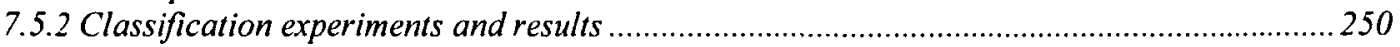

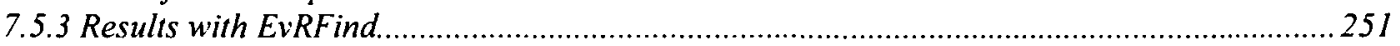

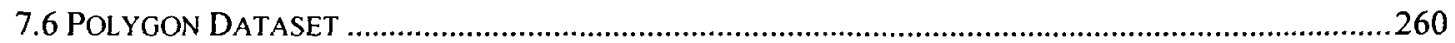

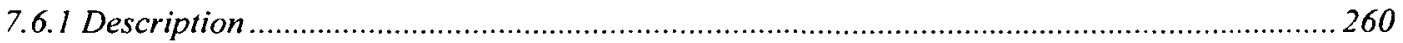

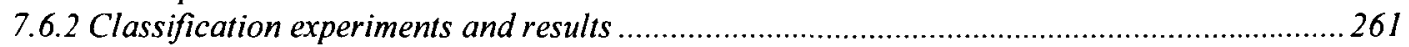

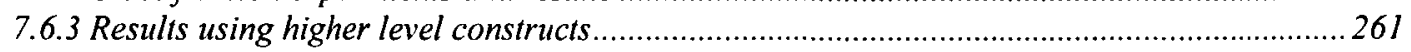

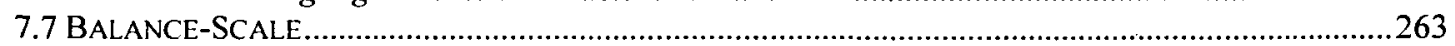

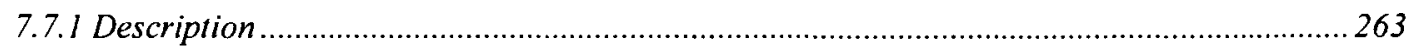

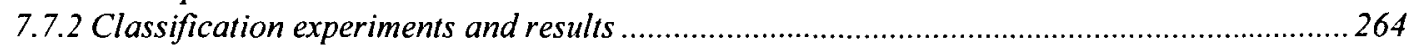

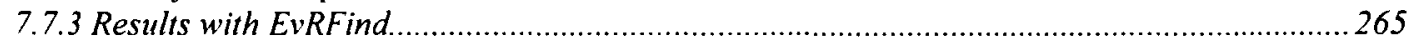

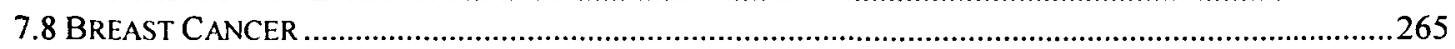

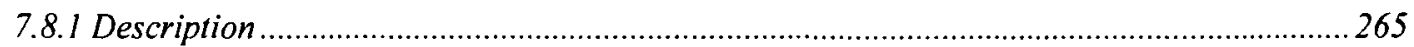

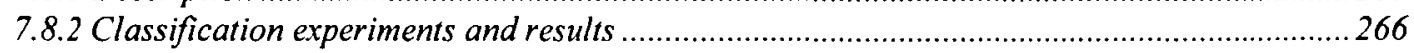

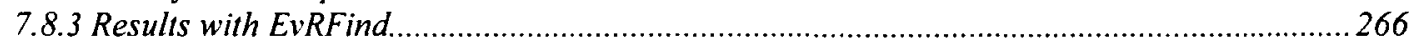

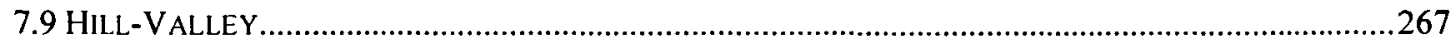

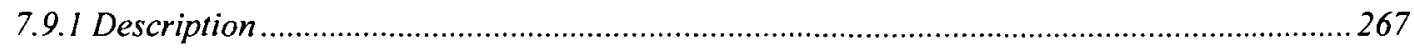

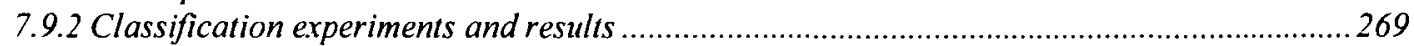

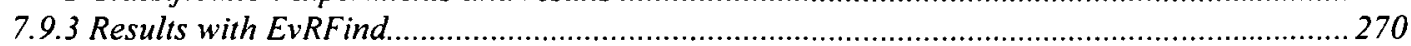

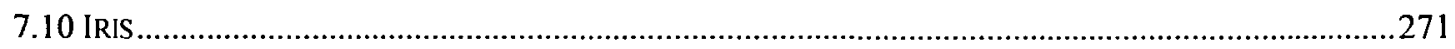

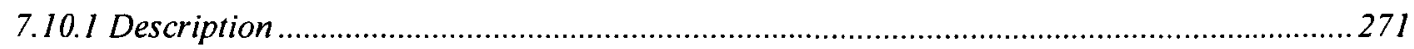

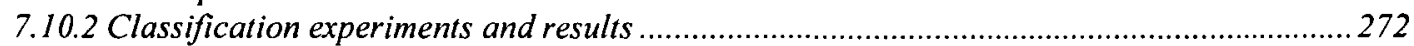

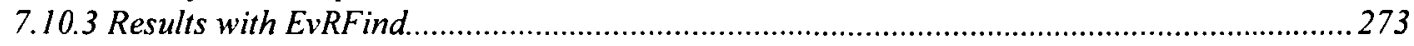

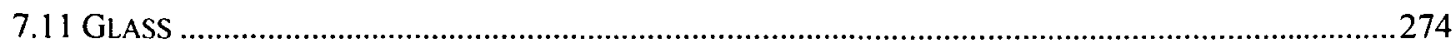

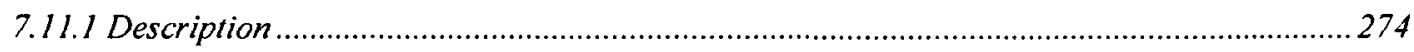

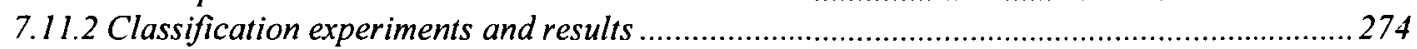

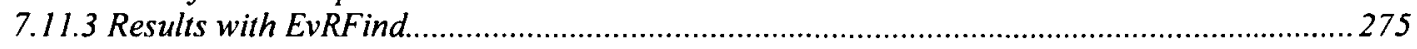

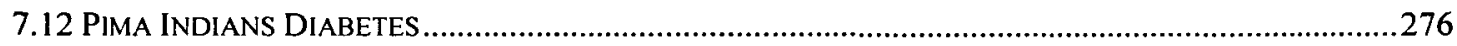

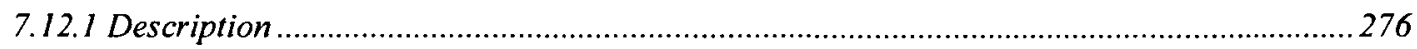

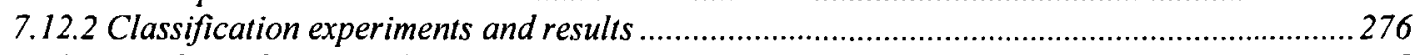

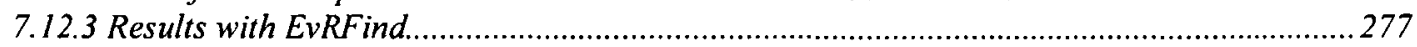

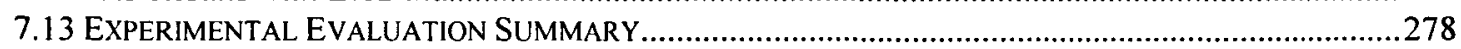

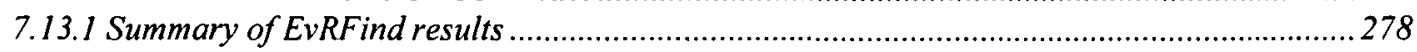

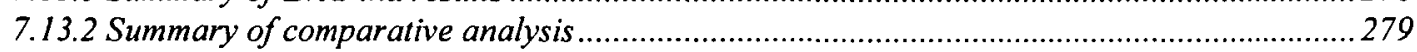

7.13.3 Summary of experiments with hierarchy-protected-fitness.................................................281 
7.13.4 Summary of experiments for the lifetime duplicate check.....................................................2. 283

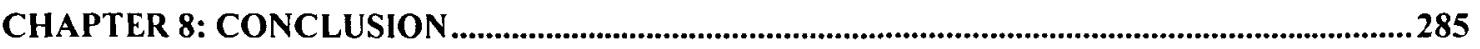

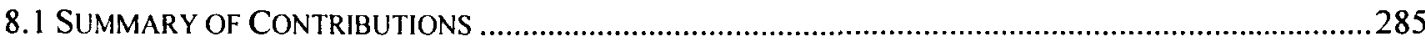

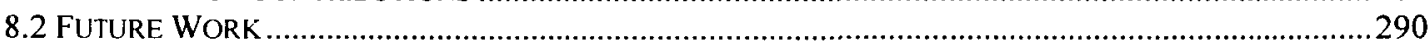

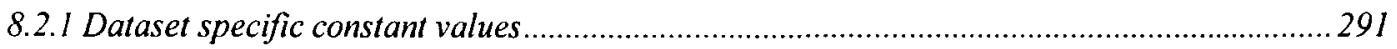

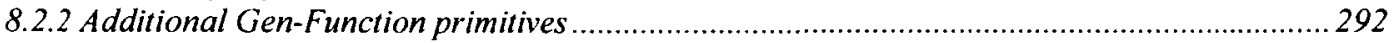

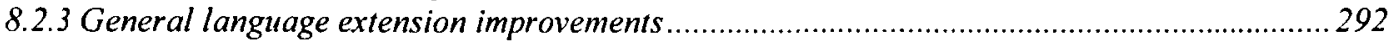

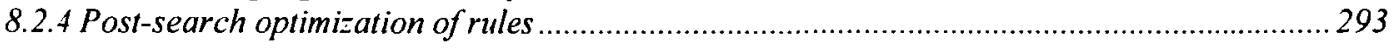

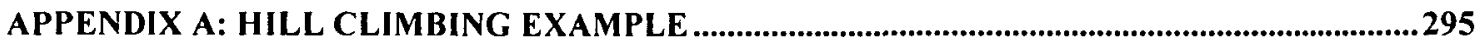

APPENDIX B: GEN-SET OPERATIONS AND PROPERTIES .......................................................297

APPENDIX C: DEFINITION AND SCORING OF POKER HANDS ................................................300

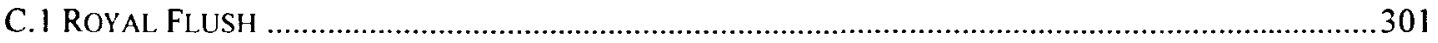

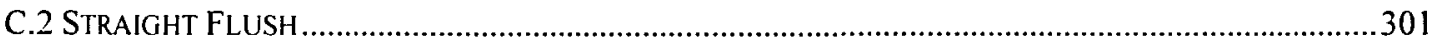

C.3 FOUR OF A KIND

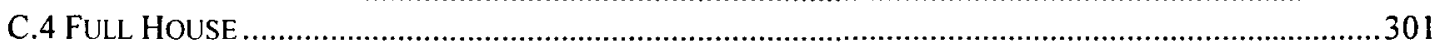

C.5 FLUSH .

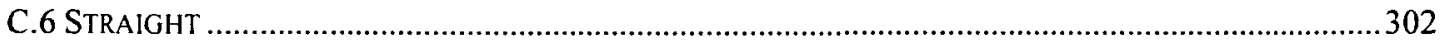

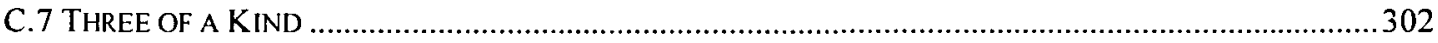

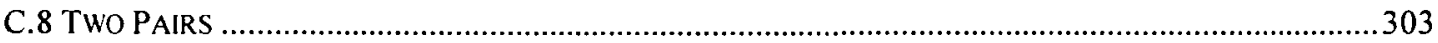

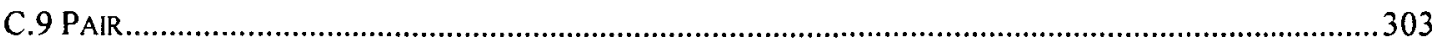

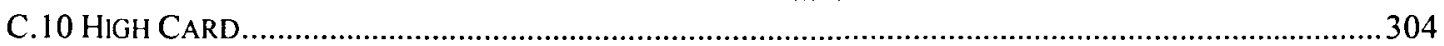

APPENDIX D: CALCULATION OF POKER HAND STATISTICS ................................................305

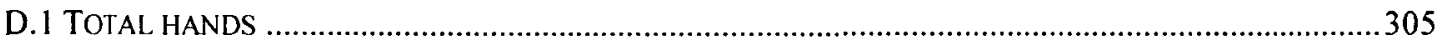

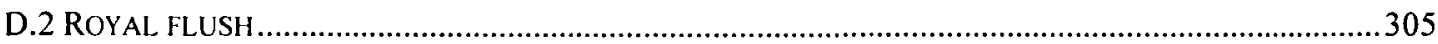

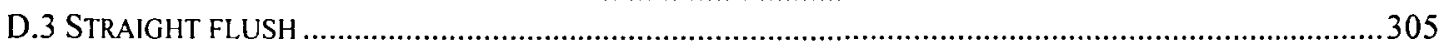

D.4 FOUR OF A KIND

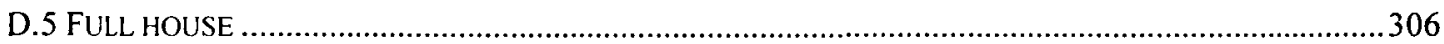

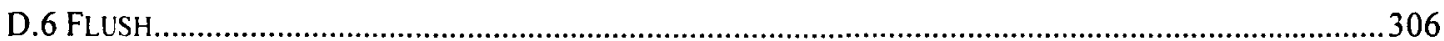

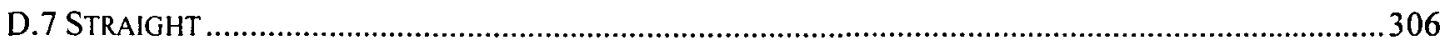

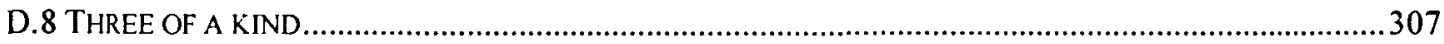

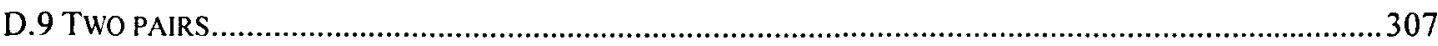

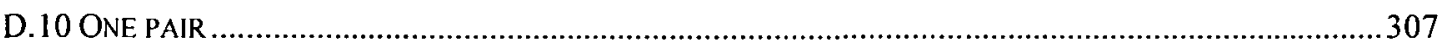

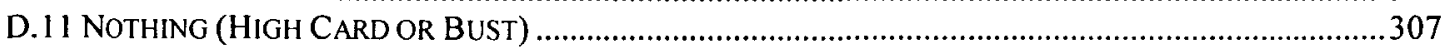

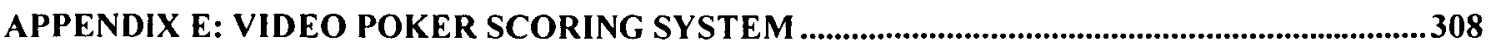

APPENDIX F: EXAMPLES OF MINIMAL RULE SETS .................................................................310

APPENDIX G: FUTURE GEN-FUNCTION PRIMITIVES ...........................................................3

APPENDIX H: DEFAULT PARAMETER SETTINGS ...............................................................

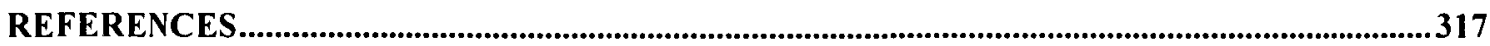




\section{List of Tables}

TABLE 5.1: EXAMPLE INSTANCES REFERENCED TO CREATE GEN-FUNCTIONS ......................................... 138

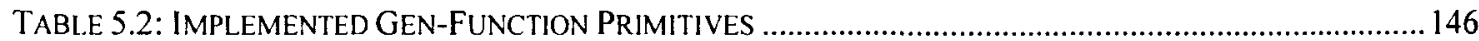

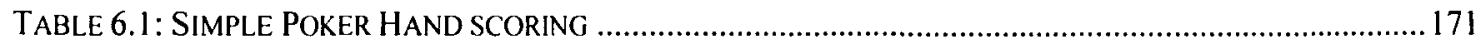

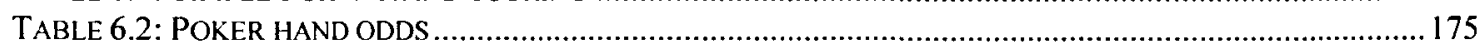

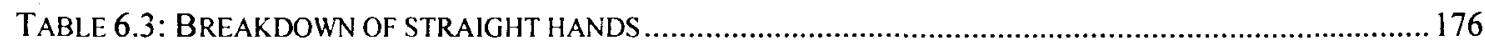

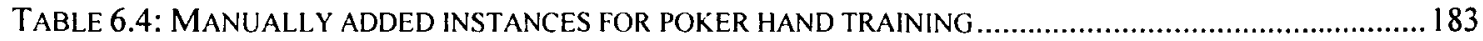

TABLE 6.5: STATISTICS FOR THE POKER HAND TRUE DISTRIBUTION TRAINING SET ..................................... 184

TABLE 6.6: STATISTICS FOR THE POKER HAND UNIFORM DISTRIBUTION TRAINING SET ............................... 185

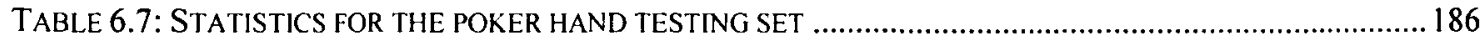

TABLE 6.8: ALGORITHM PERFORMANCE ON MODEL TRAINED WITH THE TRUE DISTRIBUTION.......................187

TABLE 6.9: ALGORITHM PERFORMANCE ON MODEL TRAINED WITH THE UNIFORM DISTRIBUTION................. 190

TABLE 6.10: INITIAL POKER HAND EXPERIMENTS WITH EVRFIND - UNIFORM DISTRIBUTION ........................ 194

TABLE 6.1 I: INITIAL POKER HAND EXPERIMENTS WITH EVRFIND - TRUE DISTRIBUTION ……......................194

TABLE 6.12: EXCERPT FROM EVRFIND HIERARCHY - INITIAL EXPERIMENTS............................................. 195

TABLE 6.13: POKER HAND EXPERIMENTS WITH GEN-FUNCTIONS - UNIFORM DISTRIBUTION........................ 198

TABLE 6.14: POKER HAND EXPERIMENTS WITH GEN-FUNCTIONS - TRUE DISTRIBUTION ............................ 199

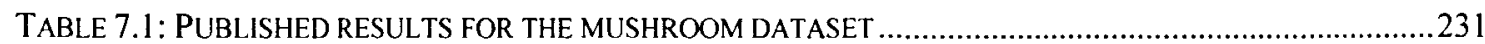

TABLE 7.2: RULES CREATED BY C-MLP2LN FOR THE MUSHROOM DATASET ............................................232

TABLE 7.3: RESULTS OF EVRFIND CLASSIFYING THE MUSHROOM DATASET ..............................................23

TABLE 7.4: COMPARISON OF POISONOUS RULES IN EVRFIND AND C-MLP2LN .........................................234

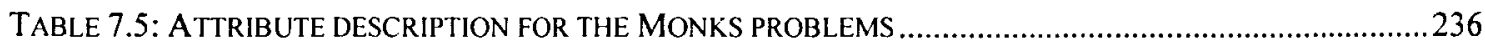

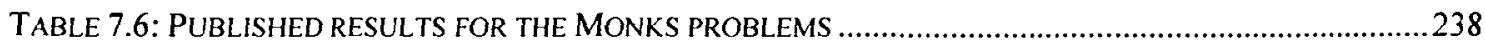

TABLE 7.7: MISCLASSIFIED MONKS-3 INSTANCES DURING ONE EVRFIND EXPERIMENT .............................245

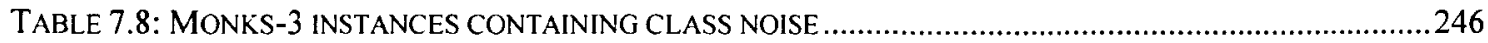

TABLE 7.9: PUBLISHED RESULTS FOR THE KING+ROOK VERSUS KING+PAWN DATASET ..............................247

TABLE 7.10: RESULTS OF KING+ROOK VERSUS KING+PAWN DATASET USING EVRFIND .............................248

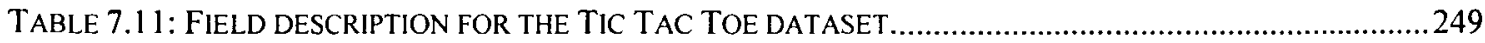

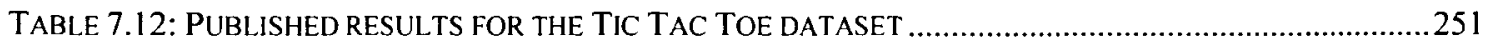

TABLE 7.13: INITIAL EVRFIND RESULTS FOR THE TIC TAC TOE DATASET .............................................2 251

TABLE 7.14: EVRFIND RESULTS USING LOW-SUPPORT FOR THE TIC TAC TOE DATASET ............................257

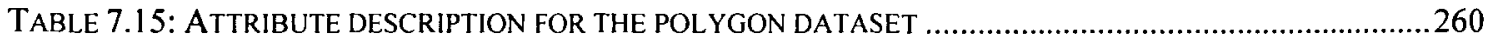

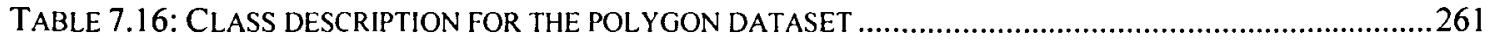

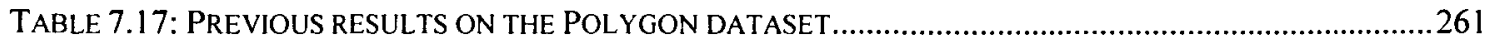

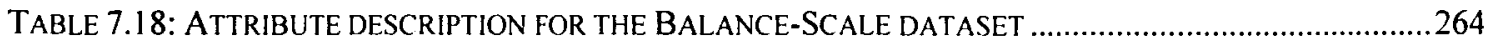

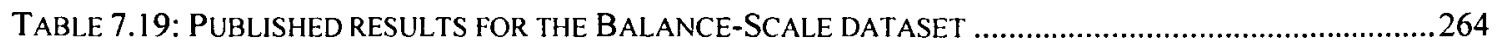

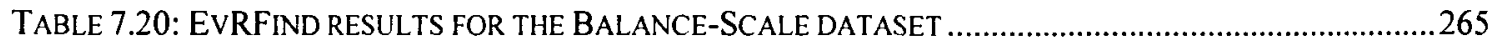

TABLE 7.21: PUBLISHED RESULTS FOR THE BREAST-CANCER DATASET ...................................................26

TABLE 7.22: EVRFIND RESULTS FOR THE BREAST-CANCER DATASET ……............................................26

TABLE 7.23: ALGORITHM PERFORMANCE ON THE HILL-VALLEY DATASETS.............................................270

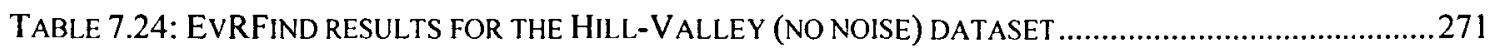

TABLE 7.25: EVRFIND RESULTS FOR THE HILL-VALLEY (WITH NOISE) DATASET …...................................271

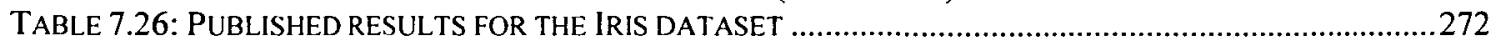

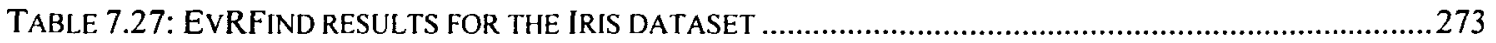

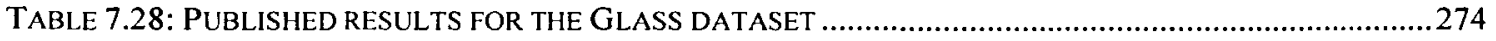

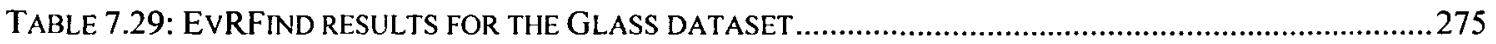

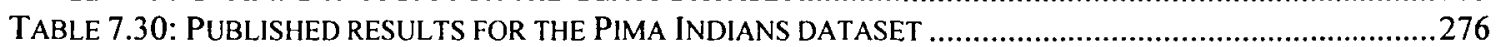

TABLE 7.31: EVRFIND RESULTS FOR THE PIMA INDIANS DIABETES DATASET ........................................277

TABLE 7.32: EVRFIND EVALUATION SUMMARY FOR POPULAR DATASETS ................................................278

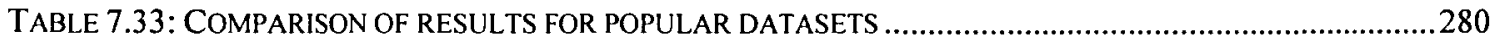

TABLE 7.34: SUMMARY OF MISCELLANEOUS HIERARCHY-PROTECTED-FITNESS EXPERIMENTS ......................282

TABLE 8.1: COMPARING VIDEO POKER AND SIMPLE POKER SCORING SYSTEMS ............................................308

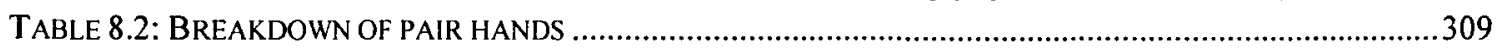




\section{List of Figures}

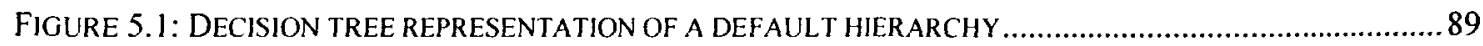

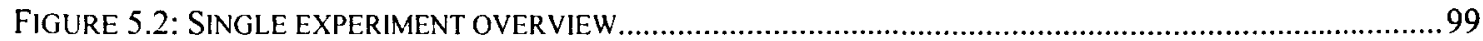

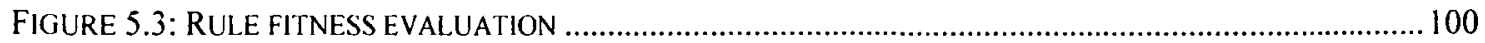

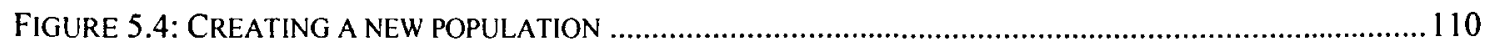

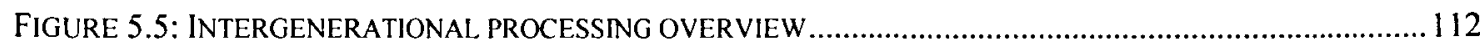

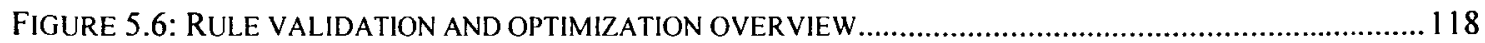

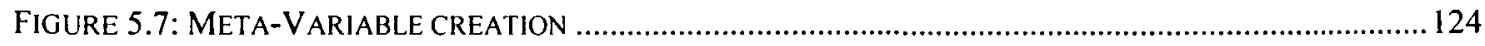

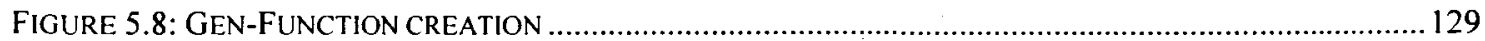

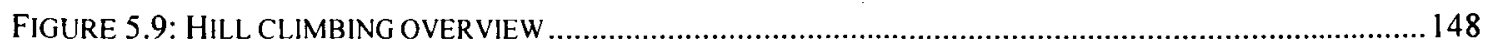

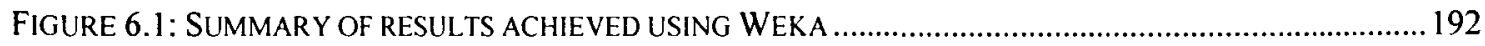

FIGURE 6.2: CLASS COVERAGE FOR THE JRIP ALGORITHM ON THE POKER HAND DATASET ......................... 193

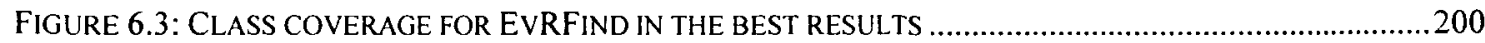

FIGURE 6.4: CLASS COVERAGE COMPARISON BETWEEN EVRFIND AND JRIP …......................................22

FIGURE 7.1: EXAMPLE OF 'VALLEY' INSTANCE FROM HILL-VALLEY DATASET WITHOUT NOISE ....................268

FIGURE 7.2: EXAMPLE OF 'HILL' INSTANCE FROM HILL-VALLEY DATASET WITH NOISE ..............................269

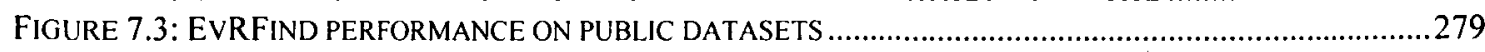

FIGURE 7.4: COMPARISON OF EVRFIND TO BEST COMPETING ALGORITHM ….........................................28 


\section{List of Appendices}

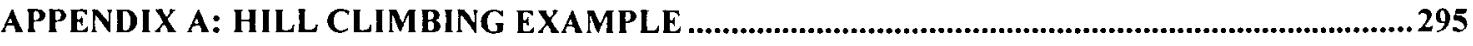

APPENDIX B: GEN-SET OPERATIONS AND PROPERTIES ............................................................297

APPENDIX C: DEFINITION AND SCORING OF POKER HANDS ..............................................300

APPENDIX D: CALCULATION OF POKER HAND STATISTICS ....................................................305

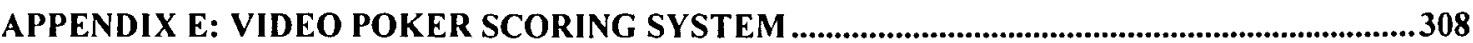

APPENDIX F: EXAMPLES OF MINIMAL RULE SETS ..............................................................

APPENDIX G: FUTURE GEN-FUNCTION PRIMITIVES ..................................................................313

APPENDIX H: DEFAULT PARAMETER SETTINGS ................................................................... 


\section{Chapter 1: Introduction and Outline}

This thesis studies the task of rule discovery within the larger problem of data mining. The platform used for this research is a new rule discovery system named EvRFind. This system makes extensive use of genetic algorithms and other machine learning techniques.

This chapter will briefly define these concepts, introduce EvRFind, describe the content and establish the order of reading for the remainder of this thesis.

\subsection{Data Mining and Rule Discovery}

Data Mining, also referred to as Knowledge Discovery in Databases, is a general term that refers to the automatic discovery of patterns within large volumes of data.

Data mining combines techniques including statistical analysis, visualization, decision tree induction, genetic algorithms, and neural networks to explore large amounts of data. The available tools are borrowed from several disciplines, such as statistics, pattern recognition and machine learning.

The main goal of a data mining system is to discover previously unknown facts about the data in a specific domain. The most common use for this is to build a predictive model, which can be used to predict the response for queries of interest. Although data mining is used for a variety of other tasks, such as intelligently breaking down large blocks of data into more meaningful categories, the focus of this work will be on predictive data mining. 
A common way to represent a predictive model is by using a set of If-Then rules that are made available by a rule discovery algorithm. A more complete description of data mining, predictive models, rule discovery and the related issues is given in Chapter 3 : Data Mining - Past and Present, starting on page 27.

\subsection{Genetic Algorithms}

A Genetic Algorithm (GA) [Holland, 1975] is one example of a heuristic search that is used to find solutions to optimization problems. In an optimization problem there are many known solutions, and the goal is to find the best one. The comparison of solutions is done using a measure that is often dependent on the problem domain. In some cases a solution will yield a more accurate answer, while in other cases a solution will represent a process that runs more efficiently than another.

GAs rely on heuristics to gauge the quality, or fitness, of solutions. These heuristics are normally approximations and not guaranteed to find the optimal solution to any given problem. Since the mid-nineteen eighties, however, GAs have been used with success in a variety of applications. Some of these include biology, engineering, pattern recognition, the physical sciences and the social sciences [Goldberg, 1989].

The strength of a GA is its ability to search large finite spaces by evolving complex solutions. Solutions are evolved through the application of genetic operations, and solutions are modified and combined in an environment where they are forced to compete 
with each other. The entire process is inspired by Darwinian natural selection and the field of genetics.

A more complete description of the GA, including usage and implementation details, is given in Chapter 2: Introduction to Genetic Algorithms, starting on page 22.

\subsection{EvRFind}

At the core of this research is EvRFind - "Evolutionary Rule Finder". This system relies heavily upon GAs and other methods for the task rule discovery. The implementation, testing, and experimental evaluation of EvRFind are the main subjects of this work, and a detailed description can be found starting on page 67, in Chapter 5: EvRFind - Detailed Description.

\subsection{Objectives}

\subsubsection{Primary goals and scope}

The main goal of this research is to develop methods to increase the potential for rule discovery in large or complex databases. Further to this, an emphasis will be placed on both rule accuracy and comprehensibility, with a strong bias towards discovering rules that are of minimal length.

These goals will be achieved by developing an application to extend the functionality of the standard GA such that it is suitable in a data mining environment. By combining both 
evolutionary and non-evolutionary techniques, as well as methods from other areas of machine learning, the new system will be more effective in some domains than other methods that are currently being used.

\subsubsection{Experimental evaluation}

Evaluating the performance of this application on a variety of tasks is an important part of the research, and this is achieved using two different methods. First, a new synthetic dataset is fully described and then analyzed with several well known machine learning algorithms. The results of these experiments are compared to those produced by EvRFind. As will be seen in Chapter 6.9, Summary of Poker Hand experiments, starting on page 227 , EvRFind does significantly better than the other algorithms that are evaluated.

The second experimental phase involves using EvRFind for the discovery of rules in several publicly available datasets. The results of these experiments are compared to the results of several different methods that are found in other published research. In the summary of this work, located in Chapter 7.13, Experimental Evaluation Summary, starting on page 278, it can be seen that the performance of EvRFind varies largely depending on the dataset. On some datasets, however, the results are significantly better than those discovered by algorithms found in other published research. 


\subsubsection{Contributions}

The research in this dissertation goes beyond what is currently being done in the fields of genetic algorithms and data mining. This work includes the contribution of new techniques to the field of GAs and evolutionary computing. Although some of these features are tailored to the task of rule discovery, they are general enough to be used in a generic application of GA. As the domain of choice for the improved GA, the field of data mining will also benefit from these features. Furthermore, the contribution of the Poker Hand dataset will aid in the evaluation of more advanced algorithms in the field of data mining.

\subsubsection{Summary of contributions and intermediate objectives}

The main contributions of this research can be summarized as follows:

- The evolution of default hierarchies

- The implementation of "on the fly" constructive induction, where it is performed while the search is in progress

- The combination of methods from different areas of machine learning such that the system still takes advantage of the evolutionary power offered by the GA

- The automatic generalization of concepts

- Automatic expansion of the grammar available to the GA

- The creation of a synthetic dataset to represent a problem domain that is difficult for existing classification and rule discovery algorithms 
The contributions in this research have been achieved by successfully completing a number of intermediate objectives. These objectives, given below, are more completely described in the corresponding sections of this thesis.

In summary, the milestone objectives described in this research are:

- Development of a Lifetime Duplicate Check that efficiently maintains a history of all solutions generated during a single experiment. This novel feature helps EvRFind avoid the problem of solution re-discovery by comparing new solutions with the history.

- Compile a list of public datasets and use them to evaluate EvRFind.

- Compare the results of this experimental evaluation against other published work. These results will show how EvRFind performs in comparison to other learning algorithms. This evaluation process will show that EvRFind performs equally or better than other learning algorithms in certain domains. This analysis will also highlight the areas where EvRFind has difficulty. This will be apparent in cases where the rules that are discovered produce results that are of lesser quality when compared to other systems.

- Experiment with the synthetic "Poker Hand" dataset using publicly available learning algorithms. These results will be used, in part, to show how difficult it is for a learning algorithm to accurately classify unseen items within the domain.

- Experiment with the synthetic dataset using the new system, EvRFind, and compare results to those discovered using the other publicly available learning 
algorithms. These results will show that EvRFind is superior at rule discovery in this domain.

\subsection{Thesis Outline}

This thesis addresses a number of issues that are relevant to the goal of meeting the objectives outlined in the previous section. Following is a brief description of each chapter, including details on what the key points are with respect to this research.

\section{Chapter 2: Introduction to Genetic Algorithms}

Chapter 2 provides an introduction and overview to Genetic Algorithms. The terminology and methods are described for the traditional GA implementation. This includes descriptions of the fitness function, how the data is represented, and the various genetic operations that are used to manipulate rules within the population. Hybrid algorithms and approaches are also described. This chapter is intended to give the reader a basic understanding of the GA and how it relates to this research.

\section{Chapter 3: Data Mining - Past and Present}

Chapter 3 introduces data mining and provides details on important concepts such as predictive models, confidence and support factors, and the difference between supervised and unsupervised learning. Details are given for some of the more common issues that create problems for data mining applications, and references within this thesis are made for issues that are later discussed. A summary of existing data mining techniques is 
provided, as well as information on areas that are not adequately addressed by modern day rule discovery systems.

\section{Chapter 4: Literature Review}

Chapter 4 is a review of existing literature that covers the related topics in this thesis, with the focus on evolutionary rule discovery and constructive induction.

\section{Chapter 5: EvRFind - Detailed Description}

Chapter 5 presents the development of EvRFind, a hybrid genetic algorithm based system used for the task of rule discovery in data mining applications. Included is a description of how EvRFind represents solutions internally, and the rationale for using it. A default hierarchy is described in the context of its use within the system. Details of features such as learning from experience, automatic generalization, non-evolutionary search, and building on the existing grammar are described. These descriptions include the purpose of design, implementation details, and some experimental results.

\section{Chapter 6: The Poker Hand Dataset}

Chapter 6 introduces the topic of synthetic datasets, and why they are sometimes used to evaluate data mining algorithms and applications. A new dataset, the Poker Hand Dataset, is fully described. This includes the motivating factors for its creation and use, including what makes it different from other synthetic datasets, as well examples of how to map it onto other real-world problems. The results and analysis of extensive 
experimental evaluation using EvRFind, and other machine learning algorithms, are shown.

\section{Chapter 7: Miscellaneous Dataset Experiments}

Chapter 7 describes several publicly available datasets. These datasets are a combination of synthetic and real data, and include several that are well-known for evaluating data mining systems. EvRFind is used for rule discovery on each one, and the results are presented with an analysis and comparison to other published results generated by a variety of machine learning algorithms.

\section{Chapter 8: Conclusion}

Chapter 8 summarizes the research in this thesis by restating the objectives and outlining the points related to each goal. This chapter concludes with some discussion on what direction this research is headed in, and includes suggestions of features to be included in future implementations. 


\section{Chapter 2: Introduction to Genetic Algorithms}

\subsection{Description}

Genetic Algorithms were introduced by Holland and first published in Adaptation in Natural and Artificial Systems [Holland, 1975]. GAs are part of the larger area known as evolutionary computing, in which solutions to problems are evolved using techniques inspired by evolutionary biology.

GAs operate by evolving populations of individual solutions from one generation to the next. The evolution is a multi-step process, starting with an initial population that is created using a randomly generated set of members. As individuals interact with the environment they are subject to tests and changes that dictate whether they are discarded, or continue into the next generation.

The environment acts as an interface between the members of the population and the problem that is being solved. As such, the primary role of the environment is to assess the worth of each potential solution. This is done using a routine called the fitness function.

\subsection{Traditional GA}

\subsubsection{Fitness Function}

The fitness function calculates a score, or measure, indicating the distance between a solution and the goal state. These functions are problem dependant and typically rely on a 
set of heuristics to approximate the distance to the goal, and then assign an appropriate score. This score is referred to as the fitness of the individual.

Fitness calculation and the related heuristics are an important area of study for any given problem. The concept of a perfect heuristic is typically not possible because it would require the ability to solve a problem that is not solvable by means other than exhaustive search. The practical answer is to use heuristics that provide an approximation of how close the goal state is.

It is common to have different heuristics for the same problem domain. In most cases, the more accurate heuristics come at the price of an increased computational effort. Depending on how much longer it takes to execute a higher quality fitness function, it may even be more efficient to use an inferior estimator because more states can be searched in the same amount of time.

\subsubsection{Representation}

In order to solve a problem using a GA, it is necessary to find an internal representation that is compatible with the fitness function and other genetic operations. The standard representation for each solution is a fixed length, binary encoding [Holland, 1975; Goldberg, 1989]. With each individual being represented by a bit string, it makes it easy to perform many of the genetic operations that are commonly used in GA implementations. 


\subsubsection{Selection}

Selecting an individual refers to the process where a solution is chosen and allowed to continue in the next generation. Although, for the most part, this operation is probabilistic in nature, there is a bias towards selecting individuals with higher fitness. This process mimics the Darwinian concept of survival of the fittest, where even a slight genetic advantage corresponds to an increased chance of survival. Continued survival translates to a higher rate of reproduction, which in turn increases the likelihood that an individual's genetic makeup will persist in the population.

There are a large number of variations in use by GA systems, but the most popular is fitness proportional selection. EvRFind uses a modified version of this selection method and it will be described in more detail in the corresponding chapter.

\subsubsection{Crossover}

After an individual is selected from the population there is a probabilistic opportunity that it will be subject to a recombination step, named crossover. Crossover involves selecting a second member from the same population, also with a bias towards a higher fitness. The two individuals selected are referred to as the "parents".

After the parents are selected there is a representation-dependent method used for combining them. The result is normally two new individuals that are both different combinations of the originals. These are referred to as the "children". 
Crossover is used in the hope that good sections from each of the two chromosomes will be combined to make at least one child that is superior to the parents. This is possible because two individuals with a higher-than-average fitness can be good for different reasons, and when recombined it makes for an even better aggregate fitness. In the traditional GA the parents are discarded and the children are moved into the next generation.

The effectiveness, as well as the potential disruption caused by the crossover operation, is dependent on several factors including the representation, choice of crossover method, and the size of the population. This is described in [DeJong, Spears, 1991].

\subsubsection{Mutation}

Mutation is a genetic operator that is used to probabilistically modify solutions at the bit level. In a standard GA there is a random probability of mutation for every bit in the chromosome. This means that some individuals will not be mutated at all, while others might have a single bit mutation, and it is possible that others are subject to multiple mutations.

The purpose of mutation in GAs is to maintain genetic diversity and prevent the population from prematurely converging on a single solution. This helps to prevent the trap of a local optimum value, where the population becomes stuck and is unable to find better solutions. 


\subsection{Hybrid approaches}

A hybrid algorithm solves problems using a combination of more than one method. The intention is that the good properties of each method will be used in conjunction with each other to solve the problem more efficiently than either would in isolation. Also worth noting is that many good results are possible using the techniques described here, but not all methods are compatible in every environment. Hybrid approaches can recognize what techniques are best suited to the current task or domain.

Hybrid algorithms are created when one technique works well on a subset of the problem, but performs below average on another part. For example, a purely evolutionary system using a GA is not always ideal because GAs are good at finding the general area of the solution, but not as well suited to fine tuning [Zhang, Ishikawa, 2004; Prügel-Bennett, 2004]. A Memetic Algorithm [Moscato, 1989] is an example of one that combines GAs with local search techniques to achieve the best results. Memetic algorithms are also referred to as Hybrid Genetic Algorithms (HGAs).

HGAs have been applied to a variety of problems in different fields. Typically, the implementation varies in terms of how the fine-tuning search is performed. A survey on HGAs is available in [Sinha, Goldberg, 2003].

With respect to this research, EvRFind is an example of a genetic algorithm hybrid. This is more completely described starting on page 67, in Chapter 5: EvRFind - Detailed Description. 


\section{Chapter 3: Data Mining - Past and Present}

\subsection{Building predictive models}

There are many techniques used to extract knowledge from data and then infer a predictive model. A predictive model, at least in the case of supervised learning, relies on attributes within the database to predict a value or combination of values that define a class. The attributes relied upon are referred to as predictive attributes.

Depending on the methods used during the discovery process the model will assume a particular form. For example, a visualization technique might produce charts and graphs, while a rule induction algorithm will produce sets of if-then rules. For classification tasks, where a set of pre-defined classes for the data exist, if-then rules are referred to as classification rules.

\subsubsection{Classification Rules versus Association Rules}

A predictive model that is comprised of an accurate and complete set of classification rules is able to classify data, based only on the predictive attributes, and correctly label it as one of the pre-defined classes. One of the problems with the automatic discovery of classification rules is finding a way to determine how useful a particular rule is going to be. This has also been a topic of research in the more specific problem of association rule discovery. 
Association rules are similar in form to the classification rules that are described above, but their use is more specific. An association rule learning algorithm is used for discovering regularities between products in large scale transaction data recorded by point-of-sale systems in a retail environment. This application of rule discovery is more commonly known as market basket analysis [Berry, Linoff, 1997].

Two of the widely accepted measures used for assessing the value of association rules are the confidence factor and support factor. These are important concepts in this research because the EvRFind system implements these measures, and takes their estimations of worth into account in the determination of value for the more general classification rules.

\subsection{Confidence and Support Factors}

The quality of a classification rule is based on several factors such as accuracy, coverage, how useful and how interesting the rule is within its domain. There is no widely accepted measure for how interesting a rule is because the entire concept is subjective in nature. This creates a difficulty because without input from the user, the automatic assessment of quality must rely on statistical methods for accuracy and coverage.

As mentioned in the previous section, EvRFind uses the same measure for accuracy that is calculated when evaluating the worth of an association rule. The confidence factor reflects how often the consequent is true given that the antecedent is true. If the consequent is false when there is a match with the antecedent, then the confidence fails 
for the given rule. If the antecedent is not matched by the example instance then the instance does not contribute to the determination of the confidence of the rule.

Also in common with the evaluation of association rules is the support factor, which is a measure of the rule coverage. This refers to how often the rule is correctly applied within the entire dataset. The measure is calculated by dividing the number of elements that are correctly classified, using the rule being scored, by the total number of example instances in the dataset. It essentially indicates how often the rule is actually used, which is an important factor to consider when determining its worth.

There are no perfect values to use when setting confidence and support factors, but there are a few recommended guidelines that should be considered. When the goal is a predictive model for classification it is often best to maximize both confidence and support. The best rules are $100 \%$ accurate, and are normally general enough to cover as many example instances as possible.

When the goal of the search is unsupervised, such as in the case of a market basket analysis, setting the confidence and support goals too high will often cause the search to be ineffective. The reason for this is that the rules, or associations, being sought are not obvious. In these types of searches it is normally the case where knowledge discovered with high confidence and support factors is already known, such as in the case of an axiom or another type of domain specific knowledge. While this might be useful for classification tasks, it contributes very little towards the goal of knowledge discovery. 


\subsection{Directed versus Undirected Data Mining}

During the knowledge discovery process there are points where the user can supply the system with information. This information is gathered externally and is not related to the current process. The amount of information supplied corresponds to the degree in which the search is directed.

Directed data mining is also known as supervised learning. A common example of a supervised learner is a classification system, where the process attempts to discover concept descriptions. The data used for discovery is typically a set of pre-classified examples that are supplied by a teacher. The learning algorithm is able to adjust the predictive model by evaluating successive examples against it, and then making changes as necessary. The main problem with supervised learning is that it relies on human experts to create the classes, as well as update them periodically to reflect changes in trend.

Undirected data mining, also known as unsupervised learning, refers to the discovery of knowledge with little or no information supplied by the user. The goal is simply to find something interesting about the data, and do so without information provided by an expert. To illustrate the difference between these two approaches, consider a system that rates the quality of coffee beans.

In either type of search the dataset contains information such as the origin, physical description, chemical breakdown, and other objective information for example instances 
of coffee beans. Unique to the directed classification system is the presence of an additional field, which is a rating of quality by a human expert. In a binary classification system the beans might be labeled good or bad, but it is more likely that there are several degrees of quality with some kind of numerical rating.

After examining the attributes and class for each instance in the dataset, a supervised system will create a predictive model that will attempt to determine quality using the basic information only. This model can afterwards be used to classify unseen examples of coffee beans in the same fashion, but in the absence of the human expert.

In an undirected search the class attribute is not present, and the process will create groups based on the basic information only. In domains where a human expert has already determined the appropriate classes, one or more of the automatically created classes may correlate with them, although this is not always the case. The model might contain fewer groups than classes because of ambiguity in the data or large overlaps in classes. Also possible is that more groups than classes will emerge, where the set of groups is actually a superset of the classes.

The reason for extra groups being created is that more than one type of classification will occur concurrently. For example, groups can still be formed to describe quality, but other groups may describe attributes such as bean type or family. One of the difficulties involved with undirected data mining is the analysis of the results, where meaningful 
labels have to be given to each of the groups. Because the system has insufficient information for this task it is left up to the researcher in the post-discovery stage.

If during the post-discovery phase it is determined that the undirected search created more groups than is necessary, then the groups can be refined, joined, or separated depending on what granularity is required for the set of classes.

The main disadvantage with undirected data mining is that the process is more demanding than supervised learning, and as a consequence it takes considerably longer to perform. The tasks range from detecting potentially useful regularities among the data couched in the provided description language to the discovery of concepts through conceptual clustering and constructive induction, and to the further discovery of empirical laws relating concepts constructed by the system. The search is generally less focused and in many cases the analyst will not know what results to expect. Because of the variety in tasks and extra time required for both configuration and the search itself, undirected data mining is not possible for all projects.

Despite the penalty in time, undirected searches are becoming more widely used for two main reasons. The first is that some domains lack expert advice and opinions, and an appropriate classification scheme simply does not exist. In some cases there are clues as to what classes should exist, but in other cases there is no opinion as to what characteristics should be segmented into groups. The second reason is to try and break away from the bias created when training with a single human expert. As expert systems 
learn from example, they are credited by mimicking the teacher as closely as possible. Because of the possible difference in opinions between experts, it is not always beneficial to use a single teacher for extended periods of time.

\subsection{Issues in Data Mining}

A large number of the problems in data mining stem from the volume and type of data that these systems rely on as input. In most cases the data comes from a database that is not designed for the purpose of data mining. The raw data is often in a format that is unprocessed and not suitable for searching. Specifically, the attributes required to facilitate knowledge discovery are not present, and cannot be supplied by a third party.

Without certain attributes or information it might be impossible to discover significant knowledge in a particular domain. A trivial example of this is trying to diagnose diabetes without having an attribute describing the patient's blood sugar content. Without this indicator it may be impossible to diagnose the condition with $100 \%$ confidence.

In cases, however, where there is enough information to make useful discoveries, there are still other problems that inhibit the search process. Several of these issues, and how they relate to EvRFind, are discussed below. Not all of the issues are explicitly addressed by this research, but they are still briefly described in order to provide a broad understanding of the difficulties that can arise. 


\subsubsection{Noise and Missing Values}

With the possibility that a database contains erroneous or missing values, it cannot be assumed that the raw data is complete and correct. Examples of noise in the raw data are data entry errors, hardware interface problems, and the miscommunication of standards such as Celsius versus Fahrenheit. Missing values occur for many of the same reasons and present the same kinds of problems. Obviously it is important to minimize noise and missing values as these negatively impact the overall accuracy of the predictive model.

There are several strategies available for dealing with missing values, including ignoring them, omitting the entire record, using known values to infer a missing value, and treating missing values as a special symbol [Berry, Linoff, 1997]. Each of these, as well as other strategies, have advantages and disadvantages that become more or less apparent depending on the dataset and the domain.

The detection and correction of noisy values is sometimes possible depending on the distribution of values. In many cases the data will fit a regular statistical distribution, which means that the erroneous values will be identified. Unfortunately, the easiest ones to detect are extreme values that are often filtered out during the data entry process. It may be impossible to detect errors that appear within the proper range, although they may be revealed as anomalies during the building of a predictive model. In the worst cases, the model will incorporate the noisy values and the overall accuracy will be affected. The problems of missing and erroneous values are both handled indirectly by EvRFind. In the sections on implementation and experimental evaluation, these topics and their 
solutions will be described in more detail. Essentially, missing values are handled by the fact that rules created by EvRFind do not have to contain all of the attributes in the dataset. With respect to noisy data, EvRFind simply assumes that all of the data that is entered is valid. This approach may seem overly simple, but it is reasonable because in most cases there is no definite way to detect and correct errors. Interestingly, in many cases the rule discovery process eventually highlights noisy data because it appears to be special. An example of this is shown when training on the Monks-3 dataset, which is more completely described in Chapter 7.3, The Monk's Problems, starting on page 236.

\subsubsection{Merging Data from Multiple Sources}

Data integrity is an important consideration for data mining systems. One of the challenges is integrating data from different sources, where the data might contain redundant or conflicting values. In addition, data from other sources might be formatted differently or use different standards for the representation of values such as measurement. [Berry, Linoff, 1997].

During the integration process the data must be properly translated and verified according to the rules of the primary database. This important step can also be expanded to search for missing or noisy values, with the limitations that were described earlier. For auditing purposes the main database also keeps track of the original source. If problems appear to be more frequent from a particular source then it can be more heavily scrutinized or omitted from the merge. 
Also to consider is that during any merge process, there may be one or more irrelevant attributes. Although it is possible to incorporate this information into the rules used to perform the merge, this relies on a human expert to determine what is relevant versus what is not. In some cases, depending on how much is known about the domain, it is better to keep all of the attributes and let the data mining application select the meaningful ones. This process is known as attribute selection, or more commonly, feature selection.

As mentioned previously on the topic of missing values, EvRFind does not necessarily create rules that make use of all available attributes. In fact, because there is a built-in bias to create rules of minimal length, each one normally makes use of a very small number of attributes. Because this type of feature selection is more automated than in some systems, EvRFind does not explicitly deal with the problems associated with merging different datasets. This is described as a detail with respect to the representation used by EvRFind in Chapter 5.1.1: Rules, starting on page 68.

\subsubsection{Cyclical Data and Database Updates}

While the raw data being used as input for a data mining application might be static, in most cases the source database is dynamic. In the simplest examples there are constant additions to the data to reflect events such as recent sales transactions, but changes might also include modifications or deletions. 
The impact of changes to the source data will be determined by the accuracy of the predictive model on the new data. If the confidence is approximately the same, then it means that the original data is representative of the new data. If the confidence drops significantly then it may be an indicator of a poor model, but it could also indicate a shift in trend. These shifts can sometimes be attributed to cyclical data, such as a shift in buying patterns related to the season. Data mining systems often need to identify trends and ensure that the model reacts accordingly.

As with the case of noisy values, described previously in this chapter, EvRFind assumes that all of the data is valid and does not handle this problem explicitly. Although it is possible to train predictive models such that they are constrained to certain dates, or data cycles, this topic is outside of the scope for this research.

\subsubsection{Dataset Imbalance}

A problem that frequently occurs in classification tasks is dataset imbalance [Kubat, Matwin, 1997; Chawla et al., 2002; Drummond, Holte, 2003]. This is the case when one or more of the classes are heavily under-represented compared to other classes. As a result, there is not enough information present to model the unpopular classes.

This problem is well known in machine learning and a great deal of work has been done on this topic in recent years. Some of the techniques used to deal with this issue are to under-sample the popular classes [Drummond, Holte, 2003], to over-sample the rare 
classes [Chawla et al., 2002], and to use different costs based on class popularity as penalties for misclassification [Shin, Cho, 2003].

Imbalanced data can sometimes be representative of a domain, but at other times is the result of improper or restricted data collection or poor random sampling. Despite the reason for the imbalance it is an important problem because it occurs in many real-world applications. The implications are serious because even a model that boasts a very high predictive accuracy can be of little use in the case where the important classes are very rare.

In this research there are experiments done where the imbalance is inherent in the domain. In Chapter 6: The Poker Hand Dataset, starting on page 167, a synthetic dataset with this property is fully described. EvRFind uses generalization techniques to combat the problem of dataset imbalance, and it does reasonably well in the Poker Hand domain.

\subsubsection{Comprehensibility}

Understanding the results of a data mining system is critical to making proper use of them. Knowledge discovery implies that certain relationships or properties have been discovered, and although the root causes may not be fully understood, the relationships themselves are clearly documented. This is in contrast to a black box expert system, where a question is posed and an answer received, but the justification for the answer is not decipherable. 
Rule induction algorithms are an example of a system that creates comprehensible results. The simple nature of an if-then rule allows for easy analysis of relationships between attributes, values, or both. An artificial neural net (ANN) is an example of a system that is more difficult to understand. The ANN is comprised of a set of relationships between input and output values, where each relationship has a weight that has been trained. This constitutes a predictive model because it makes predictions based on various input values, but it does not qualify as knowledge discovery because the rationale for the weight of any given relationship is not clear. ANN is described in more detail later in this section.

During the development of EvRFind, comprehensibility was one of the primary goals for the system. The chapters to follow will show that starting with the original objective of discovering if-then rules, and then biasing the system towards making these rules shorter, and finally using abstraction and generalization techniques to create higher level constructs, EvRFind has consistently been improved with comprehensibility as one of the driving factors.

\subsubsection{Privacy Issues}

Another widely discussed issue raised by data mining applications is not technological, but social. Specifically, it is the issue of individual privacy. This concern stems from the fact that a data mining system has the ability to gather extensive details on an individual from a seemingly non-intrusive set of data. Although it may be a legitimate concern, this research considers only the technical aspects of data mining. EvRFind does not take 
external context into account during the data mining process, and thus, issues such as privacy and maintaining the confidentiality of data are outside the scope of this work.

\subsection{Existing Data Mining Techniques}

The most commonly used techniques for Data Mining are decision tree induction, clustering, visualization, and artificial neural networks. Other techniques, such as genetic algorithms and learning classifier systems, are less popular but it will be shown that each has had success in various domains.

Some of these techniques are used by EvRFind, while others are used by different systems with the same goals. For the latter case, these techniques are described in order to present a more complete picture when interpreting the results during the experimental evaluation.

\subsubsection{Decision Trees}

A decision tree is a predictive model that maps a set of values onto a conclusion through the use of simple, often binary, comparisons. There are different techniques used to perform decision tree induction, and two popular systems are C4.5 [Quinlan, 1993], or the more recent implementation C5.0 (See-5) [Quinlan, 1998], and the CART (Classification and Regression Trees) software [Breimam, Friedman, Olshen, Stone, 1984]. 
One of the methods that is used by the CART software is regression. Regression is a statistical technique that examines a numerical dataset and develops a formula that fits the data. The output is a decision tree that predicts continuous values. The limitation is that it typically works well with continuous quantitative data such as measurements, but often performs poorly on categorical data where the order is not important.

Classification is different than regression in that it handles a wider variety of data. The output is a predictive model that predicts discrete solutions. Solutions are in the form of a binary decision tree.

C4.5 is different than CART in that it uses a measure called Information Gain to determine where splits occur, and eventually which attributes are used to make decisions in the different levels of the tree. C4.5 employs additional techniques such as automatic tree pruning, handling of unknown values, and automatic rule generation.

EvRFind uses a combination of the techniques described here to create if-then rules. Although it is somewhat limited in its ability to create a model that predicts continuous values, the task has been achieved for several simple test problems that were used during the development of the Gen-Function. This functionality is described in Chapter 5.11.3, Gen-Functions, starting on page 127. 
EvRFind does not create decision trees, although there is a simple process to convert a set of if-then rules into a decision tree, and vice-versa. An example of this can be found in Chapter 5.5.3, Representing the hierarchy as a decision tree, starting on page 88 .

\subsubsection{Clustering}

Clustering is an unsupervised form of learning that partitions a set of data into smaller subsets, or clusters. The elements in the new subsets are grouped according to a distance measure, which determines how the similarity of two elements is calculated. The diversity of the elements in the original set will determine the number of clusters. This technique is more completely described in [Berry, Linoff, 1997].

In preparation for a classification task, clustering can be used as a technique for determining what the target classes are. Once complete, a predictive model can be built using existing data and new examples will be classified accordingly. The problem with this combination of supervised and unsupervised learning is determining what concepts the discovered classes actually represent. Depending on the information available concerning the domain, the technique might not qualify as knowledge discovery.

EvRFind does not specifically employ clustering as one of its techniques. However, analysis of the results in some domains has shown that the dataset becomes divided into cluster-like groups. Despite the similarity, this topic is not covered in the remainder of this work. 


\subsubsection{Visualization}

Visualization is a technique for creating tables, diagrams, charts and graphs to aid in the knowledge discovery process. This method works by converting data into various different forms, and then performing transformations and filtering. In many cases, seemingly abstract or random data will contain patterns that are easily discovered using the proper visualization technique. A well known example of this is RANDU, which is a linear congruential pseudorandom number generator created by IBM in the 1960s. Using visualization techniques it is easy to see, using a three dimensional graph, that all of the random points generated by RANDU fall within 15 planes.

One such visualization package is R: A Programming Environment for Data Analysis and Graphics [Becker, Chamers, Wilks, 1998]. This package is based on the S language, which is used for the statistical analysis of data.

Visualization software is most often used as an aid in the task of pattern recognition, and because EvRFind is an automatic rule discovery system, there is currently no capability for the user to mid-way interact with the system to influence the results. In the current version, EvRFind does not offer the types of charts and graphs that would be useful for an analyst to discover patterns in the post-processing stage. However, this capability may be realized in a future revision of the software. 


\subsubsection{Genetic Algorithms}

The use of GAs in data mining is described in Chapter 4: Literature Review, starting on page 53 .

\subsubsection{Artificial Neural Networks}

An Artificial Neural Network (ANN) is a non-linear predictive model that is trained by adjusting weights of relationships between input and output values. As with other methods, weights are adjusted through the use of example instances. A more complete description of the ANN can be found in [Berry, Linoff, 1997].

Several publicly described classification systems, such as [Duch et al, 1998], rely on artificial neural networks as a method for learning distinctions between examples and placing them into their appropriate classes. In several empirical studies the neural network has proven to be good for prediction. However, this does not necessarily mean that they are useful for knowledge discovery.

Prediction and knowledge discovery systems differ in the level of analytic detail that is presented to the user. In the case of prediction alone, even a model with $100 \%$ accuracy might reveal nothing of the underlying concepts. This is the notion of a black box, where answers to queries are provided without a description of how this decision was made.

In [Duch et al, 1998] the authors discuss classification using neural networks and conclude that knowledge acquired by such systems is represented in a set of numerical 
parameters and architectures of networks in an incomprehensible way. The authors go on to suggest that logical rules should be preferred over other methods of classification provided that the set of rules is not too complex and classification accuracy is sufficiently high. Their solution to this problem is a hybrid algorithm that extracts rules from the network after it is built.

EvRFind does not employ an ANN during the rule discovery process, although in Chapter 7: Miscellaneous Dataset Experiments, starting on page 228, there are comparative results against other systems that use this technique.

\subsubsection{Learning Classifier Systems}

A learning classifier system (LCS) [Holland, 1975] is a rule discovery system that utilizes genetic algorithms and reinforcement learning. The GA component creates and modifies the rules, while the reinforcement learning is used to adjust weights assigned by the environment for rewards or penalties.

LCS and its variants have been successfully applied to the task of Data Mining in a number of published results, such as [Gao, Huang, Rong, 2005]. In other published work, such as [Bagnall, Cawley, 2003], a classifier system is compared to other systems such as C5.0 decision trees, Artificial Neural Networks, and Support Vector Machines [Cortes, Vapnik, 1995; Burges, 1998]. It can be concluded that classifier systems are often outperformed by one or more of these methods. 
Due to the reported poor performance in published work that compares LCS against other techniques, EvRFind does not use LCS during the rule discovery process.

\subsubsection{Inductive Logic Programming}

Inductive logic programming (ILP) was first described in [Muggleton, 1991]. It is a combination of Machine Learning and Logic Programming used to create descriptions using example instances and background knowledge. The descriptions are represented as logic programs that can also be described by a set of if-then rules.

In terms of comprehensibility, EvRFind produces results that are similar to if-then rules that are generated by ILP systems. The techniques used by ILP are considerably different than those used by EvRFind, and are not discussed in more detail in this research.

\subsubsection{Stacking}

Stacking [Witten, Frank, 2005] is not an independent algorithm, but rather a meta-search technique. Meta-search refers to a system where the input is the combination of results from different models. Experiments have shown that combining predictions from several techniques will provide a more accurate prediction than any one individual method.

EvRFind does not make automatic use of this technique, but it is easily incorporated by supplying the system with results from another rule discovery algorithm. EvRFind will 
use previously discovered rules as a starting point for the evolution, and continue in an attempt to discover better solutions.

\subsubsection{Training and Testing Datasets}

Independent of the search method, the data mining process generates predictive models that have to be evaluated for accuracy. It is not sufficient to create a model based on all of the available data because the model can suffer from overfitting [Berry, Linoff, 1997]. An over-fit model is one that is trained to the point where it represents the training data very well, but makes specific predictions that are not correct in the more general case. This results in poor predictive accuracy on unseen data. The most important test for a new predictive model is to see how it performs on non-analyzed data from the same domain.

Several protocols have been developed to aid in assessing the value of a predictive model. Each of these protocols involves partitioning the data into training sets and testing sets. The training set is the segment of data used to create the model, while the testing set is used to check its predictive value. One common method for splitting and then evaluating datasets is named $N$-fold cross validation.

Cross validation is a multi-step algorithm that alternates between creating and reviewing rules. The input to the algorithm is the entire dataset and a value for $N$, which specifies the number of elements in each of the testing segments. The following steps detail the operation of the algorithm: 


\section{Retrieve dataset and the value for $\mathrm{N}$}

Split the dataset into $\mathrm{N}$ segments of approximately equal size (segments labeled 1 to $\mathrm{N}$ )

Loop through segments ( 1 to $\mathrm{N}$ )

TS := the segment indexed by the current loop counter value

TR := the collection of all segments excluding TS

Create a predictive model (M) using TR

Test (M) against all elements in TS and record the results

Tally and summarize the results

Running this algorithm produces $N$ predictive models, where the majority of predictions should be common to all. Infrequently made decisions are normally contradictory to the rest of the dataset and are given less weight than others. Associated with each model is the accuracy as determined through the evaluation, and the summary statistics provide information on the average accuracy over all models.

The size of $N$ often varies depending on the size of the dataset. There are a number of common choices, such as using 50-50. This specifies that half of the data is used for training, while the other half is used for the purpose of evaluation. The problem with this selection is that a random sampling of $50 \%$ may not be representative of the entire domain, and can lead to models that do not predict the data in general.

Another common choice is the leave one out method. This is the same process, but the value for $N$ is all of the elements in the dataset, minus one. Although this is a rigorous test of the algorithm for the given dataset, it takes considerably longer than the 50-50 method. 
Also important to mention is that despite the ratio or method chosen, it is important to ensure beforehand that the elements are not ordered in any way. To guarantee this, randomization of the instances within the dataset is recommended.

EvRFind was designed with an understanding that the practice of using training and testing datasets would be used in the majority of domains. As such, the system is set up to expect datasets that are split into these two types. Also, the majority of experimental evaluation for this thesis is done using this approach, and each experiment makes specific mention of the sizes of these datasets.

\subsection{Problems with Modern Rule Discovery Systems}

\subsubsection{Relationships between attributes}

Many learning algorithms, including some of the Data Mining techniques described here, do not take advantage of inter-attribute relationships. This is not to say that every algorithm assumes attribute independence in the same way that the Naïve-Bayes classifier [Rish, 2001] does. However, many algorithms do not search for or take advantage of these relationships when they exist.

In previous work, [Cattral, Oppacher, Deugo, 1999; Kusiak, Dixon, Shah, 2005] have shown that understanding the complexity of relationships between attributes has been beneficial, and perhaps necessary, in certain domains. 
EvRFind is designed to look for relationships between attributes, and will take advantage of them during the rule discovery processes. The results of this capability are shown in several of the experiments that are described in the chapters to follow.

\subsubsection{Limits in the representation language}

The representation language used by an algorithm is the basic grammar that describes knowledge once it is discovered. In the case of a rule discovery algorithm the grammar will minimally include an if-then structure, as well as several comparative operators.

In some domains, the representation language is not entirely compatible with the representation of the solutions. In published work describing the AQ17-DCI algorithm [Bloedorn, Michalski, 1996], the authors make use of functions that change the representation of the system. The grammar is essentially expanded, making it possible to accurately cover examples that could not be done abstractly. They describe this as a different way of looking at the same data.

This is more commonly known as constructive induction, which essentially is the creation of attributes that were not present in the original dataset. This concept is more completely described in Chapter 4.2: Constructive Induction, starting on page 63.

An example function for AQ17 is Addition $(x, y)$, where $x$ and $y$ are attributes in the same instance, and the function represents the sum of the two values. One major disadvantage to their system is that $A Q 17$ has a limited number of functions. It is possible to add more 
but the process is not automatic, and to be effective the user must have knowledge about the domain. If too many are added, especially if they are irrelevant, then the search space will increase unmanageably. This problem aside, however, it is a good example of how to expand the representation language.

EvRFind also performs constructive induction to overcome limits imposed by the representation language. This is achieved by using a library of primitive functions to create Gen-Functions. These high level constructs are described in Chapter 5.11.3, GenFunctions, starting on page 127.

\subsubsection{Building on previously discovered knowledge}

Another deficiency in many learning algorithms is the absence of a way to make use of knowledge as a stepping stone to more complex concepts. In most cases, a concept can be broken down into smaller, easier to find sub-components. A simple example is an English paragraph, where the most basic component is a letter or symbol of punctuation. After the concept of a letter is understood, more complex structures such as words and sentences can be discovered. To discover a sentence without having first discovered letters would be a considerably more difficult problem.

Any system that incorporates a covering algorithm such as CN2 [Clark, Niblett, 1989] builds on existing knowledge. This is done by first discovering general concepts, and then building on these using specialization operations during successive iterations. The 
more advanced concepts take their place alongside basic ones, ordered from more general to more specialized.

From an implementation standpoint, standard covering algorithms rely on a Hill Climbing search. This has known limitations [Webb, 1996] such as getting trapped by local maxima. In general, hill climbing is good as a local search because the search area during each step is restricted to the closest neighbours [Russel, Norvig, 1995], but does not necessarily do well starting from a random point in a large domain.

A stacking algorithm will use the results from several models, such as decision trees, neural networks, or rules generated using $\mathrm{CN} 2$, and determine how best to combine predictions to produce the maximum predictive accuracy. This is another example of using previously discovered knowledge to improve results.

EvRFind takes advantage of previously discovered knowledge through the use of a high level construct named the Meta-Variable. This is also a form of constructive induction, where the idea is that "good" discoveries are identified, possibly aggregated, and added to the representation language for use by other components during the search. This technique is described in Chapter 5.11.2, Meta-Variables, starting on page 119. 


\section{Chapter 4: Literature Review}

Chapter 2 introduced Genetic Algorithms and chapter 3 introduced Data Mining. This chapter starts by providing a literature review of the evolutionary algorithms, including genetic algorithms, for the task of rule discovery in data mining systems.

The second topic covered in this chapter is constructive induction. This concept is first defined, and a survey of the literature concerning constructive induction and data mining is presented.

\subsection{Evolutionary Rule Discovery}

While some published work exists on the topic of rule induction using genetic algorithms, the majority uses evolutionary computing as a supporting component. The first example of this is in [Duch et al, 1998], where the authors worked with the output of neural networks.

Unsatisfied with the lack of knowledge provided by the neural network approach, the authors suggested that a set of logical rules should be preferred over other methods of classification provided that the set is not too complex and classification accuracy is sufficiently high. 
The authors created a hybrid solution that extracts logical rules from the black box output of the neural network. The rules are ordered from the most to least popular, as in the case of a default hierarchy. The paper describes the results for several benchmark problems.

In [Cordon, Herrera, 1999], the authors describe an evolutionary process for designing Takagi-Sugeno-Kang (TSK) fuzzy rule-based systems. The motivation was the fact that in many problems it is not always possible to have human experts express their knowledge in the form of if-then rules, and thus important to develop a system to create these automatically.

The system uses a two-stage approach to first generate rules, and secondly to refine the rules created in the first step. The algorithm starts by generating an initial population using the knowledge that is available to the system. This is done in the hope that the search process will obtain good solutions more quickly. Next, the algorithm uses a combination of a rule induction algorithm and an evolutionary strategy to build a preliminary knowledge base from the training data.

In the second stage, the system uses a hybrid GA to modify the antecedent and consequent parts of each rule. This GA uses a real-number encoding scheme rather than a fixed length bit string, and the genetic operators chosen were previously known in the literature as working well for this encoding. 
Also, as part of the second stage, an evolutionary strategy is applied in an attempt to refine the rules during each generation. This is done during the recombination process, and is put to work with only an elite subset of the population.

In their later work, [Cordon, Herrera, Villar, 2001], the authors expanded the GA to learn more in the refinement step. In addition to modifying the values in the antecedent and consequent, the new system is able to add and delete terms as well. This makes the process more data-driven than in the previous version.

The second version also contained modifications to the crossover and mutation operators, as well as a new restart operation. The restart is performed if the population appears to be stuck on a local optimum solution, and involves regenerating many of the members in the population while maintaining the elite.

Described in [Araujo, Lopes, Freitas, 1999; Araujo, Lopes, Freitas, 2000] is GAPVMINER, which is a system that uses a parallel GA for rule discovery in a database. The motivation behind this work is to speed up the rule discovery process in very large databases.

Individuals in GA-PVMINER are if-then rules that are comprised of a variable length set of conjunctive terms in the antecedent, followed by a single term in the consequent. Each term is a simple structure of the form: <attribute, operator, value>, where the attribute refers directly to the database and the value is an integer. At the time of this paper the 
only operation supported was the check for equality. Combined with the restriction on values having to be integers, the system was only tested with categorical values, or where the attributes could be discretized and converted into a reasonable number of categories.

In the antecedent there is a user-configurable restriction on the number of terms, and because there is only a single operator allowed it meant that each attribute could only appear one time. The consequent is similar, but restricted further by allowing only a single term that contained the attribute for the goal-state.

In order to take advantage of several machines in parallel the population was broken into $N$ subpopulations, where $N$ is the number of goal states. The authors suggest that the value for $N$ match the number of available processors, otherwise it is possible that some goal states will not occur in any discovered rule. The work is distributed such that each processor works on the subset of rules that has the goal state in common. As a second point for breaking up the populations according to goal state, the authors claim that the system will benefit from the fact that only individuals with the same consequent can be recombined.

The first genetic operator, crossover, is designed to combine rules at their common attribute point, if it exists. For example, if two rules contain the same attribute "gender" then a single point crossover would occur at that point. In cases where the same attribute does not occur then one of the terms found in the first parent is copied into the second, 
and the crossover occurs at this point. The authors do not discuss cases where more than attribute is in common between parents.

The second genetic operator, mutation, has two variations. The first is called attribute mutation, where a valid attribute is randomly selected to replace the current one. Validity is determined only by whether it currently exists in another term within the antecedent. The second type of mutation is value mutation, where the value in the term is replaced with a randomly generated value found within the domain of the attribute on the left side of the term. The probability of either type of mutation is $50 \%$, once a term has been selected for mutation.

There is one additional genetic operation called removal. Based on the number of terms in the antecedent, there is a probability that one term will be randomly selected for deletion. The aim of this operation is to improve rule comprehensibility by shortening the antecedent.

In [Kokai, Toth, Zvada, 2002], the authors describe two genetic based learning methods named GEA and GeLog. The first algorithm, GEA, is a purely evolutionary algorithm that uses GAs. The second algorithm, GeLog, is a hybrid algorithm that combines GAs with ILP to create Prolog programs. These systems were compared against C4.5 and FOIL [Quinlan, Cameron-Jones, 1993] on two publicly available datasets. 
The representation used by GEA is a fixed length bit string, where one bit is assigned to each possible value of each variable. The mutation and crossover of the rule sets are performed by either inserting or deleting rules at random, or mixing the rules of two rule sets and then removing the duplicates.

In GeLog the mutation operators either introduce a new definition of the target concept, or delete or modify an existing one by adding, removing, or modifying the values or variables. There are different crossover operators that interchange the right hand sides of the rules, or subsets of terms between individuals.

The authors showed experimentally that the genetic learners achieve the classification accuracy of both C4.5 and FOIL, and in the case of more complicated problems they discovered models that were both simpler and more accurate. The same experiments also showed that the hybrid algorithm, GeLog, performed better than the standard GA implementation used by GEA.

For additional published research dating prior to 2002, a more complete survey can be found in [Freitas, 2002b]. Also found in this paper is information on the discovery of association rules. The author describes the difference between their discovery and classification tasks, in particular that more than one term can exist in the consequent.

The author also discusses feature selection, which is another pre-processing step that has been successfully applied using GAs in [Whitley et al, 1997; Yang, Honavar, 1997; 
Guerra-Salcedo et al, 1999; Kim G, Kim S, 2000; Jourdan, Dhaenens, Talbi, 2001; Rithoff et al, 2002; Fröhlich, Chapelle, Schölkopf, 2003; Morita et al, 2003]. An interesting note from the author in [Freitas, 2002b] is that a number of data mining algorithms, in particular algorithms that perform rule induction, already perform attribute selection when they discover a rule that contains a proper subset of the attributes. Essentially, in some cases, feature selection is simply a side effect. This effect also occurs in EvRFind because the discovered rules often do not contain all of the original attributes. This is described as a detail with respect to the representation used by EvRFind in Chapter 5.1.1: Rules, starting on page 68.

[Freitas, 2002b] also describes rule niching and the reasons that it is used by some systems. The application of rule niching is necessary to prevent the GA population from converging to a single individual. The only method described in cases where each member represents a single rule is a meta-search where the GA is run several times, each time with the intention of discovering a different target concept. EvRFind employs this technique in order to achieve more coverage in classification problems. This is described in Chapter 5.4.3: Improving Coverage, starting on page 82.

The author also describes data mining using genetic programming, both in the rule discovery and data pre-processing stages.

In [Minaei-Bidgoli, Punch, 2003] the authors describe using a GA for weighting feature vectors for classification. Rather than the standard bit string representation, their GA uses 
a real-value encoding to indicate the importance of each feature. The selection, crossover and mutation operations are typical for GAs with a real-value encoding.

The authors found that when using a small number of features, it was more beneficial to use feature weighting as opposed to feature selection. They also found that using multiple classifiers improved classification performance when compared to using any single classifier. After evaluation it was shown that using the GA to weight features improved performance by at least $10 \%$ in each of their examples.

Also mentioned earlier in the section on HGAs is a survey publication [Sinha, Goldberg, 2003] that discusses applications of decision tree induction and feature selection using hybrid genetic and evolutionary algorithms.

In [Tulai, Oppacher, 2004] the authors describe Multiple Species Weighted Voting (MSWV), which is a GA-based system used for classification. The system uses a decision tree to represent each individual, which means that each member of the population actually corresponds to several rules. In a method similar to that of a support vector machine, each rule is a binary (2-class) classifier.

Unlike the standard approach, this system does not use fixed values for the size of the population or the stopping criterion. Rather, these values are chosen after the search begins. 
The authors were unable to find an effective crossover technique, but instead use several types of mutation that are suitable for the decision tree representation. These include adding and removing nodes, swapping leaves, swapping internal nodes, swapping branches, and changing attributes. An attribute change depends on the data and can include changing a Boolean value to its complement, or changing the range of a numerical constraint.

The system uses a speciation mechanism to ensure that evolution takes all of the data into account, rather than focusing on particularly strong rules from the outset. This technique was shown to work with varying success depending on factors such as the size of the dataset and the types of attribute values.

When evaluating a particular instance the system uses a voting scheme to predict the class. Rather than use a combined vote, only the fittest individual in the subspecies is allowed to vote. The authors explain that this works because all of the individuals in a subspecies are all very similar due to the strong selection pressure.

In [Dryl et al, 2004] the authors present RuleFinder, which is GA-based system they developed for rule discovery in medical data. The rules are in the if-then format, where the antecedent is a conjunction of terms and the consequent is a single term. Each term is of the form: <attribute, operator, value>, where the operator must be one of equality, less than or equal to, or greater than. 
Each member of the population represents an individual rule. Internally each rule is represented using integers to indicate the attributes and operators, and a real value to indicate the right side of each term. Mutation is similar to that of a standard GA, while the crossover is restricted to choosing splice points between terms. The selection method used was fitness proportional, and did not include elitism.

Fitness evaluation for each rule relies on four criteria, and these are weighted according to an empirically derived scheme. The four components are: percentage of the database that matches the antecedent $(60 \%-80 \%)$, the percentage that matches the antecedent and the consequent ( $10 \%-20 \%)$, the length of the rule $(5 \%-20 \%)$, and a measure of how often the attributes directly correspond to those stored in the database $(5 \%-10 \%)$. Unfortunately, the authors were not able to further specify the weighting.

In [Gao, Huang, Rong, 2005] the authors propose LCSE, which is an extension of the classical learning classifier system (LCS) designed for the task of data mining. The classical LCS uses a GA to facilitate rule discovery and reinforcement learning to adjust the weights of discovered rules. The motivation to extend LCS was to avoid the problem of overfitting that is often apparent when working with small datasets. Also, classical LCS performs poorly with noisy or missing data.

Each member of the population represents an individual in the form of an if-then rule. The system uses a two-level approach made up of several LCSs for classification, and then a voting method used to combine the results into a final decision. 
The authors introduce a bootstrap module that is used to distribute the input into several sub-LCSs. The goal of this is to develop multiple sub-LCSs with different classification performances. The authors found that this improved the generality of the predictive model.

In [Gopalan, Alhajj, Barker, 2006] the authors describe a GAKPER, a GA-based postprocessing rule pruning strategy. The motivation for this comes from the fact that many data mining algorithms generate a large set of rules, and in many cases they over-fit the training set. The intention behind using the GA is to find the most accurate and most interesting subset of rules.

The GA uses a binary encoding scheme where each bit represents a rule in the original set. In the first stage the GA optimizes for efficiency, while in the second stage the GA optimizes using a measure of how interesting the rule is. The end result is a subset of the original rules that adhere to both objectives.

\subsection{Constructive Induction}

Constructive induction, sometimes referred to as attribute construction or feature construction, is a term that refers to the creation of attributes that do not exist in the original dataset. In general, the process is to apply operators to the original attributes in an attempt to create new attributes that are better suited to the representation space. This is required in some domains where the existing attributes are inadequate for the discovery 
of high level concepts. Essentially, the new attributes match the representation language of the problem, as opposed to the representation available using only the original attributes.

In EvRFind, the Gen-Function creation process makes use of this technique. This is described in Chapter 5.11.3, Gen-Functions, starting on page 127.

Gen-Functions are similar to new attributes derived through attribute construction in AQ17-DCI learning [Bloedorn, Michalski, 1996], where the language is extended based on regularities discovered within the training examples. Their technique, however, relies heavily on exhaustive testing in order to generate new attributes. Without a search space reduction task running beforehand, such as the way that EvRFind uses evolution to guide the search, this process is computationally too expensive to experiment with a large number of functions. Specification of which functions should be considered for attribute construction is the input required from the user.

In [Piramuthu, Sikora, 2006] the authors describe methods for using Feature Construction to enhance the learning process of a GA. Feature construction refers to the joining of two or more features to generate new features.

The feature construction process involves creating new features from combinations of existing attribute values using the standard set of arithmetic operators $\{+,-, *, /\}$. This takes place for several iterations, each time creating higher order polynomials. The order 
is ranked according to their success at discriminating the positive and negative example instances. After the ranking is complete, a certain number of features are selected and the process is repeated.

This process differs from EvRFind in that feature construction is done as a pre-processing step before the search begins. In EvRFind, constructive induction is guided by the evolution and occurs during the search process. This is described in Chapter 5.11.2, Meta-Variables, starting on page 119, and also in Chapter 5.11.3, Gen-Functions, starting on page 127.

Once the feature construction process is complete, the new set of features is used as input to the GA. Each individual in the GA represents a single rule and as in other published work, the antecedent is a conjunction of terms. The GA implementation is not described in detail, although it does mention that the fitness function assigns partial credit for partial matches as opposed to only awarding credit where the examples are properly classified.

In [Otero, Silva, Freitas, Nievola, 2003] the authors describe a Genetic Programming approach to attribute construction. The authors state the motivation for this concerns the quality of a data space representation. They continue to say that it is one of the most important factors influencing the performance of a data mining algorithm. Further to this, the attributes defining the data space can be inadequate, making it difficult to discover high quality knowledge. 
As in the case of [Piramuthu, Sikora, 2006], the attribute construction is done in the preprocessing stage, rather than during the search process. New attributes are limited to derivations of existing real-valued attributes, using only the standard set of mathematical operations $\{+,-, *, \%$ (protected division) $\}$ and a limited set of comparison operations $\{<=,>=\}$. The result is a new attribute in the form of a GP-tree, and can be used in conjunction with the original attributes.

The GP-tree is further limited by restricting the types of operations. If the root of the GP tree is an arithmetic operation then the new attribute will be real valued. Similarly, if the root of the GP tree contains a comparison operator then the attribute will be Boolean valued. These operations cannot be mixed in the same tree.

The GP-trees in this system are evaluated using a measure of information gain as the fitness function. The function uses different measure depending on whether the new attribute is real valued or Boolean.

The authors evaluate their system by running a number of experiments using $\mathrm{C} 4.5$, where half of the experiments use the original attributes, and the other half use the original attributes in addition to the newly created attributes. The results varied depending on the domain, but typically the addition of new attributes improved the predictive models derived using C4.5. 


\section{Chapter 5: EvRFind - Detailed Description}

EvRFind is a hybrid algorithm that uses components from both evolutionary and nonevolutionary learning systems. At the core of the system is a genetic algorithm based engine that makes use of features found in other methods including genetic programming and hill climbing. The search techniques are enhanced by other extensions that are used between evolutionary generations.

This intergenerational processing stage includes fine grained searching, optimization, automatic concept generalization, and automatic expansion of the description language. Essentially, what happens between generations is guided, or honed, by the evolutionary component.

To be successful at rule induction for a variety of datasets, EvRFind is required to be more accommodating than the standard GA. Following is a description of the components of EvRFind and how they differ from GAs or other learning techniques.

\subsection{Representation of Data}

The way that EvRFind represents data is one of the major differences between it and other GA-based systems. In a standard GA there is a fixed length binary string that represents each solution. A decoding function exists to convert this binary string to the appropriate form when required. It was determined at a very early stage that this representation is too restrictive for the predictive rules that are generated by EvRFind. 


\subsubsection{Rules}

The template for the type of rules being sought by EvRFind is:

If $\mathrm{X}_{1} \wedge \mathrm{X}_{2} \wedge \ldots \wedge \mathrm{X}_{\mathrm{n}}$ Then $\mathrm{Y}_{1} \wedge \mathrm{Y}_{2} \wedge \ldots \wedge \mathrm{Y}_{\mathrm{m}}$

The symbols $X_{1} \ldots X_{n}$, and $Y_{1} \ldots Y_{m}$ each represent terms within the rule. Within each set of rules, the values for $N$ and $M$ are not fixed, although they do have a specified maximum length. Each term can be used to indicate the existence of an attribute, perform a comparison operation on two variables, or execute a function with one or more variables as the input.

The standard GA representation cannot efficiently maintain the information required for each rule. The first problem is that solutions are normally stored using a fixed length representation. This would severely limit the structure of rules that EvRFind is attempting to discover. Although it is possible to specify an upper bound that is sufficient to hold an entire rule, there are many different sizes of rules within any given set. Accommodating this within a fixed length structure would require the use of NOP (no-operation) values, and will not be as efficient.

The alphabet used by a GA is called the set of terminals. In a standard GA there are only two of them, which are the digits zero and one. Variations of GA systems, such as Genetic Programming (GP) [Koza (1992)], use a much larger terminal set. Often the alphabet in GP is problem dependent and there are numerous terminals that represent both functions and variables. 
Rules are represented within EvRFind as dynamic structures that store the information required to present and evaluate the rule. EvRFind is similar to GP in this respect because it has both a user-defined number of terminals, and the length of the rule is flexible.

A flexible set of terminals is required in EvRFind because different datasets have a different number of attributes, and without accommodation for each of them it may not be possible to fully explore the data. The number of variables required can be less than the number of original attributes if not all of them are required to accurately describe the instance.

When only a subset of the original attributes are required then search efficiency can be improved through the use of a feature selection algorithm. This type of algorithm will attempt to identify attributes that are most likely to accurately discern the data. Because EvRFind constructs rules that use only a subset of the attributes, it essentially performs feature selection implicitly during the search. As pointed out in [Freitas, 2002b], this is often the case in rule induction algorithms that create rules containing fewer than the complete set of available attributes.

Furthermore, some datasets require more variables than attributes because derived values and functions are created. EvRFind also has the ability to expand the original set of terminals, and this is simply not possible when the framework is restricted by a fixed alphabet. 
Each rule is comprised of several components including the antecedent and consequent, as well as other data used for administrative and statistical purposes. Within both the antecedent and consequent there is a list of terms that makes up the corresponding portion of the rule. Each of these terms is further broken down into subcomponents.

\subsubsection{Terms}

A term always evaluates to a value of either true or false so that the if-then logic can be applied. Each term can optionally contain variables, constant values, a set of constant values, a Boolean negation operator, a comparison operation, or one or more functions.

\subsubsection{Term components}

\section{$\underline{\text { Variables }}$}

The variables correspond to attributes within the dataset. The relationship is not necessarily one-to-one between variables and attributes because the user can optionally ignore specific attributes. Variables have several properties including usage statistics, indicators for valid types and ranges, valid comparison operations, and an English name for reporting purposes.

\section{Constant values}

Constants have a limited role within the term. They are restricted to the right hand side of the term, and are only used for comparing against the value being represented by a 
variable, or against the return value from a function. Constants can also be used as parameters for supporting functions.

\section{Comparison operations}

EvRFind uses a standard set of mathematic comparison operators to compare values represented by variables or constants. The full set of operators available is: Equal to, Less than, Less than or equal to, Greater than, Greater than or equal to, and Not equal to. Variables specify the set of operators that are valid for the attributes they represent.

\section{Gen-Sets}

A Gen-Set is a special type that represents a set of values. It can be used in the place of a constant value and depending on the operation, has the ability to behave as several different constant values at the same time. The set can optionally be empty and represent no value. For detailed information on the Gen-Set operations, please refer to Appendix B: Gen-Set operations and properties, starting on page 297.

\section{Functions}

A function is an algorithm that accepts a combination of variables, and optionally constant values, to generate a new value. The logic encapsulated by the function is typically outside the scope of the standard comparisons that EvRFind uses. 


\subsubsection{Types of terms}

The subcomponents of a term are dictated by its type, which must be one of the following:

Boolean variable; this term indicates that a particular attribute exists, or does not exist, in the current dataset instance. This type is used in problems where individual attributes might not be contained in some records, such as during a Market Basket Analysis of retail transactions.

Variable compared to a constant; this term compares the value held by a specific attribute against a constant value and returns true or false.

Example: $(\mathrm{A}>5)$.

Variable compared against the elements within a set; this term compares the value held by a specific attribute against the elements within a Gen-Set, and behaves according to the specified Gen-Set operator.

Example: $(A=\{1,5,7\})$

Variable compared to another variable; this term compares the values held by two different attributes and returns true or false. The result depends on the operation and the relationship between values.

Example: $(\mathrm{A}<\mathrm{B})$. 
Simple function; this term executes a function operating on one or more values for input. The result is true or false.

Example: Is_Even(A).

Function compared to a variable or constant value; this term compares the result of a value-returning function to a constant value.

Example: $(\operatorname{Max}(\mathrm{A}, \mathrm{B}, \mathrm{C})>5)$.

Function compared to the elements within a Gen-Set; this term compares the result of a value-returning function to the elements contained within a Gen-Set using the specified Gen-Set operator.

Example: $(\operatorname{Min}(A, B, C)<\{7,12\})$

Function compared to another function; this term compares the results of two valuereturning functions.

Example: $(\operatorname{Add}(A, B)=\operatorname{Add}(C, D))$.

\subsection{Execution flow}

The next several sections within this chapter describe various processes and components within EvRFind. As an aid for understanding these concepts and how each fits into the larger system, an overview of the program execution flow is now presented in pseudocode. This represents on experiment from start to finish. 
Pre-processing

Data-file Statistics

Calculate reward constant

If this is a refinement then accept previous rules as input from user

Generate random rules to fill the population

Begin main loop

Loop: Evaluate fitness for each rule

Calculate raw fitness

If previously evaluated, skip calculation of raw fitness

Use confidence and support to calculate fitness

Apply fitness bonuses

Evaluate hierarchy-fitness based on position within hierarchy

Apply fitness-scaling adjustment

Check to see if the goals of the search have been completed

If targets met or problem solved, then exit

Trim population to pre-specified size if necessary

Elitism-copy "good" rules into next population

Loop: Create rules for next population

Select rule(s) with a bias towards greater fitness

Perform genetic crossover

Perform genetic mutation

Discard invalid rules if necessary

Add rule(s) to next population

Perform intergenerational processing 
Rule validation and optimization

Check for and modify or remove invalid rules

Check for inefficient rules and optimize or remove

Analyze population and create Meta-Variables

Search good rules for similar terms

If found, group terms and create Meta-Variable

Replace the terms that were used to create the Meta-Variable

Add the Meta-Variable to the language

Analyze population and create Gen-Functions

Search good rules for common themes using primitive functions

If found, create a Gen-Function to generalize the knowledge

Create a set of rules that will express the Gen-Function

Add the new rules to the next population for evaluation

Add the Gen-Function to the language

Perform local search (Hill Climbing)

If refining, select most fit rule, not previously selected, as target

If exploring, run tournament selection to select target rule

Perform mass-single-mutations to create new rules

Add new rules to the next population for evaluation

Mark target rule as "Hill Climbed" to avoid future selection

Update ancestral history for lifetime duplication check

Post-processing

Report results 


\subsection{Pre-processing stage}

The pre-processing stage of each experiment is used for work that is usually completed a single time for each dataset. However, if significant changes are made to the parameters of the experiment then any pre-processing can be done immediately before the experiment starts.

\subsubsection{Data-file Statistics}

In the current version, the only routine in use during the pre-processing stage is the Datafile statistics (DFS) module. The DFS module creates a file that is associated with a set of example records, such as a training set or testing set, and contains statistical information about the data. These statistics include:

a) The total number of example instances in the file.

b) The total number of comment lines.

c) The total number of blank lines.

d) The number of attributes.

e) The number of instances that each of the attributes are used in.

f) A list of the distinct values for each attribute.

g) The frequency of each value that is represented.

These statistics are compiled by parsing the example instances that will be used during the experiment. Because the process can be time consuming, a DFS file is only created 
once for each dataset. Not having to recalculate statistics every time is more efficient, but a check had to be put in place to detect changes in the dataset.

In order to preserve integrity in the statistics, the DFS file also stores a digital signature that is unique to each dataset. When a new experiment is started with the same data then the signatures are compared. If necessary, the DFS file will be automatically re-created before the experiment continues. This check removes the chance of error that might occur if the dataset is intentionally modified or becomes corrupt.

Information in the DFS file is used throughout the experiment. The first reference is at the start of the log for each experiment, where a summary of the records is presented for reporting purposes. More significant is the use of statistics during the course of the experiment for the purpose of calculating a reward constant. This will be more completely explained later in the section under 5.4.4: Fitness Rewards, starting on page 83.

\subsection{Fitness Function}

In order for a GA-based system to effectively search for solutions, an appropriate fitness function must be implemented. A fitness function is an algorithm that examines a valid solution and calculates a numerical score to illustrate how close it is to the goal.

In a data mining system, the fitness function will ideally give an accurate measure for how close the rule is to what is being sought. Because this measure is subjective, 
particularly in undirected mining tasks, there is no straightforward way to implement such a measure.

With only a limited amount of information available for each potential rule, an effective fitness function has to combine as much of this data as possible. In EvRFind, the raw fitness is determined by using the values for confidence and support. Depending on the task at hand, the user can select an option that will combine the confidence and support factors appropriately.

The deciding factor for which fitness function to use is whether the search is supervised or unsupervised. Because these goals have several differences it is worthwhile to distinguish them slightly, where each has default options in place to optimize the type of search.

\subsubsection{Custom confidence and support factors}

For an undirected search it is important to be able to set targets for confidence and support, and then have EvRFind search for rules with those characteristics. Rules are evaluated and assigned a raw fitness using the following equation:

fitness $=100-\sqrt[2]{(\text { confidence }- \text { confidence Target })^{2}+(\text { support }- \text { supportTarget })^{2} \text { sfAdjustment }}$ 
This equation converts the values for confidence and support into a single numerical value that increases as each factor approaches the respective target. Basically, the closer the values are to the goals, the higher the fitness is for the rule.

The confidence and support target values are stored with the parameters that are specific to each dataset. These target values are only used for non-classification problems, and have default values of $85 \%$ confidence and $15 \%$ support.

An optional adjustment can be applied to change the importance of the support factor with respect to the rule confidence. This extension is the last term in the equation, and is called the SF-Adjustment (support factor adjustment).

By default the SF-Adjustment is 1.0 , which means that both confidence and support factors are weighed equally when calculating the raw fitness. Depending on the dataset and the domain it might be important that a higher confidence is preferred to a higher support. In these cases, entering a decimal value greater than zero but less than one will lower the impact of the support factor. Conversely it is possible to raise the impact of the support factor by entering a multiplier value that is greater than one.

It is important to remember that this equation derives a value for raw fitness only. Fitness is later modified based on several problem-specific parameters. These parameters are fully described later in section 5.4.4: Fitness Rewards, starting on page 83. 
One advantage to deriving the raw fitness from the confidence and support factors is that it selects cross-sections of data in an unbiased manner. In theory it is possible to substitute the fitness function for one that scores rules according to an underlying theme that is related to the problem, or a relevancy hierarchy that contains additional background information. The problem with this approach is that a human expert originally configures it, and the same expert's bias extends through to the final results. When using confidence and support factors the only human interaction is the specification of the target levels.

One of the disadvantages with using this fitness function is the impact that it has on classification problems. To illustrate this point, consider the example of a market basket analysis. In this type of problem it is logical to think of confidence and support being weighted equally because the goal is often to find fairly confident but fairly infrequent combinations. In classification problems, however, it seems more important that a rule be $100 \%$ confident. The confidence should not be weighted so high as to give rules that answer single outlying instances more fitness than rarely inaccurate rules that answer dozens of instances. In terms of the hierarchy this might mean that outliers are near the top of the list, rather than at the bottom, which is where they might lay when using equal weighting between confidence and support.

One way to achieve this is to lower the support factor target value specifically for classification problems. An example of how this approach was successfully used in practice is available in Chapter 7.5, Tic Tac Toe Dataset, on page 249. However, to better 
handle these supervised searches, a second fitness function is available. This option, named Classification Fitness, uses a different calculation for raw fitness that includes specific goals and adjustments.

\subsubsection{Classification fitness}

For supervised classification problems where the classes for each example instance are provided for training, the goal is to maximize the confidence of discovered rules. This guarantees that the rules will be as accurate as possible, as often as possible. Similarly, in most cases, the goal in a classification task is to discover the most general rules. Rules that cover a large number of instances have a large support factor, and thus these rules can be discovered by maximizing the support target.

The main goal of the classification fitness function is to maximize predictive accuracy as much as practical, and then concentrate on maximizing coverage. To accommodate this in EvRFind, a control called the Search Goal exists on the main screen. This control is used to specify classification problems (labeled Maximize confidence and support) versus non-classification problems (labeled Custom C/S settings).

The equation to calculate the raw classification fitness is:

fitness $=($ confidence $* 0.7)+($ support $* 0.3)$

This is a much simpler equation, where $70 \%$ of the fitness is contributed by the confidence, while the remaining $30 \%$ is contributed by the support. As in the case with 
non-classification tasks, there can be several bonuses added to the raw fitness. These are explained later in the section at 5.4.4: Fitness Rewards, starting on page 83.

\subsubsection{Improving Coverage}

Also of interest for classification tasks is the data coverage. A high coverage means that most, or perhaps all, of the examples in the dataset are being properly classified by the predictive model. There is an option available, Encourage wider rule coverage, which is beneficial because it rewards rules for uniqueness with respect to the example instances that they answer. Associated with each rule is a support percentage, which indicates the percentage of instances that the current rule is the first to correctly classify.

The first experiments with EvRFind used the following calculation to penalize rule fitness for redundancy:

fitness $=($ fitness + supportFactorPercentage $) / 2$

The averaging of the fitness against the support percentage worked reasonably well, although it had a problem in cases where a rule answered very few instances with $100 \%$ confidence. The outcome was that the fitness was boosted unfairly, and an inappropriate fitness was achieved. To address this issue, the fitness adjustment was changed to the following:

fitness $=$ fitness $*($ supportFactorPercentage $/ 100)$ 
The new equation scales fitness according to how much of a difference it makes within the population. Trivially, if the rule addresses no instances that aren't handled by a better rule, then the fitness is zero. To the contrary, if a rule is very good and is the first to answer all of the examples it covers, then the fitness will not be adjusted at all.

\subsubsection{Fitness Rewards}

A fitness reward is a bonus that is applied to the raw fitness, and is used to assign extra credit to rules with particular properties. Reasons for receiving such a bonus include parsimony and the use of variables instead of constants inside terms. The reward mechanism also includes assigning a bonus for the use of Meta-Variables and GenFunctions, both of which are explained later in the chapter.

\subsubsection{Reward Constant definition}

EvRFind uses a system wide variable called the reward constant. This is a base value that is added to the raw fitness as a bonus for a variety of reasons that are explained in more detail in the next section.

When this value was first incorporated into EvRFind it was a fixed value that did not change, except through manual configuration by the user. This meant that it did not automatically scale regardless of the size of the dataset. A serious problem with this was observed when experimenting with datasets that contained very large numbers of examples. 
As experiments were performed with larger datasets it was noticed that the precision of the fitness becomes more significant. For very large datasets it meant that the bonus had a more pronounced effect, and this sometimes had a negative impact on the results.

For these cases it meant that the reward constant should be lowered, but this additional configuration step adds maintenance and introduces the potential for error. Automation of this became possible with availability of statistics in the DFS file, which was described in Chapter 5.3.1: Data-file Statistics, starting on page 76.

The work done during the pre-processing stage allows for the reward constant to be derived from the DFS file, and this varies according to the size of the dataset. Based on several experiments with datasets of different sizes and complexity, the optimal formula for the calculation of this value was found to be:

reward constant $=(10 /$ number_of_example_instances $)$.

This value is large enough that the application of fitness bonuses will properly re-order two semantically equivalent rules. However, the bonus is not large enough to improperly displace rules that are genuinely better in terms of their confidence and support targets.

\subsubsection{Reward Constant usage}

Adjustments made to fitness were the subject of another group of experiments, with the following equations yielding the best results. 
a) (Reward constant * 7) for each term difference between the current length and the maximum allowed length. This bonus is intended to award credit to rules for being parsimonious.

b) (Reward constant * 2) for each term that contains a comparison to a variable, rather than a constant value. This encourages the discovery of relationships between attributes.

c) (Reward constant* 2) for each use of a Meta-Variable. This encourages the use of building blocks in the expansion of the representation language.

d) (Reward constant * 2) for each use of a Gen-Function. This encourages the use of generalization functions.

Several other rewards were evaluated and discarded when found to produce negative results. An example of this was a reward to encourage more complex Meta-variables by assigning a bonus for each encapsulated term. Although the idea of having more complex building blocks may have merit, this approach had the side effect of Meta-Variables growing uncontrollably with neutral terms. Rather than search for a way to ensure that Meta-Variables could only grow if benefit was realized, this reward was removed. 


\subsection{Evolving and Using a Default Rule Hierarchy}

\subsubsection{Description}

One of the characteristics of EvRFind is that it evolves rules in the form of a default hierarchy, with the emphasis placed on creating a strong group rather than strong individual rules. Using this approach, rule sets are kept simple in both individual rule complexity and the total number of rules that are required.

A default hierarchy is a collection of rules that are executed in a particular order. Whenever a new element is compared against the list, the rule at the top is tried first. If the antecedent correctly matches the data then the rule is used. If the data does not satisfy the antecedent, the next rule in descending order is compared. This continues until a matching rule is found, or the rules in the hierarchy are exhausted.

The traversal through rules is identical to the evaluation of nodes in a decision tree. However, in a decision tree the logic is limited to a single term for each level. One rule effectively represents an entire path through a decision tree. If a complete rule hierarchy is created then it will cover every path starting from the root of the tree to each of the distinct terminating nodes.

In [Clark, Niblett, 1989] the authors describe the decision list that is implemented for use in their system, CN2. The default hierarchy is a similarly ordered set of rules that also performs similarly when evaluating unseen instances. The main difference between the default hierarchy and the decision list is the way in which the set of rules is compiled. 
Further comparison against the decision list is outside of the scope of this research, but a more complete description is available in the referenced paper.

\subsubsection{Example}

Using a default hierarchy has the advantage of being able to obtain perfect accuracy overall, without each rule having to be $100 \%$ confident. The higher levels of the hierarchy play an important coverage-extending role by protecting less confident rules, as in the following example:

$1:$ If $($ size $>8)$ then class $=$ large

2: If $($ size $>2) \wedge($ size $<=8)$ then class $=$ medium

3: If $($ size $>0)$ then class $=$ small

In this example it is important to realize that the first two rules are independently correct, and can be transposed without affecting the results. The third rule is different because it is position dependent, and is only able to accurately answer those elements not addressed by the first two rules. In this example the only available values for size in the third rule are 1 and 2 because values outside that range were already excluded. If this rule were used in isolation on the same dataset then it would result in a large number of misclassified elements.

Although not obvious in the simple hierarchy shown here, experimentation has shown that rules at the top of the hierarchy cover most of the data, while those near the bottom 
often handle exceptional cases. Unlike a set of independent rules, where each exclusively answers a subset of data, the rules discovered by EvRFind are typically simpler and have fewer constraints because they can rely on the protection and support from others.

Although there is often some overlap between elements it is only the first matching rule that is used, and no ambiguity is introduced into the results if this occurs.

Depending on the dataset, this hierarchical approach normally requires fewer and less complex rules to successfully classify the data. This is partly because simpler rules are faster and easier to construct, so they tend to dominate the population early in the search. Also, when compared to a very specific rule, each of these will attempt to classify a larger number of instances.

\subsubsection{Representing the hierarchy as a decision tree}

As described earlier, the logic applied to rules within the hierarchy is similar to the evaluation of nodes in a decision tree. Some decision tree algorithms, such as C4.5 [Quinlan, 1993] include the ability to automatically convert the decision tree into a set of if-then rules.

The reverse process, converting a set of rules into a decision tree, is possible but may require some optimization to remove redundant or single-child nodes. Consider the example in the previous section. 


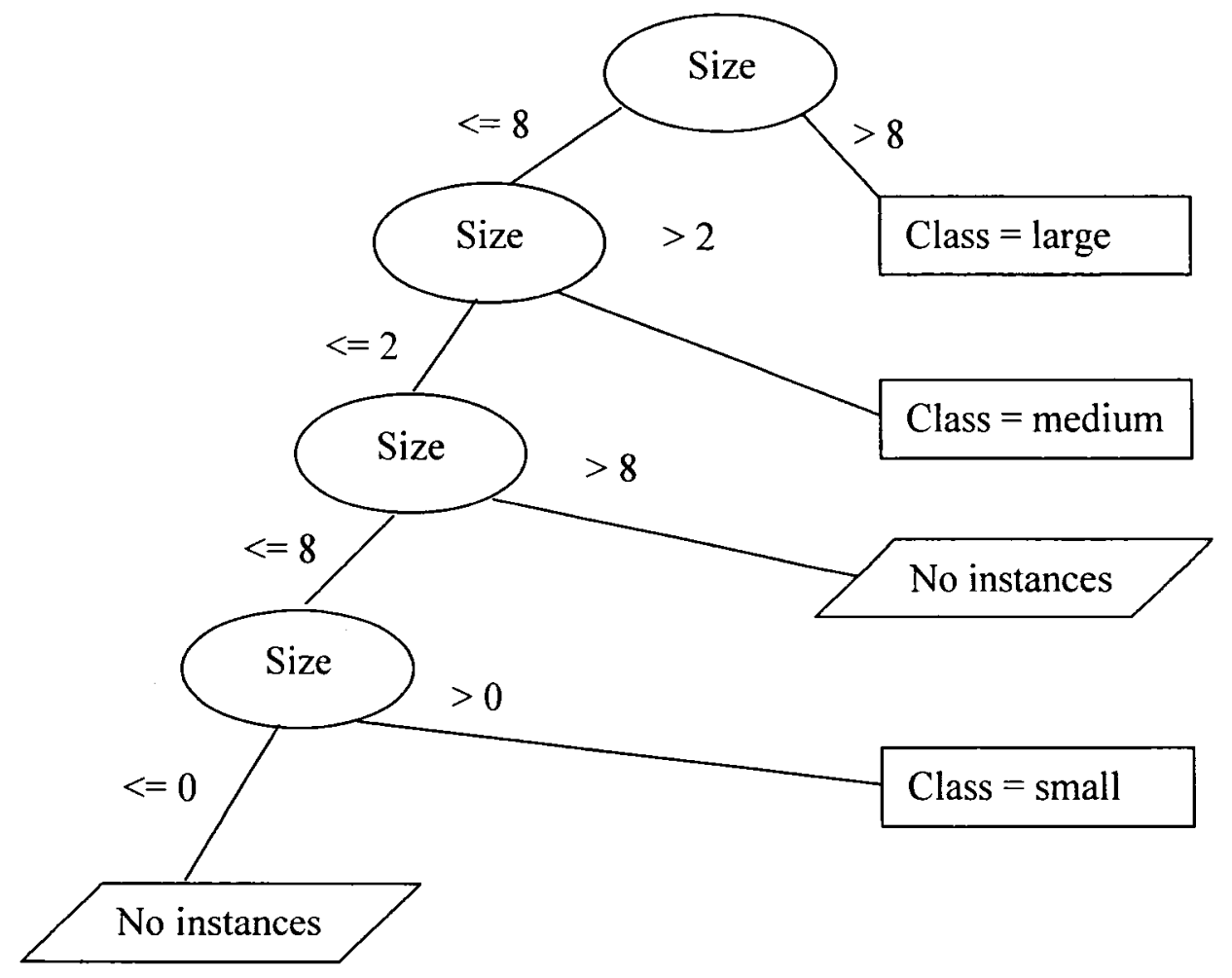

Figure 5.1: Decision tree representation of a default hierarchy

The extra step required to optimize this decision tree would be to remove levels that have one child that is never used because it doesn't classify any instances. In the first case, at the third level, this occurs because the comparison for $($ Size $<=8)$ is already done at the first level. In the second example, at level four, this occurs because there are no elements with a size of zero or less. In the case of rules it is sometime necessary to use this level of specification, but can easily be removed when representing this model as a decision tree. 


\subsubsection{Dealing with overfitted rules}

Because maximizing data coverage is one of the goals when evolving a hierarchy, it follows that over-fitting the solution set is a potential problem. An over-fit set of rules is one that has a very high accuracy when evaluated on the original training set, but is not general enough to accurately answer unseen examples from the same source. This problem is taken seriously in classification tasks, and mechanisms are generally in place to deal with it.

Two popular ways to curb the effect of over-fitting are pruning the results and relaxing the constraints. When a decision tree is pruned it has the effect of grouping similar items together based on their position within the tree. Similarly, relaxing the constraints is done such that more general cases apply to each available class. The problem with these methods of generalization is that they come at the cost of trading-off predictive accuracy. Although generally accepted, this type of solution also creates new problems because some cases simply cannot be classified accurately as a result.

In EvRFind, no trade-off is made between predictive accuracy and maintaining more general rules. Instead, the default hierarchy is used to identify and resolve cases of overfitting. This is possible because rules that answer very few elements are in the lower segment of the hierarchy, and can be easily identified as outliers or other rarely occurring cases. For example, a very unpopular rule might be one that handles only one or two out of perhaps five thousand example instances. 
It is important to note that this type of over-fitting is harmless because these rules are only tried as a last resort. If all of the general rules are compared and no match is found then the element still might be classified by one of the exception cases. This is not the same as using a default class, where an element is placed into a popular group simply because it could not be identified otherwise.

\subsubsection{Using a default class}

Assigning a default class is often done as a last resort in order to extend coverage. In a decision tree, the procedure is to simply assume that unclassified elements each belong to the class that holds the majority of elements at the previous node. In many cases this successfully extends the coverage, but the only basis for applying it is that one class is more popular than the others. Obviously, when dealing with subsets of data, this will not always be the case. In EvRFind it is still possible to use a default class by setting it as the last rule in the hierarchy. In this position the class will be assumed only if there is no match when compared against every other rule.

The only drawback to using a default class, or a rule with very limited coverage, is that in some domains it is less costly to leave the class unknown rather than to misclassify it. In these cases a default class should not be used, and in EvRFind it might involve scaling back the hierarchy such that the bottom segment is not used. For example, arbitrarily choosing to use only the top $80 \%$ of rules might leave several items unclassified, but has the potential to greatly reduce the problem of over-fitting. 


\subsubsection{Building the hierarchy}

In EvRFind, there are two ways that a default hierarchy can evolve. In the first case it is created as a side effect of the rule discovery process, while in the second case it is explicitly evolved.

During the development of EvRFind there was no initiative to explicitly build a default hierarchy. The set of rules orders itself as such because they are sorted by fitness, and fitness is a combination of the confidence and support. In practice, the rules are inserted into the hierarchy and their position can change based on other rules that co-exist within the set. The result is an ordered list of rules that makes sense when unseen instances are evaluated as if it were a default hierarchy.

When the default hierarchy began to evolve naturally, some experimentation was done in order to see what benefit might come from explicitly trying to evolve the model in this way. These efforts lead to the implementation of a specific feature, Evolve for a default hierarchy, which can optionally be used to force EvRFind to aim for a default hierarchy during the rule discovery process. This was feasible because of the flexible fitness function, and the availability of values for both global and hierarchy fitness.

\subsubsection{Global Fitness versus Hierarchy Fitness}

The global fitness is a combination of the global confidence and the global support. Similarly, the hierarchy fitness is a combination of the hierarchy confidence and hierarchy support. The "global" specification refers to the fact that the values do not take 
other rules into account, while the "hierarchy" specification indicates that the values may be adjusted based on the position of the rule within the group. The following example, adapted from the one shown earlier in this section, highlights the difference between these two concepts.

1: $[\mathrm{C}: 100.0(100.0), \mathrm{S}: 35.0(35.0)]:$ If $($ size $>8)$ then class $=$ large

2: $[\mathrm{C:} 100.0(100.0), \mathrm{S}: 35.0(35.0)]:$ If $($ size $>2) \wedge($ size $<=8)$ then class $=$ medium

3: [C: $30.0(100.0), \mathrm{S}: 30.0(30.0)]:$ If $($ size $>0)$ then class $=$ small

In this example hierarchy the following template description applies to each of the rules:

[C: Global confidence (Hierarchy confidence), S: Global support (hierarchy support)]

Because each of the first two rules is independently correct it follows that the global confidence is $100 \%$ for both. Similarly, because their position in the hierarchy has no effect on either rule, the hierarchy confidence is also $100 \%$.

The third rule is different because in isolation it answers $70 \%$ of the instances incorrectly, which leads to the global confidence of $30 \%$. However, because of the protection offered by the two previous rules in the hierarchy, the rule accurately covers all of the remaining instances in the dataset and consequently the hierarchy confidence is $100 \%$.

The values for global support and hierarchy support work similarly, where the hierarchy support indicates how often the rule is used when taking the position into account. 
Certain properties of the rule can be determined by comparing the global confidence (Gc) to the hierarchy confidence $(\mathrm{Hc})$ within the same rule.

a. $\mathrm{Gc}=\mathrm{Hc}$ : This rule is not dependent on other rules within the hierarchy. If, for example, both of the confidence values are $100 \%$ then the rule is always going to score $100 \%$ when evaluating the rule with the same dataset.

b. Hc $>$ Gc: The rule relies on the protection of other rules that precede it in the hierarchy. As a result, if a rule with this property were suddenly placed at the top of the hierarchy then it will not contribute as strongly to the model. In many cases, using a rule with this property "out of order" will have a detrimental effect on the overall predictive accuracy. At the same time, however, it plays a very important role when certain instances from the dataset are classified and removed before this rule is applied.

c. Hc < Gc: Some of the instances that would have been correctly classified by this rule were already answered, either correctly or incorrectly, by a rule (or rules) at a higher position within the hierarchy. This property can act as an indicator for how much overlap there is between the instances covered by certain rules. More general rules that are several steps from the top of the list will tend to show a greater ratio between $\mathrm{Hc}$ and $\mathrm{Gc}$. 


\subsubsection{Method for specifically trying to induce the default hierarchy}

As mentioned above, the default hierarchy is emergent rather than planned, and an option was implemented that specifically aims to create a default hierarchy. This option uses the values for hierarchy confidence and hierarchy support, rather than the global values, to calculate the fitness. The process is similar to what happens in a covering algorithm, where instances are removed as soon as they are matched by any rule.

With the fitness calculated using the hierarchy-adjusted values, the population evolves with the implicit knowledge that there may be dependencies on other rules, or on a specific ordering. It was expected that this would allow the default hierarchy to evolve faster, and perhaps yield a more effective model than relying on self-organization alone.

Evaluation of the Evolve for a default hierarchy option is performed on the Poker Hand dataset and summarized in Chapter 6.7.3: Evaluating the Hierarchy Protected Fitness option, starting on page 200. Experimental evaluation is also done on several datasets in Chapter 7: Miscellaneous Dataset Experiments, starting on page 228. The results of these experiments will be summarized in that section, as well as in the conclusion of this thesis.

\subsubsection{Losing coverage between generations}

One of the original goals of using a default hierarchy in EvRFind was to eliminate the possibility that a model will become less accurate from one generation to the next. The basis for this is that a genetic algorithm running with elitism will never lose the best member of the population. Therefore, if the best $N$ members of the population are 
maintained such that they can only be replaced by better solutions, then in theory the solution should never get worse as it evolves from one generation to the next.

During the development of EvRFind, a case was observed where the coverage actually decreased slightly between generations. Further investigation showed that it was a very rare occurrence, although it was possible and is illustrated by the following example.
If $(\mathrm{A}>\mathrm{B}) \wedge(\mathrm{C}>\mathrm{D})$ Then $($ Class $=1)$
[100\% global confidence]
If $(\mathrm{A}>\mathrm{B})$ Then $($ Class $=2)$
$[100 \%$ hierarchy confidence]

Both of these are $100 \%$ confident because of their ordering in the hierarchy. The second rule catches instances that are less specialized than those addressed by the preceding rule. If between these two rules the entire dataset was being correctly classified then the search would halt. However, for the sake of this example, it should be assumed that a more detailed model is required.

Consider the case where the first rule is modified or replaced by a superior rule. Given that the rule confidence is already $100 \%$, this will only be possible where it can be replaced by a rule that either has better support, or is given a slight advantage due to one of the fitness bonuses such as parsimony. Most importantly, consider the following case where the first rule is replaced by one that no longer protects the second rule. 


$$
\begin{array}{ll}
\text { If }(\mathrm{A}>\mathrm{B}) \wedge(\mathrm{C}=\mathrm{F}) \text { Then }(\text { Class }=1) & {[100 \% \text { global confidence }]} \\
\text { If }(\mathrm{A}>\mathrm{B}) \text { Then }(\text { Class }=2) & {[20 \% \text { hierarchy confidence }]}
\end{array}
$$

After evolving to the new form, where one term changes from $(\mathbf{C}>\mathbf{D})$ to $(\mathbf{C}=\mathbf{F})$, the first rule is still $100 \%$ confident but it covers more instances than it did previously. Some of the new instances that it covers were previously being covered by the second rule. Not all of the instances necessarily overlap, but the rule still appears better because the support is higher.

As the current generation evolves to the next, the second rule may be demoted because it has lost some of the instances that it previously received credit for. Eventually, depending on the nature of the dataset, many rules could eventually take position between the two original rules. Consider the following future hierarchy:

If $(\mathrm{A}>\mathrm{B}) \wedge(\mathrm{C}=\mathrm{F})$ Then $($ Class $=1) \quad[100 \%$ global confidence $]$
$\{$ several rules $\}$
[varying confidence]
If $(\mathrm{A}>\mathrm{B})$ Then $($ Class $=2)$
[ $5 \%$ hierarchy confidence]

In the event that this occurs, the noted several rules that now take priority over the original second rule are still not perfect. Although these rules perform well enough to maintain their position within the hierarchy, each one still misclassifies certain instances. 
Specifically, the instances that are being misclassified were previously being handled correctly by the second rule. However, now that the second rule no longer has priority, these instances are never properly classified and there is an overall decrease in coverage.

Fortunately, in all of the experiments that were done, this problem proved to be an intermediate setback. Through a combination of classification elitism, fitness

proportional selection and genetic crossover, the important components of each rule were eventually combined to make better aggregate rules. The final result was a population of rules that served as a better predictive model than in any of the previous generations.

\subsection{Evolving From One Generation to the Next}

Starting from the initial population of solutions, a GA evaluates and evolves individual members so that they can be added to the next generation. Once complete, the process starts again with the more-evolved group and the process continues. The following chart outlines the process. 


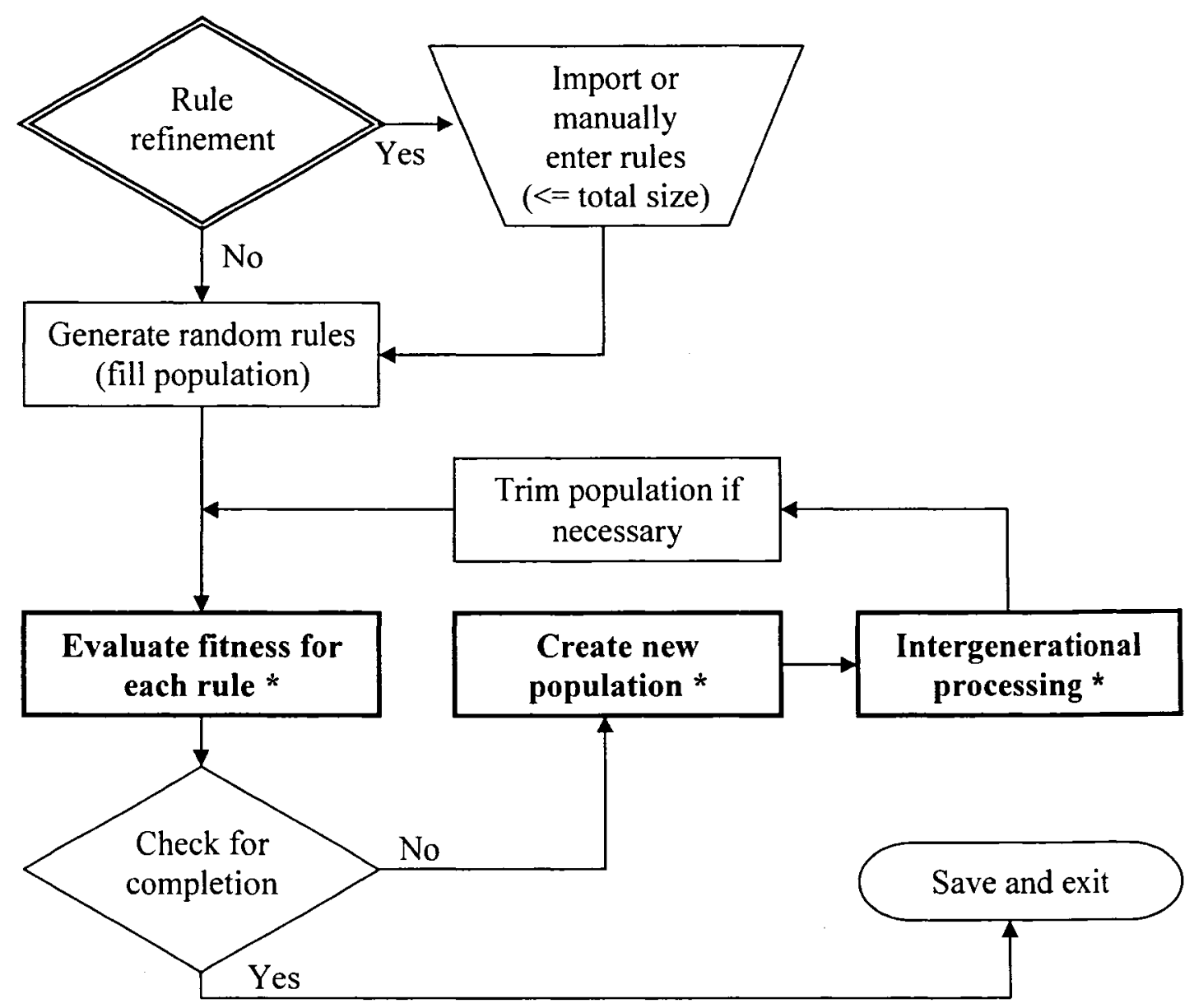

Figure 5.2: Single experiment overview

$(*)$ : The processes labeled "Evaluate fitness for each rule", "Create new population", and "Intergenerational processing" each expand and are presented as separate diagrams that contain details specific to that process.

Flowchart expansion reference

Evaluate fitness for each rule - Page 100

Create new population - Page 110

Intergenerational processing - Page 112 
The following chart is an overview of the fitness evaluation process, which was previously described in section 5.4, Fitness Function, starting on page 77.

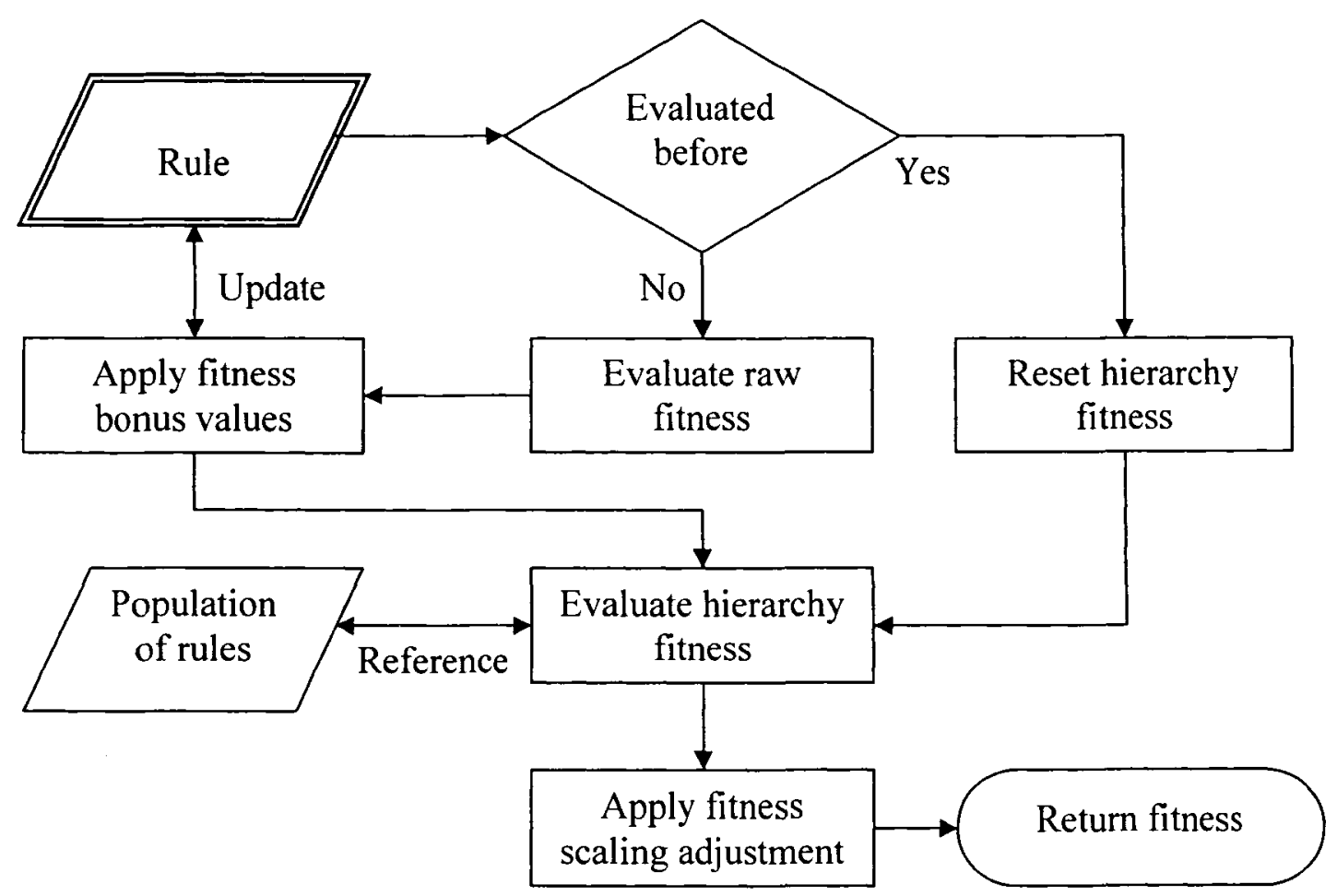

Figure 5.3: Rule fitness evaluation

This chart is the continuation of a previous chart, the Single experiment overview, on page 99.

\subsection{Elitism}

Because GA-based systems are non-deterministic, there is no explicit guarantee that good members remain within the population after each generation. For EvRFind, this means 
that although there is a high probability that good rules will continue to thrive in future generations, it is not impossible that one or more are lost and not rediscovered.

The loss of good rules between generations means that the average or maximum fitness can actually decrease during evolution, which is essentially taking a step backwards in terms of developing a hierarchy. Particularly in the case of classification, where the goal is to discover a set of rules that achieve perfect coverage, loosing a good rule can be a serious setback. To avoid this potential problem it is necessary to use elitism.

Elitism is a process that guarantees certain rules are copied directly into the next generation without having to be probabilistically selected. These copies are not subject to crossover or mutation, and thus will appear in exactly the same form.

Although there are several versions of elitism that are regularly used by GA-based systems, the standard methods are too limited to handle the representation used by EvRFind. The main reason for having to re-think elitism was that other forms only preserve the top $N$ members according to fitness, where $N$ is normally one. While some systems benefit from copying these groups of highly fit individuals between generations, EvRFind gained very little in some cases because of the hierarchical rule structure.

When evolving a hierarchy there are several groups of rules that work together to answer sets of elements. These are also referred to as niches, and because each niche caters to different elements they are not in direct competition with each other. This characteristic 
made it necessary to expand elitism to ensure that a variable number of rules can be copied to the next generation. This is done in cases where it is necessary that specific rules be copied in order to preserve the current level of confidence and support.

There are two types of elitism that are available to the genetic engine. The first type is the standard elitism, where the members with highest fitness are directly copied into the next generation. This step is done after all of the fitness values have been determined, but prior to selection of any members for the next generation.

\subsubsection{Standard elitism}

When the process starts it records the fitness for the best rule and uses this as a baseline for selecting other elite rules. The deviation percentage option dictates how much lower the fitness of another rule can be, and still considered elite. For example, if the best rule has a fitness of $81 \%$, then a $10 \%$ deviance would allow all rules with a fitness of $71 \%$ or above to be considered elite.

Particularly early in the life of the search, the set of rules has not had sufficient time to evolve, and therefore it is common to find very low fitness rules at the top of the hierarchy. When poor rules are in this position, and depending on the value for deviance, it means that almost the entire hierarchy can be considered elite. In order to prevent the entire population from being copied there is an upper bound parameter labeled the Max copy percentage. This value is typically low, perhaps $10 \%$, and stipulates the maximum percentage of the population that can be copied using elitism. This is necessary because 
preserving too much of the population will not allow new members to be created, which would likely curb the effectiveness of the evolution.

\subsubsection{Classification elitism}

The second type of elitism available for use in EvRFind is classification elitism. This approach has the same goal as standard elitism in that it preserves important members between generations. The difference between these two methods is defined by what are considered to be important traits in a particular rule.

Classification elitism does not make use of the standard fitness, but rather the result of applying the rule. To be considered elite, the rule must correctly answer at least one data element that has not been answered by a rule preceding it in the hierarchy. The rationale for this is that there is something unique about the rule, and this property is important enough that it should be preserved for future refinement.

At first glance it might seem that this form of elitism has a serious disadvantage, where very poor rules are copied into the next generation simply because they alone answer a particular element. Despite the fact that these rules are considered poor in general, it is important to determine what key features allow them to uniquely answer certain elements. These features can either be basic components for general cases, or specific logic that classifies outliers or other elements that go against the norm. 
Regardless of what is unique about each rule preserved, elitism guarantees that future generations will have a chance to extract what is important. If in a future generation these rules are evolved into better ones, then the original stepping-stone will be discarded, and the overall hierarchy will have improved.

Unlike the first version described, classification elitism has no upper bound on the number of rules that are copied. The justification for this is that no matter how small the confidence or support is, each unique feature must be fully explored. One foreseeable problem is that the possibility exists for every rule within the set to be copied without modification. In this case the population would remain static between generations and evolution would not occur, meaning that no improvement is possible.

Fortunately this problem is eliminated by EvRFind through the use of a flexible population size between generations. By temporarily increasing the maximum number of rules, the system introduces new terms and logic during each generation. This new genetic material is evaluated and given the opportunity to compete with existing rules, which allows the elite members to be replaced by improved versions of themselves.

To summarize, classification elitism is used for obtaining the highest possible data coverage when classifying elements into one of several predefined classes. This approach is not recommended for undirected mining tasks because the level of data coverage is of lesser importance when searching for interesting and obscure rules. The information being sought is usually a very small set of rules that describe a small subset within the 
domain, and although several areas within the dataset are explored in parallel, there is no guarantee of significant coverage.

\subsection{Fitness Proportional Selection}

After the elitism-copy process is completed, members of the current generation must be evolved and inserted into the population for the next generation. Rather than selecting solutions at random, which would not necessarily improve the quality of individuals in the next generation, the selection is performed with a bias towards the more fit members. Although not guaranteed, it is more likely that the members with a higher fitness will be selected, and those with poor fitness will be ignored and discarded.

The dilemma with fitness proportional selection is that because the more fit individuals are constantly being preferred, they are usually selected several times within a single generation. When this happens over several generations, a single individual with a high fitness tends dominate the entire population. This is a very bad scenario because all of the diversity in the population is lost. When this happens it means that future generations in the search can only improve through mutation, and this is a small probability when compared to using the crossover operation. This is also referred to as premature convergence because the members of the population have converged to a single answer before adequately exploring the search space. 
Although EvRFind uses fitness proportional selection, it is not subject to premature convergence because unlike standard GA, duplicate rules are not allowed to co-exist within the population.

After an individual has been selected for transfer to the next generation there is a probability that another genetic operation will occur. The first of these operations is genetic crossover.

\subsection{Crossover}

Crossover is a technique that is applied to two individuals in an attempt to generate at least one that is a superior combination of both. This requires two distinct individuals and a random point in each where the sequence of terms is split. The rules are broken and then recombined with those from the other rule, which creates two new members.

To illustrate this process, the two rules below were selected for crossover. An arbitrary point was chosen for each rule within the antecedent. In each of the rules below, the boldunderlined print indicates the first of the two sections as defined by the crossover-point.

$$
\begin{aligned}
& \text { 1: If } \underline{(\mathrm{A}<5)} \wedge(\mathrm{B}>10) \wedge(\mathrm{C}<=20) \text { Then }(\text { class }=1) \\
& \text { 2: If } \underline{(\mathrm{A}>0) \wedge(\mathrm{C}>2)} \wedge(\mathrm{D}<4) \text { Then }(\text { class }=2)
\end{aligned}
$$


When these two rules are crossed over, the following two rules are produced:

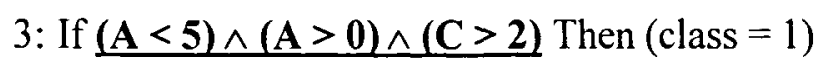

4: If $(B>10) \wedge(C<=20) \wedge(D<4)$ Then $($ class $=2)$

In EvRFind, this group of rules (1-4) is called a rule family. Exactly two of these rules, chosen according to the highest fitness, will be selected for the next generation. This differs from some GA implementations where crossover will indiscriminately select the child-rules (\#3 - \#4) and discard the parent-rules (\#1 - \#2).

One of the reasons for this difference is that standard GA systems do not consider the possibility that the child-rules are invalid. Because crossover operates at the term level, with the ability to change the size of rules, it is capable of creating rules that are not allowed based on restrictions set by the user during the configuration stage. When an invalid rule is detected after a crossover operation, it is removed from the family immediately.

The crossover operation does not necessarily generate all of the members in successive generations because it is only used on a probabilistic basis. In any event, whether generated by crossover or simply selected for its fitness, a rule is subject to mutation before being inserted into the next population. 


\subsection{Mutation}

Mutation is a technique that is used to randomly modify rules with the hope that some mutations will result in rules that are more fit than their original counterparts. In EvRFind there are two types of mutation, each with a range of possible values. The first type is called micro-mutation, and operates within the terms of each rule. The second is called macro-mutation, which does not affect the specifics of an individual term, but rather is used to add or remove terms from either side of the rule.

\subsubsection{Micro-mutation}

The goal of micro-mutation is to modify the terms in an attempt to add variation to the hierarchy. This type of mutation is capable of changing the term variables, constants, or the type of operation.

\section{Example}

If $(\mathrm{A}<5) \wedge(\mathrm{B}>10) \wedge(\mathrm{C}<=20)$ Then (class $=1)$

The probability that a micro-mutation will occur is uniform across all variables, constants, functions, and operators within all terms in the population. This operation does not consider terms or rules themselves, or anticipate the effect of the change.

In the example rule above, assume that the variable $B$ in the second term was arbitrarily selected for mutation. After the transformation it may become the variable $C$, as seen in the following rule: 
If $(\mathrm{A}<5) \wedge(\mathrm{C}>10) \wedge(\mathrm{C}<=20)$ Then (class $=1)$

Because the new rule is valid, and mutation is the last step in the evolutionary process, the rule above is copied into the next generation. If, however, the first term was changed rather than the second, the following rule might have been the result:

If $(B<5) \wedge(B>10) \wedge(C<=20)$ Then (class $=1)$

Because the variable $A$ in the first term was changed into the variable $B$, it results in a direct conflict with the second term. With two or more such opposing terms the rule is self-contradictory, and will be discarded immediately.

\subsubsection{Macro-mutation}

Macro-mutation is similar in that population members are modified and either moved into the next generation or discarded. However, the goal is to experiment with the specialization and generalization of existing rules.

Creating a more specialized version of a rule requires that the domain be further restricted by including additional constraints in the antecedent. The objective is to increase the accuracy by lowering the number of misclassified instances covered by the rule. The best result is an addition of terms that reduce the number of matches only by those elements that were previously being misclassified. 
To generalize a rule is the opposite of specialization, where constraints are removed in an attempt to increase coverage. In the best case the constraints are relaxed enough to answer more elements, but without incurring the penalty of reduced accuracy.

\section{Example}

If $(\mathrm{A}<5) \wedge(\mathrm{B}>10) \wedge(\mathrm{C}<=20)$ Then $($ class $=1) \quad$ [Original rule]

If $(\mathrm{A}<5) \wedge(\mathrm{B}>10) \wedge(\mathrm{B}<20) \wedge(\mathrm{C}<=20)$ Then $($ class $=1) \quad$ [Specialization]

If $(\mathrm{A}<5) \wedge(\mathrm{C}<=20)$ Then (class $=1) \quad$ [Generalization]

Both mutation types are used together during the evolutionary process, and can be controlled using varying probabilities.

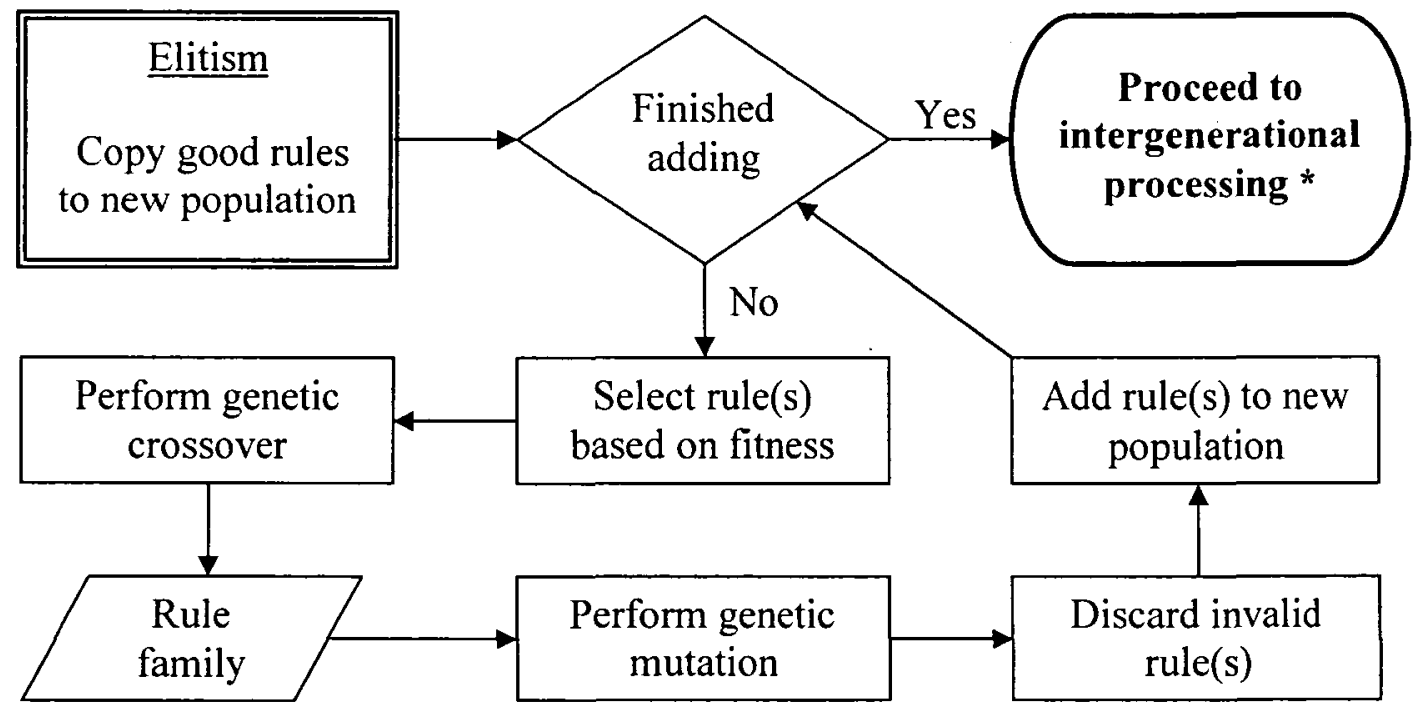

Figure 5.4: Creating a new population 
This chart is the continuation of a previous chart, the Single experiment overview, on page 99 .

$\left({ }^{*}\right)$ : The process labeled "Proceed to intergenerational processing" expands and is presented as a separate diagram that contain details specific to that process.

Flowchart expansion reference

Intergenerational processing - Page 112

\subsection{Intergenerational Processing}

Evolutionary systems, such as GA and GP, evolve populations from one generation to the next by using a variety of techniques. In ways that are similar to those described previously in this chapter, these techniques are normally restricted to genetic operations that are based on Darwin's theory of evolution. Although this process has had success in many applications, EvRFind does not rely solely on evolution.

In an attempt to maximize search efficiency and overall performance, EvRFind performs a number of non-evolutionary functions between generations. This stage is called Intergenerational Processing, and there are several subcomponents that are used during this part of the search. 


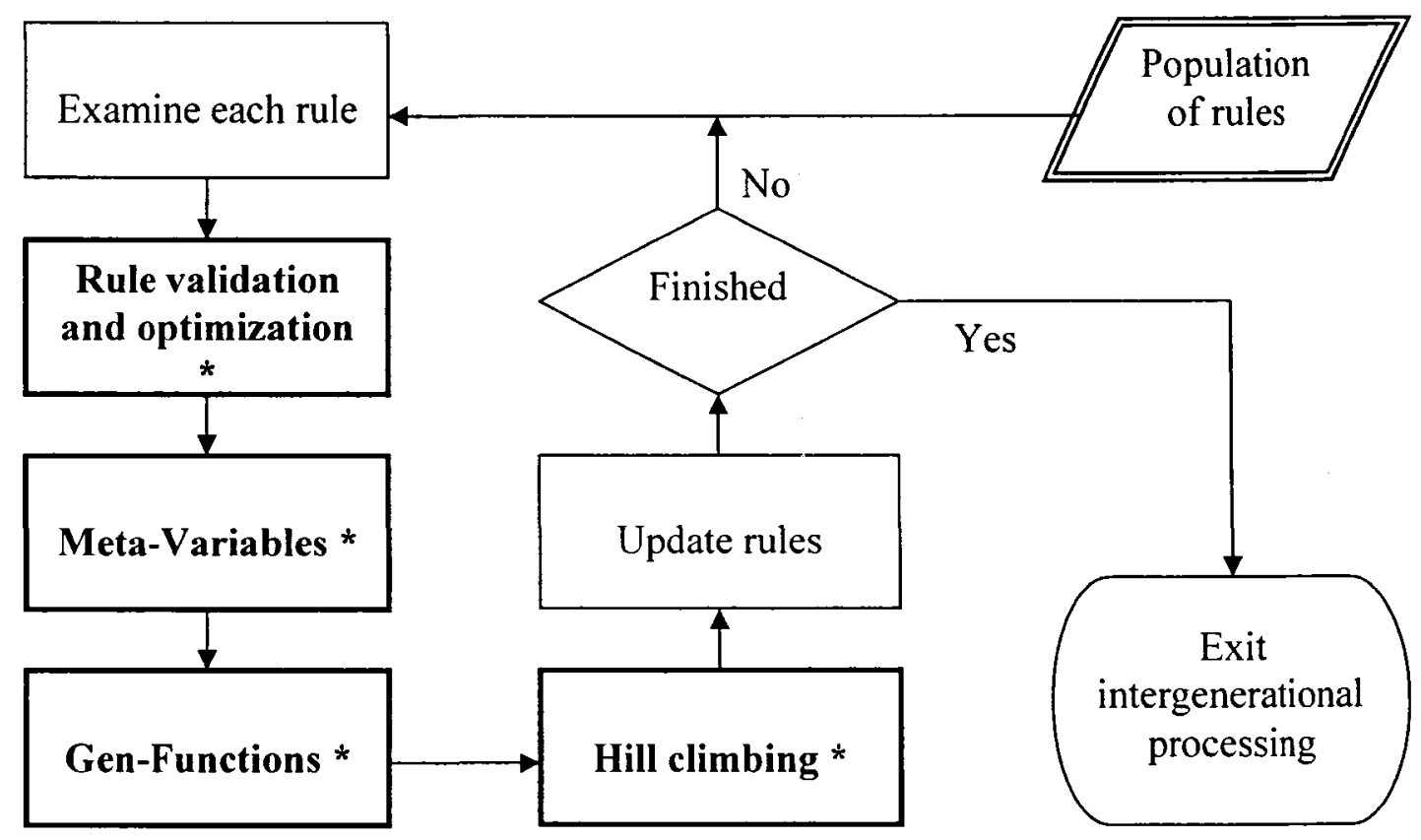

Figure 5.5: Intergenerational processing overview

This chart is the continuation of a previous chart, Creating a new population, found on page 110 .

$(*)$ : The processes labeled "Rule validation and optimization", "Meta-Variables", "GenFunctions", and "Hill climbing" each expand and are presented as separate diagrams that contain details specific to that process.

Flowchart expansion reference

Rule validation and optimization - Page 118

Meta-Variables - Page 124

Gen-Functions - Page 129

Hill-Climbing - Page 148 


\subsubsection{Rule Validation and Optimization}

Due to the fact that the genetic operations behave somewhat randomly it is often the case that rules are modified such that they become invalid. EvRFind handles this situation during the intergenerational processing stage by using a non-evolutionary approach to modify and replace rules. This is intended to preserve the validity and efficiency of each rule.

Mechanisms of this type are not typically used in Genetic Algorithms. However, the idea is similar in some respects to a Genetic Programming variant known as Typed-GP [Montana, 1995]. Typed-GP guarantees that evolved programs will not contain critical errors, thus saving processing time by eliminating worthless members from the population.

A popular argument against using this type of mechanism is that it is cheating, where to cheat means that forces other than natural selection have control over at least part of the population. The first reason for taking this position is that, in theory, the selection pressure will eliminate worthless members without interference. Secondly, by eliminating individuals that are not selected against naturally, there is a risk that useful genetic material is being lost as a consequence. Regardless of these points, EvRFind allows the user to specify one or more options that override the natural selection process.

The genetic engine in EvRFind makes these screening options available because of the results from many experiments that were performed during the research and evaluation 
stage. It was determined that by allowing the system to remove certain types of rules, not only was there an improvement in the time required to complete the search, but the rule sets performed better on average.

A good example of the gain offered by using this option was observed during the tenth generation of a classification experiment. Using all of the same data and parameters, except for the intergenerational processing options, two searches were run in parallel. By the tenth generation in the system that allowed redundancy and contradictions to exist, there were very few valid rules left in the population. In the competing system, where screening for invalid and ineffectual rules was being performed, many of the rules were sound and remained in place until the end of the search. This same scenario was observed in many experiments and the option was eventually adopted as the norm.

In order to prevent the loss of diversity expected with the non-evolutionary removal of rules, each deleted member is immediately replaced. This replacement is done through the use of rule modification or regeneration.

When a rule is deemed invalid then the first step is to modify it such that it becomes valid. The technique used for this is the systematic deletion of terms from antecedent, consequent, or both. If this is not possible because of the rule structure, or an excess of similar members in the population, then a complete substitution will be made. If after replacing or modifying the rule the result is still invalid, or is a duplicate with another rule in the set, this technique is continually applied until all conditions are satisfied. 
EvRFind supports two options that cover rules in need of optimization or correction: elimination of extra numeric restrictions and elimination of conflicting restrictions.

\subsubsection{Elimination of extra numeric restrictions}

Rules that can potentially benefit from this option include those with redundant terms as well as rules that result in a logical tautology. Rules with these types of properties are not actually invalid, but they may be longer than necessary or even completely useless. In some cases this also erodes the confidence of the rule, although typically the major disadvantage is a loss of support. Some example rules that fall into this category are as follows:

1: If $(\mathrm{A}<5) \wedge(\mathrm{B}<10) \wedge(\mathrm{A}<10)$ Then $($ class $=4)$

2: If $(\mathrm{A}<5) \wedge(\mathrm{B}<10)$ Then $(\mathrm{X}=4) \wedge(\mathrm{A}<10)$

3: If C (exists) Then C (exists)

As will be shown in the next step, both rules $\# 1$ and $\# 2$ can be modified such that they become valid and more efficient. Rule \#3 is a simple tautology that will be automatically removed and replaced with a new rule.

Once a rule has been targeted for modification or removal the system checks another user specified setting that biases the system towards removing terms following a particular theme. The two choices are eliminating more specific rules, and eliminating more general 
rules. This choice can also be varied and will have different effects depending on the dataset.

In the first rule above there are two restrictions placed on the variable $A$, making one of them pointless. The decision of which to remove is described by the following logic:

A) Elimination removes more specific rules. EvRFind checks the variable type, valid ranges, and comparison operations for each rule to determine which variation is more specific. In this example we assume that the types are integers and the valid range is 0-25.

After removing the more specific terms, the new rules to replace \#1 and \#2 are:

1: If $(\mathrm{B}<10) \wedge(\mathrm{A}<10)$ then $($ class $=4)$

2: If $(B<10)$ then $(X=4) \wedge(A>10)$

In both of these cases it was determined that $(A<5)$ term was the more restrictive, and hence the more specific term. It was removed from the antecedent of both rules.

B) Elimination removes more general rules. This has exactly the opposite effect as the previous option and the $(\mathrm{A}<10)$ term is removed from the two rules as follows: 
1: If $(\mathrm{A}<5) \wedge(\mathrm{B}<10)$ Then $($ class $=4)$

2: If $(\mathrm{A}<5) \wedge(\mathrm{B}<10)$ Then $(\mathrm{X}=4)$

Considering the valid integer ranges, these rules are obviously more restrictive than the set derived using the first option.

\subsubsection{Elimination of conflicting restrictions}

Rules that fall into this category are invalid because they are self-contradictory. These rules are more detrimental to the entire set because their confidence is always $0 \%$, and they take space in the population as well as consume system resource without benefit. Following are some examples of this type of rule:

1: If $(\mathrm{A}<5) \wedge(\mathrm{B}<10) \wedge(\mathrm{A}>10)$ then $($ class $=4)$

2: If $(\mathrm{A}<5) \wedge(\mathrm{B}<10)$ then $(\mathrm{X}=4) \wedge(\mathrm{A}>10)$

3: If C (exists) Then $\sim \mathrm{C}$ (does not exist)

As with the example for the previous option, both rules $\# 1$ and $\# 2$ can be modified such that they become valid. Rule \#3 is a simple contradiction that will be automatically removed and replaced with a new rule.

In each of rules \#1 and \#2 the variable $A$ is used within more than one term. Although this condition alone does not invalidate a particular rule, the use of it as shown in both examples does. Specifically, the first rule will never be executed because $A$ cannot be 
both less than 5 and greater than 10 . The second rule is a contradiction because it states that $A$ will be greater than 10 if it is first less than 5 .

How EvRFind modifies the rules is also dependent on the option directing the removal of more specific or more general terms. In the case that the user selects more specific terms, $(A<5)$ will be deleted. In the opposite case, where the user selects more general terms, $(A>10)$ will be deleted. Once again, these examples only hold true depending on the allowed range for variable $A$.

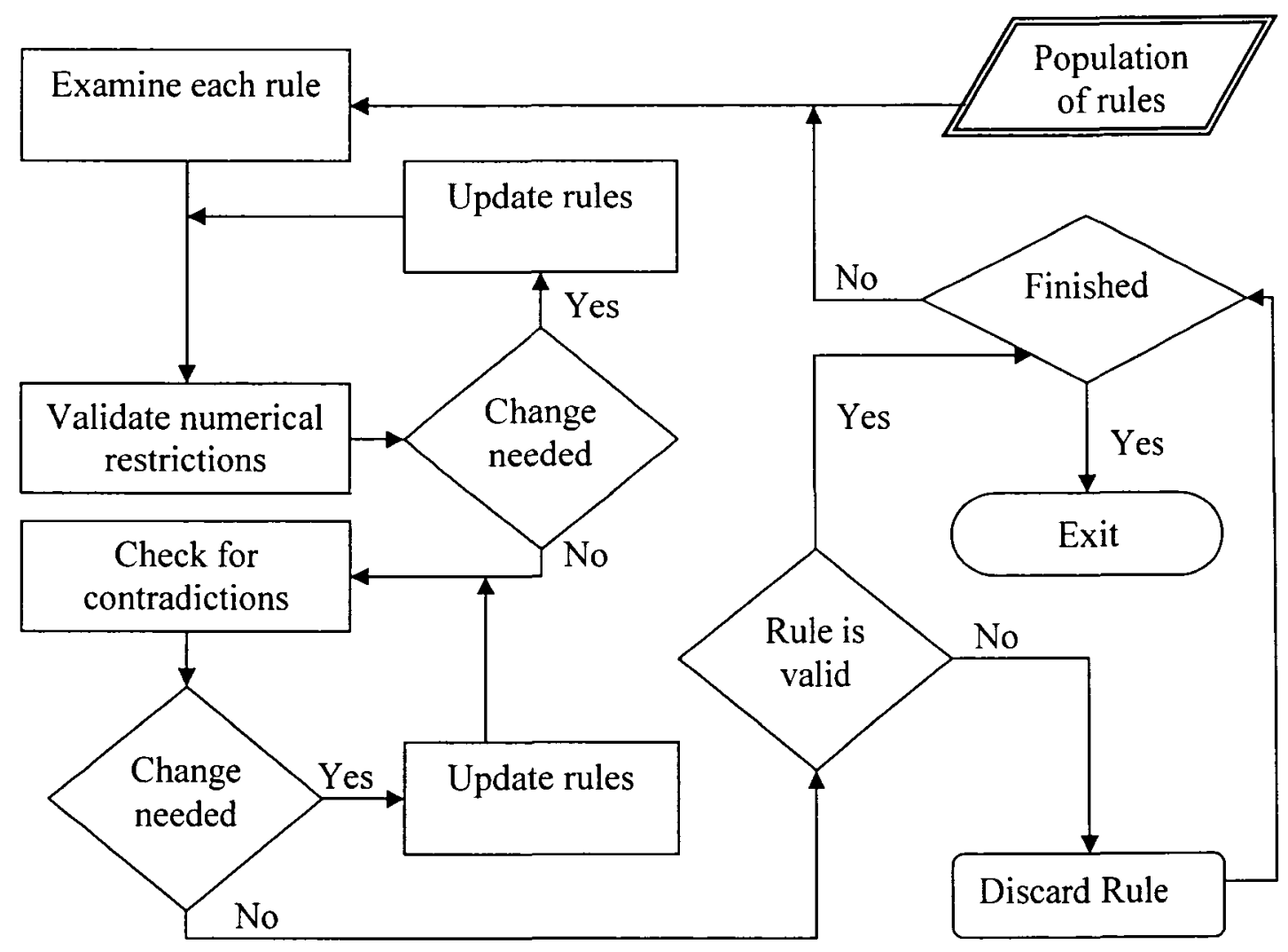

Figure 5.6: Rule validation and optimization overview

This chart is the continuation of a previous chart, the Intergenerational processing overview, on page 112 . 


\subsubsection{Meta-Variables}

\subsubsection{Description}

One of the major components in the intergenerational section is a language extending term called the Meta-Variable. Meta-variables are a form of constructive induction, which was described in Chapter 4.2: Constructive Induction, starting on page 63. These are aggregate variables that represent specific terms, or conjunctions of terms, in a rule. The result is a Boolean evaluation that indicates true or false when evaluating the rule against an example instance.

The theoretical benefit behind creating Meta-Variables is that the grammar becomes increasingly more descriptive by adding building blocks discovered in earlier generations. As will be seen in an example below, Meta-Variables are only created using building blocks that are considered to be good in the current generation of rules. The result is an atomic unit that is immune to disruption caused by genetic operations, and will persist as part of the grammar as long as it is useful.

Another benefit realized by creating complex elements of the language is shorter rule length. In some cases this makes a rule easier to read, but comprehensibility is not the only goal. In practice there is normally a limit to the length of each rule. This length can be measured by the number of terms in the antecedent, or consequent, or both. By reducing the list from several terms into one, it means that more terms can be appended and still adhere to the size restriction. 
The simple act of combining terms in order to circumvent a length restriction would go completely against the reason that a size cap exists in the first place. In the case of MetaVariables, however, the terms used to create the aggregates have already been recognized as having value. If several aggregates of good terms are used within a rule, and semantically the size limit is surpassed, then it is acceptable because the expansion was limited to sufficiently good combinations of terms.

The reason that Meta-Variables are guaranteed to have value is the selection process used when they are being created. As will be seen in the next example, Meta-Variables are created using a group of candidate rules that are chosen because they are $100 \%$ confident. Although this criterion does not take into consideration the number of instances potentially covered by the new variable, it does encourage accuracy.

Once a Meta-Variable is created there is no process of review to determine whether it remains useful in successive generations. As will be more completely described below, it is not necessary to have a process that breaks apart poorly performing Meta-Variables to restore the basic components. If a Meta-Variable performs poorly in future generations then EvRFind will simply not make use of it.

\subsubsection{Syntax}

Each Meta-Variable is internally represented by a conjunction of terms. It is identified within a rule using an alphabetic identifier that is prefixed with the pound sign (“\#”). 
Meta-Variable \#A : $=\left[\left(X_{1}\right) \wedge \ldots \wedge\left(X_{n}\right)\right],\{n>=1\}$

- $X$ denotes a term as previously defined in section 5.1.2: Terms, starting on page 70.

Each Meta-Variable is used in place of a term within a rule, and evaluates to true or false.

\subsubsection{Example}

In the following example, all of the rules are $100 \%$ confident. They were chosen as candidates from the population in a single generation because of their accuracy on the training set.

\section{Candidate rules}

If $(A=5) \wedge(B>D) \wedge(C=30)$ then $($ Type $=2)$

If $(A=5) \wedge(C<15) \wedge(B>D)$ then $($ Type $=3)$

If $(A=5) \wedge(B>D) \wedge(C>30)$ then $($ Type $=1)$

When EvRFind examines these rules it finds that the most commonly occurring combination of terms is: $(\mathbf{A}=\mathbf{5})$ and $(\mathbf{B}>\mathbf{D})$. Because this configuration appears to be a key component in several rules, without taking into consideration that the consequents are different, EvRFind creates a Meta-Variable that represents the conjunction of these comparative terms. The new variable is denoted as \#A below, and should not be confused with the original variable labeled $\mathbf{A}$. 


\section{After replacement}

If $(\# A) \wedge(C=30)$ then $($ Type $=2)$

If $(\# A) \wedge(C<15)$ then $($ Type $=3)$

If $(\# A) \wedge(C>30)$ then $($ Type $=1)$

The technique is similar to chunking, which has been described in [Newell, 1990] as the primary mechanism for learning from experience. The aggregate functions become building blocks that extend the representation language. Since they are extensions of the vocabulary, they are protected from disruption by crossover, and only need to be discovered once.

\subsubsection{Candidate selection and replacement}

When rules are selected as Meta-Variable candidates from the population, the restriction of $100 \%$ confidence exists for two main reasons. First, as mentioned previously, it is important to limit extensions to the grammar to components that are likely to be accurate. If this were not the case then a large number of poor language extensions could exist, and this could have a negative effect on the search.

Secondly, the confidence restriction is enforced to ensure that only a sufficiently small subset of the population is examined. This is necessary because the search for common terms within a group of rules is a computationally expensive operation, and using it on a large number of rules will slow down the search dramatically. 
After a Meta-Variable is created it needs to be inserted into the population. Originally, all of the rules in the entire population were examined. If the terms used to create the variable were found then they would be replaced. Although this seemed like a good idea, it was slow to perform a search-and-replace on the entire population, so performance evaluation was done by replacing only the terms in rules belonging to the candidate set.

Unexpectedly, the result of replacing terms only within the candidate set showed a marked improvement in predictive accuracy. Not considering computational effort, but rather the number of generations, better rules were consistently discovered faster when not performing a global replacement. Further analysis of this showed that global replacement had a negative impact for at least one reason, which was the premature removal of useful terms.

The problem stemmed from the fact that a conjunction of terms was good in some cases, but this does not give credit to work done prior to bringing these good terms together. Not taken into consideration was the fact that good terms were put into the same rule because they were also good on their own, and with global replacement it removed many of these from the population. Without sufficient good terms to draw on, the crossover recombination was not producing the same number of superior rules, and the evolution suffered overall. If global replacement were going to be used in the future then some kind of mechanism to break apart Meta-Variables, into their basic components, would have to be implemented into order to prevent this problem. 
Further experimentation lead to the discovery that the widespread creation and usage of Meta-Variables too early in the search lead to poor results. This becomes more evident with each generation because older Meta-Variables are typically inferior when compared to those that are created using candidate rules that have survived through several generations of evolution. For this reason, at most, only a single Meta-Variable can be created during each generation.

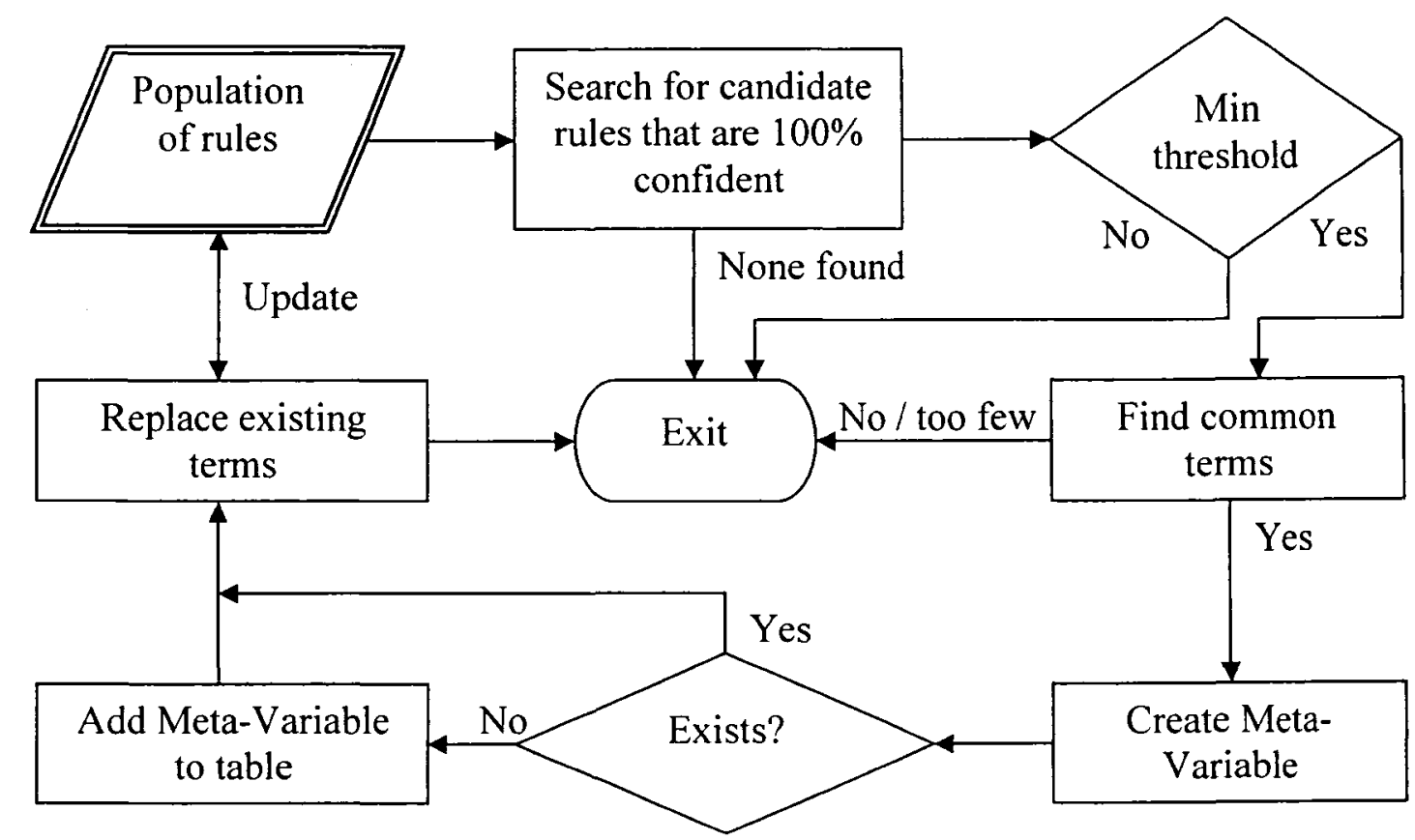

Figure 5.7: Meta-Variable creation

This chart is the continuation of a previous chart, the Intergenerational processing overview, on page 112 . 


\subsubsection{Post discovery usage}

After a Meta-Variable is created and added to the language it is available for use by the rule creation and modification routines. A Meta-Variable can be included in any rule that is receiving a new term or where an existing one is being modified. Following from this, if a Meta-Variable is one of several common terms found within a subset of $100 \%$ confident rules, then the creation of a new Meta-Variable that references an existing one is possible.

Consider the following Meta-Variables that were discovered at different times over the course of several generations:

MV: \#A [(wait $>=25) \wedge($ wait $<=50)]$

MV: \#B [(delay < wait) $]$

$\mathrm{MV}: \# \mathrm{C}[(\# \mathrm{~A}) \wedge($ available_time $>$ delay $)]$

$\mathrm{MV}: \# \mathrm{D}[(\# \mathrm{~B}) \wedge(\# \mathrm{C})]$

Each of these Meta-Variables, \#A - \#D, represents a concept that was popular enough to be added to the language. As seen in the third and fourth examples, higher level constructs can be created using other language extensions, and this can continue as long as the conditions are met.

The following two rules accompany these Meta-Variables in a predictive model: 
If $($ sound $=4) \wedge(\# D)$ then $($ CLASS $=5)$

If $($ sound $=2) \wedge(\# D)$ then $($ CLASS $=5)$

These two rules do not entirely cover class 5 , but having unclassified instances is not as important as the point that without \#D, the rules would have to be put together using a larger number of terms, such as:

If $($ wait $>=25) \wedge($ sound $=4) \wedge($ available_time $>$ delay $) \wedge($ delay $<$ wait $) \wedge($ wait $<=50)$ then $($ CLASS $=5)$

If $($ sound $=2) \wedge($ wait $<=50) \wedge($ available_time $>$ delay $) \wedge($ wait $>=25) \wedge($ delay $<$ wait $)$ then $($ CLASS $=5)$

In examining the two rules it is easy to see that the only difference is a single mutation in the constant value being compared to the sound attribute. When dealing with fewer terms, as in the case where the Meta-Variable is used, it is more likely that this mutation will occur. The terms that are protected by $\# \mathbf{D}$ are not subject to mutation. Following this, it can be inferred that the use of building blocks speeds the evolutionary search by focusing mutation on untested parts of the rule.

During experiments on the poker hand dataset, the beneficial effect of having MetaVariables within a rule that was being mutated was seen. For specific details, refer to Chapter 6.7.4.4: Concept of a flush, starting on page 208. 


\subsubsection{Gen-Functions}

The next component in the intergenerational section is another language-extending concept called the Gen-Function. This is also a rule generalization technique, and it is used to abstract higher level rules from groups of related but more specific rules.

As in the case of the Meta-Variable, the Gen-Function is another type of constructive induction. This concept was described in Chapter 4.2: Constructive Induction, starting on page 63.

\subsubsection{Description}

The Gen-Function component was designed to examine and generalize sets of rules based on functions contained within a library. These functions highlight common themes between rules, and these themes can be used to group rules together into more general concepts. Examples of groupings include transitivity of equality, linear relationships between attributes, and related results for mathematical or statistical operations.

Gen-Functions are similar to new attributes derived through attribute construction in AQ17-DCI learning [Bloedorn, Michalski, 1996], where the language is extended based on regularities discovered within the training examples. Their technique, however, relies heavily on exhaustive testing in order to generate new attributes. Without a search space reduction task running beforehand, such as the way that EvRFind uses evolution to guide the search, this process is computationally too expensive to evaluate a large number of 
functions. In the case of AQ17-DCI, the user is required to specify which functions should be considered for attribute construction.

The disadvantage to requiring input from the user is that it constricts the boundaries of the search to what the user considers to be reasonable. This added degree of supervision has the potential to exclude useful functions, and in turn, some useful attributes might not be discovered. An example of where useful results are discovered through the use of a non-intuitive function can be seen in Chapter 6.7.4.12: Concept of royal flush - Revisited, starting on page 221 .

The goal of generalization in EvRFind is to build rules that are more accurate, more comprehensible, and have greater coverage than larger groups of rules describing the same concept. With a large library of functions that do not have to be selected for each domain, the language will automatically evolve as much as possible in order to accurately classify all examples. 


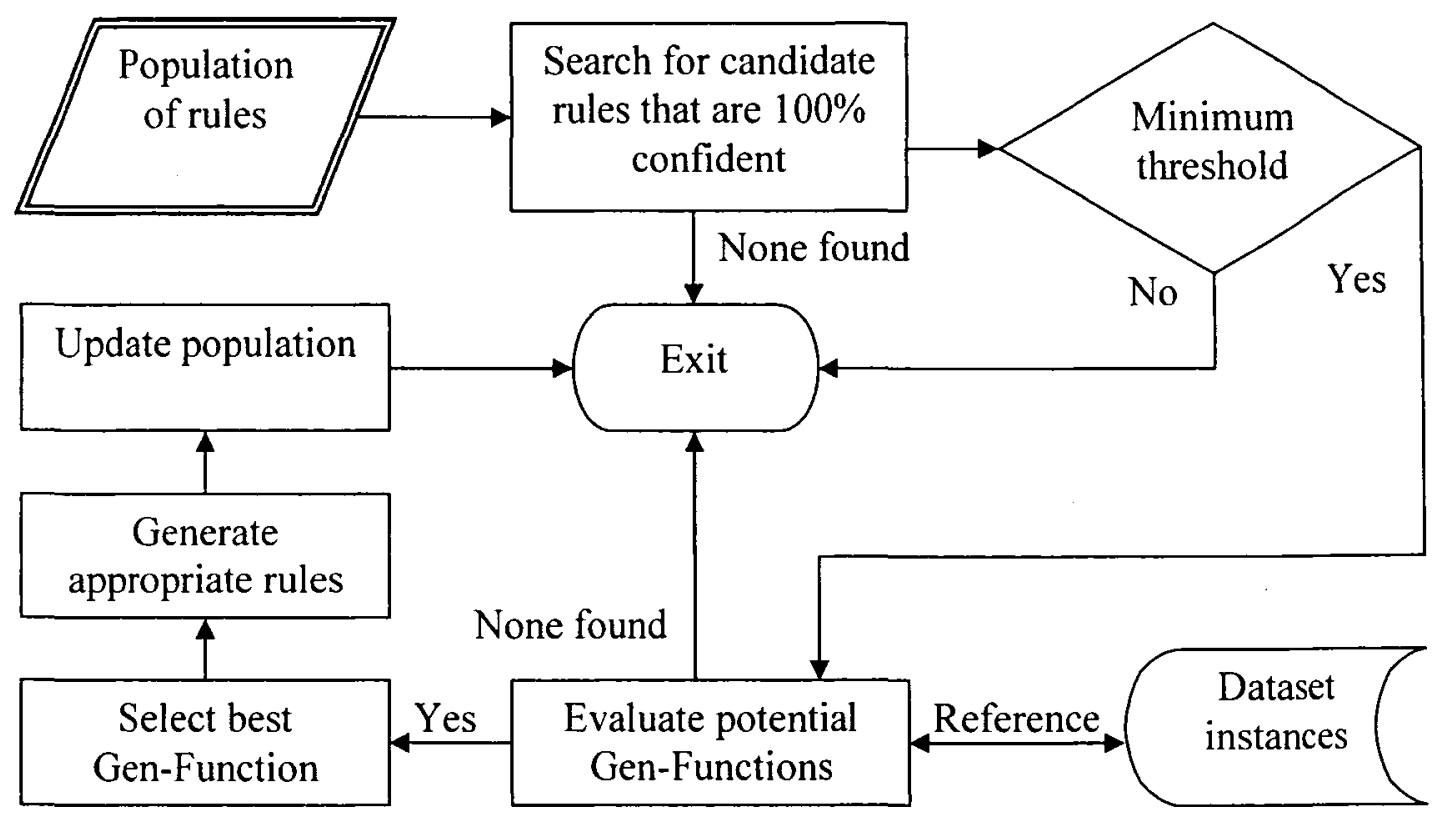

Figure 5.8: Gen-Function creation

This chart is the continuation of a previous chart, the Intergenerational processing overview, on page 112 .

\subsubsection{Syntax}

Each Gen-Function is internally represented by a primitive function, and optionally, several parameters. It is identified within a rule using an alphabetic identifier that is prefixed with the dollar sign ("\$").

Gen-Function $\$ A:=\left[\right.$ Primitive $($ class $\left.\left.=C), P_{1}, \ldots, P_{n}\right)\right]$

- $C$ optionally specifies what valid attribute-class for which this can be used 
- Primitive refers to the base function. A list of primitives available at the time of the writing can be seen later under section 5.11.3.7: List of Implemented GenFunctions, starting on page 145.

- $P$ represents a parameter, of which some may be optional, that are specific to the primitive function.

Each Gen-Function is used within a term and returns a value that is specific to the primitive function.

\subsubsection{Example}

An example of rule generalization can be seen in the following example. The defining rules for this dataset are simple:

1) There are six binary predictive attributes with possible values $\{0,1\}$.

2) There is one class attribute with possible values $\{0,1,2,3,4,5,6\}$.

3) The class attribute is equal to the number of $1 \mathrm{~s}$ in the set of predictive attributes.

4) The dataset is the complete set of $2^{6}$ records, with one example for every possible combination of attribute values, and is used for both training and evaluation.

\section{Candidate rules}

If $(B=1) \wedge(C=0) \wedge(D=0) \wedge(E=0) \wedge(F=0)$ then (Class $=1)$

If $(A=0) \wedge(B=0) \wedge(D=1) \wedge(E=0) \wedge(F=0)$ then (Class $=1)$ 
These two example rules classify a subset of the data as a class 1 . Notice that these rules are not $100 \%$ confident over all possible instances. Given that each rule only specifies five terms, they do not exclude all of the cases where the class is 2 . However, the addition of new terms to achieve perfect accuracy is not necessary to see the benefits of generalization.

A simple examination of these rules reveals that they are very specific in terms of attributes and their values. In most datasets, a situation such as this would require a large number of rules to fully describe the concept. In this example it would require six rules to fully describe class 1 . Each rule would have to explicitly state the value of each term:

If $(\underline{A=1}) \wedge(B=0) \wedge(C=0) \wedge(D=0) \wedge(E=0) \wedge(F=0)$ then $($ Class $=1)$
$\ldots$
If $(A=0) \wedge(B=0) \wedge(C=0) \wedge(D=0) \wedge(E=0) \wedge(\mathrm{F}=1)$ then $($ Class $=1)$

Rather than discover these more numerous and more complex rules, during the intergenerational processing stage the Gen-Function component notices the similarity and attempts to generalize them. This is not done exhaustively because the operation is computationally expensive. Instead, EvRFind relies on evolution to choose the subsets that warrant further examination. After experimenting with a number of library functions it can result in an entire group of rules being replaced. 


\section{$\underline{\text { After replacement }}$}

1: GF: \$A $[$ NEV $($ class $=$ General_Antecedent $)=5$ Constant $=0\}]$

2: If $(\$ A=1)$ then $($ Class $=1)$

Line 1 is the definition of a Gen-Function. It relies on the built-in function "NEV (number of equal values)", which accepts three parameters in this example. The parameters are:

a) class = General_Antecedent; This means that the function assumes the scope of all predictive attributes.

b) $=5$; This specifies that there must be exactly five equal values for this function to evaluate to true.

c) Constant $=0$; This specifies that the five equal values cannot be arbitrary. Each of the five values must be equal to zero.

Line 2 is the rule that makes use of the new Gen-Function, \$A. It states that if there is exactly one occurrence of $\mathbf{\$ A}$ within the example instance being evaluated then the class is 1 .

Full translation: "Within the subset of attributes that are specified to be of class 'General_Antecedent', if there are exactly five values that are equivalent to each other, and all five of them are the constant value ' 0 ', and this occurs exactly once within the instance, then the example is classified as 'class $=1$ ' (contains one ' 1 ')." 
The Gen-Function and corresponding rule are general enough to fully classify every example that contains only a single ' $l$ ', yet specific enough such that no instances from other classes will be misclassified.

\subsubsection{Candidate selection}

Gen-Functions are created when common themes are found in a set of discovered rules. As in the case with Meta-Variables, EvRFind relies on evolution to select candidate rules, and another process examines the candidates for regularities.

Once a group of candidate rules is selected, the next step depends on the Gen-Function being evaluated. In some cases the rules will be studied, while in other cases the corresponding data elements will be investigated.

\section{Example: Gen-Functions created using rules alone}

An example of Gen-Function creation using the rules as a guide is the Number of Equal Values (NEV) function. Consider the knowledge in the following rules:

1: If $(\mathrm{C} 3=\mathrm{C} 1) \wedge(\mathrm{C} 1=\mathrm{C} 4)$ then $($ CLASS $=2)$

2: If $(\mathrm{C} 2=\mathrm{C} 1) \wedge(\mathrm{C} 2=\mathrm{C} 4)$ then $($ CLASS $=3)$

3: If $(\mathrm{C} 1=\mathrm{C} 4) \wedge(\mathrm{C} 2=\mathrm{C} 4)$ then $(\mathrm{CLASS}=3)$

4: If $(\mathrm{C} 1=\mathrm{C} 4) \wedge(\mathrm{C} 4=8) \wedge(\mathrm{C} 4=\mathrm{C} 2)$ then $(\mathrm{CLASS}=3)$

5: If $(\mathrm{C} 2=\mathrm{C} 5) \wedge(\mathrm{C} 3=\mathrm{C} 4) \wedge(\mathrm{C} 4=2)$ then $(\mathrm{CLASS}=4)$ 
6: If $(\mathrm{C} 2=\mathrm{C} 1) \wedge(\mathrm{C} 3=1)$ then $(\mathrm{CLASS}=4)$

7: If $(\mathrm{C} 2=\mathrm{C} 1) \wedge(\mathrm{C} 3=5)$ then $(\mathrm{CLASS}=5)$

8: If $(\mathrm{C} 2=8) \wedge(\mathrm{C} 3=8) \wedge(\mathrm{C} 4=1) \wedge(\mathrm{C} 5=1)$ then $(\mathrm{CLASS}=9)$

The important points in these eight rules are:

- Rules \#1 - \#3 have three common values, none with specific constants.

- Rule \#1 has a different consequent than \#2 - \#3.

- Rule \#4 has three common values, and they are all equal to the value 8 .

- Rule \#5 has two non-specific common values $(\mathrm{C} 2=\mathrm{C} 5)$, and two common values with a specific constant $(\mathrm{C} 3=\mathrm{C} 4=2)$.

- Rule \#6 has one set of common values and a non-overlapping constant. The first set is variable and the second set has a specific constant.

- Rule \#7 is similar to rule \#6 except that the constant value is different, which results in a different consequent.

- Rule \#8 has two sets of two common values, the first set with the constant value 8 and the second set with the constant value 1 .

When EvRFind examines the rules and finds these details, the algorithm creates a list of tentative Gen-Functions. These functions, as well as more general versions of them, are temporarily created and evaluated. If EvRFind re-creates a function that already exists then it will be removed from the list before any evaluation is performed. The temporary 
list is used to assess which, if any, Gen-Functions would be well-suited to the current dataset. The intermediate list for this example is as follows:

GF \#1: $[$ NEV $($ class $=$ General_Antecedent $)=3\{$ Variable $\}]-$ Count: 3

GF \#2: $[\mathrm{NEV}($ class $=$ General_Antecedent $)=3\{$ Generalized $\}]-$ Count: 4

GF \#3: [NEV $($ class $=$ General_Antecedent $)=3\{$ Constant $=8\}]-$ Count: 1

GF \#4: $[$ NEV $($ class $=$ General_Antecedent $)=2$ \{Variable $\}]-$ Count: 3

GF \#5: $[$ NEV (class $=$ General_Antecedent $)=2\{$ Generalized $\}]-$ Count: 6

GF \#6: [NEV(class $=$ General_Antecedent $)=2\{$ Constant $=2\}]-$ Count: 1

GF \#7: $[N E V($ class $=$ General_Antecedent $)=2\{$ Constant $=8\}]-$ Count: 1

GF \#8: $[$ NEV (class $=$ General_Antecedent $)=2\{$ Constant $=1\}]-$ Count: 1

- GF \#1 covers three instances found in rules \#1 - \#3.

- GF \#2 is a Generalized version of GF \#1, which means that it is valid whether the relationship is known to have a specific value or not. It covers the same instances as $\mathrm{GF} \# 1$, and with the Variable restriction removed it also covers rule \#4.

- GF \#3 covers only rule \#4, where the constant value is specified.

- GF \#4 covers the equal value pairs, without constants specified, from rules \#5, \#6, and \#7.

- GF \#5 is a Generalized version of GF \#4. It covers those pairs that GF \#4 covers, as well as pairs for which constants are specified. There is one in rule \#5, and two in rule \#8.

- GF \#6 covers the pair from rule \#5. 
- GF \#7 covers one of the two pairs from rule $\# 8$.

- GF \#8 covers the pair from rule \#8 that wasn't covered by GF \#7.

The intermediate Gen-Function table in this example shows eight potential functions. Each one has a counter that indicates how many of the candidate rules could be at least partly replaced by the function. In most cases the generalized version of a particular function will be the best choice because it brackets together different constant values. Sometimes, however, a specific constant value is required to properly classify a given example. For this reason, EvRFind does not automatically discard potential GenFunctions when creating the generalized version. The Gen-Function with the highest count is selected for installation in the population. If several Gen-Functions have the same count, then all of them will be added during the same generation.

Another value that is stored with each potential Gen-Function is the result from each evaluation. For example:

GF $\$ A:[N E V($ class $=$ General_Antecedent $)=2\{$ Generalized $\}]$

1: If $(\mathrm{D} 1=\mathrm{D} 2) \wedge(\mathrm{D} 3=\mathrm{D} 4)$ then $(\ldots)$

2: If $(\mathrm{D} 5=\mathrm{D} 2)$ then $(\ldots)$

The result of evaluating $\$ A$ on each of these two rules is different.

- $(\$ A=2)$ is true for the first rule, but false for the second rule

- $(\$ A=1)$ is true for the second rule, but false for the first rule 
All of the evaluation results, determined from different rules, are recorded and will be referenced if the Gen-Function is added to the population.

\section{Example: Gen-Functions created using rules and data}

An example of Gen-Function creation using both candidate rules and the example instances that they cover is the Range of Values in Class (RGC) function.

Depending on the Gen-Function being considered there are certain attributes that will be ignored. For example, it is assumed that if an attribute is not comparable by at least one of the " $<,<=,>,>=$ " operators then it is a nominal value. Functions such as Minimum Value, Maximum Value, Average Value, and the Value Range would not make sense when used to describe nominal values. If all of the values in the dataset are nominal then these Gen-Functions will not be created.

When assessing the worth of functions such as RGC it requires that the data be examined, as is the case with AQ17-DCI [Bloedorn, Michalski, 1996]. Because examining all of the data would require too much computational effort, EvRFind partitions the data into subsets. This can be done in many ways including clustering and other unsupervised learning methods, but EvRFind partitions the examples by looking at which rules accurately cover them.

Consider the knowledge in the following rules from the previous example: 
1: If $(\mathrm{C} 3=\mathrm{C} 1) \wedge(\mathrm{Cl}=\mathrm{C} 4)$ then $(\mathrm{CLASS}=2)$

2: If $(\mathrm{C} 2=\mathrm{C} 1) \wedge(\mathrm{C} 2=\mathrm{C} 4)$ then $(\mathrm{CLASS}=3)$

3: If $(\mathrm{Cl}=\mathrm{C} 4) \wedge(\mathrm{C} 2=\mathrm{C} 4)$ then $(\mathrm{CLASS}=3)$

The instances covered by the three rules above are not displayed here, but EvRFind maintains a list of them with other information for each generation. For functions such as RGC, MNC (Minimum Value in Class), and MXC (Maximum Value in Class), the example instances for every candidate rule are put into a temporary list. This list is used by the Gen-Function evaluation component to evaluate the worth of potential functions.

Take it as fact that the following instances are covered by the three rules above:

\begin{tabular}{|c|l|c|c|c|}
\hline Rule & Data & RGC & MNC & MXC \\
\hline 1 & $\mathrm{Cl}=5, \mathrm{C} 2=6, \mathrm{C} 3=5, \mathrm{C} 4=5, \mathrm{C} 5=9, \mathrm{CLASS}=2$ & 5 & 5 & 9 \\
\hline 1 & $\mathrm{C} 1=2, \mathrm{C} 2=6, \mathrm{C} 3=2, \mathrm{C} 4=2, \mathrm{C} 5=2, \mathrm{CLASS}=2$ & 5 & 2 & 6 \\
\hline 1 & $\mathrm{C} 1=5, \mathrm{C} 2=1, \mathrm{C} 3=5, \mathrm{C} 4=5, \mathrm{C} 5=3, \mathrm{CLASS}=2$ & 5 & 1 & 5 \\
\hline 2 & $\mathrm{C} 1=5, \mathrm{C} 2=5, \mathrm{C} 3=21, \mathrm{C} 4=5, \mathrm{C} 5=2, \mathrm{CLASS}=3$ & 20 & 2 & 21 \\
\hline 2 & $\mathrm{C} 1=3, \mathrm{C} 2=3, \mathrm{C} 3=2, \mathrm{C} 4=3, \mathrm{C} 5=17, \mathrm{CLASS}=3$ & 16 & 2 & 17 \\
\hline 3 & $\mathrm{C} 1=2, \mathrm{C} 2=2, \mathrm{C} 3=6, \mathrm{C} 4=2, \mathrm{C} 5=15, \mathrm{CLASS}=3$ & 14 & 2 & 15 \\
\hline 3 & $\mathrm{C} 1=5, \mathrm{C} 2=5, \mathrm{C} 3=17, \mathrm{C} 4=5, \mathrm{C} 5=28, \mathrm{CLASS}=3$ & 24 & 5 & 28 \\
\hline 3 & $\mathrm{Cl}=9, \mathrm{C} 2=9, \mathrm{C} 3=29, \mathrm{C} 4=9, \mathrm{C} 5=1, \mathrm{CLASS}=3$ & 29 & 1 & 29 \\
\hline
\end{tabular}

Table 5.1: Example instances referenced to create Gen-Functions

When comparing the data in the table against the rules, it is obvious that referring to the rules alone would not provide sufficient information to calculate the values for the three functions. Unlike the previous example of rule-based generalization, this is more a process of data-based generalization. 
GF \$A: $[$ RGC(class $=$ General_Antecedent $)]$

GF \$B: [MNC(class $=$ General_Antecedent $)]$

GF \$C: $[\mathrm{MXC}($ class $=$ General_Antecedent $)]$

Unlike the NEV function, RGC, MNC, and MXC do not have parameters beyond which scope to use. Terms that contain these Gen-Functions compare directly against the result of the operation, and therefore can be used in any rule. This means, however, that there is no counter to indicate how useful or widespread a potential Gen-Function could become. Without this counter it is not possible to deterministically choose which one to insert into the population, so another step is performed in order to add weight to these candidates.

The extra step involves re-visiting the values stored in the temporary Gen-Function table. These values are the results of the evaluations against the rules in the candidate rule set, and identical results between rules will increase the score for the appropriate GenFunction. The most popular values in the previous table are:

- RGC; "5" has three instances

- MNC; "2" has four instances

- MXC; no duplicate values

Using this statistical knowledge we can anticipate what EvRFind will choose as values for the Gen-Functions. ( $\mathbf{\$ A}=\mathbf{5})$ and $(\mathbf{S B}=\mathbf{2})$ might be useful terms, but nothing is 
selected for Gen-Function \$C because there were no similarities discovered in the set of maximum values.

\subsubsection{Inserting Gen-Functions into the population}

If the Gen-Function candidate selection is successful then there can be any number of potential functions available. All of the scores are compared, and the highest score will determine which functions are inserted into the population. Typically there will only be one new Gen-Function created in a single generation, although there can be more than one if multiple functions are tied with the top score. There is no limit on the number of functions in this uncommon case.

After the top candidate functions are chosen they have to be inserted into the population. Unlike the in-place replacement process used when creating Meta-Variables, GenFunctions cannot simply be replaced syntactically within a rule. The reason for this is that no record exists for which terms contributed to the correctness or popularity of the function. For example, consider the following rules:

If $(A=B) \wedge(B=C) \wedge(X=Z)$ then $($ Class $=2)$

If $(\mathrm{A}=\mathrm{B}) \wedge(\mathrm{B}=\mathrm{C}) \wedge(\mathrm{X}>\mathrm{Z})$ then $($ Class $=3)$

During the Gen-Function selection process, \$A was created and represents three equal values. By inspection it is easy to see that the terms $(\mathbf{A}=\mathbf{B})$ and $(\mathbf{B}=\mathbf{C})$ are the same as $(\mathbf{A}=\mathbf{B}=\mathbf{C})$, and this is what was referenced when creating the function. The problem is, 
however, that there is no record of which terms should be replaced. The matter is further complicated by examples where the count for $\$ \mathbf{A}$ is higher than one, and record keeping for several sets of terms within the same rule would be computationally expensive. The solution is to generate a new set of rules containing each new function, and then evaluate them in the next generation.

As mentioned previously, EvRFind keeps track of which rules were used to create each Gen-Function, and this information is also available for the generalized version of functions. For each Gen-Function, one rule is created for each subset of terms in each of the source rules. Because the consequents of rules used to create a Gen-Function can differ, it is important to take this into consideration when creating replacement rules. For example, in cases where the empty set of terms is combined with the new Gen-Function, one new rule will appear for each different consequent found in the source rules.

The set of rules that would be created, based on the previous example, are:

If $(\$ A=1)$ then $($ CLASS $=2)$

If $(A=B) \wedge(B=C) \wedge(X=Z) \wedge(\$ A=1)$ then $($ CLASS $=2)$

If $(B=C) \wedge(X=Z) \wedge(\$ A=1)$ then $($ CLASS $=2)$

If $(X=Z) \wedge(\$ A=1)$ then $($ CLASS $=2)$

If $(B=C) \wedge(\$ A=1)$ then $($ CLASS $=2)$

If $(\mathrm{A}=\mathrm{B}) \wedge(\mathrm{X}=\mathrm{Z}) \wedge(\$ \mathrm{~A}=1)$ then $(\mathrm{CLASS}=2)$

If $(A=B) \wedge(\$ A=1)$ then $($ CLASS $=2)$

If $(A=B) \wedge(B=C) \wedge(\$ A=1)$ then $($ CLASS $=2)$ 
If $(\$ A=1)$ then $($ CLASS $=3)$

If $(A=B) \wedge(B=C) \wedge(X>Z) \wedge(\$ A=1)$ then $(C L A S S=3)$

If $(B=C) \wedge(X>Z) \wedge(\$ A=1)$ then $($ CLASS $=3)$

If $(X>Z) \wedge(\$ A=1)$ then $($ CLASS $=3)$

If $(B=C) \wedge(\$ A=1)$ then $($ CLASS $=3)$

If $(\mathrm{A}=\mathrm{B}) \wedge(\mathrm{X}>\mathrm{Z}) \wedge(\$ \mathrm{~A}=1)$ then $(\mathrm{CLASS}=3)$

If $(\mathrm{A}=\mathrm{B}) \wedge(\$ \mathrm{~A}=1)$ then $(\mathrm{CLASS}=3)$

If $(A=B) \wedge(B=C) \wedge(\$ A=1)$ then $($ CLASS $=3)$

The rules vary in specificity, and with having the reader's knowledge it is trivial to see that several of these rules make very poor use of the new Gen-Function. However, the rule evaluation process in the next generation will score each accordingly. One that will obviously do poorly is:

If $(\$ A=1)$ then $($ CLASS $=2)$

This rule will score badly because ( $\mathbf{S A}=\mathbf{1})$ is also true for at least some instances where $($ class $=\mathbf{3})$ is true. A good guess at the two best rules is:

If $(X=Z) \wedge(\$ A=1)$ then $($ CLASS $=2)$

If $(X>Z) \wedge(\$ A=1)$ then $($ CLASS $=3)$ 
Although these might not describe the actual concepts, they represent a generalization of the two example rules that still expresses the relationship between $\mathbf{X}$ and $\mathbf{Z}$.

In any event, all of the rules will be scored and the evolutionary search will continue. Rules that are in conflict with each other, or are inferior for other reasons, will be excluded from the hierarchy and eventually removed from the population. Other rules will be promoted, modified, and continue to evolve unless replaced by better ones.

Several good examples of the creation and use of Gen-Functions can be seen in the experiments on the Poker Hand Dataset, specifically in Chapter 6.7, Using EvRFind for Rule Discovery, starting on page 193.

\subsubsection{Post discovery usage}

In addition to the original rules being replaced there may be a number of undiscovered rules that are also incorporated automatically. Although there is a risk of overgeneralizing, this result will be penalized during the evaluation stage. Similarly, if other rules in the population become redundant because of a new rule then the evolutionary component in EvRFind will have them replaced during the next generation.

\section{$\underline{\text { Valid operators }}$}

As is the case with regular variables, each Gen-Function has a valid set of operators that can be used within a term. The list of operators is set by the creation process, and is determined by taking the intersection of all operators allowed by the attribute class. For 
example, if the "general_antecedent" attribute class has several variables then each of their operators will be checked. Only operators compatible with every variable will be available for use by the Gen-Function.

Some Gen-Functions have a default operator. NEV, as an example, has ' $=$ ' as the default operator. This operator will be used to create the initial set of rules. If the default is not specified then one of the operators is chosen at random. In the later stages of evolution when mutation operations are being performed, the default value for a Gen-Function is not considered to be more important than the others.

\section{Mutation}

With the possibility that a term containing a Gen-Function can be subject to mutation, it means that the upper and lower bounds on the Gen-Function return value have to be set. The method for determining bounds is specific to each Gen-Function, but as an example, the NEV case is trivial.

There is nothing to consider when setting the lower bound for the NEV function because it is always zero. It is possible to state that "There are no pairs of equal values in this set", while it does not make sense to state that "There are fewer than zero equal values".

The upper bound is half of the number of attributes that exist in the attribute class. If the parameter for the NEV function is higher than the fifty percent mark then it is higher than it needs to be. For example, $\{[\mathrm{NEV}($ Class $=$ General_Antecedent $)=3]=1\}$ means that 
there is one set of triplicate values in the set. If there are five variables in the attribute class then the only possible values for this are 0 and 1 . Using half of the class makes the bounds 0 and 3 . It is safer to have a higher upper bound because erroneous results will receive a low fitness and will subsequently be ignored, but at the same time it should not be set unnecessarily high because it can make the process less efficient.

\section{Optimizing efficiency}

Gen-Functions will only be evaluated once for each data instance during the course of the search. The result is stored internally to the Gen-Function and recalled if requested again. The value is initially marked as unknown, and is only evaluated for the first time upon demand.

\subsubsection{List of Implemented Gen-Functions}

The following table of Gen-Function primitives have been implemented and tested in the current version of EvRFind. A list of functions that are planned for future implementation can be found in Appendix G: Future Gen-Function Primitives, starting on page 313.

\begin{tabular}{|c|l|l|}
\hline Symbol & Parameters & Description \\
\hline AVA & Attribute list & Average of values in the list of attributes \\
\hline AVC & Attribute class & Average of values in the specified class \\
\hline LFA & Attribute list & Return the least frequent value in the attributes \\
\hline LFC & Attribute class & Return the least frequent value in the attribute class \\
\hline MFA & Attribute list & Return the most frequent value in the attributes \\
\hline MFC & Attribute class & Return the most frequent value in the attribute class \\
\hline MNA & Attribute list & Minimum value in the specified attributes \\
\hline MNC & Attribute class & Minimum value in the specified class \\
\hline MXA & Attribute list & Maximum value in the specified attributes \\
\hline MXC & Attribute class & Maximum value in the specified class \\
\hline
\end{tabular}




\begin{tabular}{|l|l|l|}
\hline NEA & Attribute list & Return the number of equal values in the attributes \\
\hline NEV & Attribute class & Return the number of equal values in the class \\
\hline RGA & Attribute list & Range of values in the list of attributes \\
\hline RGC & Attribute class & Range of values in the specified class \\
\hline SUM & $\begin{array}{l}\text { Attribute list, } \\
\text { constant }\end{array}$ & $\begin{array}{l}\text { Summation of the values in attributes and optionally } \\
\text { a constant }\end{array}$ \\
\hline
\end{tabular}

Table 5.2: Implemented Gen-Function Primitives

\subsubsection{Hill-Climbing}

The tasks being performed during intergenerational processing have included optimization, learning from experience through the creation of building blocks, and generalization. These tasks all share the goal of improving the search, but the primary mechanism is still an evolutionary search. As discussed in [Zhang, Ishikawa, 2004; Prügel-Bennett, 2004] the genetic algorithm is proficient at coming close to a solution, but lacks the fine tuning operators required to make small steps towards the optimal solution.

The hybrid (HGA) solutions used in published works such as [Gwee, Chang, 2003; Kotecha, Gambhava, 2003] involve the use of a genetic algorithm followed by a local search technique. The local search component is typically a standard Hill Climbing algorithm, and is used to finely tune the potential solutions. In an attempt to benefit from this method, EvRFind can optionally perform a hill climbing search at the rule level between generations. 


\subsubsection{Description}

A search using Hill Climbing relies on heuristics to assign a score to potential solutions. In the case of a rule learner, each rule receives a score, and changes to the rule typically result in a change of score. A rule with a high score might be close to an optimal solution, while a low-scoring rule might be largely incorrect or invalid. In EvRFind, the score is the fitness of the rule.

The goal of a Hill Climbing algorithm is to improve candidate solutions by making minor modifications, and then accepting these changes if the modified solution is closer to the goal state. If none of the changes result in a higher score then the Hill Climbing for that solution is halted.

The first step in the Hill Climbing process is choosing a candidate solution. As with other tasks performed during the intergenerational processing stage, Hill Climbing is computationally expensive and will only be used to expand the search in a limited area during each generation. 


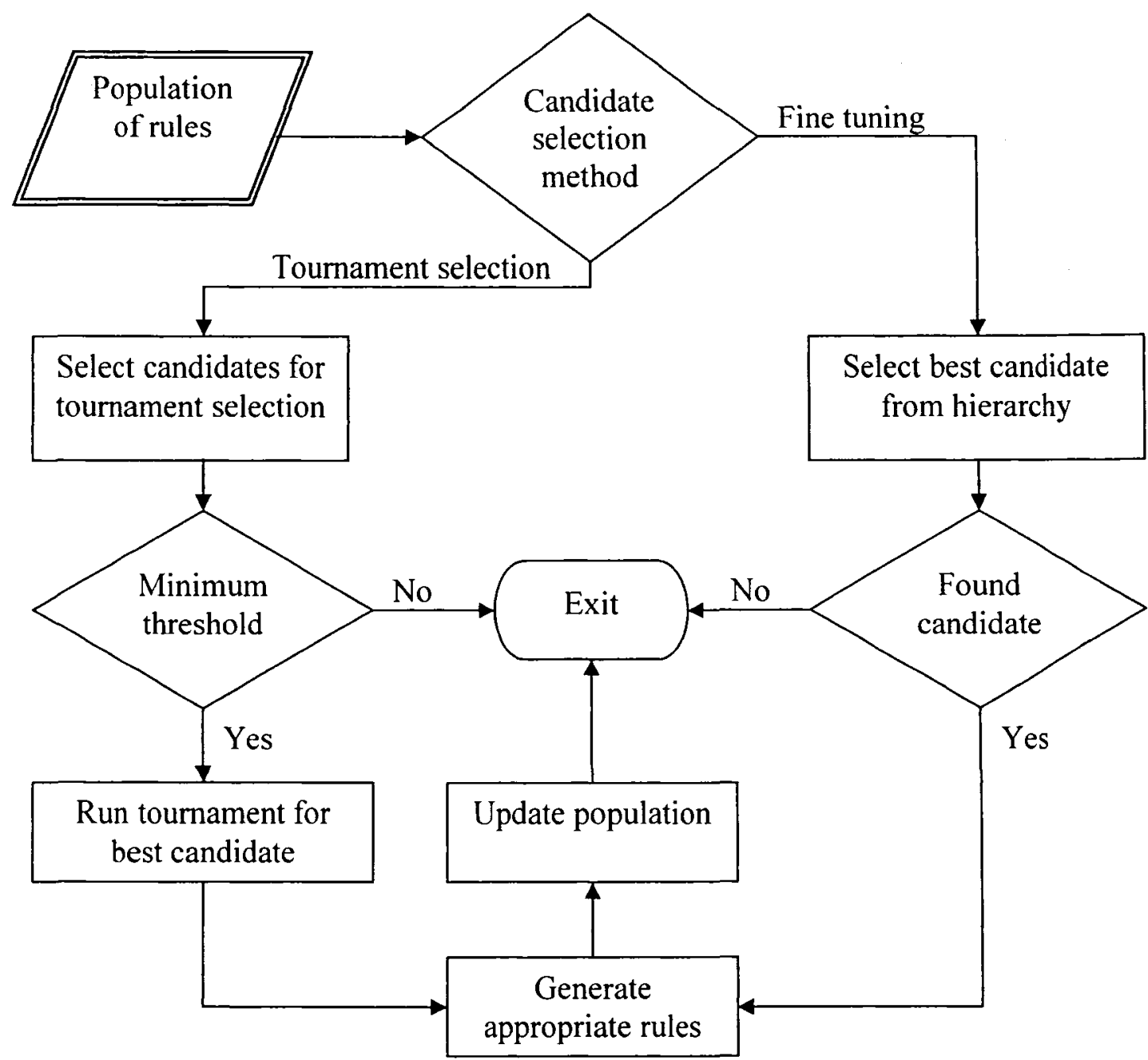

Figure 5.9: Hill climbing overview

This chart is the continuation of a previous chart, the Intergenerational processing overview, on page 112 . 


\subsubsection{Candidate selection}

Two methods for determining candidates are available in EvRFind, and the option is specified in the configuration for each experiment. The first is a deterministic method that tries to select the rule with the highest fitness.

\section{Candidate selection by highest fitness}

Starting at the top of the hierarchy, the selection process traverses the rules until one with $90 \%$ or higher confidence is found. Provided that this rule has not been the subject of Hill Climbing in a previous generation, it is selected as the only candidate. If chosen in a previous generation then the selection process continues. If the search for a candidate continues until a rule with less than $50 \%$ confidence is found, then the selection process terminates and EvRFind continues without Hill Climbing during this generation.

During experiments for comparative evaluation, the first selection method successfully fine-tuned several rules during each experiment. In some cases the higher-fitness rules were found in earlier generations, and the final hierarchies were improved in general. With the fine-tuning benefits of Hill Climbing being realized, a second selection method was created to aid with the explorative part of the search.

\section{Candidate selection using a tournament}

Instead of always searching for the rule with the highest fitness, the second selection method is more probabilistic. The process is based on tournament selection, which is a competition between a small set of randomly chosen rules. When the fitness scores of the 
rules in the competition are compared, the rule with the highest fitness is considered to be the winner. A smaller competition size means that the likelihood of a less-fit rule being chosen is greater. Conversely, in larger competitions, there are more randomly chosen rules. The more rules there are in the tournament, the greater the chance of it being a high-fitness rule that wins the competition. Trivially, if the tournament size is the equal to the entire population then the best individual will be chosen.

Selection of rules for the tournament is done by randomly choosing rules from the hierarchy. The process will examine up to twenty rules at most. If a rule has not been selected for Hill Climbing in a previous generation then it will be added to the competition. Once there are five rules in the competition the selection process halts. If the set of twenty first-chosen rules that are examined does not contain at least five that are suitable for the tournament, then the tournament size will be lower for this generation. It is possible, depending on the size of the population and the number of generations passed, that Hill Climbing might not take place because there are no rules in the tournament.

If after selection there is more than one rule in the competition then the tournament takes place. The fitness scores for each rule are compared and the most-fit rule is selected. Unlike standard tournament selection, it is still possible for the least-fit rule in the population to be selected. This can happen in the case where nineteen of the random selections had been chosen in previous generations, and the only un-climbed selection is the least-fit rule. 


\subsubsection{Hill Climbing as a Rule Mutation Operator}

Using Hill Climbing as a technique for fine tuning or exploration in a rule discovery system can be done through minor modifications to existing rules. Changes can be made by adding or removing terms, or with greater resolution, modifications within terms are also possible. In EvRFind, several operations are performed on candidate rules that generate many sibling rules, each one typically containing a single change. These operations include the following independent steps:

1. Make a copy of the original rule.

2. For a single rule with $N$ terms, create $N$ rules that are identical to the original rule, but each missing a different term. This step is skipped for rules that contain exactly one term.

3. For each variable in each term, create siblings that use every other compatible variable. This does not include Meta-Variables or Gen-Functions.

4. For each constant value in each term, create siblings that use every compatible variable in place of the constant. This does not include Meta-Variables or GenFunctions.

5. Create sibling rules that are identical except that each one is appended with a Meta-Variable. For one rule, for each Meta-Variable, another variant rule is created.

6. For each comparison operator in each term, create sibling rules using every other compatible operator. (Ex: swap '=' for '>', '<', etc, as long as they are compatible with the original term). 
7. For each integer or real value, add sibling rules with various mutations on the values:

a. If there are less than twelve valid integer values then add a new rule for each one of them.

b. If there are less than five integer values between the lower bound and the current value, then add one instance for each value in between.

c. If there are less than five integer values between the upper bound and the current value, then add one instance for each value in between.

d. For real values, or integer values with sufficient difference between the bounds and the current value, take five values on each side of the current value. Each new value is calculated by adding or subtracting $15 \%$ of the difference (between current value and the appropriate bound) in increments.

\section{First example}

Lower bound $=1$, Upper bound $=13$, Current value $=10$

Values: $8,7,5,4,3,11,12$, and 13 .

\section{Second example}

Lower bound $=0$, Upper bound $=1500$, Current value $=1097$

Values: 932, 767, 603, 438, 274, 1157, 1217, 1278, 1338, and 1399. 
8. Hill Climbing a rule that contains a Gen-Function is only done where the result is compared to an integer or real value. Several new rules will be created by modifying the constant value using the following steps:

a. Each new rule will contain a different constant within the term that contains the Gen-Function.

b. The goal is to add, at most, ten new rules. If the Gen-Function has result bounds that differ by more than ten then the number of new rules is limited by spacing the values apart using the increment: [ (upper_bound-lower_bound) / 10 ].

c. The comparative operator will be the default for the Gen-Function. If the Gen-Function does not have a default operator, then one is chosen at random from the list of those that are allowed.

9. Create sibling rules that are identical except that each one is appended with a Gen-Function. For each rule, follow the steps as specified by step \#8. The difference is that a new term containing a Gen-Function is being added as opposed to an existing one being modified.

The result of executing these steps is sometimes a large number of rules, all of which are just slightly different from the original candidate. Depending on factors such as the number of variables, their valid ranges, and the number of Gen-Functions, the set of mutated rules can number in the dozens or even hundreds. It is for this reason that only one rule will be Hill Climbed during each generation. 
This addition of many new, but slightly different, rules has the same effect as performing many different mutations at one time. All of them, including the original rule, will be evaluated and competing for fitness in the next generation. If one of the mutations has a positive impact then it may replace the original. In some cases it is possible that a mutation will have a positive effect, but due to the nature of the change it will significantly impact a different target concept. In this rare situation the original rule will address instances from the original target concept, while the new rule will address instances from a different target concept. As a result, the original will not be displaced and both persist in the hierarchy.

If the original candidate rule is maintained in the next population then it will be marked as Hill Climbed, and it will not be selected for the same purpose again. Similarly, if one of the mutated rules exists in the next population then it will be marked as a product of Hill Climbing, and as such might be Hill Climbed during some future generation. In the current version of EvRFind, the recognition that a rule is a product of Hill Climbing is for informational purposes only and has no effect on the remainder of the search.

\subsubsection{Example}

To illustrate the full effects of performing this operation on a specific rule, the following example includes three terms of differing types. Also, a variety of higher level functions are available for use by the process. 


$$
\begin{aligned}
& \text { GF: \$A }[\text { RGC(class = Rank })] \\
& \text { GF: } \$ B \text { BNEV(class = Rank })=2\{\text { Variable }\}] \\
& \text { MV: \#C }[(\mathrm{C} 1>\mathrm{C} 3) \wedge(\mathrm{C} 5=\mathrm{C} 4)] \\
& \mathrm{MV}: \# \mathrm{D}[(\mathrm{C} 1>\mathrm{C} 5) \wedge(\mathrm{S} 2 !=\mathrm{S} 4)]
\end{aligned}
$$

The rule to be climbed is:

If $(\mathrm{C} 1>\mathrm{C} 3) \wedge(\mathrm{C} 2<5) \wedge(\$ \mathrm{~B}=1)$ then $(\mathrm{CLASS}=1)$

In this example the Hill Climbing procedure creates a total of 49 rules, including a copy of the original. Each of the 48 rules that are not equal to the original represents a small mutation from it. The effect is the same as expanding the search through a large number of syntactically similar, but non-identical rules.

A complete list of the variables, integer ranges, and the rules generated by EvRFind for this example is available in Appendix A: Hill Climbing example, starting on page 295.

\subsection{Automatic Consequent Balancing}

\subsubsection{Description}

Automatic consequent balancing refers to a process within EvRFind that is used to focus the search in classification problems. The general idea is to monitor what classes are being correctly classified by the rules in the population, and then focus the computational effort by directing the search to concepts that are not being adequately covered. 
This process is somewhat similar to the concept of boosting, where a meta-search technique makes use of many weaker rule-learning methods and combines their results through a series of weighting measures. Algorithms that employ boosting, such as AdaBoost [Freund, Schapire, 1997], perform better in many domains when compared to conventional search algorithms.

\subsubsection{Implementation details}

During classification problems, EvRFind monitors the coverage achieved by each of the rules in the population. This process does not focus on an individual rule, but rather the cumulative effect of all rules that answer the same class because of an in-common consequent. The coverage is tallied and compared against all of the instances in the dataset that belong to the same class. The result is an indication of what coverage is currently being attained for each of the target concepts within the domain.

This information concerning the performance of the current population is particularly useful in cases where the target coverage is non-uniform between classes. Specifically, in EvRFind, when it is found that a concept is being $100 \%$ covered by the existing set of rules then there is an intentional effort to re-direct the search. This is done by modifying any new rule that attempts to answer a target concept that is already fully covered.

The goal of re-directing the search is to narrow the focus by excluding classes that the system already answers adequately. Essentially, it encourages the creation of rules that address poorly-classified concepts. The drawback to re-directing the search entirely is 
that classes with $100 \%$ coverage may be answered by a set of rules that are sub-optimal and would otherwise still benefit from refinement. To deal with this problem, EvRFind probabilistically chooses whether to refine or explore.

In the cases where EvRFind chooses to refine a concept, the new rule is allowed to stay and compete with others that have the same consequent. Alternatively, if EvRFind chooses further exploration then the rule is modified such that it will address one of the targets with lesser coverage.

The reason that this choice is made probabilistically, rather than using an approach that allocates effort proportionally between exploration and refinement based on what is required at that time, is to save the overhead required to make this more informed decision. This overhead includes managing the number of times that this occurs within the generation, and ensuring that computing cycles are assigned accordingly for each target concept. Empirically, the random choice appeared to work sufficiently well and the more complex method was not implemented.

\subsubsection{Experimental evaluation}

Several experiments with a variety of datasets were performed in order to configure, test, and evaluate the automatic consequent balancing technique. The variable that changed between experiments was the setting that specifies the probability of choosing exploration versus refinement. 
As expected, the results of these experiments indicated that negating refinement had a detrimental effect on the end results. In contrast, if refinement was too heavily preferred over exploration then the progress was slower on average. As a result, the default value for this parameter is $50 \%$.

One of the observations from these experiments was that all of the results were at least as good as those discovered when automatic consequent balancing was not being used. In most cases the resulting models were either better in terms of performance, or they evolved in a shorter period of time.

\subsection{Lifetime duplicate check}

\subsubsection{Description}

Implemented in EvRFind is a mechanism to remove duplicate rules from within the population. Designed and used as a diversity-maintaining process, the original experiments showed that it contributed positively to the rule discovery process. Similar work in [Zhang, Ishikawa, 2004] has also shown that removing redundant members from within the same population helps to make the search more efficient.

Unfound in published work on evolutionary algorithms is the creation and maintenance of an ancestral history. In evolutionary systems that employ techniques such as genetic algorithms, genetic programming, and hill climbing, there is no history of candidate solutions maintained. This is in contrast to other search methods, such as Depth-first- 
search, Breadth-first-search, and the $A^{*}$ algorithm, where the process can be made such that a syntactically identical solution path will never be re-discovered. Even with these algorithms it can still occur if the number of states in the closed list is limited. An example of this is the algorithm proposed in [Schroedl, 2005]. In this paper the problem is described as "leaking back", and the authors state that it should be avoided due to the higher cost required to explore previously seen nodes.

In addition to removing duplicate members from within the population during a single generation, this feature maintains a history used for cross-referencing against every solution that has been discovered during the course of the experiment. In EvRFind this equates to maintaining a list of every rule that has been evaluated during the course of the current experiment.

Maintaining and referencing such a history is expensive in terms of both memory and computational effort. Initial experiments had shown that the concept was feasible, and had a positive effect on the results. Once this was realized, an effort was made to make the component more efficient.

\subsubsection{Implementation details}

The most efficient model evaluated was a trie data structure that could be expanded and referenced, but not modified. The trie structure is most useful when the keys are of varying lengths and some keys are non-existent. In the case of EvRFind, a hash value for 
each rule is created and used as the key within the trie. Looking up a rule takes, at most, $\mathrm{O}(\mathrm{m})$ time, where $\mathrm{m}$ is the length of the hash value for the rule.

At the end of each generation the entire trie is written to a file that can later be used for audit or restart purposes. This file is incremental, and a collection of files can be used to show how a search has progressed through the generations.

The hash value for each rule is a simplified version of the rule, with several of the formatting characters removed. In addition to removing non-relevant characters, the terms and their contents are sorted so that hash values for semantically equivalent rules will be the same. There is still enough information to re-create the rule, although it is not always possible to return to the identical syntactic version.

In the current system, not being able to re-create a rule in its original form does not appear to cause any problem. Theoretically, however, it could affect the evolutionary process where a new population is seeded with rules from a previous experiment. If the rules being inserted into the new experiment are re-created using the trie alone then some of the terms, as well as their contents, will be sorted differently. Although semantically identical, probabilistic genetic operations such as crossover and mutation may behave differently on these variations. For this reason the EvRFind restart functionality does not rely on the trie to reconstruct the population before continuing an experiment. 
Associated with rules in the trie structure is a state that indicates what the current status of the rule is. The possible states are:

Exist_no; The rule has never been part of the hierarchy.

Exist_active; The rule is currently part of the hierarchy.

Exist_discarded; The rule was selected for removal from the hierarchy. This happens at the end of every generation when the population is trimmed. Only the lowest-fitness rules are discarded.

Exist_died; The rule was part of the hierarchy, but was not selected for a successive generation. This differs from the Exist_discarded state because it was simply not selected, rather than intentionally discarded. This occurs due to the probabilistic nature of fitness proportional selection.

Exist_revived; The rule was re-created and allowed back into the hierarchy. This can only happen when the rule is in the Exist_died state. As mentioned previously, rules marked as revived might be syntactically different from the original.

Keeping track of these states is necessary for the algorithm to work properly, but it also allows for additional statistics to be compiled. This information can be used for tracking progress, and may lead to improvements in future versions of EvRFind. 
A module within the user interface allows for browsing a single trie file, which is a snapshot for a single generation. Available for display is the entire history up to that point, including the states of all rules encountered during the course of the experiment. Planned for a future release is a tool that will compare two or more generations, and show the changes that occurred between the start and end periods.

\subsubsection{Organization of Experiment Files}

With the introduction of the lifetime duplication check and the trie data structure, the number of files stored for each experiment is significant. To better manage the files associated with each experiment, EvRFind creates a folder for each one. All of the files for a single run are in the folder, including multiple experiments with the same options but executed with different random seeds.

\subsection{Post-processing stage}

Some functionality, primarily in reporting, occurs during the post-processing stage of EvRFind. This phase starts once the evolutionary search concludes and the hierarchy is in the semi-final form. These optional modules can be used at any time after an experiment completes as long as the results are still available.

\subsubsection{Viewing Confusion Matrices}

In addition to general reports and statistics concerning the results, EvRFind creates a confusion matrix to show how accurately each individual class is being classified. The 
matrix is in the Weka style, where the right column indicates the class of the example instance, and the top row indicates what the corresponding example instance was classified as by EvRFind.

\section{Example}

\begin{tabular}{|c|c|c|c|c|c|c|c|c|c|c|}
\hline$<0\rangle$ & $<1>$ & $<2>$ & $\langle 3\rangle$ & $<4>$ & $<5\rangle$ & $\langle 6\rangle$ & $\langle 7\rangle$ & $<8>$ & $\langle 9\rangle$ & \\
\hline 12493 & 0 & 0 & 0 & 0 & 0 & 0 & 0 & 0 & 0 & $<-<0\rangle$ \\
\hline 0 & 10599 & 0 & 0 & 0 & 0 & 0 & 0 & 0 & 0 & $<--\langle 1\rangle$ \\
\hline 0 & 0 & 1206 & 0 & 0 & 0 & 0 & 0 & 0 & 0 & $<--\langle 2\rangle$ \\
\hline 0 & 0 & 0 & 513 & 0 & 0 & 0 & 0 & 0 & 0 & $<--\langle 3\rangle$ \\
\hline 12 & 0 & 0 & 0 & 93 & 0 & 0 & 0 & 0 & 0 & $\langle--\langle 4\rangle$ \\
\hline 54 & 0 & 0 & 0 & 0 & 54 & 0 & 0 & 0 & 1 & $\langle--\langle 5\rangle$ \\
\hline 0 & 0 & 0 & 0 & 0 & 0 & 36 & 0 & 0 & 0 & $<--\langle 6\rangle$ \\
\hline 0 & 0 & 0 & 0 & 0 & 0 & 0 & 6 & 0 & 0 & $<--\langle 7\rangle$ \\
\hline 0 & 2 & 5 & 0 & 110 & 3 & 0 & 0 & 0 & 5 & $<--<$ \\
\hline 5 & 0 & 0 & 0 & 0 & 0 & 0 & 0 & 0 & 5 & $<--<$ \\
\hline
\end{tabular}

Rule count: 100

Record count: 25010

Rules used: 20

-> Rule \#1: 12493 correct, 71 incorrect

-> Rule \#2: 10599 correct, 0 incorrect

-> Rule \#24: 0 correct, 5 incorrect

The data that follows the confusion matrix is the number of rules in the hierarchy, the number of records in the dataset, and the number of rules that were actually used to 
positively classify instances. Other information, such as the number of rules that do not match the antecedents of any examples or the number of rules that are completely redundant, is not displayed.

Following the summary information are specific details on each of the rules that were used to create the confusion matrix. This includes rules that are $100 \%$ correct, partly correct and $100 \%$ wrong. The only requirement is that they contributed to the figures within the matrix.

Looking at the confusion matrix can offer a high-level insight as to how well the model predicts the data. In the above matrix, for example, two classes that stand out are $<6>$ and $<8>$. For class $<6>$, there are 36 instances correctly classified, and no mistakes are made with examples that belong to class $<6>$. Furthermore, no example belonging to another class is incorrectly classified as $\langle 6\rangle$. In the case of class $\langle 8\rangle$, all of the examples are misclassified, and there were no rules that even attempted to place a single example in class $<8>$.

The ideal confusion matrix would have values that total $100 \%$ of the dataset, and only have non-zero values in the diagonal line running from the upper left to the lower right of the matrix. 


\subsubsection{The Predict feature}

During the development of EvRFind it was primarily the case that sets of rules were being evaluated against a testing set, where the correct classes were included and the predictive model could be immediately evaluated. However, there were some datasets that consisted of example instances for which no class was specified.

This category of instances required the development of the Predict feature. Essentially, these instances still need to be classified, but rather than instantly comparing the results to determine the overall predictive accuracy of the model, the class predictions simply have to be reported.

\subsection{Execution time}

The execution time for EvRFind refers to the computing time required to create a hierarchy for a specific training set. Being a search technique that uses probabilistic methods to evaluate and evolve generations of solutions, the process naturally requires more computational effort as compared to algorithms, such as $\mathrm{C} 4.5$, that rely on straightforward deterministic measures such as information gain. In many cases, depending on additional factors that are described below, EvRFind is much slower than other probabilistic algorithms as well.

The amount of time for any given search varies depending on factors including the nature of attributes used to describe the domain, the size of the training set, the number of rules in the population, and the options that are used during the search. Options that can be 
used in the intergenerational processing stage, such as Hill Climbing and automatic concept generalization, require a greater amount of computing effort. Consequently, any search that employs these options will take longer to complete, and it can often be measured in terms of an order of magnitude slower. As an example, some simple datasets can be fully searched within a minute, while larger and more complicated datasets can take several hours to fully analyze.

Although the running time is longer than that of some other methods, the evolutionary nature of the process precludes certain steps in the data staging process. Data staging refers to any pre-modification of the data in order to make it more suitable for searching. Examples of this are the manual removal of obviously non-relevant or duplicate attributes, and the creation of aggregate variables that are derived from two or more existing attributes.

Once a predictive model has been created with EvRFind, the execution time required to evaluate the model against unseen data is similar to that of other algorithms. Effectively this means that after a model is built, there is no time penalty incurred by using EvRFind. 


\section{Chapter 6: The Poker Hand Dataset}

\subsection{Motivation for creating the dataset}

To properly evaluate the performance of a data mining algorithm it is necessary to have an understanding of the data being examined. Without a reasonable level of knowledge it can be very difficult or impossible to recognize how accurate or useful a discovered concept is. Without independent verification the concept might not be recognized as incorrect or even that it is valid but represents common knowledge and is of no interest in the respective domain. For rule based systems such as EvRFind, verifying the results is a non-trivial task in itself.

Particularly for non-experts in the domain being examined it is difficult to judge the value of any given rule, and may require the opinions of several knowledgeable individuals. These concerns are echoed by the authors in [Dryl et al, 2004], where they also note that every claim made by their system must eventually be verified by a human expert. Depending on the problem domain these experts might be difficult to find and seek opinions from. Of more concern is that, at least in some cases, a suitable expert might not exist at all. Even in domains where experts are available there are cases where classification is subjective and opinions vary between people. Considering the additional effort and associated problems with consulting experts in the appropriate areas, it makes it difficult to evaluate data mining algorithms on real datasets. 
One way to address the problem of evaluating results from a rule induction algorithm is to generate a set of random data that conforms to a pre-specified set of known rules. The data can optionally contain noise, incorrect and missing values, as well as non-essential attributes. The quality of the algorithm can be judged by the correlation between the original rules and the discovered rules. The drawback to this method stems from the fact that these datasets are purely abstract and have no real-world meaning.

A popular example of using synthetic datasets to evaluate data mining and machine learning algorithms is the Monk's problems [Thrun et al., 1991]. There are three Monk's problems, each one containing both training and testing sets. The data is generated using one rule for each set, and the third problem intentionally contains class noise. EvRFind is evaluated using the Monk's problems in Chapter 7.3, The Monk's Problems, starting on page 236 .

The ultimate goal when using a synthetic dataset is to discover the original rules or concepts. Depending on the grammar available to the algorithm it may not be possible to exactly re-create the defining set of rules, but variants of the originals are often acceptably close. In some cases it might take several rules to describe a concept that was originally described using only one, and in other cases a variant could be an optimized version of the original.

Similar to the case of real-world datasets it is sometimes difficult to evaluate the correctness of discovered rules. Without an exact match of the original there has to be 
some analysis, and this can be difficult considering there is no real-world meaning and the reviewer may not have any opinion on validity or accuracy.

Another problem with synthetic datasets is that they can represent a search space that is unbounded and unfair to some learning algorithms. If integer bounds (ranges) or the precision for real values are large then it makes it very difficult for algorithms that are not capable of relational learning. This problem was described in [Cattral, Oppacher, Deugo, 2001], and was highlighted using the polygon dataset. This synthetic dataset was designed specifically to show that learning relations between attributes is necessary to achieve reasonable accuracy in some domains. In this thesis there are more experiments with the polygon dataset in Chapter 7.6, Polygon Dataset, starting on page 260.

Ideally a synthetic dataset will contain data that reflects several concepts of varying difficulty. These concepts correspond to classes and there are many instances used as examples for each class. Difficulty, in this context, refers to how much computational effort is required for a learning algorithm to discover the underlying concepts for one particular class. If the learning algorithm is not capable of creating a predictive model because it lacks something in the grammar, then it is not just difficult but infeasible.

Although difficulty will vary between algorithms and techniques, there are several dataspecific factors that contribute to how difficult learning is. A significant factor is unbalanced data, which means that different numbers of example instances represent the different classes. This problem is described in Chapter 3.4.4, Dataset Imbalance, starting 
on page 37 . The reason for the dissimilarity in numbers might be representative of the data as a whole, or instead because the sampling of data did not capture the true representation.

Because dealing with unbalanced data is a common problem that has a noticeable effect during training [Kubat, Matwin, 1997; Chawla et al., 2002; Drummond, Holte, 2003], synthetic datasets should have this property as well. Additionally, classes can be more difficult to discover if the data contains noise, missing values, or attributes that have no predictive value.

Although knowledge discovery needs to be difficult when using a synthetic dataset, the domain should be simple enough that analysis of the results is easy. The data must be suitable for both supervised and unsupervised learning, and finally it should be something that can map onto real-world problems. These are all goals that are achieved by using the Poker Hand dataset.

\subsection{Description}

\subsubsection{General Description}

The Poker Hand dataset, which is more completely described below, was generated with the intention that it be difficult to discover the underlying concepts yet easy to analyze them. Because of the way that the problem is represented it is difficult to discover rules that can correctly classify poker hands, although the simple nature of the game makes it 
trivial for the human analyst to validate potential rules objectively. The solution space is bounded because there are a finite number of valid poker hands. A valid hand is restricted to five unique cards in any position drawn from a standard deck of 52 playing cards.

Further examples showing the advantages of the poker hand dataset are described, including how it can be readily mapped onto other real-world problem domains such as resource allocation in a network, and how it could be used for rule evaluation even in unsupervised learning tasks.

Universally there are many ways to score poker hands. The most common scoring method is described by the following table:

\begin{tabular}{|l|l|l|l|}
\hline S & \multicolumn{1}{|c|}{ Name } & \multicolumn{1}{c|}{ Description } & \multicolumn{1}{c|}{ Example } \\
\hline 9 & Royal Flush & Ace $\rightarrow$ Ten of same suit & AH KH QH JH 10H \\
\hline 8 & Straight Flush & Five sequential ranks, same suit & 4C 5C 6C 7C 8C \\
\hline 7 & Four of a kind & Four of the same rank & 2H 2D 2S 2C 8S \\
\hline 6 & Full house & Three of a kind plus one pair & 3D 3C 7H 7S 7D \\
\hline 5 & Flush & Five cards of the same suit & 2C 3C 6C 9C AC \\
\hline 4 & Straight & Five sequential ranks & 3C 4C 5D 6H 7D \\
\hline 3 & Three of a kind & Three equal ranks & 5H 5S 5D 3C 7H \\
\hline 2 & Two pairs & Two pairs of equal ranks & 4H 4S 9D 9S 7C \\
\hline 1 & One pair & Two equal ranks & QH QD 2C KH 9S \\
\hline 0 & Nothing & No useful cards in the group & 2S 3D 6H 9C 10H \\
\hline
\end{tabular}

\section{Table 6.1: Simple Poker Hand scoring}

In the table above, the example hands are sorted and show all of the important cards in bold face. The actual ordering of the cards within the hand does not matter. For example, a three of a kind is obtained regardless of what positions the three like-ranked cards are in. A more complete description of the poker hand scoring system is available in Appendix C: Definition and Scoring of Poker Hands, starting on page 300. 
The $\mathbf{S}$ (score) column corresponds to a value that ranks the ten different possible poker hands. Because each set of five cards is a final hand, where no changes can be made, it is reasonable to use this simple scoring system. In cases where substitutions of cards can occur after scoring, it is important to recognize that incomplete hands can also have value. For example, if a hand consists of four of the five cards required for a simple straight, and the mismatched card has the possibility of being exchanged for the missing card, then it is important to recognize this potential.

An example of a scoring system that assigns credit to potentially winning hands is the Video Poker scoring system. This is more completely described in Appendix E: Video Poker Scoring System, starting on page 308. For the remainder of this description it is assumed that the simple poker scoring system is being used.

\subsubsection{Reasons that this is a difficult problem}

With respect to a dataset to be searched, the concept of difficulty is defined by what issues might present problems for the search algorithm. These problems are of varying degrees of difficulty and the ability to overcome them often depends on the technique being applied. The factors that make this dataset difficult are the size of the dataset, overlap between concepts, the imbalance in the domain, non-standard relationships between attributes for some classes, overloaded attribute values, and the way in which the data is represented. 


\section{$\underline{\text { Size of the dataset }}$}

Each hand consists of five cards that are not sorted by suit or rank, which means that the entire dataset contains approximately 311.8 million instances.

\section{Overlap between classes}

It is important to notice that these scores, which become classes in our application of this dataset, are mutually exclusive of each other. There are several cases where one class is a subset of another, but the two are still considered to be distinct. Two examples of this are Flush and Straight.

A flush hand is exactly five cards of the same suit, and a straight is five cards of mixed suits that are ranked sequentially and without gaps. A straight flush is a combination of the two: five cards of the same suit that are sequentially ranked. A royal flush is a type of straight flush, but restricted to ranks (Ten, Jack, Queen, King, and Ace). Although a royal flush is a straight flush, and it is also a flush, and also a straight, it is necessary that it be properly distinguished from these other classes.

Unlike the straight flush or royal flush, there are proper subsets that can also be equal to valid classes. This is true in the case of a full house, where the five cards are comprised of a single pair and a three of a kind. 


\section{Dataset imbalance}

As can be seen in the next section, 6.2 .3 , Statistics, starting on page 175, there is a large variation between the numbers of instances in the different classes. Generally speaking, the poker hand dataset contains a high degree of imbalance.

\section{Non-standard relationships}

At first glance it might seem that relational learning algorithms have a large advantage because of their ability to more easily discover concepts such as "pair" or "three of a kind'. The problem is, however, still very difficult for relational learning algorithms.

Consider the simple straight; it is five sequentially ranked cards, such as $\{5,6,7,8,9\}$, of mixed suits. This is difficult for a relational learner using the standard comparison operators $\{=,<,>,<=,>=, \neq\}$ because there is no recognition of the ranks between two cards. For example, a hand containing cards with ranks $\{5,7,8,9,10\}$ are five cards in ascending order, but there is a gap in the ranks so it is not a straight.

\section{Overloaded attribute values}

To make things more complicated, the Ace is always represented by the first index ' 1 ', but a straight can include an Ace at either the top of bottom of the rankings: $\{1$ (Ace), 2, $3,4,5\}$ or $\{10,11$ (Jack), 12 (Queen), 13 (King), 1 (Ace) $\}$. The problem could be reduced or eliminated by creating an additional symbol. The new symbol could represent Ace-High, while the existing symbol could represent Ace-Low. This would help, for example, in distinguishing between a straight flush and a royal flush. Although this 
would be a fair way to reduce complexity in this domain, it was not done for these experiments because it is adding knowledge to the system that may not be necessary.

Representation of the problem

The way in which instances of specific poker hands are represented is fully described below in section 6.2.4, Representation, starting on page 177.

\subsubsection{Statistics}

\begin{tabular}{|l|l|l|l|}
\hline Poker Hand & \# of hands & Probability & \# of combinations \\
\hline Royal Flush & 4 & 0.00000154 & 480 \\
\hline Straight Flush & 36 & 0.00001385 & 4320 \\
\hline Four of a kind & 624 & 0.0002401 & 74880 \\
\hline Full house & 3744 & 0.00144058 & 449280 \\
\hline Flush & 5108 & 0.0019654 & 612960 \\
\hline Straight & 10200 & 0.00392464 & 1224000 \\
\hline Three of a kind & 54912 & 0.02112845 & 6589440 \\
\hline Two pairs & 123552 & 0.04753902 & 14826240 \\
\hline One pair & 1098240 & 0.42256903 & 131788800 \\
\hline Nothing & 1302540 & 0.50117739 & 156304800 \\
\hline Total & $\mathbf{2 5 9 8 9 6 0}$ & $\mathbf{1 . 0}$ & $\mathbf{3 1 1 8 7 5 2 0 0}$ \\
\hline
\end{tabular}

Table 6.2: Poker hand odds

The above table contains information about the frequency of classes in the poker hand domain. The second column, number of hands, is the number of occurrences assuming that all semantically equivalent hands are removed. The total value is equal to [ 52 choose 5]. The probability represents the chance of randomly drawing the corresponding class given any five cards. The last column, number of combinations, is the number of hands that belong to the corresponding class. The total of this is equal to the permutation of five cards out of $52 ;(52 * 51 * 50 * 49 * 48)$. 
For an explanation on the probability of drawing any of the hands, please refer to Appendix D: Calculation of Poker Hand Statistics, starting on page 305.

It is easy to see that only a few classes represent the majority of the domain. Between the nothing and one pair hands, $92.37 \%$ of the dataset is accounted for.

\begin{tabular}{|l|l|l|l|}
\hline Poker Hand & \# of hands & Probability & \# of combinations \\
\hline Ace high straight & 1020 & 0.00039246 & 122400 \\
\hline Other straights & 9180 & 0.00353218 & 1101600 \\
\hline Total & $\mathbf{1 0 2 0 0}$ & $\mathbf{0 . 0 0 3 9 2 4 6 4}$ & $\mathbf{1 2 2 4 0 0 0}$ \\
\hline
\end{tabular}

Table 6.3: Breakdown of straight hands

Although straight hands are normally grouped together, the two categories are broken down because the Ace can make a straight as the first card $\{$ Ace, 2, 3, 4, 5\} or the last card \{Ten, Jack, Queen, King, Ace\}. The category with the Ace in the top position is noted because it is the non-normal case for this dataset.

In the set of other straights the span of ranks is always five. The Ace high straight is different because the actual ranks are $\{1,10,11,12,13\}$, where the span is exactly 13 . For comparison purposes is it interesting to note that a royal flush is always an Ace high straight, while a straight flush is always in the other straight category. 


\subsubsection{Representation}

Each instance consists of five cards that are not sorted by suit or rank, followed by the class. There are a total of ten predictive attributes that describe the five cards, as follows: \{Suit of card \#1, Rank of card \#1, .., Suit of card \#5, Rank of card \#5\}.

For maximum compatibility between algorithms, the attributes in this dataset can be represented in ordinal or nominal form. For algorithms that can take advantage of nominal values it is best to specify the suits as such, because it makes no sense to compare them other than for equality. The opposite is true for ranks, where it can be useful to know that certain cards have a higher or lower rank than other cards.

Ranks 1-5: $\{1$ (Ace), 2, 3, 4, 5, 6, 7, 8, 9, 10, 11 (Jack), 12 (Queen), 13 (King) $\}$

Suits 1-5: $\{1$ (Hearts), 2 (Spades), 3 (Diamonds), 4 (Clubs) $\}$

Class: 0-9

Examples

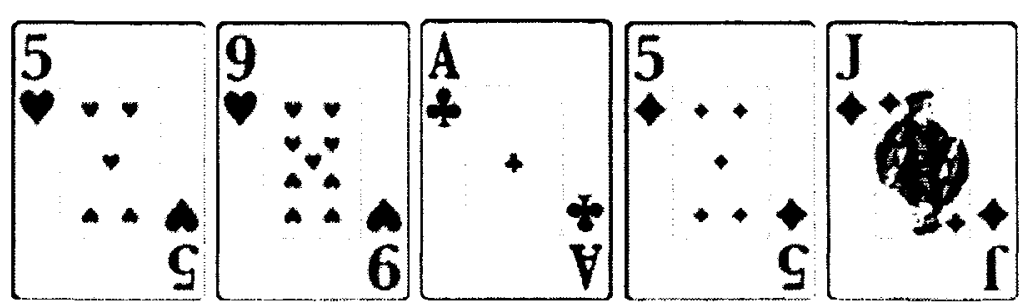

$1,5,1,9,4,1,3,5,3,11,1(5 \mathrm{H}, 9 \mathrm{H}, \mathrm{AC}, 5 \mathrm{D}, \mathrm{JD}=$ pair $)$ 


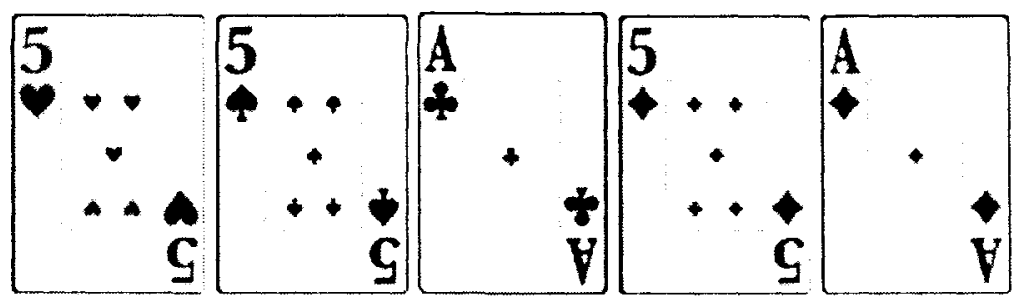

$1,5,2,5,4,1,3,5,3,1,6(5 \mathrm{H}, 5 \mathrm{~S}, \mathrm{AC}, 5 \mathrm{D}, \mathrm{AD}=$ full house $)$

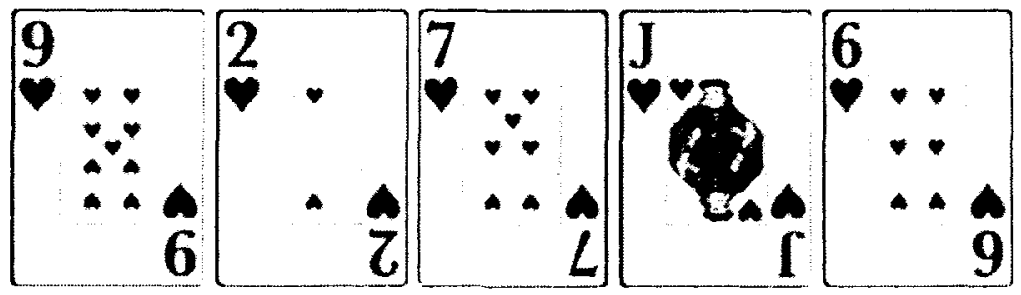

$1,9,1,2,1,7,1,11,1,6,5(9 \mathrm{H}, 2 \mathrm{H}, 7 \mathrm{H}, \mathrm{JH}, 6 \mathrm{H}=$ flush $)$

Optionally an algorithm can sort these cards to reduce the search space, but the problem is that it might produce rules that are dependant on the card hands always being sorted. In the spirit of comparing algorithms in a fair way it is important that no rules of poker, or even of playing cards, are relied upon. In the most general terms this means that the algorithm is incapable of sorting because it does not know that a single card is represented by a pair of values.

Another drawback to sorting cards, perhaps in a directed pre-processing stage, is that a large number of instances could be lost. By sorting and removing duplicates it effectively removes data points, and although this reduces the search space it also removes examples that could guide the learning algorithm in a positive way. 


\subsection{Supervised versus Unsupervised}

\section{Supervised}

The most obvious supervised problem is the classification of poker hands as described above, but it is possible to perform other classification tasks such as:

1: Classify the hand as Lose (nothing) versus Win (Any class other than nothing). If an additional attribute reflecting this were added to the dataset then less pre-processing would be required for algorithms that can only handle binary classification tasks.

2: Based on the cards, determine whether or not the hand is complete as opposed to perhaps being improved if one or more cards are discarded and replaced. The actual cards to be discarded do not have to be considered. For example: a pair has room for improvement, while a flush does not because all five cards are required.

A more interesting and interactive supervised task would be to have the computer decide which cards to hold after being dealt five cards. A model can be built using a large number training rounds that might include the following steps:

1) Computer receives five cards.

2) Computer can discard $0-5$ cards of choosing.

3) The discarded cards are replaced randomly from the same deck, and cannot contain a card previously discarded from the current training round.

4) The hand is scored and then paid out if it wins. See payout details below.

5) Record the amount of the payout. 
Instead of using the poker hand class information, include the payout in terms of odds. Typically a pair pays 1-1, while a royal flush pays 250-1. Based on the payout received the algorithm could learn what makes a "good" hand, and learn which cards to discard such that the likelihood of higher payout is maximized in the long run. This can also be simplified to the task of winning versus losing, where the amount of the payout is irrelevant.

\section{$\underline{\text { Unsupervised }}$}

An unsupervised learning task for the poker hand dataset would normally require the removal of the class attribute. Using only the predictive attributes:

1: Try to partition or cluster the records. Depending on the algorithm it might split the set along the lines of the poker hand classes, or perhaps find other regularities among ranks or suits that are not obvious to people familiar with poker.

2: Generate association rules to find out what occurs in the same hand. For example, depending on the specified confidence and support, it might discover that at most four cards with the same rank can appear in any single instance.

\subsection{Mapping to Real-World Problem Domains}

The poker hand classification task is similar to the problem of resource allocation.

Consider the following example: 
A computer is in charge of allocating resources for tasks received from departments within an organization. There are a finite number of resources available to complete tasks as they are received. There are several types of resources, and each type can have one or more available for use. In this example the resource pool contains: $\{20$ computers, 3 printers, 1 plotter, 2 technical support people, 8 data entry people, 1 meeting room\}.

Different tasks require different resources, and therefore different combinations of available resources correspond to tasks that can currently be accepted. Tasks are scored according to how much, and what types of resources they require. The scores, which are really a measure of resource demand, become the class attribute in the dataset.

The similarities that can be drawn to the poker hand dataset relate to matching tasks with poker hands. The cards in the deck can be thought of as resources, where there are a finite number of cards available, and they can be divided into different types. Specific resources are similar to concepts that can be described by card rank or card suit. Certain combinations of resources are required to complete certain tasks, much in the same way that certain combinations of cards are required to make certain hands.

\section{Example}

One goal for a learning algorithm might be to determine what tasks can be completed given a list of available resources.

Task A: 20 hours of data entry time on a specific project. Score: $\mathbf{5 0 0}$ 
Task B: Printing of system documentation: Score: 20

The Score represents the amount of effort required, and different combinations of required resources yield different scores. Resources that are scarce have a higher score, which links the score to how available a certain resource is. A parallel can be drawn between resource intensive tasks and rarely occurring poker hands.

Because tasks can be run concurrently, and presumably the desire will be to maximize the number of tasks being handled, only a subset of the resources in the pool will be available at any one time. It would be important to have a list of what tasks can be completed given this constraint. In terms of a binary classification task the question could simply be: Does the system currently have resources available to run this task?

A similar constraint exists in the poker hand dataset. Specifically, as an example, only so many straight flush hands (very resource-intensive tasks) can be dealt (processed) simultaneously when there is only a single deck of cards (limited resource pool) available.

\subsection{Training and Testing Sets}

The Poker Hand dataset consists of two different training sets and a single test set. In all cases, either one of the training sets is used to build the predictive model. Regardless of which training set the model was built with, the same test set is always used for 
evaluating the model. The two training sets and the single test set are described in more detail below.

\section{True distribution training set}

The first set contains 25010 instances and is intended to be used for training with a true distribution of classes. The first 25000 instances were randomly generated, but did not contain any royal flush or straight flush hands. Despite the fact that adding hands artificially skews the odds, the following hands were created to give the missing classes some representation:

\begin{tabular}{|c|c|c|c|c|c|c|c|c|c|c|l|}
\hline S1 & C1 & S2 & C2 & S3 & C3 & S4 & C4 & S5 & C5 & Class & Name \\
\hline 1 & 10 & 1 & 11 & 1 & 13 & 1 & 12 & 1 & 1 & 9 & Royal Flush \\
\hline 2 & 11 & 2 & 13 & 2 & 10 & 2 & 12 & 2 & 1 & 9 & Royal Flush \\
\hline 3 & 12 & 3 & 11 & 3 & 13 & 3 & 10 & 3 & 1 & 9 & Royal Flush \\
\hline 4 & 10 & 4 & 11 & 4 & 1 & 4 & 13 & 4 & 12 & 9 & Royal Flush \\
\hline 4 & 1 & 4 & 13 & 4 & 12 & 4 & 11 & 4 & 10 & 9 & Royal Flush \\
\hline 1 & 2 & 1 & 4 & 1 & 5 & 1 & 3 & 1 & 6 & 8 & Straight Flush \\
\hline 1 & 9 & 1 & 12 & 1 & 10 & 1 & 11 & 1 & 13 & 8 & Straight Flush \\
\hline 2 & 1 & 2 & 2 & 2 & 3 & 2 & 4 & 2 & 5 & 8 & Straight Flush \\
\hline 3 & 5 & 3 & 6 & 3 & 9 & 3 & 7 & 3 & 8 & 8 & Straight Flush \\
\hline 4 & 1 & 4 & 4 & 4 & 2 & 4 & 3 & 4 & 5 & 8 & Straight Flush \\
\hline
\end{tabular}

Table 6.4: Manually added instances for poker hand training

In this table, $\mathbf{S} \#$ indicates the suit associated with the card in the specified position. The C\# indicates the rank of the card in the specified position. The name is here for information purposes only, and is not included in the dataset.

The statistics for the true distribution training data, including the manually added instances, is detailed in the following table: 


\begin{tabular}{|l|c|c|c|c|c|}
\hline \multicolumn{1}{|c|}{ Poker Hand } & $\#$ & Distribution & Probability & Difference & Ratio \\
\hline Royal flush & 5 & 0.00019992 & 0.00000154 & 0.00019838 & 129.8182 \\
\hline Straight flush & 5 & 0.00019992 & 0.00001385 & 0.00018607 & 14.43466 \\
\hline Four of a kind & 6 & 0.0002399 & 0.0002401 & $-1.9596 \mathrm{E}-07$ & 0.999184 \\
\hline Full house & 36 & 0.00143942 & 0.00144058 & $-1.1558 \mathrm{E}-06$ & 0.999198 \\
\hline Flush & 54 & 0.00215914 & 0.0019654 & 0.00019374 & 1.098573 \\
\hline Straight & 93 & 0.00371851 & 0.00392465 & -0.00020614 & 0.947476 \\
\hline Three of a kind & 513 & 0.0205118 & 0.02112845 & -0.00061665 & 0.970814 \\
\hline Two pairs & 1206 & 0.04822071 & 0.04753902 & 0.00068169 & 1.01434 \\
\hline One pair & 10599 & 0.42379048 & 0.42256903 & 0.00122145 & 1.002891 \\
\hline Nothing & 12493 & 0.49952019 & 0.50117739 & -0.0016572 & 0.996693 \\
\hline Total & $\mathbf{2 5 0 1 0}$ & $\mathbf{1}$ & $\mathbf{1}$ & & \\
\hline
\end{tabular}

Table 6.5: Statistics for the poker hand true distribution training set

The second column is the number of hands for the corresponding class contained in the dataset. The third column, distribution, is the probability of that class being found within the dataset. The fourth column, probability, is the value that is expected when the cards are drawn at random from the space of the entire domain. The fifth column, difference, shows the difference between the distribution and the probability for each class. Finally, the ratio points out how much more often these hands occur in the dataset as compared to being drawn randomly. For example, the royal flush is 129.8 times more likely to occur in this dataset as compared to being drawn randomly, while drawing a straight occurs less frequently than normal at $94.7 \%$.

\section{Uniform distribution training set}

The second set contains 4800 instances and is intended to be used for training with a uniform class distribution. Using a combination of over-sampling rare classes, and undersampling the more common classes, this dataset contains 480 instances of each of the 10 
classes. With only 480 possible royal flush hands, every possible instance in this class is represented.

The uniform dataset is intended to be used for training a model that will distinguish between classes, but not allow it to take advantage of the probability estimated by statistical calculations. Although over-sampling and under-sampling are only two of many techniques for dealing with unbalanced data sets, rules generated using this balance might have better performance where the sampling is not representative of the domain, or where the frequency of classes within the data changes over time.

The effects of changing the class representation within datasets by under-sampling or over-sampling are described in [Chawla et al., 2002; Drummond, Holte, 2003].

\begin{tabular}{|l|c|c|c|c|c|}
\hline \multicolumn{1}{|c|}{ Poker Hand } & $\#$ & Distribution & Probability & Difference & Ratio \\
\hline Royal flush & 480 & 0.1 & 0.00000154 & 0.09999846 & 64935.06 \\
\hline Straight flush & 480 & 0.1 & 0.00001385 & 0.09998615 & 7220.217 \\
\hline Four of a kind & 480 & 0.1 & 0.0002401 & 0.0997599 & 416.4931 \\
\hline Full house & 480 & 0.1 & 0.00144058 & 0.09855942 & 69.41649 \\
\hline Flush & 480 & 0.1 & 0.0019654 & 0.0980346 & 50.88023 \\
\hline Straight & 480 & 0.1 & 0.00392465 & 0.09607535 & 25.47998 \\
\hline Three of a kind & 480 & 0.1 & 0.02112845 & 0.07887155 & 4.732955 \\
\hline Two pairs & 480 & 0.1 & 0.04753902 & 0.05246098 & 2.103535 \\
\hline One pair & 480 & 0.1 & 0.42256903 & -0.32256903 & 0.236648 \\
\hline Nothing & 480 & 0.1 & 0.50117739 & -0.40117739 & 0.19953 \\
\hline Total & $\mathbf{4 8 0 0}$ & $\mathbf{1}$ & $\mathbf{1}$ & & \\
\hline
\end{tabular}

Table 6.6: Statistics for the poker hand uniform distribution training set

As can be seen by looking at the Ratio column, none of the classes are distributed very closely to their true probability. The class with nothing is largely under-represented, containing less than $20 \%$ of the instances expected for a dataset this size. Conversely the 
royal flush is over-represented to the point where it is almost 65000 times more likely to find a royal flush as compared to drawing one at random.

\section{$\underline{\text { Testing set }}$}

The last dataset contains one million instances and is designed for evaluating models created using either of the two specified training sets. This large number of records is required to have each class represented with more than one instance, but still maintain a distribution that is very close to the true probability.

\begin{tabular}{|l|c|c|c|c|c|}
\hline \multicolumn{1}{|c|}{ Poker Hand } & $\#$ & Distribution & Probability & Difference & Ratio \\
\hline Royal flush & 3 & 0.000003 & 0.00000154 & 0.00000146 & 1.948052 \\
\hline Straight flush & 12 & 0.000012 & 0.00001385 & -0.00000185 & 0.866426 \\
\hline Four of a kind & 230 & 0.00023 & 0.0002401 & -0.0000101 & 0.957934 \\
\hline Full house & 1424 & 0.001424 & 0.00144058 & -0.00001658 & 0.988491 \\
\hline Flush & 1996 & 0.001996 & 0.0019654 & 0.0000306 & 1.015569 \\
\hline Straight & 3885 & 0.003885 & 0.00392465 & -0.00003965 & 0.989897 \\
\hline Three of a kind & 21121 & 0.021121 & 0.02112845 & $-7.45 \mathrm{E}-06$ & 0.999647 \\
\hline Two pairs & 47622 & 0.047622 & 0.04753902 & $8.298 \mathrm{E}-05$ & 1.001746 \\
\hline One pair & 422498 & 0.422498 & 0.42256903 & $-7.103 \mathrm{E}-05$ & 0.999832 \\
\hline Nothing & 501209 & 0.501209 & 0.50117739 & $3.161 \mathrm{E}-05$ & 1.000063 \\
\hline Total & $\mathbf{1 0 0 0 0 0 0}$ & $\mathbf{1}$ & $\mathbf{1}$ & & \\
\hline
\end{tabular}

\section{Table 6.7: Statistics for the poker hand testing set}

\section{Exhaustive evaluation with every possible instance}

Although the entire space of the poker hand domain is bounded it is still very large. If questions about the predictive accuracy of any given model arise, then in theory this dataset could be created and the model evaluated. This dataset has not been generated because it is assumed that the one million instance testing set is very representative of the entire domain, and further testing instances are not required. 


\subsection{Classifying the Poker Hand Dataset}

For the purpose of determining how difficult this classification problem is, an empirical study was conducted using several algorithms available within the Waikato environment for knowledge analysis (Weka [Witten, Frank, 2005]).

For each experiment a model was created by training with the true distribution (25010 instances) dataset, as well as by training with the uniform (4800 instances) dataset. In both cases the models were evaluated by using them on the million instance test set.

\subsubsection{True Distribution Dataset}

The goal of a learning algorithm is to achieve better than $50.121 \%$, which is the percentage of instances that fall within the majority (nothing) class. An algorithm that has predictive accuracy lower than this will be worse than always choosing the majority, while values higher than this indicate that some correct predictions are being made.

\begin{tabular}{|l|l|l|}
\hline Algorithm & Correct & Incorrect \\
\hline Bagging & $48.755 \%$ & $51.245 \%$ \\
\hline BayesNet & $50.121 \%$ & $49.879 \%$ \\
\hline ConjunctiveRule & $50.121 \%$ & $49.879 \%$ \\
\hline FilteredClassifier & $50.121 \%$ & $49.879 \%$ \\
\hline J48 & $\mathbf{5 4 . 2 2 5 \%}$ & $45.775 \%$ \\
\hline JRip & $\mathbf{5 6 . 6 1 6} \%$ & $43.384 \%$ \\
\hline Logistic & $50.083 \%$ & $49.917 \%$ \\
\hline MultilayerPerceptron & $\mathbf{5 3 . 1 6 2} \%$ & $46.838 \%$ \\
\hline NaiveBayes & $50.090 \%$ & $49.910 \%$ \\
\hline RandomForest & $48.525 \%$ & $51.475 \%$ \\
\hline REPTree & $48.439 \%$ & $51.561 \%$ \\
\hline SMO & $47.495 \%$ & $52.505 \%$ \\
\hline DecisionStump & $50.121 \%$ & $49.879 \%$ \\
\hline VFI & $0.904 \%$ & $99.096 \%$ \\
\hline ZeroR & $50.121 \%$ & $49.879 \%$ \\
\hline
\end{tabular}

Table 6.8: Algorithm performance on model trained with the true distribution 
Values of predictive accuracy in the above table show that most of the learning algorithms that were evaluated had poor performance on the poker hand dataset. Five of the algorithms score the threshold accuracy of $50.121 \%$, which probably means they assume the majority class for most or all instances.

Several of the algorithms score below the assume-majority threshold, while only three score above. The best algorithm is JRip, which has a predictive accuracy of $56.616 \%$. The model contains 19 rules that cover instances from several classes, with the default class being nothing.

\section{Example JRip rules}

$(\mathrm{R} 1<=2)$ and $(\mathrm{R} 3>=8)$ and $(\mathrm{S} 3=3)$ and $(\mathrm{S} 1=3)$ and $(\mathrm{S} 5=3)$ and $(\mathrm{S} 2=3)$ and $(\mathrm{S} 4=$ $3) \Rightarrow$ Class $=5(5.0 / 0.0)$

$(\mathrm{R} 3<=5)$ and $(\mathrm{S} 3=4)$ and $(\mathrm{S} 5=4)$ and $(\mathrm{S} 1=4)$ and $(\mathrm{S} 2=4)$ and $(\mathrm{S} 4=4)=>$ Class $=5$

$(\mathrm{R} 1<=2)$ and $(\mathrm{R} 5<=2)$ and $(\mathrm{R} 3<=1)$ and $(\mathrm{R} 1<=1)$ and $(\mathrm{R} 5<=1) \Rightarrow$ Class $=3$

$$
\begin{aligned}
& (\mathrm{R} 3<=2) \text { and }(\mathrm{R} 1<=2)=>\text { Class }=1(318.0 / 137.0) \\
& \Rightarrow \text { Class }=0(16211.0 / 6526.0)
\end{aligned}
$$

The rules created by JRip are very specific because attribute relationships are not considered, although it still performs well in some respects. The first rule shown is an 
example of JRip trying to classify a flush. It first recognizes that each of the five attributes representing the card suits have to be the same, but is lacking generality because it only covers the value representing diamonds. It is apparent that JRip is trying to work around the straight flush and royal flush by imposing further restrictions on the card ranks, namely "(R1 <=2) and (R3 >=8)".

Using $[(\mathrm{R} 1<=2)$ and $(\mathrm{R} 3>=8)]$ as constraints will eliminate virtually all straight hands because the distance between the two is greater than the number of cards dealt in a hand, which means the cards cannot be ordered consecutively without a gap in ranks. The only problem arises in the case of a very specific royal flush, as follows:

R1 must be the Ace of diamonds, while R2, R3, R4, and R5 must be the Ten, Jack, Queen, and King of diamonds in no specific order. If these constraints hold then this rule will fail. Due to the rarity of this class in the training set, a counter-example was not available for the learning algorithm to recognize and avoid this problem.

The rules produced by JRip also contain redundant terms, such as in the third rule that is attempting to classify three of a kind. In the same rule the terms " $(\mathrm{R} 1<=2)$ " and "(R1<= 1)" appear, but it is obvious that the first is not only subsumed by the second, but also made invalid because the upper bound changes.

Overall it seems that lack of generality expressed by the rules created by JRip leads to a fragile model that ultimately does very poorly on unseen data. 
Also of interest in this experiment is the REPTree algorithm, which did not answer the abundant classes as well as other algorithms, but was able to successfully determine what a flush hand is. The flush class was covered $100 \%$, although there were errors because the straight flush and royal flush instances were also classified as a regular flush.

\subsubsection{Uniform Distribution Dataset}

Without the benefit of being able to calculate the majority class, the worst that the algorithm should do is random. A completely random choice will be correct one in ten times, which means that the goal of the algorithm is to achieve better than $10 \%$.

\begin{tabular}{|l|c|c|}
\hline Algorithm & Correct & Incorrect \\
\hline Bagging & $\mathbf{1 7 . 8 3 6} \%$ & $82.164 \%$ \\
\hline BayesNet & $8.778 \%$ & $91.223 \%$ \\
\hline ConjunctiveRule & $0.001 \%$ & $99.999 \%$ \\
\hline FilteredClassifier & $\mathbf{1 4 . 0 3 7 \%}$ & $\mathbf{8 5 . 9 6 3 \%}$ \\
\hline J48 & $\mathbf{1 6 . 0 2 0} \%$ & $83.980 \%$ \\
\hline JRip & $1.585 \%$ & $98.415 \%$ \\
\hline Logistic & $9.956 \%$ & $90.044 \%$ \\
\hline MultilayerPerceptron & $\mathbf{1 9 . 5 3 8} \%$ & $80.463 \%$ \\
\hline NaiveBayes & $\mathbf{1 0 . 4 0 9 \%}$ & $89.591 \%$ \\
\hline RandomForest & $\mathbf{1 7 . 2 0 0 \%}$ & $82.800 \%$ \\
\hline REPTree & $\mathbf{1 5 . 4 6 7 \%}$ & $84.533 \%$ \\
\hline SMO & $\mathbf{1 7 . 5 9 8} \%$ & $82.402 \%$ \\
\hline DecisionStump & $0.001 \%$ & $99.999 \%$ \\
\hline VFI & $\mathbf{1 5 . 7 0 6 \%}$ & $84.294 \%$ \\
\hline ZeroR & $\mathbf{5 0 . 1 2 1} \%$ & $49.879 \%$ \\
\hline Tab
\end{tabular}

Table 6.9: Algorithm performance on model trained with the uniform distribution

Most of the algorithms did achieve results that are better than random, however none, with the apparent exception of ZeroR, were able to achieve more than $20 \%$ accuracy. 
Although the ZeroR algorithm appeared to perform significantly better than the others when training on the uniform dataset, this is not really the case. The reason for the betterthan-average performance is that it simply created a rule that classified every element as the first class. Coincidentally, this was the nothing class. This means that when training it achieves exactly $10 \%$, and when evaluating using the million-instance test set it correctly classified all of the nothing hands (50.121\%) and was incorrect on everything else.

Consequently, the ZeroR algorithm should not be considered significantly better than the others.

The next best algorithm, MultilayerPerceptron, achieved an accuracy of $19.538 \%$, but does not have anything noteworthy in the results.

The JRip algorithm is interesting because it was the best performer when the model was trained using the true distribution dataset, and one of the worst performers when it was trained using the uniform set. Looking at the results more closely revealed that training on the uniform dataset lead the algorithm to assume that most instances belonged to the royal flush class. Although it correctly classified all of the royal flush instances, many of the other classes were misclassified as such.

The only learning algorithm to achieve better accuracy on the uniform dataset was VFI, with $15.706 \%$ on the uniform data as compared to $0.904 \%$ on the representative data. As VFI depends on votes based on feature values, this increase is most likely observed 
because additional data points made available through over-sampling are considered during learning.

\subsubsection{Summary of Weka experiments}

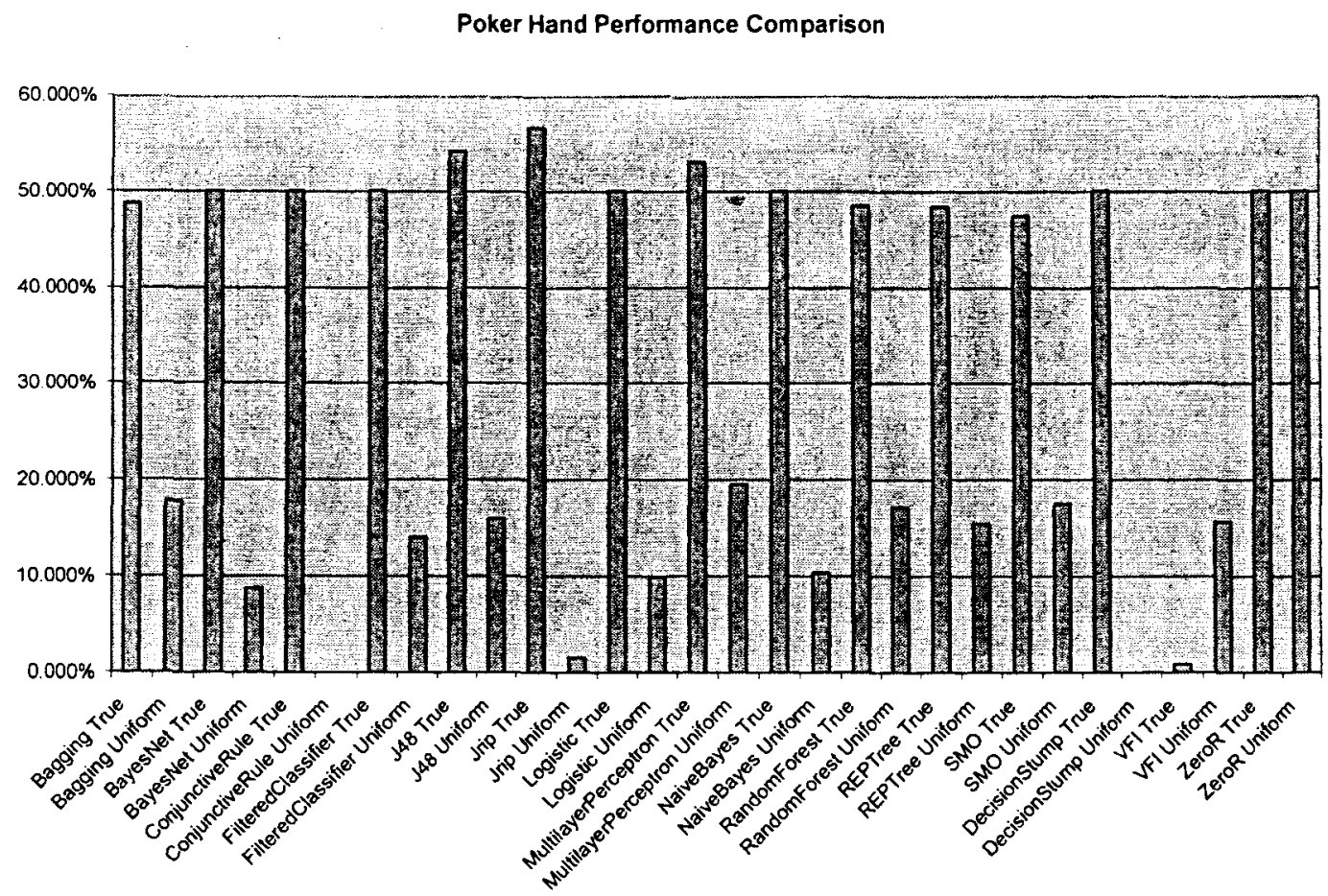

Figure 6.1: Summary of results achieved using Weka

With respect to creating an accurate predictive model, the poker hand dataset was a difficult problem for all of the learning algorithms that were evaluated. Most of the algorithms performed worse when training on the uniform dataset. 




Figure 6.2: Class coverage for the JRip algorithm on the Poker Hand dataset

The best algorithm, JRip, is a rule discovery algorithm. JRip did not correctly classify any of the classes with $100 \%$ accuracy. It was, however, able to classify three different classes with more than $10 \%$ accuracy.

\subsection{Using EvRFind for Rule Discovery}

\subsubsection{Initial experiments}

The poker hand dataset has been used to test and evaluate different features within EvRFind and in doing so a large number of experiments have been run. Before the development of Gen-Functions there were results recorded for 240 experiments, including many different combinations of parameter settings using both of the training sets. The summary of these results when evaluated using the million-instance test set is described by the following two tables: 


\begin{tabular}{|l|c|c|c|c|}
\hline Dataset & $\begin{array}{c}\text { Max } \\
\text { rules }\end{array}$ & $\begin{array}{c}\text { Overall } \\
\text { average } \\
\text { accuracy }\end{array}$ & $\begin{array}{c}\text { Best } \\
\text { accuracy }\end{array}$ & $\begin{array}{c}\text { Average } \\
\text { missed } \\
\text { instances }\end{array}$ \\
\hline Poker Hands - Uniform distribution & 500 & $28.05 \%$ & $33.18 \%$ & $35.42 \%$ \\
\hline Poker Hands - Uniform distribution & 2500 & $43.81 \%$ & $47.83 \%$ & Nil \\
\hline
\end{tabular}

Table 6.10: Initial Poker Hand experiments with EvRFind - Uniform distribution

\begin{tabular}{|l|c|c|c|c|}
\hline Dataset & $\begin{array}{c}\text { Max } \\
\text { rules }\end{array}$ & $\begin{array}{c}\text { Overall } \\
\text { average } \\
\text { accuracy }\end{array}$ & $\begin{array}{c}\text { Best } \\
\text { accuracy }\end{array}$ & $\begin{array}{c}\text { Average } \\
\text { missed } \\
\text { instances }\end{array}$ \\
\hline Poker Hands - True distribution & 500 & $58.63 \%$ & $62.47 \%$ & $29.53 \%$ \\
\hline Poker Hands - True distribution & 2500 & $92.78 \%$ & $93.23 \%$ & $0.03 \%$ \\
\hline
\end{tabular}

Table 6.11: Initial Poker Hand experiments with EvRFind - True distribution

The max rules column is the maximum number of rules that EvRFind was allowed to work with during any particular experiment. The best accuracy used the combination of parameters that achieved the best 10-run accuracy for the experiment, and this combination of options eventually became the default configuration for EvRFind.

As can be seen in the first table, EvRFind performed similarly to other learning algorithms when training on the uniformly distributed dataset. EvRFind did not have a default-class, nor did it give preference to classes that were more likely to appear in the dataset, so a more extensive analysis of the rules was done.

Example: 500 rule population training on uniform dataset

In one experiment, allowing EvRFind to use up to 500 rules, the following were the top seven: 


\begin{tabular}{|c|l|c|}
\hline Confidence & Terms in the Antecedent & Class \\
\hline $94.55 \%$ & $(\mathrm{C} 1<\mathrm{C} 5) \wedge(\mathrm{S} 3=\mathrm{S} 5) \wedge(\mathrm{C} 1<\mathrm{C} 2) \wedge(\mathrm{S} 5=\mathrm{S} 1) \wedge(\mathrm{C} 5<9) \wedge(\mathrm{S} 5=\mathrm{S} 2) \wedge(\mathrm{C} 4>\mathrm{C} 1) \wedge(\mathrm{C} 2<\mathrm{C} 5)$ & 8 \\
\hline $100.00 \%$ & $(\mathrm{C} 2 !=\mathrm{C} 1) \wedge(\mathrm{C} 4=\mathrm{C} 2) \wedge(\mathrm{C} 5=\mathrm{C} 4) \wedge(\mathrm{C} 5=\mathrm{C} 3)$ & 7 \\
\hline $100.00 \%$ & $(\mathrm{C} 5=\mathrm{C} 1) \wedge(\mathrm{C} 5=\mathrm{C} 4) \wedge(\mathrm{C} 5=\mathrm{C} 3)$ & 7 \\
\hline $100.00 \%$ & $(\mathrm{C} 2=\mathrm{C} 3) \wedge(\mathrm{C} 2=\mathrm{C} 5) \wedge(\mathrm{S} 2 !=\mathrm{S} 1) \wedge(\mathrm{S} 4 !=\mathrm{S} 5) \wedge(\mathrm{C} 3=\mathrm{C} 1)$ & 7 \\
\hline $100.00 \%$ & $(\mathrm{C} 5=\mathrm{C} 2) \wedge(\mathrm{C} 1=\mathrm{C} 3) \wedge(\mathrm{C} 2 !=7) \wedge(\mathrm{S} 1 !=2) \wedge(\mathrm{Cl}=\mathrm{C} 4)$ & 6 \\
\hline $100.00 \%$ & $(\mathrm{C} 2 !=\mathrm{C} 1) \wedge(\mathrm{C} 4=\mathrm{C} 2) \wedge(\mathrm{Cl}=\mathrm{C} 3) \wedge(\mathrm{Cl}=\mathrm{C} 5)$ & 6 \\
\hline $100.00 \%$ & $(\mathrm{C} 2=12) \wedge(\mathrm{C} 3=13) \wedge(\mathrm{S} 5=\mathrm{S} 4) \wedge(\mathrm{S} 3=\mathrm{S} 1) \wedge(\mathrm{C} 5>8) \wedge(\mathrm{S} 1=4)$ & 9 \\
\hline
\end{tabular}

\section{Table 6.12: Excerpt from EvRFind hierarchy - initial experiments}

On the testing set the 500-rule hierarchy that included the rules above achieved $22.92 \%$ accuracy. The entire experiment that included 10 runs with different random number seeds averaged $25.22 \%$ accuracy overall. The number of missed records is 452690 (418942.2 is the 10 -run average).

Example: 2500 rule population training on true distribution dataset

It was determined that the nothing and pair classes contained so many more example instances in the true distribution dataset that EvRFind was simply better able to discover and verify rules. This meant that although $90+\%$ accuracy was achieved, many of the 2500 rules allowed during the experiment were active and each was used to classify very small numbers of instances. In the worst cases, some rules were used to address single examples. Rules of this type are not useful because given enough of them it is possible to achieve $100 \%$ accuracy trivially by creating one rule for each instance in the dataset [Freitas, 2002b].

During the analysis of these results it was also found that many classes were being covered incompletely, and the reason becomes obvious when considering, for example, the minimum number of rules required to classify two pairs. 
Example of minimum rules required to classify two pairs

If $(\mathrm{C} 1=\mathrm{C} 2) \wedge(\mathrm{C} 3=\mathrm{C} 4) \wedge(\mathrm{C} 1 !=\mathrm{C} 3) \wedge(\mathrm{C} 1 !=\mathrm{C} 5) \wedge(\mathrm{C} 3 !=\mathrm{C} 5)$ then $(\mathrm{CLASS}=2)$

If $(\mathrm{C} 1=\mathrm{C} 2) \wedge(\mathrm{C} 3=\mathrm{C} 5) \wedge(\mathrm{C} 1 !=\mathrm{C} 3) \wedge(\mathrm{C} 1 !=\mathrm{C} 4) \wedge(\mathrm{C} 3 !=\mathrm{C} 4)$ then $(\mathrm{CLASS}=2)$

If $(\mathrm{C} 1=\mathrm{C} 2) \wedge(\mathrm{C} 4=\mathrm{C} 5) \wedge(\mathrm{C} 1 !=\mathrm{C} 3) \wedge(\mathrm{C} 1 !=\mathrm{C} 4) \wedge(\mathrm{C} 3 !=\mathrm{C} 4)$ then $(\mathrm{CLASS}=2)$

If $(\mathrm{C} 1=\mathrm{C} 3) \wedge(\mathrm{C} 2=\mathrm{C} 4) \wedge(\mathrm{C} 1 !=\mathrm{C} 2) \wedge(\mathrm{C} 1 !=\mathrm{C} 5) \wedge(\mathrm{C} 2 !=\mathrm{C} 5)$ then $(\mathrm{CLASS}=2)$

If $(\mathrm{C} 1=\mathrm{C} 3) \wedge(\mathrm{C} 2=\mathrm{C} 5) \wedge(\mathrm{C} 1 !=\mathrm{C} 2) \wedge(\mathrm{C} 1 !=\mathrm{C} 4) \wedge(\mathrm{C} 2 !=\mathrm{C} 4)$ then $(\mathrm{CLASS}=2)$

If $(\mathrm{C} 1=\mathrm{C} 3) \wedge(\mathrm{C} 4=\mathrm{C} 5) \wedge(\mathrm{C} 1 !=\mathrm{C} 2) \wedge(\mathrm{C} 1 !=\mathrm{C} 4) \wedge(\mathrm{C} 2 !=\mathrm{C} 4)$ then $(\mathrm{CLASS}=2)$

If $(\mathrm{C} 1=\mathrm{C} 4) \wedge(\mathrm{C} 2=\mathrm{C} 3) \wedge(\mathrm{C} 1 !=\mathrm{C} 2) \wedge(\mathrm{C} 1 !=\mathrm{C} 5) \wedge(\mathrm{C} 2 !=\mathrm{C} 5)$ then $(\mathrm{CLASS}=2)$

If $(\mathrm{C} 1=\mathrm{C} 4) \wedge(\mathrm{C} 2=\mathrm{C} 5) \wedge(\mathrm{C} 1 !=\mathrm{C} 2) \wedge(\mathrm{C} 1 !=\mathrm{C} 3) \wedge(\mathrm{C} 2 !=\mathrm{C} 3)$ then $(\mathrm{CLASS}=2)$

If $(\mathrm{C} 1=\mathrm{C} 4) \wedge(\mathrm{C} 3=\mathrm{C} 5) \wedge(\mathrm{C} 1 !=\mathrm{C} 2) \wedge(\mathrm{C} 1 !=\mathrm{C} 3) \wedge(\mathrm{C} 2 !=\mathrm{C} 3)$ then $(\mathrm{CLASS}=2)$

If $(\mathrm{C} 1=\mathrm{C} 5) \wedge(\mathrm{C} 2=\mathrm{C} 3) \wedge(\mathrm{C} 1 !=\mathrm{C} 2) \wedge(\mathrm{C} 1 !=\mathrm{C} 4) \wedge(\mathrm{C} 2 !=\mathrm{C} 4)$ then $(\mathrm{CLASS}=2)$

If $(\mathrm{C} 1=\mathrm{C} 5) \wedge(\mathrm{C} 2=\mathrm{C} 4) \wedge(\mathrm{C} 1 !=\mathrm{C} 2) \wedge(\mathrm{C} 1 !=\mathrm{C} 3) \wedge(\mathrm{C} 2 !=\mathrm{C} 3)$ then $(\mathrm{CLASS}=2)$

If $(\mathrm{C} 1=\mathrm{C} 5) \wedge(\mathrm{C} 3=\mathrm{C} 4) \wedge(\mathrm{C} 1 !=\mathrm{C} 2) \wedge(\mathrm{C} 1 !=\mathrm{C} 3) \wedge(\mathrm{C} 2 !=\mathrm{C} 3)$ then $(\mathrm{CLASS}=2)$

If $(\mathrm{C} 2=\mathrm{C} 3) \wedge(\mathrm{C} 4=\mathrm{C} 5) \wedge(\mathrm{C} 1 !=\mathrm{C} 2) \wedge(\mathrm{C} 1 !=\mathrm{C} 4) \wedge(\mathrm{C} 2 !=\mathrm{C} 4)$ then $(\mathrm{CLASS}=2)$

If $(\mathrm{C} 2=\mathrm{C} 4) \wedge(\mathrm{C} 3=\mathrm{C} 5) \wedge(\mathrm{C} 1 !=\mathrm{C} 2) \wedge(\mathrm{C} 1 !=\mathrm{C} 3) \wedge(\mathrm{C} 2 !=\mathrm{C} 3)$ then $(\mathrm{CLASS}=2)$

If $(\mathrm{C} 2=\mathrm{C} 5) \wedge(\mathrm{C} 3=\mathrm{C} 4) \wedge(\mathrm{C} 1 !=\mathrm{C} 2) \wedge(\mathrm{C} 1 !=\mathrm{C} 3) \wedge(\mathrm{C} 2 !=\mathrm{C} 3)$ then $(\mathrm{CLASS}=2)$

There are many semantically equivalent rules for each of the above, and a different set could be used with overlapping instances, but this is an example of a minimally sized set that will accurately cover all of class \#2. The complexity and specificity of each rule ensures that full house and four-of-a-kind hands are not misclassified as two pairs. 
Additional examples of minimum rule sets can be found in Appendix $F$ : Examples of minimal rule sets, starting on page 310 .

In none of the original experiments was EvRFind able to successfully discover all of the above rules, nor any set of rules that could answer every instance of two pairs with $100 \%$ confidence. Examination of the results in some of these experiments revealed a very high number of low support rules. The following two rules, ranked \#9 and \#10 in the partial hierarchy shown earlier, are examples of this.

$[\mathrm{C} 100.00 \%, \mathrm{~S} 0.04 \%, \mathrm{R}=2, \mathrm{~W}=0]$

If $(\mathrm{C} 3=13) \wedge(\mathrm{S} 2 !=\mathrm{S} 3) \wedge(\mathrm{C} 1=10) \wedge(\mathrm{S} 1 !=\mathrm{S} 5) \wedge(\mathrm{S} 2=3)$ then $(\mathrm{CLASS}=2)$

$[\mathrm{C} 100.00 \%, \mathrm{~S} 0.02 \%, \mathrm{R}=1, \mathrm{~W}=0]$

If $(\mathrm{S} 5=\mathrm{S} 3) \wedge(\mathrm{C} 1=12) \wedge(\mathrm{C} 2=11) \wedge(\mathrm{C} 4=\mathrm{C} 3)$ then $(\mathrm{CLASS}=2)$

C $=$ Confidence,$S=$ Support, $\mathbf{R}=$ right, $\mathrm{W}=$ wrong

These two rules are so specific that they answer just three instances between the two of them, and it was obvious that more general concepts had to be discovered before this could improve. 


\subsubsection{Experiments with EvRFind including Gen-Functions}

The introduction of Gen-Functions into EvRFind allows for more abstract concepts to be discovered. A total of eighty experiments were run. The first forty were performed while training on the uniform dataset, and evaluating the results using the million-instance dataset. The second half of the experiments trained using the true-distribution data, and were also evaluated using the same million-instance dataset.

The maximum number of rules allowed was less than in previous experiments because it was expected that automatic generalization would reduce the requirements. A higher population allows for more rules to address a single concept, so by lowering the limit it handicaps the evolutionary process.

Several of the experiments were run twice in order to evaluate the hierarchy-protectedfitness option. In the result-tables shown in the next section, experiments are labeled with either HPF or Non-HPF to indicate whether the option was used during training. The results of this experimental evaluation is summarized later in this chapter.

\subsubsection{Overall Results - Uniform versus True Distribution}

\begin{tabular}{|l|c|c|c|c|}
\hline Options & $\begin{array}{c}\text { Max } \\
\text { rules }\end{array}$ & $\begin{array}{c}\text { Average } \\
\text { accuracy }\end{array}$ & $\begin{array}{c}\text { Best } \\
\text { accuracy }\end{array}$ & $\begin{array}{c}\text { Average } \\
\text { missed }\end{array}$ \\
\hline Poker Hands - Uniform - HPF & 100 & $68.84 \%$ & $86.69 \%$ & $0.2 \%$ \\
\hline Poker Hands - Uniform - Non-HPF & 100 & $71.89 \%$ & $90.15 \%$ & $0.15 \%$ \\
\hline Poker Hands - Uniform - HPF & 500 & $75.07 \%$ & $90.68 \%$ & $\mathrm{Nil}$ \\
\hline Poker Hands - Uniform - Non-HPF & 500 & $80.79 \%$ & $96.70 \%$ & Nil \\
\hline
\end{tabular}

Table 6.13: Poker Hand experiments with Gen-Functions - Uniform distribution 
The first entry in the table summarizes ten experiments that were trained on the uniform dataset using a rule population size of 100 . Although the average is not very good, at $68.84 \%$ it is still better than previous best of $43.81 \%$ that was trained on the same dataset but using a population size of 2500 rules. Also worth noting is the number of missed instances. As compared to the pre-Gen-Function experiments, most instances are addressed by at least one rule.

\begin{tabular}{|l|c|c|c|c|}
\hline Dataset & $\begin{array}{c}\text { Max } \\
\text { rules }\end{array}$ & $\begin{array}{c}\text { Average } \\
\text { accuracy }\end{array}$ & $\begin{array}{c}\text { Best } \\
\text { accuracy }\end{array}$ & $\begin{array}{c}\text { Average } \\
\text { missed }\end{array}$ \\
\hline Poker Hands - True - HPF & 100 & $96.76 \%$ & $99.25 \%$ & $0.1 \%$ \\
\hline Poker Hands - True - Non-HPF & 100 & $99.53 \%$ & $99.63 \%$ & Nil \\
\hline Poker Hands - True - HPF & 500 & $98.22 \%$ & $99.26 \%$ & Nil \\
\hline Poker Hands - True - Non-HPF & 500 & $99.69 \%$ & $99.96 \%$ & Nil \\
\hline
\end{tabular}

Table 6.14: Poker Hand experiments with Gen-Functions - True distribution

The second table contains results for the experiments that were run using the true distribution dataset. As can be seen when comparing the values for accuracy against those from the previous table, the true distribution dataset performs much better on average.

The best accuracy out of all the experiments that were run was $99.96 \%$, where a total of 999,557 out of $1,000,000$ instances were correctly classified. 


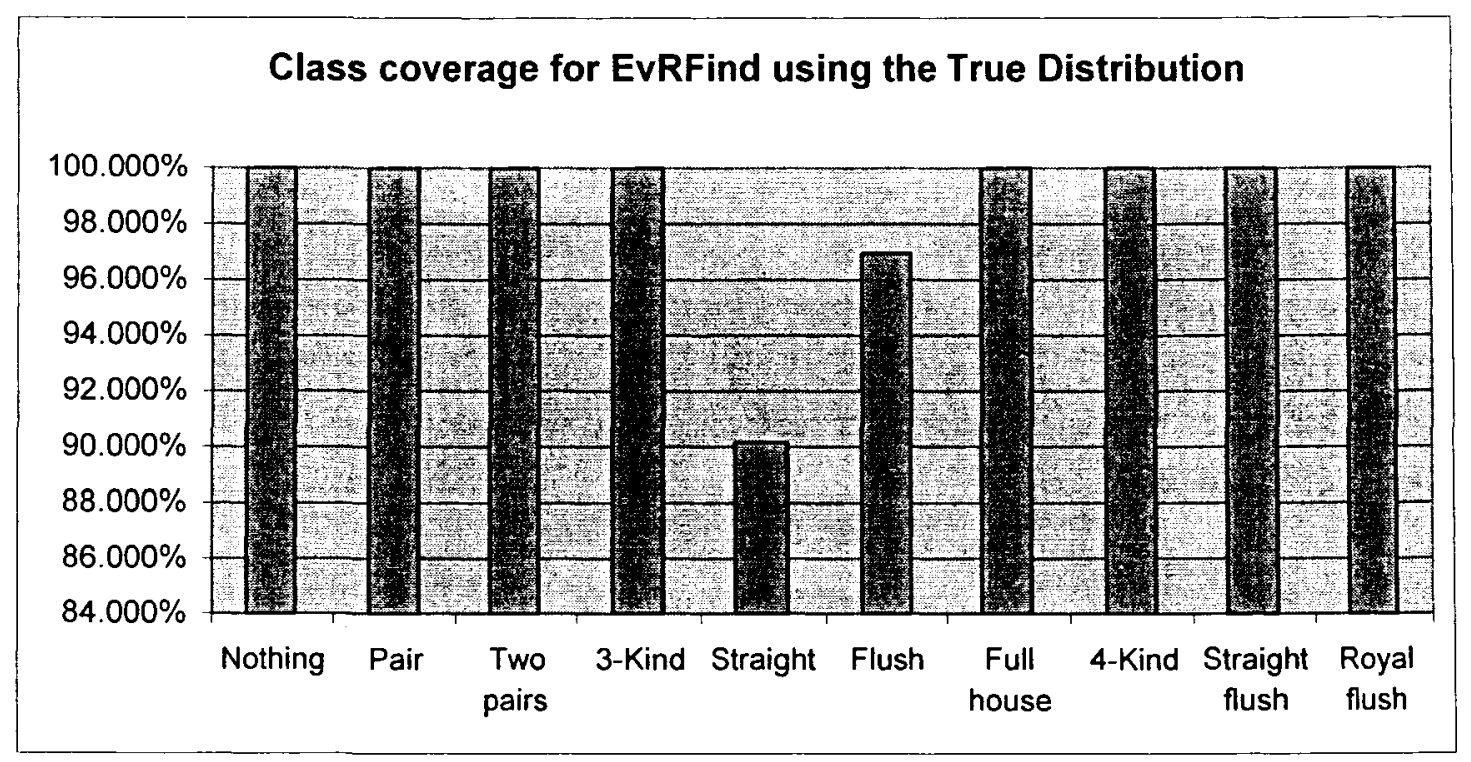

Figure 6.3: Class coverage for EvRFind in the best results

\subsubsection{Evaluating the Hierarchy Protected Fitness option}

The concept of the hierarchy-protected-fitness is described in Chapter 5.5.6: Building the hierarchy, starting on page 92 . The goal of experimenting with this option was to determine whether it would be more beneficial to direct the system towards creating a default hierarchy, or simply allow it to self-organize. The poker hand dataset was the first domain that was used to evaluate this option.

As can be seen in the result-tables in the previous section, the models that were trained using the HPF option did not perform as well as those that were trained without it. These preliminary results suggest that steering EvRFind towards creating a default hierarchy has a negative impact on the results, and therefore should not be done. 
An in-depth analysis of the results revealed several details about using the HPF option in the poker hand domain:

a) The search time was often reduced, especially in cases where the population size was large. This does not necessarily mean that the search ran for less time, but rather that the final solutions were found in earlier generations.

b) In most cases the predictive accuracy of the training set was higher.

c) The average predictive accuracy on the test set was always lower.

d) The number of missed instances on the test set was always higher.

These observations tend to indicate that the HPF option acted greedily in terms of exploring the domain and refining solutions. This had the effect of over-fitting the hierarchy, which is one of the very problems that the default hierarchy was designed to address.

Although it can be concluded that the HPF option should not be used to classify poker hands, there is still the possibility that it may be beneficial in other domains. More experimental evaluation of this option is described in Chapter 7: Miscellaneous Dataset Experiments, starting on page 228.

\subsubsection{Discovery of Concepts within the dataset}

Further analysis of the results revealed that different concepts were discovered in different experiments on the same data. This is as expected due to the probabilistic nature 
of the process. In the case where experiments used smaller populations, this was more prevalent because there is not as much diversity, and therefore a smaller percentage of the domain is explored during each generation.

\subsubsection{Concept of two pairs}

The first such higher-level concept discovered was for the classification of two pairs. It is comprised of a single Gen-Function accompanied by a single rule that makes use of it, as follows:

GF: $\$ A$ NEV(class $=$ Rank $)=2\{$ Variable $\}]$

$[\mathrm{C} 100.00 \%, \mathrm{~S} 10.00 \%, \mathrm{R}=480, \mathrm{~W}=0]$ : If $(\$ \mathrm{~A}=2)$ then $(\mathrm{CLASS}=2)$

The Gen-Function, $\$ \mathrm{~A}$, can be easily understood by looking at the components:

1) NEV; This function looks for the "number of equal values".

2) (class = Rank); The scope is restricted to the attributes that were labeled as "Rank" in the session definition.

3) $=2$; Specifies that the function is searching for cases where exactly two equal values are found. Subsets of cards, such as a pair that contributes to a three of $a$ kind, are not counted.

\section{Examples}

$(\$ A=0)$; There are no pairs of equal ranks. $\{3,5,7,2,9\}$

$(\$ \mathrm{~A}=1)$; There is exactly one pair of equal ranks. $\{\underline{\mathbf{3}}, 5, \underline{\mathbf{3}}, 2,9\}$ 
$(\$ \mathrm{~A}=2) ;$ There are two pairs of equal ranks. $(\underline{\mathbf{3}}, \underline{\mathbf{5}}, \underline{\mathbf{3}}, 2, \underline{\mathbf{5}}\}$

$(\$ A=3)$; There are three pairs of equal ranks, which is not possible in five cards.

With the definition of $\$ \mathrm{~A}$ as it is explained above, the rule translates to:

"If within the ranks of the five cards there are exactly two distinct cases where there are two equally ranked cards, then the hand is classified as two pairs".

This Gen-Function and rule combination was discovered while training on the uniform dataset where it was $100 \%$ confident and had $10 \%$ support (480 instances). When evaluated on the million-instance set it was still $100 \%$ confident, and fully covered the entire class (47662 instances).

\subsubsection{Concept of one pair}

Another rule discovered while training on the uniform dataset made use of the same GenFunction described in the previous example.

GF: $\$ A$ NEV (class $=$ Rank $)=2\{$ Variable $\}]$

If $(\mathrm{C} 5 !=\mathrm{C} 2) \wedge(\$ \mathrm{~A}=1) \wedge(\mathrm{S} 4 !=4) \wedge(\mathrm{C} 1 !=\mathrm{C} 5) \wedge(\mathrm{C} 1 !=\mathrm{C} 2) \wedge(\mathrm{S} 4 !=\mathrm{S} 5)$ then $($ CLASS $=1)$

At first glance this rule appears to be complex and perhaps over-specialized. $($ Class $=1)$ indicates that the hand is classified as a simple pair, and the reason that the $(\mathbf{S A}=1)$ does 
not cover it alone is because some hands that belong to other classes contain a pair as a subset. Therefore standing unqualified, using $(\mathbf{S A}=\mathbf{1})$ to classify any particular hand as a pair is incorrect. When evaluated using the million-instance dataset it incorrectly classifies 1424 instances, which is exactly the number of full house hands in the test set. Although the confidence is still very good at $99.663 \%$, a more specialized rule could cover the class with $100 \%$ confidence.

Looking at what this rule does, term by term:

1) (C5 != C2); excludes a pair between card \#5 and card \#2.

2) ( $\mathbf{S A}=1)$; means that exactly one pair of equally ranks cards exists.

3) (S4 != 4); excludes suit \#4 (Ex: Card \#4 is not a club). This is a useless term.

4) (C1 != C5); excludes a pair between card \#1 and card \#5.

5) (C1 := C2); excludes a pair between card \#1 and card \#2.

6) (S4 != S5); means that the suits of cards \#4 and \#5 are different.

After examining the terms we know that we have a pair, and Card \#1, Card \#2, and Card \#5 represent three different values. Knowing that there are only five cards in the hand, this excludes four of a kind. It also means that if one pair exists, then it has to be a match between: $(1,3),(1,4),(2,3),(2,4),(3,4),(3,5)$.

In order to construct a hand containing three of a kind it means that cards \#3 and \#4 must be the same as each other, and also the same as card \#1, card \#2, or card \#5. The problem with this is that the constraint of $(\mathbf{S A}=\mathbf{1})$ can no longer be satisfied involving either card 
$\# 3$ or card \#4. To clarify, if both cards \#3 and \#4 are the same and help to make a three of a kind using one of the other cards (Ex: $\mathbf{3}, 2, \mathbf{3}, \mathbf{3}, 5)$, then an exclusive pair does not exist. Because all of the combinations that satisfy $(\$ \mathbf{A}=\mathbf{1})$ involve one or both of these cards, it follows that hands containing three of a kind do not qualify. This also means that full house hands are excluded, as this class requires three of a kind as a subset.

All straight hands, as well as the subset classes (straight flush and royal flush) are excluded because more than one equal rank necessarily interrupts any straight. Also excluded is the flush, which also has the subset classes of straight flush and royal flush, because more than one card of equal rank cannot have the same suit.

The $f$ lush hands are also excluded by the (S4 $!=\mathbf{S 5}$ ), although this term is redundant for reasons already stated.

\section{Class summary}

Royal Flush: Excluded by $(\$ A=1)$

Straight Flush: Excluded by $(\$ A=1)$

Four of a kind: Excluded by $(\$ A=1)$

Full house: Excluded by $(\$ A=1) \wedge(\mathrm{C} 1 !=\mathrm{C} 5) \wedge(\mathrm{C} 1 !=\mathrm{C} 2) \wedge(\mathrm{C} 5 !=\mathrm{C} 2)$

Flush: Excluded by $(\$ A=1)$

Straight: Excluded by $(\$ A=1)$

Three of a kind: Excluded by $(\$ A=1) \wedge(\mathrm{C} 1 !=\mathrm{C} 5) \wedge(\mathrm{C} 1 !=\mathrm{C} 2) \wedge(\mathrm{C} 5 !=\mathrm{C} 2)$

Two pairs: Excluded by $(\$ A=1)$ 
One pair: Allowed by $(\$ A=1)$

Nothing: Excluded by $(\$ A=1)$

The only type of hand that is not excluded is one pair, and while this rule does not cover the entire class of one pair poker hands it is $100 \%$ correct for all of the instances that it matches.

\subsubsection{Second example of one pair}

In another experiment a second system for classifying one pair was discovered, and it is interesting because it is less intuitive to people who actually know the rules for poker. It contains the following two Gen-Functions and single accompanying rule:

$$
\begin{aligned}
& \text { GF: } \$ A[N E V(\text { class }=\text { Rank })=2\{\text { Variable }\}] \\
& \text { GF: } \$ E[N E V(\text { class }=\text { Suit })=3\{\text { Variable }\}] \\
& \text { If }(\$ A=\$ E) \wedge(\$ A=1) \text { then }(\text { CLASS }=1)
\end{aligned}
$$

This translates to:

1) There is exactly one pair of cards with equal rank. (Ex: Two Kings).

2) There are exactly three cards of the same suit. (Ex: Three hearts).

3) This hand is a pair.

At first glance it might not be obvious that this is $100 \%$ confident, but excluding each class shows that the rule is correct. As in the previous example, constraint (1) excludes 
the various flush and straight hands, as well as two pairs and nothing. The flush, straight flush, and royal flush are redundantly excluded by (2) because exactly three out of the five cards have the same suit. The only classes missing are the three of a kind, and full house.

For the three of a kind hand, consider the following example: $(6,6, X, Y, Z)$, where the three last variables are available for any rank.

In order to have three matching ranks it would require that either:

1) $\mathrm{X}, \mathrm{Y}$, or $\mathrm{Z}$ need to be the value 6 . The problem with this is that (1) will no longer be valid because the pair is transformed into three of a kind.

2) $X, Y$, and $Z$ all have to be given the same value, not equal to 6 . This will mean that there is a pair made up from the first two cards, as well as a three of a kind using the last three cards. This would now be classified as a full house, which makes three of a kind impossible to create given the constraints.

At this point it might appear that the full house is also a valid hand; consider the previous example with some additional suit information: $\left(6 \mathrm{H}, 6 \mathrm{D}, 8_{-}, 8_{-}, 8_{-}\right)$. The suits for the first two cards are hearts and diamonds respectively, while the last three cards are unspecified and can be labeled arbitrarily. The problem is that (2) specifies that exactly three cards must have the same suit, which means that at least two of the last three cards must have the same suit. Given that two equally ranked cards cannot have the same suit in a standard deck of cards, this constraint makes it impossible to create a full house. 
Therefore, through the process of class exclusion based on constraints, any five cards following these two simple rules must classify as a pair. This is not something that is intuitive to human players because the suit of a card is rarely considered unless they are all the same. However, to a learning algorithm that is unaware of the higher level concepts, these rules constitute a simple way of classifying a relatively large percentage of this domain.

\subsubsection{Concept of a flush}

Also during the same experiment while training on the uniform dataset, EvRFind discovered a series of rules that partially covered the set of flush hands.

GF: $\$$ T $[$ NEV (class $=$ Suit $)=5\{$ Variable $\}]$

$\mathrm{MV}: \# \mathrm{~N}[(\mathrm{C} 1>\mathrm{C} 2) \wedge(\mathrm{S} 5=\mathrm{S} 2)]$

MV: \#AU $[(\# N) \wedge(\$ T=1)]$

$[\mathrm{C} 100.00 \%, \mathrm{~S} 0.83 \%, \mathrm{R}=40, \mathrm{~W}=0]:$ If $(\mathrm{C} 2=4) \wedge(\# \mathrm{AU})$ then $(\mathrm{CLASS}=5)$

$[\mathrm{C} 100.00 \%, \mathrm{~S} 0.73 \%, \mathrm{R}=35, \mathrm{~W}=0]:$ If $(\mathrm{C} 2=2) \wedge(\# \mathrm{AU})$ then $(\mathrm{CLASS}=5)$

$[\mathrm{C} 100.00 \%, \mathrm{~S} 0.67 \%, \mathrm{R}=32, \mathrm{~W}=0]:$ If $(\mathrm{C} 2=6) \wedge(\# \mathrm{AU})$ then $(\mathrm{CLASS}=5)$

$[\mathrm{C} 100.00 \%, \mathrm{~S} 0.60 \%, \mathrm{R}=29, \mathrm{~W}=0]:$ If $(\mathrm{C} 2=3) \wedge(\# \mathrm{AU})$ then $(\mathrm{CLASS}=5)$

$[\mathrm{C} 100.00 \%, \mathrm{~S} 0.52 \%, \mathrm{R}=25, \mathrm{~W}=0]:$ If $(\mathrm{C} 2=5) \wedge(\# \mathrm{AU})$ then $(\mathrm{CLASS}=5)$

$[\mathrm{C} 100.00 \%, \mathrm{~S} 0.25 \%, \mathrm{R}=12, \mathrm{~W}=0]:$ If $(\mathrm{C} 2=9) \wedge(\# \mathrm{AU})$ then $(\mathrm{CLASS}=5)$

$[\mathrm{C} 100.00 \%, \mathrm{~S} 0.21 \%, \mathrm{R}=10, \mathrm{~W}=0]:$ If $(\mathrm{C} 2=8) \wedge(\# \mathrm{AU})$ then $(\mathrm{CLASS}=5)$ 
These seven rules cover about $38 \%$ of class \#5. They appear to have started as one rule and the remaining six were discovered by Hill-Climbing. The \#AU Meta-Variable makes use of another Meta-Variable and a Gen-Function. The Gen-Function guarantees that the suits of all five cards are the same, which limits it to some kind of flush.

The other Meta-Variable, \#N makes sure that $(\mathbf{C 1}>\mathbf{C 2})$. By itself, in the general case, this doesn't help when trying to determine the type of flush. When a value is specified for $\mathrm{C} 2$, however, it would help with excluding a royal flush because the specified rank is outside of the range $\{10,11,12,13,1\}$.

What is missing from these rules is the recognition of a straight flush. This hand could be misclassified because it is not specifically excluded. The most likely cause for this is a lack of counter examples in the data. The number of instances of straight flush hands is so few that no counter examples were referenced while evolving these rules.

One important thing to notice is that a Meta-Variable was created from basic components and eventually used to build higher level rules. If EvRFind were to keep searching at this point then the task is potentially less difficult because it can use what has already been discovered.

\subsubsection{Concept of a full house}

Intuitively the full house seems like it might be difficult to classify, but it was discovered very early in another experiment that used the uniform dataset for training. 
GF: $\$ A$ NEV(class $=$ Rank $)=3\{$ Variable $\}]$

GF: \$D $[$ NEV $($ class $=$ Rank $)=2\{$ Generalized $\}]$

If $(\$ A=1) \wedge(\$ D=\$ A)$ then $($ CLASS $=6)$

This translates to: "If there is exactly one three of a kind within the five card ranks, and the number of pairs is also equal to this number, then the hand is a full house". This is essentially the definition. No matter how large the testing set, it is obvious to a human observer that this rule is $100 \%$ confident and will cover all of the instances in the class.

\subsubsection{Concept of four of a kind}

In older versions of EvRFind, before Gen-Functions were implemented, this is one of the classes that routinely achieved $100 \%$ confidence and covered every instance of this class in the dataset. The older rules were similar to the following:

$$
\begin{aligned}
& \text { If }(\mathrm{C} 1=\mathrm{C} 2) \wedge(\mathrm{C} 2=\mathrm{C} 3) \wedge(\mathrm{C} 3=\mathrm{C} 4) \text { then }(\mathrm{CLASS}=7) \\
& \text { If }(\mathrm{C} 1=\mathrm{C} 2) \wedge(\mathrm{C} 2=\mathrm{C} 3) \wedge(\mathrm{C} 3=\mathrm{C} 5) \text { then }(\mathrm{CLASS}=7) \\
& \text { If }(\mathrm{C} 1=\mathrm{C} 2) \wedge(\mathrm{C} 2=\mathrm{C} 4) \wedge(\mathrm{C} 4=\mathrm{C} 5) \text { then }(\mathrm{CLASS}=7) \\
& \text { If }(\mathrm{C} 1=\mathrm{C} 3) \wedge(\mathrm{C} 3=\mathrm{C} 4) \wedge(\mathrm{C} 4=\mathrm{C} 5) \text { then }(\mathrm{CLASS}=7) \\
& \text { If }(\mathrm{C} 2=\mathrm{C} 3) \wedge(\mathrm{C} 3=\mathrm{C} 4) \wedge(\mathrm{C} 4=\mathrm{C} 5) \text { then }(\mathrm{CLASS}=7)
\end{aligned}
$$


To cover the class always takes at least five rules, and because of the possible variance it often took many generations to evolve them all. The introduction of Gen-Functions not only compressed the number of rules, but the knowledge was discovered much faster. The Gen-Function and corresponding rule for achieving $100 \%$ confidence for every instance in the class are:

GF: $\$ L$ NEV(class $=$ Rank $)=4\{$ Generalized $\}]$ If $(\$ L=1)$ then $($ CLASS $=7)$

Translation: "If there are exactly four cards with the same rank then the hand is a four of a kind". As with the case of full house, this is basically the definition that is understood by human players, so it is easy to recognize that it will hold for any valid hand.

\subsubsection{Concept of three of a kind}

It is trivial for EvRFind to discover hands that contain three of a kind, but $100 \%$ confidence would require that the full house hands be excluded. While training during different experiments on the uniform dataset, EvRFind discovered two suitable rules.

GF: $\$ A$ NEV $($ class $=$ Rank $)=2\{$ Generalized $\}]$

GF: \$B [NEV(class = Rank) $=3\{$ Generalized $\}]$

GF: $\$ C[N E V($ class $=$ Rank $)=4\{$ Generalized $\}]$

1: If $(\$ B>\$ A)$ then $($ CLASS $=3)$ 
2: If $(\$ A=\$ C) \wedge(\$ B=1)$ then $($ CLASS $=3)$

Either of these rules will independently answer every instance of the class with $100 \%$ confidence. Rule \#1 reads as: "If the number of three of a kinds is greater than the number of pairs, then the hand is a three of a kind'. Because there can exist, at most, one three of a kind, and the comparison operator is strictly greater than, it means that there must be one three of a kind and no pairs. This successfully excludes the full house.

Rule \#2 is less intuitive because it relies on a trick that EvRFind discovered to make rules more parsimonious. The term $(\mathbf{S A}=\mathbf{S C})$ means that the number of pairs is equal to the number of four of a kinds. It doesn't require much analysis to realize that there is only one value for this: zero. The reason is that you cannot have a pair and a four of a kind in the same hand given that there are only five cards. The semantically equivalent set of terms that a human might use is: $(\mathbf{\$ A}=\mathbf{0})$ and $(\mathbf{} \mathbf{C}=\mathbf{0})$.

When combining the facts that are no pairs but there is a three of a kind, it follows that the full house is excluded, and the hand can only be a three of a kind.

\subsubsection{Concept of nothing}

In many systems the learning algorithm will make assumptions when the class cannot be determined. Typically the assumed class is the majority class, and the results can be very good in cases where the learning algorithm works well at identifying non-majority classes before assuming the default. As was seen earlier in section 6.6, Classifying the Poker 
Hand Dataset, starting on page 187, there were experiments performed using several algorithms available in Weka. After reviewing the results it was obvious that using the majority is not always the best choice. Especially in cases where the training set is not representative of the actual data is it important to try other techniques before making assumptions based on statistics.

For this set of experiments, EvRFind did not make any assumptions about class, and therefore attempted to build rules to specifically classify all instances. This experiment was performed by training on the true distribution dataset, and it later achieved $99.76 \%$ accuracy on the test set with 997606 correctly classified instances. There were no missed instances, and the majority of errors (2382) were made by the top rule in the hierarchy. The top rule required several Gen-Functions to work and was not perfect, but is very much along the way to successfully classifying nothing.

$$
\begin{aligned}
& \text { GF: \$A }[N E V(\text { class }=\text { Rank })=3\{\text { Variable }\}] \\
& \text { GF: } \$ D[N E V(\text { class }=\text { Rank })=2\{\text { Generalized }\}] \\
& \text { GF: } \$ \text { J }[N E V(\text { class }=\text { Rank })=4\{\text { Generalized }\}] \\
& \text { GF: } \$ N[\text { RGC (class = Rank })]
\end{aligned}
$$$$
\text { If }(\$ N !=5) \wedge(\$ A=\$ J) \wedge(\$ D=\$ A) \text { then }(\text { CLASS }=0)
$$

This rule breaks down into the following components: 
1) RGC(class = Rank); This function returns the range of ranks in the current hand. If all ranks were the same then the range would be equal to one, although this could not occur in the poker hand dataset because five different cards cannot have the same rank.

2) (\$N != 5); The span of card ranks is not exactly five, which means that there cannot be five cards in a row, except for the case of a straight with the Ace in the high position $\{10$, Jack, Queen, King, Ace $\}$.

3) $(\mathbf{S A}=\mathbf{S J})$; The number of three of $a$ kinds is equal to the number of four of $a$ kinds. As described earlier this can only mean that they are both equal to zero, so this expands to: $(\mathbf{S A}=\mathbf{0})$ and $(\mathbf{S J}=\mathbf{0})$.

4) $(\mathbf{~} \mathbf{D}=\mathbf{S A})$; Similar to (3) this constraint ensures that there are equal numbers of pairs and three of a kinds. By itself this term could be used, at least in part, to classify a full house. In the context of this rule it has a different meaning: "There are no pairs". The reason for this is the combination of terms between (3) and (4); $(\mathbf{S A}=\mathbf{~ \$ D}=\mathbf{\$ J})$ means that there can be no doubles, no triples, and no quadruples in the card ranks.

5) (CLASS = 0); If all of these constraints are satisfied then the hand is classified as nothing.

Putting all this together it is known that the hand does not contain a pair, or a three of a kind, or a four of a kind, or a full house, or a non-ace-high straight. Although this rule was very good it missed the concept of flush. Extant in the set of Gen-Functions in the same hierarchy was: 
GF: $\$ P[N E V($ class $=$ Suit $)=5\{$ Generalized $\}]$

It is expected that given more time, EvRFind would have created a new rule that contained $(\$ \mathbf{P}=\mathbf{0})$ or $(\mathbf{\$ P} !=\mathbf{1})$. Had this occurred then it would have removed all regular flushes (1996 of them), as well as straight flushes (which were already removed because the Ace is necessarily low in all straight flush hands), and the royal flush hands ( 3 of them). It would have only missed the non-flush straights that have cards \{Ten, Jack, Queen, King, Ace $\}$ in any order.

\subsubsection{Concept of straight}

As stated in the initial description of the poker hand dataset, the straight hand is a very difficult concept for learning algorithms that use the standard set of comparison operators $\{=,<,>,<=,>=, \neq\}$ because there is no recognition of the ranks between two cards. The difficulty is compounded because of the two different positions that an Ace can occupy, making it possible to have both Ace-high and Ace-low straights.

EvRFind is capable of dealing with straights in several ways, and in the current revision the most generic rules make use of the RGC function. This function calculates the span between the maximum and minimum values, and can be useful when combined with other information. For example, if the RGC of ranks is five, and there are no equal values in the hand, then the hand is some kind of straight. Following are some rules that EvRFind discovered while trying to accurately classify the simple straight: 
GF: $\$ A$ [NEV $($ class $=$ Rank $)=2\{$ Generalized $\}]$

GF: $\$ C[N E V($ class $=$ Rank $)=3\{$ Generalized $\}]$

GF: $\$ H[N E V($ class $=$ Rank $)=4\{$ Variable $\}]$

GF: $\$ K$ [NEV(class $=$ Suit $)=5\{$ Generalized $\}]$

GF: \$N $[$ RGC (class $=$ Rank $)]$

$M V: \# S[(\$ H=\$ A) \wedge(\$ N=5)]$

If $(\$ A=\$ C) \wedge(\$ K=0) \wedge(\# S)$ then $($ CLASS $=4)$

This rule breaks down into the following components:

1) $(\mathbf{S A}=\mathbf{\$ C})$; The number of pairs and three of a kinds is equal. This is either going to be a full house, or neither of these will be present.

2) $(\mathbf{\$ K}=\$ \mathbf{0})$; It is not a flush because there are no cases where five cards have the same suit.

3) $(\# \mathbf{S})=(\$ H=\$ A) \wedge(\$ N=5)$

4) $(\mathbf{S H}=\mathbf{\$ A})$; The number of four of a kinds is equal to the number of pairs, which we know must be zero. This also means that because of the first term, $(\mathbf{S A}=\mathbf{\$ C})$, the number of three of a kinds is also zero.

5) $(\$ \mathbf{N}=5)$; This means that within the five cards, where we already know that there are no duplicate ranks, the span is five. 
This rule effectively covers all of the straights that do not include an Ace in the high position. The straight flush and royal flush subsets are excluded because of the second term.

The only remaining instances of straight hands that are not covered are combinations of $\{10$, Jack, Queen, King, Ace $\}$, in any order with at least two different suits. EvRFind has not yet discovered a general way to classify these, but has had some success in creating very low-support rules to cover small numbers of instances.

GF: \$L $[$ NEV(class $=$ Suit $)=4\{$ Generalized $\}]$

GF: $\$ O[M N C($ class $=$ Rank $)]$

GF: $\$ R[N E V($ class $=$ Suit $)=5\{$ Variable $\}]$

$\mathrm{MV}: \# \mathrm{X}[(\mathrm{C} 5>\mathrm{C} 2) \wedge(\$ \mathrm{O}=1)]$

If $(\$ R=\$ L) \wedge(C 2=10) \wedge(C 4>C 5) \wedge(S 2=2) \wedge(\# X)$ then $($ CLASS $=4)$

If $(\mathrm{S} 5=\mathrm{S} 4) \wedge(\mathrm{C} 4>10) \wedge(\mathrm{C} 4<\mathrm{C} 2) \wedge(\mathrm{C} 3>\mathrm{C} 2) \wedge(\$ \mathrm{O}=1)$ then $(\mathrm{CLASS}=4)$

Both of these rules are similar in that they specify the minimum rank to be one, which means that the hand contains an Ace. The other common feature is that the other card ranks have at least one ten, as well as some that are guaranteed to be higher than ten. 
Basically they eliminate hands from the training set by using very specific constraints, but they do not necessarily work for unseen data.

Covering the remaining straight hands in a general way is not obvious, but looking at the properties for doing so might help to inspire new Gen-Functions. Some of the properties of these missing hands are:

1) The range of card ranks is 13 .

2) The minimum rank is 1 (Ace) and the maximum is 12 (King).

3) The cards can be in any order.

4) The cards cannot all have matching suits.

5) There are no duplicate ranks.

These properties can all be expressed using the current grammar, but this is not rich enough to create a generalized function to cover the remaining instances. In considering what else might be needed it is important to see what cannot be easily expressed by the current set of Gen-Functions.

6) It is always the same set of card ranks: $\{1,10,11,12,13)$.

7) Exactly four of the cards are within one rank of each other.

8) The number of cards with ranks above 9 is four.

9) The number of cards with ranks below 10 is one.

10) The number of cards with rank equal to $\{1$ or 10 or 11 or 12 or 13$\}$ is five. 
The inclusion of Gen-Functions to cover some or all of these additional observations has the potential to give EvRFind expressions powerful enough to cover all of the remaining instances.

\subsubsection{Concept of straight flush}

The straight flush hand relies on successfully being able to classify both straight hands and flush hands, and then realizing that this is a related subset of both. Fortunately, the straight flush has a helpful property: Any straight flush containing an Ace will be an Acelow straight, because otherwise it would be a royal flush.

All of the required straights, as well as the concept of a flush, have been previously discovered by EvRFind. It is not a surprise that EvRFind discovered the following GenFunctions and accompanying rule.

GF: \$Q [RGC(class $=$ Rank $)]$

GF: \$R $[N E V($ class $=$ Suit $)=5\{$ Variable $\}]$

If $(\$ R=1) \wedge(\$ Q=5)$ then $($ CLASS $=8)$

This rule covers the entire class of straight flush with $100 \%$ accuracy. 


\subsubsection{Concept of royal flush - Initially}

The royal flush is difficult for the very reason that was previously mentioned concerning misclassification of the straight hand. Unlike the straight where only a small subset of the hands contains an Ace-high straight, all of the royal flush hands are Ace-high straights.

During the initial experiments with EvRFind it was able to classify several royal flush hands using very specific rules. However, the performance is very poor on unseen data. An example of one such rule is:

$$
\begin{aligned}
& \text { GF: } \$ \text { Q }[\text { RGC }(\text { class }=\text { Rank })] \\
& \text { GF: } \$ \text { R }[\text { NEV }(\text { class }=\text { Suit })=5\{\text { Variable }\}]
\end{aligned}
$$

$\mathrm{MV}: \# \mathrm{~W}[(\mathrm{~S} 4 !=3) \wedge(\$ \mathrm{R}=1)]$

If $(\mathrm{C} 3>9) \wedge(\$ Q=13) \wedge(C 2>6) \wedge(\# W)$ then $($ CLASS $=9)$

The most useful components of this rule are that it ensures the hand is a flush, and that it is not a straight flush. It cannot be a straight flush because there is no such instance within the domain that has a range of 13 for rank. The reason that it is $100 \%$ confident in the training set is because the other terms render this rule over-fit, which eliminates the potential counter examples from the training set. 
This case also highlights one of the problems with assuming classes. Consider an experiment where EvRFind is not able to distinguish Ace-high straights, and as the last rule it assumes that all unclassified instances should be classified as a straight. This would correctly classify most of the remaining instances. Unfortunately by following this strategy, all of the royal flush hands that are not addressed by over-fit rules will be misclassified.

\subsubsection{Concept of royal flush - Revisited}

With the success being realized through the use of Gen-Functions, an effort was made to include more primitive functions within the library. The most recent additions included mathematical primitives such as calculating the sum or product of values within an example instance. A complete list of Gen-Function primitives that are included in the current version can be seen in Chapter 5.11.3.7: List of Implemented Gen-Functions, starting on page 145 .

At the time that these new additions were implemented, it was not expected that they might have any effect on the ability of EvRFind to classify poker hands. Interestingly, a few of the new functions had a positive effect on how the royal flush hand was being classified. The following example is one solution that was found to make use of the "product" function.

\section{$\underline{\text { Solution using Product }}$}

GF: \$A [LFC(class = Suit)] "Least frequent suit in the hand" 


$$
\begin{aligned}
& \text { GF: \$B [MFC(class = Suit)] } \quad \text { "Most frequent suit in the hand" } \\
& \text { GF: } \$ M[M N C \text { (class = Rank)] } \\
& \text { GF: } \$ \text { O }[\text { PRC(class = Rank)] } \quad \text { "Product of ranks in the hand" }
\end{aligned}
$$

If $(\$ A=\$ B) \wedge(\$ O=17160) \wedge(\$ M=1)$ then $(C L A S S=9)$

This rule translates to: "If the least-common-suit is the same as the most-common-suit (which, in the case of 5 cards, means that all cards must have the same suit) and the product of the ranks is 17160 and the minimum rank in the set is 1 (Ace) then the hand is a royal flush".

The first term, $(\$ \mathrm{~A}=\$ \mathrm{~B})$, simply ensures that this hand is a flush. The second term states that the product of the ranks must be equal to 17160 , and this requires some analysis.

$17160=10 \times 11$ (Jack) $\times 12$ (Queen) $\times 13$ (King) $\times 1$ (Ace)

Knowing that the value 17160 is the product of these five ranks is useful, but only if this product is unique and can only be achieved with these cards. Unfortunately, there are two other combinations of ranks that have the same product.

$17160=2 \times 5 \times 11$ (Jack) $\times 12$ (Queen) $\times 13$ (King)

$17160=2 \times 6 \times 10 \times 11$ (Jack) $\times 13$ (King) 
Upon inspection of these two sets of card ranks it is easy to see that they have a couple of properties in common. The first is that the Ace (rank 1) and a second card were replaced by two other cards, and the second commonality is that a card of rank 2 is one of the replacement cards. It follows that if the rule can be specialized such that at least one of these two properties can be ruled out then the value of 17160 will guarantee the proper set of ranks. This is where the third term, $(\$ M=1)$, is important.

The third term guarantees that the minimum rank within the set of cards is a 1 , which means that there must be an Ace in the hand. With this guarantee, taking the first two terms into account, it means that the hand represents a royal flush. Even more significant is the fact that this simple rule is $100 \%$ accurate, and covers $100 \%$ of all royal flush hands.

Two interesting alternative solutions, shown below, were discovered in subsequent experiments. These were only found after the "average" and "sum" functions were implemented, and as in the case of the "product" function, it was not expected that either of these would contribute to the classification of poker hands.

\section{Solution using Sum}
GF: $\$ A$ [LFC(class $=$ Suit $)]$
"Least frequent suit in the hand"
GF: \$B [MFC(class $=$ Suit $)]$
"Most frequent suit in the hand"
GF: \$L [RGC(class $=$ Rank $)]$
"Range of the ranks in the hand"
GF: \$Q [SMC(class = Rank $)]$
"Sum of the ranks in the hand" 
If $(\$ A=\$ B) \wedge(\$ Q=47) \wedge(\$ L=13)$ then $(C L A S S=9)$

This rule translates to: "If the least-common-suit is the same as the most-common-suit (which, in the case of 5 cards, means that all cards must have the same suit) and the sum of the ranks is 47 and the range of the ranks is 13 then the hand is a royal flush".

\section{Solution using Average}

The solution using the "average" function is basically the same as the solution that uses the "product" function. The rule differs by checking to see if the "average" is 9.4, rather than verifying that the "product" is 17160 .

Using the average has the additional benefit of not having to check for the Ace. The reason for this is because the average is unique for the ranks that are required. Given that the suits are all the same, checking to ensure that the average is 9.4 is enough to correctly classify $100 \%$ of the royal flush hands.

\subsubsection{Improving accuracy through rule re-ordering}

The best reported accuracy for the experiments was $99.956 \%$, and it seems likely that EvRFind will be able to achieve better than this in the future. This suspicion comes from the number of corrections that EvRFind makes. A correction is counted as the successful classification of an instance using a rule in the hierarchy, but only in cases where the instance had been previously misclassified. 
When a correction is made by EvRFind then it does not contribute to the predictive accuracy, but it does indicate that within the hierarchy there exists a rule that classifies the instance correctly. With this knowledge it may be possible to modify the rules themselves through specialization, or possibly even the simpler solution of reordering the hierarchy such that the correct rule is applied first.

To test this theory another experiment was performed; the same set of rules was used on the same test set, but they were first sorted by training-confidence. This meant that any rule with $100 \%$ confidence on the training set, no matter how few instances it covered, was used before the top rule in the hierarchy.

The top rule was moved to the last position in the hierarchy, and although the final confidence did not reach $100 \%$, several instances that were being improperly classified were now being addressed by more accurate rules. As a result, the overall predictive accuracy rose from $99.956 \%$ to $99.99 \%$, where the sorted hierarchy correctly answers 999,914 out of $1,000,000$ instances. For this experiment, all of the misclassifications are in the straight class.

\subsection{Summary of EvRFind experiments}

During experiments EvRFind discovered several $100 \%$ confident, full coverage rules for the poker hand dataset. Each of these rules answers an entire class with $100 \%$ confidence, and does not misclassify any instances belonging to other classes. The classes for which 
these rules exist are: One pair, two pairs, three of a kind, full house, four of a kind, straight flush, and royal flush. Utilizing all of these rules would ensure that at least $49.29 \%$ of the entire domain is properly classified without any risk of misclassification.

If the cost of misclassification is acceptable then EvRFind is even more useful because it can accurately classify all instances of nothing, as well as the majority of straight hands. The margin of error in these rules comes from the fact that they classify too many instances, but it is still important to note that most or all of the target class is properly classified. Combined with the coverage for the perfect rules, EvRFind uses a small number of rules to achieve at least $99.76 \%$ accuracy across the entire domain. 


\subsection{Summary of Poker Hand experiments}

None of the learning algorithms that were evaluated was able to accurately cover $100 \%$ of the poker hand dataset.

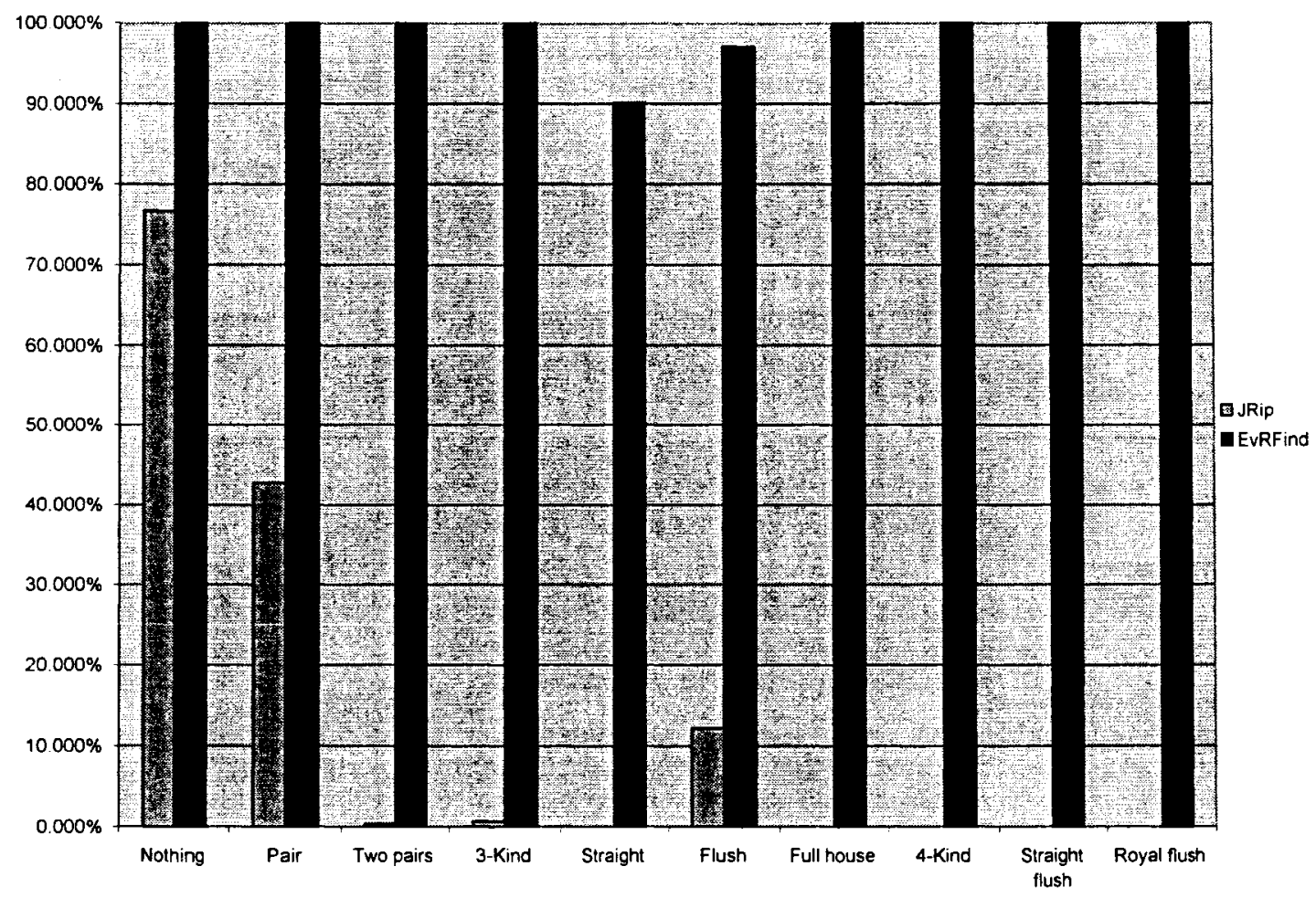

Figure 6.4: Class coverage comparison between EvRFind and JRip

This chart is a visual comparison of the class coverage achieved by EvRFind and the JRip algorithm on the poker hand dataset. The overall accuracy achieved by EvRFind is partly due to complete coverage of some classes, but also the partial coverage of other classes. Unlike JRip, the predictive models discovered by EvRFind do not prefer majority classes. 


\section{Chapter 7: Miscellaneous Dataset Experiments}

\subsection{Description}

When evaluating the performance of a learning algorithm it is important to compare results against those discovered by other methods. This can be done by performing experiments using other algorithms on the same data using the same parameters. Alternatively, duplicating other published experiments will highlight the differences between methods, and the features of an algorithm can be objectively determined. Without this type of analysis it is very difficult to assess the worth of any learning algorithm.

For the purpose of evaluating new and existing methods there are repositories of datasets available on the Internet. One such site is the UCI Machine Learning Repository [Blake, Murphy, 1998], which contains a number of datasets as well as results and analysis by various researchers.

Using previously published results has obvious advantages over re-creating experiments completely. The first is the time-saving benefit; it is much faster and easier to reference results as compared to rediscovering them. Secondly, it is often the case where the bestof-the-best results are reported.

The second point is true because the authors of published work are often very familiar with certain algorithms. In many cases these authors have written their own algorithms, 
and it is typically the case where the best-achieved results, using very fine-tuned parameters, are reported. Being unaware of the parameters used by a specific learning algorithm could inhibit another researcher from accurately re-creating these results. When previously published work is used then this is presumably not a concern, and each set of results is assumed to have the benefit of expert setup and testing.

In following this test plan, several datasets were downloaded from the UCI repository and examined by EvRFind. In most cases the configuration, such as the size of training and testing sets, were modeled after the previously published work. Details for each of these experiments are included in this chapter.

Several of the following experiments are duplicated in order to evaluate the hierarchyprotected-fitness option that was described in Chapter 5.5.6: Building the hierarchy, starting on page 92 , and previously evaluated on the poker hand dataset in Chapter 6.7.3: Evaluating the Hierarchy Protected Fitness option, starting on page 200.

The results discovered by EvRFind are presented in tabular format, and although some of the results highlight different features of the experiment, all of them use the following legend to describe the columns in the corresponding result table.

\section{Legend}

Pop size: The initial number of rules in the GA population.

TrSz: Size of the training set. 
TsSz: Size of the testing set.

Min \%: The minimum predictive accuracy (in 10 experiments).

Max \%: The maximum predictive accuracy.

AVG \%: The average predictive accuracy.

Missed: The average percentage of instances that were not covered by any rule.

Error \%: The average error rate.

Options:

HPF: Hierarchy protected fitness.

Non-HPF: Not using hierarchy protected fitness

MV: Using meta-variables

GF: Using gen-functions

\subsection{The Mushroom Dataset}

\subsubsection{Description}

The mushroom dataset contains 8124 records describing hypothetical samples corresponding to 23 species of gilled mushrooms in the Agaricus and Lepiota Family (drawn from [Lincoff, 1981]). There are 22 predictive attributes, and they are all nominally valued with varying numbers of possible values. There are missing values in the data, but these are restricted to a single attribute (\#11). 
The classification is binary: definitely edible or not. The negative class contains instances that are either poisonous or are of unknown edibility. The distribution is $4208(51.8 \%)$ definitely edible versus 3916 (48.2\%) not.

\subsubsection{Classification experiments and results}

Several published papers, such as [Kokai, Toth, Zvada, 2002], contain results of learning algorithms operating on the mushroom dataset. These results are duplicated in the following table:

\begin{tabular}{|l|c|c|}
\hline System & $\begin{array}{c}\text { Training } \\
\text { size }\end{array}$ & $\begin{array}{c}\text { Testing } \\
\text { error }\end{array}$ \\
\hline Stagger [Schlimmer, 1987] & 5416 & $5.0 \%$ \\
\hline Cart [Breiman, Friedman, Ohlsen, Stone, 1984] (2 fold CV) & 200 & $1.3 \%$ \\
\hline Cart & 300 & $0.9 \%$ \\
\hline PERLS [Cost, Salzberg, 1993] (10 fold CV) & 7311 & $0.3 \%$ \\
\hline Nearest neighbours [Cost, Salzberg, 1993] (10 fold CV) & 7311 & $0.0 \%$ \\
\hline 1R [Holte, 1993] & 8124 & $1.5 \%$ \\
\hline 1R & 5416 & $1.6 \%$ \\
\hline Neural Network [Yeung, 1991] & 100 & $5.9 \%$ \\
\hline JGA [Hekanaho, 1998] & 4000 & $0.0 \%$ \\
\hline REGAL [Neri, 1997] & 4000 & $0.0 \%$ \\
\hline REGAL & 1000 & $0.4 \%$ \\
\hline REGAL & 500 & $0.4 \%$ \\
\hline AQ [Michalski, Mozetic, Hong, Lavrac, 1986] & 4000 & $0.0 \%$ \\
\hline CN2 [Clark, Niblett, 1989] & 4000 & $0.0 \%$ \\
\hline
\end{tabular}

Table 7.1: Published results for the mushroom dataset

The systems listed in this table did well in classifying the mushroom dataset. Five of them were able to achieve $100 \%$ confidence, although only when training with almost half of the data. 
In [Duch et al, 1998] the authors described C-MLP2LN, which is a hybrid algorithm that uses a neural network for feature selection followed by a search-based optimization. In reference to the mushroom dataset the authors report that the following disjunctive rules for poisonous mushrooms have been discovered:

\begin{tabular}{|c|c|c|}
\hline \# & Terms in the antecedent & Missed \\
\hline 1 & (odor $\neq$ almond $) \wedge($ odor $\neq$ anise $) \wedge$ (odor $\neq$ none $)$ & 120 \\
\hline 2 & (spore print_color $=$ green $)$ & 48 \\
\hline 3 & (odor $=$ none $) \wedge($ stalk_surface_bel_ring $=$ scaly $) \wedge($ stalk_color_above ring $\neq$ brown $)$ & 8 \\
\hline 4 & (habitat $=$ leaves $) \wedge($ cap color $=$ white $)$ & 0 \\
\hline & Alternative rule & \\
\hline 4-a & $($ population $=$ clustered $) \wedge($ cap_color $=$ white $)$ & 0 \\
\hline
\end{tabular}

Table 7.2: Rules created by C-MLP2LN for the mushroom dataset

The above rules are $100 \%$ confident. The missed column indicates how many instances are missed after applying the current rule, as well as the previous rules in the list. After applying all four in the listed order, the poisonous mushroom instances are $100 \%$ classified. Implicitly, to achieve full coverage in the dataset there would have to be a fifth rule that classifies all remaining instances as edible.

\subsubsection{Evaluating C-MLP2LN rules in EvRFind}

To better compare the results of [Duch et all, 1998] against EvRFind, the four primary rules were converted and then evaluated against the full dataset.

$[\mathrm{C} 100.00 \%, \mathrm{R}=3796]:$ If $($ Odor $!=1) \wedge($ Odor $!=2) \wedge($ Odor $!=7)$ then $($ Edible $=2)$

$[\mathrm{C} 100.00 \%, \mathrm{R}=72]$ : If $(\mathrm{SP}-\mathrm{col}=5)$ then $($ Edible $=2)$

$[\mathrm{C} 100.00 \%, \mathrm{R}=40]:$ If $(\mathrm{Odor}=7) \wedge(\mathrm{SS}-\mathrm{b}-\mathrm{r}=2) \wedge(\mathrm{SC}-\mathrm{a}-\mathrm{r} !=1)$ then $($ Edible $=2)$ 
$[\mathrm{C} 100.00 \%, \mathrm{R}=8]:$ If $(\mathrm{Hab}=2) \wedge(\mathrm{C}-\mathrm{col}=9)$ then $($ Edible $=2)$

The accuracy reported by EvRFind for this set of rules is $48.20 \%$, or 3916 examples, which is $100 \%$ of the poisonous instances in the dataset.

\subsubsection{Results with EvRFind for the Mushroom dataset}

The dataset was examined with EvRFind using training set sizes of 500, 1000, 4000, and the full set of 8124 instances. For each training set the experiment was run ten times with different random number seeds.

\begin{tabular}{|c|c|c|c|c|}
\hline TrSz & TsSz & Min \% & Max \% & Avg \% \\
\hline 500 & 8124 & 98.42 & 99.90 & 99.56 \\
\hline 1000 & 8124 & 99.11 & 99.95 & 99.74 \\
\hline 4000 & 8124 & 99.41 & 99.95 & 99.79 \\
\hline 8124 & 8124 & 98.82 & 99.41 & 99.32 \\
\hline
\end{tabular}

Table 7.3: Results of EvRFind classifying the mushroom dataset

A more in-depth look at the results was warranted because EvRFind was not able to classify all of the instances with $100 \%$ accuracy. The confusion matrices for the experiments revealed that EvRFind was always able to classify instances that are definitely edible.

The best performance achieved by EvRFind was using eight rules to cover $100 \%$ of the dataset. The edible class was fully covered with four errors $(0.05 \%)$ misclassifying edible examples as poisonous, and all of poisonous instances were classified correctly.

$[\mathrm{R}=3796]$ : If $($ Odor $!=1) \wedge($ Odor $!=7) \wedge($ Odor $!=2)$ then $($ Edible $=2)$ 
$[\mathrm{R}=3920]:$ If $($ Odor $!=6) \wedge(\mathrm{SP}-\mathrm{col} !=5) \wedge(\mathrm{G}-\mathrm{size}=1) \wedge(\mathrm{SP}-\mathrm{col} !=4)$ then $($ Edible $=1)$

$[\mathrm{R}=96]:$ If $(\mathrm{Hab}=7) \wedge($ Bruise $!=2)$ then $($ Edible $=1)$

$[\mathrm{R}=72]:$ If $($ Stk-ro $=1) \wedge($ Stk-sh $!=2) \wedge($ SS-a-r $!=1) \wedge($ SP-col $!=8)$ then $($ Edible $=2)$

$[\mathrm{R}=192, \mathrm{~W}=4]$ : If $(\mathrm{Odor}=7) \wedge(\mathrm{SP}-\mathrm{col} !=5) \wedge(\mathrm{C}-$ surf $!=2) \wedge(\mathrm{SS}-\mathrm{b}-\mathrm{r} !=2)$ then $($ Edible $=1)$

$[R=32]:$ If $(\mathrm{SS}-\mathrm{a}-\mathrm{r}=3) \wedge(\mathrm{G}-\mathrm{spa}=1)$ then $($ Edible $=2)$

$[\mathrm{R}=8]:$ If $($ Stk-ro $!=1) \wedge(\mathrm{C}-\operatorname{col} !=3) \wedge($ SP-col $!=4) \wedge(\mathrm{C}-$ surf $!=1) \wedge($ pop ! $=6) \wedge(\mathrm{G}-$

size $=2)$ then $($ Edible $=2)$

$[\mathrm{R}=8]:$ If $(\mathrm{SP}-$ col $!=4) \wedge(\mathrm{C}-$ surf $!=1) \wedge(\mathrm{G}-$ size $=2) \wedge(\mathrm{C}-$ col $!=10) \wedge(\mathrm{SS}-\mathrm{a}-\mathrm{r} !=1)$ then

$($ Edible $=2)$

In the set of rules produced by EvRFind there were similarities with C-MLP2LN. The most obvious is that the first rule is semantically identical.

\begin{tabular}{|c|c|l|}
\hline$\#$ & System & Terms in the antecedent \\
\hline 3796 & C-MLP2LN & $($ Odor $!=1) \wedge($ Odor $!=2) \wedge($ Odor $!=7)$ \\
\hline 3796 & EvRFind & $($ Odor $!=1) \wedge($ Odor $!=7) \wedge($ Odor $!=2)$ \\
\hline 72 & C-MLP2LN & $($ SP-col $=5)$ \\
\hline 72 & EvRFind & $($ Stk-ro $=1) \wedge($ Stk-sh $!=2) \wedge($ SS-a-r $!=1) \wedge($ SP-col $!=8)$ \\
\hline 40 & C-MLP2LN & $($ Odor $=7) \wedge($ SS-b-r $=2) \wedge($ SC-a-r $!=1)$ \\
\hline 32 & EvRFind & $($ SS-a-r $=3) \wedge(G-s p a=1)$ \\
\hline 8 & EvRFind & $($ Stk-ro $!=1) \wedge($ C-col $!=3) \wedge($ SP-col $!=4) \wedge($ C-surf $!=1) \wedge($ pop $!=6) \wedge($ G-size $=2)$ \\
\hline 8 & C-MLP2LN & $($ Hab $=2) \wedge($ C-col $=9)$ \\
\hline 8 & EvRFind & $($ SP-col $!=4) \wedge($ C-surf $!=1) \wedge($ G-size $=2) \wedge($ C-col $!=10) \wedge($ SS-a-r $!=1)$ \\
\hline
\end{tabular}

Table 7.4: Comparison of poisonous rules in EvRFind and C-MLP2LN

The table above shows that although only the first rule is semantically equivalent, the overall result of fully classifying poisonous instances with $100 \%$ accuracy is attained using EvRFind. 
During the experiments there were other identical or semantically equivalent rules discovered by EvRFind, but these were not always present in the final results. One such example is:

$[\mathrm{C} 100.00 \%, \mathrm{R}=72]:$ If $(\mathrm{SP}-\mathrm{col}=5)$ then $($ Edible $=2)$

Instead of:

$[\mathrm{R}=72, \mathrm{~W}=0]:$ If $($ Stk-ro $=1) \wedge($ Stk-sh $!=2) \wedge($ SS-a-r $!=1) \wedge($ SP-col $!=8)$ then $($ Edible $=2)$

This first rule is more parsimonious and easier to understand but was not always discovered. An example-by-example check to see if the same instances are being covered was not done. However, when the second rule was substituted for the first in the same hierarchy the results were identical.

The only important difference between the two sets of rules is the last one in the hierarchy produced by EvRFind:

$[\mathrm{R}=8, \mathrm{~W}=0]:$ If $(\mathrm{SP}-$ col $!=4) \wedge(\mathrm{C}-$ surf $!=1) \wedge(\mathrm{G}-$ size $=2) \wedge(\mathrm{C}-$ col $!=10) \wedge(\mathrm{SS}-\mathrm{a}-\mathrm{r} !=$ 1) then (Edible $=2)$

This is the only rule out of the five that is not $100 \%$ confident when taken in isolation. This rule correctly classifies 8 instances and misclassifies 36 , which translates to a global 
confidence $18.18 \%$. When applied to the instances remaining after the four previous rules are used, however, the hierarchy confidence is $100 \%$ correct.

EvRFind made no use of the high-level Meta-Variables or Gen-Functions during rule discovery on the mushroom dataset.

\subsection{The Monk's Problems}

\subsubsection{Description}

The Monks problems [Thrun et al., 1991] are comprised of three training and three testing datasets. Each testing set contains 432 instances, and select subsets of these are used for training. The domains for all three datasets are the same and defined as:

\begin{tabular}{|l|l|}
\hline Attribute & Type / Information \\
\hline Class & Binary $\{0,1\}$ \\
\hline A1 & Nominal $\{1,2,3\}$ \\
\hline A2 & Nominal $\{1,2,3\}$ \\
\hline A3 & Nominal $\{1,2\}$ \\
\hline A4 & Nominal $\{1,2,3\}$ \\
\hline A5 & Nominal $\{1,2,3,4\}$ \\
\hline A6 & Nominal $\{1,2\}$ \\
\hline ID & Text; a unique symbol for each instance \\
\hline
\end{tabular}

Table 7.5: Attribute description for the Monks problems

The maximum size of the search space is: $3 * 3 * 2 * 3 * 4 * 2=432$, which means that the entire space is represented within each testing set.

Each of the problems is defined by a different target concept. The following functions determine which elements from the respective problem are classified as ' 1 ': 
Problem 1: $(\mathrm{A} 1=\mathrm{A} 2)$ or $(\mathrm{A} 5=1)$

Problem 2: Exactly two of $\{\mathrm{A} 1=1, \mathrm{~A} 2=1, \mathrm{~A} 3=1, \mathrm{~A} 4=1, \mathrm{~A} 5=1, \mathrm{~A} 6=1\}$

Problem 3: $[(\mathrm{A} 5=3)$ and $(\mathrm{A} 4=1)]$ or $[(\mathrm{A} 5 \neq 4)$ and $(\mathrm{A} 2 \neq 3)]$

The first two problems do not contain noise. The training set for the third problem contains $5 \%$ class noise. None of the datasets contain missing values.

\subsubsection{Classification experiments and results}

Several papers comparing the Monks problems have been published. The following table is a combination of results found in [Michalski, Mozetic, Hong, Lavrac, 1986], [Thrun et al., 1991], [Duch, Adamczak, Grabczewski, 2000], [Duch, Grudzinski, 1999] and is reproduced in part at <http://www.fizyka.umk.pl/kmk/projects/rules.html>.

\begin{tabular}{|l|c|c|c|l|}
\hline $\begin{array}{l}\text { Algorithm / } \\
\text { Method }\end{array}$ & Monk-1 & Monk-2 & Monk-3 & Source / Reference \\
\hline AQ17-DCI & 100.0 & 100.0 & 94.2 & Michalski et al., 1986 \\
\hline AQ17-HCI & 100.0 & 93.1 & 100.0 & Michalski et al., 1986 \\
\hline AQ17-GA & 100.0 & 86.8 & 100.0 & Michalski et al., 1986 \\
\hline Assistant Pro. & 100.0 & 81.5 & 100.0 & Thrun et al., 1991 \\
\hline mFOIL & 100.0 & 69.2 & 100.0 & Thrun et al., 1991 \\
\hline ID5R & 79.7 & 69.2 & 95.2 & Thrun et al., 1991 \\
\hline IDL & 97.2 & 66.2 & -- & Thrun et al., 1991 \\
\hline ID5R-hat & 90.3 & 65.7 & -- & Thrun et al., 1991 \\
\hline TDIDT & 75.7 & 66.7 & -- & Thrun et al., 1991 \\
\hline ID3 & 98.6 & 67.9 & 94.4 & Thrun et al., 1991 \\
\hline AQR & 95.9 & 79.7 & 87.0 & Thrun et al., 1991 \\
\hline CLASSWEB 0.10 & 71.8 & 64.8 & 80.8 & Thrun et al., 1991 \\
\hline CLASSWEB 0.15 & 65.7 & 61.6 & 85.4 & Thrun et al., 1991 \\
\hline CLASSWEB 0.20 & 63.0 & 57.2 & 75.2 & Thrun et al., 1991 \\
\hline PRISM & 86.3 & 72.7 & 90.3 & Thrun et al., 1991 \\
\hline ECOWEB & 82.7 & 71.3 & 68.0 & Thrun et al., 1991 \\
\hline MLP & 100.0 & 100 & 93.1 & Thrun et al., 1991 \\
\hline
\end{tabular}




\begin{tabular}{|l|c|c|c|l|}
\hline MLP+reg. & 100.0 & 100 & 97.2 & Thrun et al., 1991 \\
\hline Cascade correlation & 100.0 & 100 & 97.2 & Thrun et al., 1991 \\
\hline FSM, Gaussians & 94.5 & 79.3 & 95.5 & Duch et al, 1998 \\
\hline SSV & 100.0 & 80.6 & 97.2 & Duch et al, 1998 \\
\hline C-MLP2LN & 100.0 & 100.0 & 100.0 & Duch et al, 1998 \\
\hline kNN & 100.0 & 84.5 & 94.2 & Duch, Grudzinski, 1999 \\
\hline
\end{tabular}

Table 7.6: Published results for the Monks problems

The best results are using C-MLP2LN, which achieves $100 \%$ accuracy in all three problems. No other learning algorithm was able to achieve this. C-MLP2LN presents results for each problem in terms of rules and exceptions. Exceptions are instances in the data that are not properly classified and used in place of the more general rule.

The details of the rules discovered by C-MLP2LN are:

Monks-1: 4 rules, 2 exceptions

Monks-2: 16 rules, 8 exceptions

Monks-3: 3 rules, 4 exceptions

\subsubsection{Results using EvRFind for Monks-1}

For each of the Monk's problems, ten runs of EvRFind were executed using different random number seeds. All of the runs used the same parameter settings, which included searching for Meta-Variables and Gen-Functions.

The results for the Monks-1 problem were consistent across all experiments in EvRFind. The problem was always solved with $100 \%$ accuracy, and although the rules varied between experiments they were all similar to the following example: 


\section{Example rules discovered by EvRFind for solving Monks-1}

[A100.00\%]: If $(\mathrm{a} 2=\mathrm{a} 1)$ then (class $=1)$

$[\mathrm{A} 100.00 \%]:$ If $(\mathrm{a} 1 !=\mathrm{a} 2) \wedge(\mathrm{a} 6 !=\mathrm{a} 5) \wedge(\mathrm{a} 6=1)$ then $($ class $=0)$

[A72.41\%]: If $(\mathrm{a} 5=1)$ then $($ class $=1)$

$[\mathrm{A} 65.00 \%]:$ If $(\mathrm{a} 5=2) \wedge(\mathrm{a} 1 !=\mathrm{a} 2)$ then $($ class $=0)$

[A56.52\%]: If $(\mathrm{a} 5=4) \wedge(\mathrm{a} 1 !=\mathrm{a} 2)$ then $($ class $=0)$

[A47.37\%]: If $(\mathrm{a} 5=3) \wedge(\mathrm{a} 1 !=\mathrm{a} 2)$ then $($ class $=0)$

For each of these rules the value inside square brackets indicates the percentage of instances that were classified for the first time by the corresponding rule. This can provide some insight about redundancy in the dataset. For example, the third rule in the list is the second disjunctive component required to classify instances as class 1. Although it is correct $100 \%$ of the time, it was only used $72.41 \%$ of the time because the remainder was answered previously by rule $\# 1$. This suggests that $27.59 \%$ of the instances satisfy both of the target concepts required for inclusion in class 1 .

If this experiment were allowed to assume classes (such as class 0 ) then it would have been finished after discovering only rules \#1 and \#3. Combining these two rules using disjunction would yield the actual definition used to create the dataset. 


\subsubsection{Results using EvRFind for Monks-2}

The results for Monks-2 were more varied than Monks-1, although they were still quite good on average. Six out of the ten runs resulted in rule sets that achieved $100 \%$ accuracy on the testing set, while the average over all ten runs was $99.03 \%$.

EvRFind rules for solving Monks-2: Example \#1

GF: $\$ Q$ [NEV(class $=$ General ANT $)=2\{$ Constant $=1\}]$

If $(\$ Q=0)$ then (class $=0)$

If $(\$ Q=1)$ then $($ class $=1)$

For brevity the unused Gen-Functions and Meta-Variables were omitted from the above list. The two rules shown are the only two that were used, and achieved $100 \%$ confidence when answering all of the instances in the dataset.

Along with other solutions found during the experiments, the above solution was discovered independently several times. In some cases the predictive model was not able to achieve $100 \%$ accuracy, but often contained a small number of interesting rules.

EvRFind rules for solving Monks-2: Example \#2

GF: $\$ A$ NEV $($ class $=$ General ANT $)=2\{$ Generalized $\}]$

GF: $\$ F[N E V($ class $=$ General ANT $)=3\{$ Constant $=1\}]$ 
$[\mathrm{C} 100.00 \%, \mathrm{~S} 37.87 \%, \mathrm{R}=64]:$ If $(\$ \mathrm{~F}=\$ \mathrm{~A})$ then $($ class $=0)$

$[\mathrm{C} 100.00 \%, \mathrm{~S} 13.61 \%, \mathrm{R}=23]:$ If $(\$ \mathrm{~A}=3)$ then (class $=1)$

The first rule translates to: "If the number of times that exactly three ones are present is equal to the number of times that an unspecified pair is present, then the class is $0 "$. Because \$F specifies that there must be exactly three $1 \mathrm{~s}$, there cannot be exactly two of them (ex: $[1,1,1,2,2,3])$. If there are not three ones then because $(\$ F=\$ A)$ it means that there can be no pairs of values. If there can be no pairs, then there cannot be a pair of 1s. Given these constraints it is not possible to have exactly two $1 \mathrm{~s}$, and therefore the class is 0 .

The second rule translates to: "If there are exactly three distinct pairs of values then the class is 1". Although unintuitive, this rule makes sense when the details of the domain are understood. In particular, three of the attributes have three possible values $\{1,2,3\}$, two of the values have possible values of $\{1,2\}$, and only one of the attributes has four possible values $\{1,2,3,4\}$.

Considering that only one attribute can have value "4" then a pair of these is not possible. For there to be three distinct pairs then they must be two $1 \mathrm{~s}$, two $2 \mathrm{~s}$, and two $3 \mathrm{~s}$ in any allowed order (ex: $[3,3,2,1,1,2])$. When this is true then the "exactly two $1 \mathrm{~s}$ " rule is satisfied and the instance should be classified as 1 . It is $100 \%$ confident but does not cover the entire class because many instances exist where there are exactly two 1s 
without matching pairs of $2 \mathrm{~s}$ and $3 \mathrm{~s}$. Trivially, this rule will miss any instance that contains a pair of $1 \mathrm{~s}$ but also contains the value " 4 ".

\subsubsection{Results using EvRFind for Monks-3}

The results for Monks-3 were the worst for EvRFind and in no cases was 100\% accuracy achieved. As will be seen in the analysis to follow, this is the first indication that EvRFind does not handle class noise as well as some other learning algorithms. In the ten experiments the average accuracy was $97.01 \%$, with the best accuracy achieved being $98.61 \%$

EvRFind rules for solving Monks-3: Example \#1

GF $: \$ C[N E V($ class $=$ General ANT $)=2\{$ Variable $\}]$

$[\mathrm{C} 97.73 \%]:$ If $(\mathrm{a} 5 !=3) \wedge(\mathrm{a} 2 !=3) \wedge(\mathrm{a} 5 !=4)$ then $($ class $=1)$

$[\mathrm{C} 100.00 \%]:$ If $(\mathrm{a} 3 !=\mathrm{a} 4) \wedge(\mathrm{a} 2=3)$ then $($ class $=0)$

[C96.77\%]: If $(\mathrm{a} 5=4)$ then (class $=0)$

[C91.94\%]: If $(\mathrm{a} 2 !=3) \wedge(\mathrm{a} 5 !=4)$ then $($ class $=1)$

$[\mathrm{C} 100.00 \%]:$ If $(\mathrm{a} 4 !=1) \wedge(\mathrm{a} 2=3)$ then $($ class $=0)$

$[\mathrm{C} 100.00 \%]:$ If $(\mathrm{a} 3 \mathrm{~m} !=\mathrm{a} 4) \wedge(\mathrm{a} 3 !=\mathrm{a} 2) \wedge(\mathrm{a} 5 !=\mathrm{a} 3) \wedge(\mathrm{a} 5 !=\mathrm{a} 1) \wedge(\mathrm{a} 2 !=1) \wedge(\mathrm{a} 1 !=\mathrm{a} 3)$

then $($ class $=0)$

$[C 100.00 \%]:$ If $(a 5 !=a 4) \wedge(a 4 !=a 1) \wedge(a 1 !=a 3) \wedge(a 4 !=2) \wedge(a 4 !=a 2) \wedge(a 2 !=a 6) \wedge$ $(\$ C=1)$ then $($ class $=1)$ 
$[\mathrm{C} 100.00 \%]:$ If $(\mathrm{a} 5 !=\mathrm{a} 4) \wedge(\mathrm{a} 4 !=\mathrm{a} 1) \wedge(\mathrm{a} 5 !=4) \wedge(\mathrm{a} 1 !=\mathrm{a} 3) \wedge(\mathrm{a} 4 !=2)$ then $($ class $=1)$

$[\mathrm{C} 100.00 \%]:$ If $(\mathrm{a} 2 !=\mathrm{a} 6) \wedge(\$ \mathrm{~A}=1) \wedge(\mathrm{a} 4=\mathrm{a} 2)$ then $($ class $=0)$

$[\mathrm{C} 100.00 \%]:$ If $(\mathrm{a} 3 !=\mathrm{a} 2) \wedge(\mathrm{a} 4=\mathrm{a} 1) \wedge(\mathrm{a} 2 !=\mathrm{a} 6) \wedge(\$ C=1)$ then $($ class $=0)$

[C96.77\%]: If $(\mathrm{a} 2=3) \wedge(\mathrm{a} 5 !=\mathrm{a} 2)$ then $($ class $=0)$

$[C 77.27 \%]:$ If $(\mathrm{a} 5 !=\mathrm{a} 4) \wedge(\mathrm{a} 5 !=\mathrm{a} 3) \wedge(\mathrm{a} 2 !=\mathrm{a} 5) \wedge(\mathrm{a} 5 !=\mathrm{a} 6) \wedge(\mathrm{a} 1 !=\mathrm{a} 5)$ then $($ class $=0)$

The hierarchy shown above has a predictive accuracy of $97.22 \%$ on the testing set. Two subsets of these rules can be immediately recognized; the first is shown below, and contains all of the rules that classify the data as 1 . The second subset contains rules that are not $100 \%$ confident.

Subset of rules that answer class 1

$1:$ If $(\mathrm{a} 5 !=3) \wedge(\mathrm{a} 2 !=3) \wedge(\mathrm{a} 5 !=4)$ then $($ class $=1)$

2: If $(\mathrm{a} 2 !=3) \wedge(\mathrm{a} 5 !=4)$ then $($ class $=1)$

3: If $(\mathrm{a} 5 !=\mathrm{a} 4) \wedge(\mathrm{a} 4 !=\mathrm{a} 1) \wedge(\mathrm{a} 1 !=\mathrm{a} 3) \wedge(\mathrm{a} 4 !=2) \wedge(\mathrm{a} 4 !=\mathrm{a} 2) \wedge(\mathrm{a} 2 !=\mathrm{a} 6) \wedge(\$ \mathrm{C}=1)$

then $($ class $=1)$

4: If $(\mathrm{a} 5 !=\mathrm{a} 4) \wedge(\mathrm{a} 4 !=\mathrm{a} 1) \wedge(\mathrm{a} 5 !=4) \wedge(\mathrm{a} 1 !=\mathrm{a} 3) \wedge(\mathrm{a} 4 !=2)$ then $($ class $=1)$

The first rule is interesting because it is a more specialized version of the original second disjunctive component: [(A5 $\neq 4)$ and $(\mathbf{A 2} \neq 3)]$. Most likely the over-specialization is in place to remove instances with class noise, but the rule is still not $100 \%$ confident. 
The second rule is the same as the first but missing the term (a5 $!=3)$. Even though this exactly matches the second disjunctive component of the original definition it is not $100 \%$ confident because of the class noise. Interestingly, this rule takes advantage of its position in the hierarchy by first allowing rule \#1 to remove several instances that contain errors. By operating only on the remaining instances it allows for a greater overall confidence.

When examining the experiment history provided by EvRFind it was found that the first rule was $100 \%$ accurate on the test set, which means that the one record it incorrectly classified was probably class noise added to the training set. EvRFind had the following entry in the training log:

--> Mistake: Rule \#1 incorrect on record \#64.

- Rule- $>$ If $(\mathrm{a} 5 !=3) \wedge(\mathrm{a} 2 !=3) \wedge(\mathrm{a} 5 !=4)$ then $($ class $=1)$

-Record- $>$ class $=0$ a1 $=2$ a2 $=2$ a3 $=1$ a4=2 a5= $=2$ a6=1 id $=$ data $\_203$

According to the second disjunctive clause defining this problem, this record should have membership in class 1 , not class 0 as the record specifies. The corresponding record in the testing set is:

- Record- $>$ class $=1$ a1 $=2$ a2 $=2$ a3 $=1$ a4 $=2$ a5 $=2$ a6 $=1$ id $=$ data_ 203 
In this case, EvRFind was able to successfully ignore the class noise. The position of the rule in the hierarchy was maintained because of the high confidence and support, and this will always be the case when class noise is rare.

An example to the contrary was also observed in the case of rule \#2. Although rule \#2 performs very well, there are six cases in the testing set that it misclassifies.

- Rule- $>$ If $(\mathrm{a} 3 !=\mathrm{a} 4) \wedge(\mathrm{a} 2=3)$ then $($ class $=0)$

- Record- $>$ class $=1$ a1 $=1$ a2 $=3$ a3 $=2$ a4 $=1$ a5 $=3$ a6 $=1$ id $=$ data 125 -Record- $>$ class $=1$ a1 $=1$ a2 $=3$ a3 $=2$ a4 $=1$ a5 $=3$ a6 $=2$ id=data 126 - Record- $>$ class $=1$ a1 $=2$ a2 $=3$ a3 $=2$ a $4=1$ a5 $=3$ a6 $=1$ id $=$ data 269 - Record- $>$ class $=1$ a1 $=2$ a2 $=3$ a3 $=2$ a $4=1$ a5 $=3$ a6 $=2$ id $=$ data 270 - Record- $>$ class $=1$ a1 $=3$ a2 $=3$ a3 $=2$ a4 $=1$ a5 $=3$ a6 $=1$ id $=$ data 413 -Record- $>$ class $=1$ a1 $=3$ a2 $=3$ a3 $=2$ a4 $=1$ a5 $=3$ a6 $=2$ id=data 414

Table 7.7: Misclassified Monks-3 instances during one EvRFind experiment

Of the 30 instances answered by rule $\# 2$ in the training set, none of them were misclassified. There were a total of 90 correctly classified instances in the testing set. However, had one of the above counterexamples been present then this rule would likely have been modified and may not have survived in its current form.

Due to the high number of correctly classified instances by rule $\# 2$, the question arose as to whether EvRFind relied on erroneous instances during its discovery. To answer this question a manual set of rules was created and used in EvRFind to determine the actual instances that represented the class noise. This experiment will be more completely described below. 


\subsubsection{Evaluating Monks-3 with manual rules}

A set of manual rules were created and used as part of an experiment to determine how well EvRFind could ignore noise. The instances identified by EvRFind as containing class noise were found to be the following:

\begin{tabular}{|c|c|c|}
\hline \# & Training data & Testing data \\
\hline 5 & 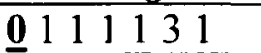 & 1111131 \\
\hline 61 & 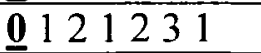 & 1121231 \\
\hline 203 & 0222212221 & 1221221 \\
\hline 213 & $\underline{0} 22213331$ & 1221331 \\
\hline 214 & $\underline{\mathbf{0}} 22213332$ & 1221332 \\
\hline 391 & 1331141 & $\begin{array}{lllllllllllll}0 & 3 & 3 & 1 & 1 & 4 & 4\end{array}$ \\
\hline
\end{tabular}

Table 7.8: Monks-3 instances containing class noise

The results of this experiment show that none of the noisy instances are answered, correctly or incorrectly, by rule \#2. Effectively, EvRFind has discovered a subset within class 0 that exists because the sampling of data used to create the training set was not representative of the entire domain.

\subsection{Chess end game: King+Rook versus King+Pawn}

\subsubsection{Description}

The Chess end-game (King+Rook versus King+Pawn) dataset is part of the UCI Repository [Blake, Murphy, 1998]. There are 3196 instances and each one uses 36 attributes to describe the locations of pieces on a chess board. Each instance also has a binary class that indicates whether the board is such that the "white" player can win the game or not. Approximately $52 \%$ belong to class 1 and $48 \%$ belong to class 2 . 


\subsubsection{Classification experiments and results}

In [Zheng, Webb, 2000] they describe a Lazy Bayes Rule (LBR) algorithm and compare its performance with several other learning algorithms using several datasets. One of the datasets used is the King+Rook versus King+Pawn, and those results are duplicated below.

\begin{tabular}{|l|c|l|}
\hline Algorithm / method & Accuracy & Source / Remarks \\
\hline Naïve Bayes & $87.8 \%$ & Zheng, Webb, 2000 \\
\hline C4.5 & $99.3 \%$ & Quinlan, 1993 \\
\hline BT (NBTree) & $98.5 \%$ & Kohavi, 1996 \\
\hline BSEJ & $97.3 \%$ & Pazzani, 1996 \\
\hline BSE & $93.5 \%$ & Pazzani, 1996; Kittler, 1986 \\
\hline LazyTree & $98.4 \%$ & Friedman et al., 1996; Zheng, Webb, 2000 \\
\hline LBR & $98.0 \%$ & Zheng, Webb, 2000 \\
\hline
\end{tabular}

Table 7.9: Published results for the King+Rook versus King+Pawn dataset

The best published result for this dataset is a predictive accuracy of $99.30 \%$, which was achieved by C4.5.

\subsubsection{Results with EvRFind}

Three sets of experiments were run with EvRFind. Each is comprised of ten runs using different random number seeds. The first used a training set of 1065 instances, which is approximately $30 \%$ of the data. The second experiment used 2876 instances, which is approximately $90 \%$ of the data. The final experiment used the entire training set to discover and refine rules. 


\begin{tabular}{|c|c|c|c|c|c|}
\hline TrSz & TsSz & Min \% & Max \% & AVG \% & Missed \\
\hline 1065 & 3196 & 72.78 & 81.98 & 75.08 & 30.10 \\
\hline 2876 & 3196 & 95.87 & 98.37 & 97.21 & 0.00 \\
\hline 3196 & 3196 & 95.84 & 98.31 & 97.59 & 0.00 \\
\hline
\end{tabular}

Table 7.10: Results of King+Rook versus King+Pawn dataset using EvRFind

Using only one third of the data resulted in a poor predictive model, although the performance appears to be related to the number of missed instances. As the number of example instances increases, the impact on performance due to instances that were missed during experimental evaluation was negated.

In the set of experiments where EvRFind used $90 \%$ of the data for training, which is comparable to other algorithms using a ten-fold cross validation, the performance was similar to that of the other learning algorithms. Similarly, when training on the full dataset the predictive accuracy was high, but this did not allow for evaluating the model on unseen data. The following two rules were discovered by EvRFind when training on the full dataset:

MV: \#H [(rimmx $=$ spcop) $]$

$[\mathrm{C} 99.78 \%, \mathrm{R}=890, \mathrm{~W}=2]$ :

If $($ hdchk $=$ bxqsq $) \wedge($ rxmsq $=$ qxmsq $) \wedge($ hdchk $!=$ wkpos $) \wedge($ rimmx $=$ wknck $) \wedge($ katri $!=1)$ then (class $=0)$

$[\mathrm{C} 100.00 \%, \mathrm{R}=782, \mathrm{~W}=0]:$ If $(\mathrm{bxqsq} !=\mathrm{stlmt}) \wedge(\# \mathrm{H})$ then $($ class $=1)$ 
In all of the experiments there were two dominant rules that covered a large percentage of each class. Although in this example there is a rule with $100 \%$ confidence that classifies a large number of instances, it was more often the case that both dominant rules were less than completely accurate. Most rules either had a small number of errors, or only classified a small percentage of instances.

\subsection{Tic Tac Toe Dataset}

\subsubsection{Description}

The Tic Tac Toe dataset is part of the UCI Repository. It contains 958 instances with 9 attributes used to describe a Tic Tac Toe board. The class attribute is binary and indicates whether the first player "X" has won the game or not. The dataset is free of noise and missing attributes.

\begin{tabular}{|c|c|c|}
\hline $\operatorname{TL}\{0,1,2\}$ & TM $\{0,1,2\}$ & $\operatorname{TR}\{0,1,2\}$ \\
\hline $\operatorname{ML}\{0,1,2\}$ & $\operatorname{MM}\{0,1,2\}$ & $\operatorname{MR}\{0,1,2\}$ \\
\hline $\operatorname{BL}\{0,1,2\}$ & $\operatorname{BM}\{0,1,2\}$ & $\operatorname{BR}\{0,1,2\}$ \\
\hline
\end{tabular}

\section{Table 7.11: Field description for the Tic Tac Toe dataset}

This table shows the representation of the Tic Tac Toe board, with values:

0 ; indicates a blank square

1 ; indicates that " $\mathrm{X}$ " occupies the square

2 ; indicates that " $\mathrm{O}$ " occupies the square

The class attribute value indicates:

0 ; that " $\mathrm{X}$ " has not won the game (either "O" won, or game is tied) 1 ; that "X" has won the game 
The set of instances is complete to the point where every possible outcome, considering that " $X$ " plays first, is represented.

\subsubsection{Classification experiments and results}

With a dataset that covers an entire domain using only a small number of instances, it is possible to fully evaluate the results of each learning algorithm. In the case of the Tic Tac Toe dataset, various training sets are used to create a predictive model that will be evaluated against the entire domain.

This domain has been the subject of many experiments, and together with the mushroom dataset they have become standard problems for evaluating learning systems [Muggleton, Bain, Hayes-Michie, Michie, 1989].

In the description file for the dataset, as well as in [Kokai, Toth, Zvada, 2002] and [Zheng, Webb, 2000], there are descriptions of several algorithms and their results. These are duplicated in the following table:

\begin{tabular}{|l|c|l|}
\hline Algorithm / Method & Accuracy & Source / Remarks \\
\hline CITRE & $76.7 \%$ & Matheus, Rendell, 1989 \\
\hline NewlD & $84.0 \%$ & Aha, 1991a \\
\hline CN2 & $98.1 \%$ & Aha, 1991a \\
\hline MBRtalk & $88.4 \%$ & Aha, 1991a \\
\hline IB1 & $98.1 \%$ & Aha, 1991a \\
\hline IB3 & $82.0 \%$ & Aha, 1991a \\
\hline IB3-C1 & $99.1 \%$ & Aha, 1991a \\
\hline NEWBOOLE-Card_4 & $99.5 \%$ & Sen, 1994 \\
\hline NEWBOOLE-Card_2 & $100.0 \% *$ & Sen, 1994 \\
\hline NB & $69.4 \%$ & Zheng, Webb, 2000 \\
\hline
\end{tabular}




\begin{tabular}{|l|l|l|}
\hline C4.5 & $86.3 \%$ & Quinlan, 1993 \\
\hline BT & $84.3 \%$ & Kohavi, 1996 \\
\hline BSEJ & $78.2 \%$ & Pazzani, 1996 \\
\hline BSE & $72.0 \%$ & Pazzani, 1996; Kittler, 1986 \\
\hline LazyTree & $95.5 \%$ & $\begin{array}{l}\text { Friedman et al., 1996; Zheng, } \\
\text { Webb, 2000 }\end{array}$ \\
\hline LBR & $86.5 \%$ & Zheng, Webb, 2000 \\
\hline
\end{tabular}

Table 7.12: Published results for the Tic Tac Toe dataset

The best result listed is for NEWBOOLE-Card_2, which is detailed in [Sen, 1994].

Unfortunately these results give domain specific knowledge to the learning algorithm by merging the values of ' $\mathrm{O}$ ' and blank, thus reducing the search space. The same algorithm achieves $99.5 \%$ when this data reduction step is not done, and therefore the summary will not include the artificially boosted value of $100 \%$ for this algorithm.

\subsubsection{Results with EvRFind}

Several experiments with EvRFind were performed on the Tic Tac Toe dataset. After the default values did not produce models that accurately covered $100 \%$ of the instances, several parameters were varied. Ten experiments were run for each of the parameter combinations listed in the table below.

\begin{tabular}{|l|c|c|c|c|c|c|c|}
\hline Options & TrSz & TsSz & Min \% & Max \% & AVG \% & Missed & Error \% \\
\hline MV+GF & 100 & 958 & 78.08 & 95.93 & 92.19 & 0.07808 & 7.75 \\
\hline MV & 100 & 958 & 73.17 & 81.11 & 78.64 & 0.21357 & 21.36 \\
\hline MV+GF & 671 & 287 & 93.03 & 99.30 & 96.38 & 0 & 3.62 \\
\hline MV & 671 & 287 & 97.56 & 99.65 & 98.71 & 0 & 1.29 \\
\hline MV+GF & 958 & 958 & 85.39 & 98.33 & 96.71 & 0 & 3.29 \\
\hline MV & 958 & 958 & 94.68 & 100.00 & 99.15 & 0 & 0.86 \\
\hline
\end{tabular}

Table 7.13: Initial EvRFind results for the Tic Tac Toe dataset 
Not taking into account the experiments where $100 \%$ of the data was used for training, EvRFind was still the best performer with a maximum predictive accuracy of $99.65 \%$ when training with $70 \%$ of the data. Although the NEWBOOLE-Card_2 algorithm did achieve $100 \%$ accuracy, it is not included in this comparison because the accuracy was boosted by supplying the algorithm with domain specific information.

The table shows that it was very rarely the case when instances were classified with $100 \%$ confidence. Not only did it require using the entire dataset for training, but the option to search for Gen-Functions had to be turned off. These results were not expected and warranted further analysis.

Example of rules discovered: 100 training instances

GF: $\$ A$ NEV $($ class $=$ General ANT $)=2\{$ Generalized $\}]$

GF: $\$ B$ NEV $($ class $=$ General ANT $)=3\{$ Variable $\}]$

GF: $\$$ E $[N E V($ class $=$ General ANT $)=2\{$ Constant $=1\}]$

$[\mathrm{C} 100.00 \%, \mathrm{~S} 68.00 \%, \mathrm{~A} 100.00 \%]:$ If $(\$ \mathrm{~A} !=\$ \mathrm{E})$ then $(\mathrm{Class}=1)$

$[\mathrm{C} 100.00 \%, \mathrm{~S} 25.00 \%, \mathrm{~A} 100.00 \%]:$ If $(\mathrm{BM} !=\mathrm{BL}) \wedge(\$ \mathrm{~A}=\$ \mathrm{E})$ then $(\mathrm{Class}=0)$

$[\mathrm{C} 100.00 \%, \mathrm{~S} 21.00 \%, \mathrm{~A} 19.05 \%]:$ If $(\mathrm{MR} !=\mathrm{BL}) \wedge(\$ \mathrm{~A}=\$ \mathrm{E})$ then $(\mathrm{Class}=0)$

$[\mathrm{C} 100.00 \%, \mathrm{~S} 16.00 \%, \mathrm{~A} 6.25 \%]:$ If $(\$ \mathrm{~A} !=\$ \mathrm{~B})$ then $(\mathrm{Class}=0)$

[C83.33\%,S70.00\%,A2.86\%]: If $(\$ A=\$ B)$ then $($ Class $=1)$

These rules achieved $92.80 \%$ confidence on the testing set. 
The discovered rules make use of Gen-Functions and attempt to classify the instances in a non-intuitive way. For example, the first rule translates to: "If the number of pairs of blanks or Os is not equal to the number of pairs of Xs, then X has won the game". Although this fact is true it is not intuitive to the human observer, in part because Tic Tac Toe defines a winning board configuration in more specific way. " $\mathrm{X}$ has won the game if three Xs exist in the same row, column, or diagonal".

Analysis of these rules shows that EvRFind was not searching for the answer to the question of whether " $\mathrm{X}$ has won the game", but rather the question of "what features are common when $\mathrm{X}$ is the winner". This is always the case with EvRFind, but in many experiments the rules are more obviously applicable to both questions.

The same experiment was run but EvRFind was not set up to create Gen-Functions. Instead of the five rules above, this experiment discovered that 20 rules were necessary, and resulted in a confidence of $80.27 \%$.

Building a predictive model with approximately $10 \%$ of the data was tried for comparison against other published results [Matheus, Rendel, 1989]. EvRFind compared favorably against CITRE, which achieved $76.7 \%$ accuracy using 100 instance training sets and 200 instance testing sets. 
Several other learning algorithms were used to build models based on approximately $70 \%$ training data [Aha, 1991a], [Sen, 1994]. To compare against these methods, the same experiments were run using EvRFind.

Example of rules discovered: $70 \%$ training (671 instances)

GF: $\$ A$ NEV(class $=$ General ANT $)=2\{$ Generalized $\}]$

GF: $\$ E[N E V($ class $=$ General ANT $)=3\{$ Variable $\}]$

GF: $\$$ I $[N E V($ class $=$ General ANT $)=4\{$ Variable $\}]$

$\mathrm{MV}: \# \mathrm{~F}[(\mathrm{TL} !=\mathrm{TR}) \wedge(\mathrm{BR} !=\mathrm{BL})]$

$[\mathrm{C} 100.00 \%, \mathrm{~S} 57.82 \%, \mathrm{~A} 100.00 \%]:$ If $(\$ \mathrm{I}=\$ \mathrm{E})$ then $($ Class $=1)$

$[\mathrm{C} 100.00 \%, \mathrm{~S} 33.38 \%, \mathrm{~A} 100.00 \%]$ : If $(\$ I !=1)$ then $($ Class $=0)$

[C96.87\%,S64.53\%,A10.39\%]: If $(\$ \mathrm{I}=1)$ then $($ Class $=1)$

$[\mathrm{C} 100.00 \%, \mathrm{~S} 7.45 \%, \mathrm{~A} 6.00 \%]:$ If $(\mathrm{BR} !=1) \wedge(\$ \mathrm{~A}=0) \wedge(\mathrm{MM}=\mathrm{TL}) \wedge(\mathrm{BL} !=\mathrm{TR})$ then

$($ Class $=0)$

$[\mathrm{C} 100.00 \%, \mathrm{~S} 5.51 \%, \mathrm{~A} 2.70 \%]$ : If $(\$ \mathrm{~A} !=1) \wedge(\mathrm{TL}=2) \wedge(\mathrm{BL} !=\mathrm{MM}) \wedge(\# \mathrm{~F}) \wedge(\mathrm{BM} !=\mathrm{TM})$

then $($ Class $=0)$

$[\mathrm{C} 97.01 \%, \mathrm{~S} 19.37 \%, \mathrm{~A} 2.31 \%]:$ If $(\mathrm{MM} !=1) \wedge(\$ \mathrm{~A}=0) \wedge(\mathrm{TL} !=\mathrm{BL})$ then $(\mathrm{Class}=0)$

$[\mathrm{C} 100.00 \%, \mathrm{~S} 6.86 \%, \mathrm{~A} 2.17 \%]:$ If $(\$ \mathrm{~A} !=1) \wedge(\mathrm{TL}=2) \wedge(\mathrm{BL} !=\mathrm{MM}) \wedge(\# \mathrm{~F}) \wedge(\mathrm{TL} !=\mathrm{TM})$

then $($ Class $=0)$

$[\mathrm{C} 93.48 \%, \mathrm{~S} 25.63 \%, \mathrm{~A} 1.74 \%]:$ If $(\mathrm{MM} !=1) \wedge(\$ \mathrm{~A}=0)$ then $($ Class $=0)$

$[\mathrm{C} 94.92 \%, \mathrm{~S} 16.69 \%, \mathrm{~A} 1.79 \%]:$ If $(\mathrm{BR} !=\mathrm{TL}) \wedge(\$ \mathrm{~A}=0) \wedge(\mathrm{BL} !=\mathrm{TR})$ then $($ Class $=0)$

$[\mathrm{C} 96.32 \%, \mathrm{~S} 19.52 \%, \mathrm{~A} 0.76 \%]:$ If $(\mathrm{MM} !=1) \wedge(\$ \mathrm{~A}=0) \wedge(\mathrm{ML} !=\mathrm{BR})$ then $($ Class $=0)$ 
These rules achieved $99.30 \%$ confidence on the testing set.

The first two rules appear to cover the majority of the dataset, and at first glance do not appear to reflect the basic rules of Tic Tac Toe. The first rule translates to: "If the number of unspecified four-of-a-kinds (blank, ' $\mathrm{X}$ ', or ' $\mathrm{O}$ ') is equal to the number of three-of-akinds (blank, ' $\mathrm{X}$ ', or ' $\mathrm{O}$ ' - but must be different from the four-of-a-kind), then ' $\mathrm{X}$ ' has won the game".

The second rule seems just as strange with the translation: "If there is not exactly one four-of-a-kind, whether it is blank squares, ' $\mathrm{X}$ 's, or ' $\mathrm{O}$ 's, then ' $\mathrm{X}$ ' has not won the game. Specifically, either ' $\mathrm{O}$ ' has won the game or the board is in a tied state."

When this experiment was tried without the option of creating Gen-Functions it yielded slightly better results on average, but many more rules were needed. For example, more than forty rules were required and the best one received much less support:

$\mathrm{MV}: \# \mathrm{~N}[(\mathrm{BR} !=2)]$

$[\mathrm{C} 100.00 \%, \mathrm{~S} 22.50 \%]:$ If $(\mathrm{TL} !=2) \wedge(\mathrm{MM} !=2) \wedge(\# \mathrm{~N})$ then $($ Class $=1)$

It translates to: "If the top-left square is not an ' $\mathrm{O}$ ', and the middle square is not an ' $\mathrm{O}$ ', and the bottom-right square is not an ' $\mathrm{O}$ ', then ' $\mathrm{X}$ ' has won the game'. 


\section{Varying search goals}

Because EvRFind was not able to discover rules to classify the entire dataset with $100 \%$ confidence, a manual set of rules was created to compare results with. They were immediately evaluated for confidence and support with the following results:

\section{$\underline{\text { Manual rules }}$}

$[\mathrm{C} 100.00 \%, \mathrm{~S} 8.14 \%]:$ If $(\mathrm{TL}=\mathrm{TM}) \wedge(\mathrm{TM}=\mathrm{TR}) \wedge(\mathrm{TR}=1)$ then $($ Class $=1)$

$[\mathrm{C} 100.00 \%, \mathrm{~S} 8.14 \%]:$ If $(\mathrm{ML}=\mathrm{MM}) \wedge(\mathrm{MM}=\mathrm{MR}) \wedge(\mathrm{MR}=1)$ then $($ Class $=1)$

$[\mathrm{C} 100.00 \%, \mathrm{~S} 8.14 \%]:$ If $(\mathrm{BL}=\mathrm{BM}) \wedge(\mathrm{BM}=\mathrm{BR}) \wedge(\mathrm{BR}=1)$ then $($ Class $=1)$

$[\mathrm{C} 100.00 \%, \mathrm{~S} 7.83 \%]:$ If $(\mathrm{TL}=\mathrm{ML}) \wedge(\mathrm{ML}=\mathrm{BL}) \wedge(\mathrm{BL}=1)$ then $($ Class $=1)$

$[\mathrm{C} 100.00 \%, \mathrm{~S} 7.83 \%]:$ If $(\mathrm{TM}=\mathrm{MM}) \wedge(\mathrm{MM}=\mathrm{BM}) \wedge(\mathrm{BM}=1)$ then $($ Class $=1)$

$[\mathrm{C} 100.00 \%, \mathrm{~S} 7.83 \%]:$ If $(\mathrm{TR}=\mathrm{MR}) \wedge(\mathrm{MR}=\mathrm{BR}) \wedge(\mathrm{BR}=1)$ then $(\mathrm{Class}=1)$

$[\mathrm{C} 100.00 \%, \mathrm{~S} 8.77 \%]:$ If $(\mathrm{TL}=\mathrm{MM}) \wedge(\mathrm{MM}=\mathrm{BR}) \wedge(\mathrm{BR}=1)$ then $(\mathrm{Class}=1)$

$[\mathrm{C} 100.00 \%, \mathrm{~S} 8.66 \%]:$ If $(\mathrm{TR}=\mathrm{MM}) \wedge(\mathrm{MM}=\mathrm{BL}) \wedge(\mathrm{BL}=1)$ then $(\mathrm{Class}=1)$

Records tested: 958

Coverage of correct answers: $626(65.34 \%)$

Total missed records: $332(34.66 \%)$

The missed records are labeled as such because EvRFind was not set to assume class 0 if other rules had failed. If one final rule, "Assume class 0", was in place then the dataset would be fully covered with $100 \%$ confidence. 
Considering that only a small number of rules are required to answer the entirety of class 1 , and EvRFind has the necessary grammar to express this, it was unclear as to why it couldn't achieve the goal. The only difference appeared to be the level of support held by the rules. On average, the manual rules have just over $8 \%$ support, while the best rule discovered by EvRFind has over 57\% support.

When discovering rules for classification tasks, EvRFind will attempt to maximize the confidence and support for each rule. This default behaviour is optional and these goals can be set manually. For the next set of experiments the desired confidence was left at $100 \%$, while the desired support was set to $8 \%$.

As a secondary experiment, some of these experiments were duplicated so that the hierarchy-protected-fitness option could be evaluated. In the following table, the HPF label indicates that the option was being used during training. These experiments will be summarized in section 7.13.3, Summary of experiments with hierarchy-protected-fitness, starting on page 281.

\begin{tabular}{|l|c|c|c|c|c|c|c|}
\hline Options & TrSz & TsSz & Min \% & Max \% & AVG \% & Missed & Error \% \\
\hline MV+GF & 671 & 287 & 96.17 & 98.95 & 97.28 & 2.1 & 1.99 \\
\hline MV+GF+HPF & 671 & 287 & 94.77 & 99.30 & 97.25 & 0.9 & 2.44 \\
\hline MV & 671 & 287 & 93.38 & 99.30 & 97.25 & 4.9 & 1.53 \\
\hline MV+HPF & 671 & 287 & 79.79 & 96.52 & 91.67 & 7.0 & 5.89 \\
\hline MV+GF & 958 & 958 & 99.48 & 100.00 & 99.78 & 0 & 0.22 \\
\hline MV & 958 & 958 & 98.02 & 100.00 & 99.75 & 0 & 0.25 \\
\hline
\end{tabular}

Table 7.14: EvRFind results using low-support for the Tic Tac Toe dataset

Interestingly, in the experiments where $100 \%$ of the training data was used, these results were better on average than those realized by the greedy counterpart that was striving for 
$100 \%$ support. Not reflected in the above table is how often the maximum accuracy was $100 \%$. In the first set of experiments that used the entire dataset for training, and also making use of Gen-Functions, there was one run where $100 \%$ accuracy was achieved. Although the average accuracy is lower for the second set of experiments, there were five runs that achieved $100 \%$ accuracy.

The hierarchies that attained $100 \%$ accuracy contain a large number of active rules. Many of the rules have a very low support, although each example contains several good rules as well. Following is an excerpt showing six rules from one of these hierarchies:

Example of low-support rules discovered

$\mathrm{MV}: \# \mathrm{C}[(\mathrm{TR}=1) \wedge(\mathrm{MM} !=2)]$

$\mathrm{MV}: \# \mathrm{D}[(\mathrm{MM} !=1) \wedge(\mathrm{BL}=2)]$

$M V: \# K[(T R=M R) \wedge(T R=B R)]$

$\mathrm{MV}: \# \mathrm{~S}[(\mathrm{TL}=\mathrm{BR}) \wedge(\mathrm{MM}=1)]$

MV: \#T [(TR != BR)]

MV: \#U [(TR = 2)]

$\mathrm{MV}: \# \mathrm{~V}[(\mathrm{TL} !=\mathrm{TR})]$

$M V: \# W[(B M=B L) \wedge(B M=B R)]$

$\mathrm{MV}: \# \mathrm{Y}[(\mathrm{BL}=\mathrm{MM}) \wedge(\# \mathrm{C})]$

MV: \#Z [(BM != TL)]

$\mathrm{MV}: \# \mathrm{AF}[(\# \mathrm{~S}) \wedge(\# \mathrm{~V})]$ 
$[\mathrm{C} 100.00 \%, \mathrm{~S} 7.93 \%]:$ If $(\mathrm{BR} !=2) \wedge(\mathrm{MR} !=\mathrm{MM}) \wedge(\# \mathrm{AF})$ then $($ Class $=1)$

$[\mathrm{C} 100.00 \%, \mathrm{~S} 6.78 \%]:$ If $(\# \mathrm{D}) \wedge(\# \mathrm{~T}) \wedge(\# \mathrm{~V})$ then $($ Class $=0)$

$[\mathrm{C} 100.00 \%, \mathrm{~S} 3.76 \%]$ : If $(\# \mathrm{~K}) \wedge(\# \mathrm{U})$ then $($ Class $=0)$

$[\mathrm{C} 100.00 \%, \mathrm{~S} 3.76 \%]:$ If $(\mathrm{TM}=\mathrm{TR}) \wedge(\mathrm{TR}=\mathrm{TL}) \wedge(\# \mathrm{U})$ then $($ Class $=0)$

$[\mathrm{C} 100.00 \%, \mathrm{~S} 7.41 \%]:$ If $(\mathrm{BR} !=2) \wedge(\# \mathrm{~W}) \wedge(\# \mathrm{Z})$ then $($ Class $=1)$

$[\mathrm{C} 100.00 \%, \mathrm{~S} 7.83 \%]:$ If $(\# \mathrm{Y}) \wedge(\# \mathrm{Z})$ then $($ Class $=1)$

\section{Greedy search goals}

The improved accuracy for experiments with the goals adjusted for lower support is an example of the pitfalls that are possible when using greedy algorithms. Greedy algorithms always make what appears to be the best choice at each stage without considering what happens afterwards. A greedy algorithm cannot go back to alter previous decisions, which means that only one path to the solution will be found. The only case where a greedy algorithm finds an optimal solution is when an optimal substructure exists within the search space, and many domains do not have this property.

While searching for the optimal Tic Tac Toe rules, EvRFind was using the greedy search goals of $100 \%$ confidence and $100 \%$ support. Although it is possible that EvRFind will find accurate rules with high support that cover the entire dataset, the experiments thus far have shown that following the non-greedy approach yields better results. 


\subsection{Polygon Dataset}

\subsubsection{Description}

The Polygon dataset was first described in [Cattral, Oppacher, Deugo, 1999], and was used to show that learning algorithms not capable of supporting relational learning were at a disadvantage in some domains.

\begin{tabular}{|l|l|}
\hline Attribute & Type / Information \\
\hline L1 & Integer $\{0-1500\}$ \\
\hline L2 & Integer $\{0-1500\}$ \\
\hline L3 & Integer $\{0-1500\}$ \\
\hline L4 & Integer $\{0-1500\}$ \\
\hline Class & Nominal $\{1,2,3,4,5\}$ \\
\hline
\end{tabular}

Table 7.15: Attribute description for the polygon dataset

There are four predictive attributes that describe, using an integer value, the length of one side of a polygon. It is possible to have a value of zero in at most one of the attributes, which would indicate that the polygon is some kind of triangle.

The training set contains 190 instances representing all five classes. The representation for each class varies with the majority class, isosceles triangle, making up almost $30 \%$ of the training set. The training set is not fully representative of the testing set, which contains 100,000 polygons equally distributed.

\begin{tabular}{|c|l|c|c|}
\hline$\#$ & Name and Description & Train \% & Test \% \\
\hline 1 & Equilateral triangle; three sides equal, one side zero & 18.95 & 20.07 \\
\hline 2 & Isosceles; two sides equal, one side non-zero, one side zero & 29.47 & 20.00 \\
\hline 3 & Scalene; three non-zero unique sides, one side zero & 25.26 & 19.98 \\
\hline 4 & Square; four equal sides & 13.16 & 19.85 \\
\hline 5 & Rectangle; two pairs of equal sides facing each other & 13.16 & 20.10 \\
\hline
\end{tabular}


Table 7.16: Class description for the polygon dataset

\subsubsection{Classification experiments and results}

In [Cattral, Oppacher, Deugo, 1999], RAGA ${ }^{1}$ was compared to C4.5 [Quinlan, 1993] and R [Becker, Chambers, Wilks, 1998] with the results duplicated below.

\begin{tabular}{|l|l|l|}
\hline Algorithm / Method & Accuracy & \# rules / Tree size \\
\hline RAGA (1999) & $93.09 \%$ & 19 rules \\
\hline C4.5 & $39.37 \%$ & 46 levels \\
\hline R & $30.27 \%$ & 17 terminal nodes \\
\hline
\end{tabular}

Table 7.17: Previous results on the Polygon dataset

The results of this work showed that the polygon dataset is difficult to classify. The range of possible values is large, and additionally it is important for the learning algorithm to discover relationships between attributes in order to produce good rules.

\subsubsection{Results using higher level constructs}

When EvRFind was allowed to use Meta-Variables and Gen-Functions, the Polygon dataset was classified more completely and accurately as compared to training without them.

As noted earlier, without the use of higher level constructs, RAGA was never able to achieve $100 \%$ confidence over the entire testing set. If the training set accurately represents the testing set, and if RAGA had been allowed to use very large rule

1 RAGA (Rule Acquisition with a Genetic Algorithm) was the predecessor to EvRFind and was a similar, but less developed, genetic-algorithm-based rule discovery system 
populations then it may have evolved perfect solutions eventually, but with a population limit in place it was not able to maintain the large number of low-support rules that were required. A basic example of this is the list of rules required to classify the Equilateral Triangle.

Manually created rules to classify an Equilateral Triangle

If $(\mathrm{L} 1=\mathrm{L} 2) \wedge(\mathrm{L} 2=\mathrm{L} 3) \wedge(\mathrm{L} 4=0)$ then $($ Class $=1)$

If $(\mathrm{L} 1=\mathrm{L} 2) \wedge(\mathrm{L} 2=\mathrm{L} 4) \wedge(\mathrm{L} 3=0)$ then $($ Class $=1)$

If $(\mathrm{L} 1=\mathrm{L} 3) \wedge(\mathrm{L} 3=\mathrm{L} 4) \wedge(\mathrm{L} 2=0)$ then $($ Class $=1)$

If $(\mathrm{L} 4=\mathrm{L} 2) \wedge(\mathrm{L} 2=\mathrm{L} 3) \wedge(\mathrm{L} 1=0)$ then $($ Class $=1)$

These four rules are used to represent a single concept, and many such rules would be required to correctly classify all instances as one of the five classes. With the option of using Gen-Functions, EvRFind is able to abstract structure from rules such as these. The result is that EvRFind always classifies the entire dataset with $100 \%$ confidence, and does so using rules like the following:

\section{General rules for classifying the polygon dataset}

GF: $\$$ A $[\mathrm{MNC}($ class $=$ General ANT $)]$

GF: $\$ B[N E V($ class $=$ General ANT $)=2\{$ Generalized $\}]$

GF: \$D [NEV(class $=$ General ANT $)=3\{$ Generalized $\}]$

GF: $\$ F[N E V($ class $=$ General ANT $)=4\{$ Generalized $\}]$

GF: $\$ H$ [RGC(class $=$ General ANT $)]$ 
If $(\$ B=1)$ then $($ Class $=2)$

If $(\$ F=\$ B) \wedge(\$ D<=\$ F)$ then $($ Class $=3)$

If $(\$ D=1)$ then $($ Class $=1)$

If $(\$ \mathrm{H}=1)$ then $($ Class $=4)$

If $(\$ B=2)$ then $($ Class $=5)$

In most cases EvRFind discovered exactly one rule to accurately cover each class. There were, however, some exceptions such as:

If $(\mathrm{L} 2<\mathrm{L} 3) \wedge(\mathrm{L} 2=\mathrm{L} 4)$ then $($ Class $=5)$

If $(\mathrm{L} 3=\mathrm{L} 1) \wedge(\mathrm{L} 4>\mathrm{L} 1)$ then $($ Class $=5)$

Unlike rules that address all instances of a given class, these two rules each answer a subset only. Depending on how difficult the class is to describe it may require several rules, including some that overlap in instances, to fully classify the proper examples with $100 \%$ accuracy.

\subsection{Balance-Scale}

\subsubsection{Description}

The Balance-Scale dataset is part of the UCI Machine Learning Repository and was originally described in [Siegler, 1976]. The dataset was generated to model psychological 
experimental results. Each instance contains four predictive attributes that are used to determine whether a scale is tipping to the left, or the right, or is balanced.

\begin{tabular}{|l|l|}
\hline Attribute & Type / Information \\
\hline Class & Nominal $\{0,1,2\}$ \\
\hline Left-weight & Nominal $\{1,2,3,4,5\}$ \\
\hline Left-distance & Nominal $\{1,2,3,4,5\}$ \\
\hline Right-weight & Nominal $\{1,2,3,4,5\}$ \\
\hline Right-distance & Nominal $\{1,2,3,4,5\}$ \\
\hline
\end{tabular}

Table 7.18: Attribute description for the Balance-Scale dataset

As can be seen in the table above, each of the four predictive attributes has exactly five possible values. With a testing set that contains 625 unique instances it means that the evaluation of the predictive model is going to fully describe the results because it is compared against every point in the domain.

\subsubsection{Classification experiments and results}

In [Zhang, Jiang, Su, 2005] the authors describe a Hidden Naïve Bayes (HNB) algorithm and compare its performance with several other learning algorithms using several datasets. One of the datasets used is the Balance-Scale, and those results are duplicated below.

\begin{tabular}{|l|l|l|}
\hline Algorithm / method & Accuracy \% & Source / Remarks \\
\hline C4.5 & $64.14 \pm 4.16$ & Quinlan, 1993 \\
\hline Naîve Bayes & $91.44 \pm 1.13$ & Langley, Iba, Thomas, 1992 \\
\hline SBC & $91.44 \pm 1.13$ & Langley, Sage, 1994 \\
\hline CL-TAN & $85.57 \pm 3.33$ & Friedman, Geiger, Goldszmidt, 1997 \\
\hline NBTree & $91.44 \pm 1.13$ & Kohavi, 1996 \\
\hline AODE & $89.78 \pm 1.88$ & Webb, Boughton, Wang, 2005 \\
\hline HNB & $89.14 \pm 2.05$ & Zhang, Jiang, Su, 2005 \\
\hline
\end{tabular}

Table 7.19: Published results for the Balance-Scale dataset 
The best results listed for this dataset, at $91.44 \% \pm 1.13 \%$ accuracy, were reported by three different algorithms. All of the experiments were performed using 10 -fold cross validation.

\subsubsection{Results with EvRFind}

EvRFind was evaluated using $90 \%$ of the data for training and the entire dataset for testing. The experiments were performed both with and without the hierarchy-protectedfitness option, which is summarized in section 7.13.3, Summary of experiments with hierarchy-protected-fitness, starting on page 281 .

\begin{tabular}{|l|c|c|c|c|c|c|c|}
\hline Options & TrSz & TsSz & Min \% & Max \% & AVG \% & Missed & Error \% \\
\hline HPF & 562 & 625 & 92.16 & 98.08 & 96.19 & 0 & 3.57 \\
\hline Non-HPF & 562 & 625 & 97.76 & 99.04 & 98.69 & 0 & 1.31 \\
\hline
\end{tabular}

Table 7.20: EvRFind results for the Balance-Scale dataset

In the best experiment, EvRFind was able to achieve $99.04 \%$ accuracy on this dataset.

This is a noticeable improvement over the best reported accuracy of $91.44 \% \pm 1.13 \%$.

\subsection{Breast Cancer}

\subsubsection{Description}

The Breast Cancer dataset is part of the UCI Machine Learning Repository and is one of three medical datasets provided by the Oncology Institute that are all commonly cited in machine learning literature. 
This dataset has nine predictive attributes that are either nominal or binary. There are a total of 286 instances that are classified as positive or negative for "recurrence events".

\subsubsection{Classification experiments and results}

In [Zhang, Jiang, Su, 2005] they describe a Hidden Naïve Bayes (HNB) algorithm and compare its performance with several other learning algorithms using several datasets. One of the datasets used is Breast-Cancer, and those results are duplicated below.

\begin{tabular}{|l|l|l|}
\hline Algorithm / method & Accuracy \% & Source / Remarks \\
\hline C4.5 & $75.26 \pm 5.04$ & Quinlan, 1993 \\
\hline Naïve Bayes & $72.94 \pm 7.71$ & Langley, Iba, Thomas, 1992 \\
\hline SBC & $72.53 \pm 7.52$ & Langley, Sage, 1994 \\
\hline CL-TAN & $67.95 \pm 6.8$ & Friedman, Geiger, Goldszmidt, 1997 \\
\hline NBTree & $71.66 \pm 7.92$ & Kohavi, 1996 \\
\hline AODE & $72.53 \pm 7.15$ & Webb, Boughton, Wang, 2005 \\
\hline HNB & $73.09 \pm 6.11$ & Zhang, Jiang, Su, 2005 \\
\hline
\end{tabular}

Table 7.21: Published results for the Breast-Cancer dataset

The best reported accuracy, $75.26 \pm 5.04$, was achieved by C4.5.

\subsubsection{Results with EvRFind}

EvRFind was evaluated using two sets of experiments on the Breast Cancer dataset. The first pair of experiments used $90 \%$ of the data for training, and evaluation of the model was done by using the entire dataset. The second set used $100 \%$ of the data for training and evaluation. 
As a secondary experiment, the hierarchy-protected-fitness option is evaluated by running some of the configurations twice, with only this option in difference. This experiment is included in the summary in section 7.13.3, Summary of experiments with hierarchy-protected-fitness, starting on page 281 .

\begin{tabular}{|l|c|c|c|c|c|c|c|}
\hline Options & TrSz & TsSz & Min \% & Max \% & AVG \% & Missed & Error \% \\
\hline HPF & 257 & 286 & 70.28 & 75.52 & 72.30 & 0.01 & 27.19 \\
\hline Non-HPF & 257 & 286 & 81.12 & 84.62 & 82.36 & - & 17.64 \\
\hline HPF & 286 & 286 & 70.28 & 70.63 & 70.40 & 0.01 & 28.52 \\
\hline Non-HPF & 286 & 286 & 84.27 & 87.76 & 86.09 & - & 13.91 \\
\hline
\end{tabular}

Table 7.22: EvRFind results for the Breast-Cancer dataset

The best result achieved by EvRFind when using less than $100 \%$ of the data for training was a predictive model with an accuracy of $84.62 \%$. This result is better than what is reported in other published work.

\subsection{Hill-Valley}

\subsubsection{Description}

The Hill-Valley problem is part of the UCI Machine Learning Repository. The problem contains four related datasets, as follows:

1) Hill-Valley without noise - Training

2) Hill-Valley without noise - Testing

3) Hill-Valley with noise - Training

4) Hill-Valley with noise - Testing 
Each of the four datasets has 606 instances, where there is no overlap between training and testing sets. There are 100 predictive attributes that, when combined with the attribute index, represent points on a two-dimensional graph. The class attribute indicates whether there is an incline towards a global maximum value (Hill) or a decline towards a global minimum value (Valley).

The following graph is a visual representation of a single training example, where it can be clearly seen that the class is "Valley".

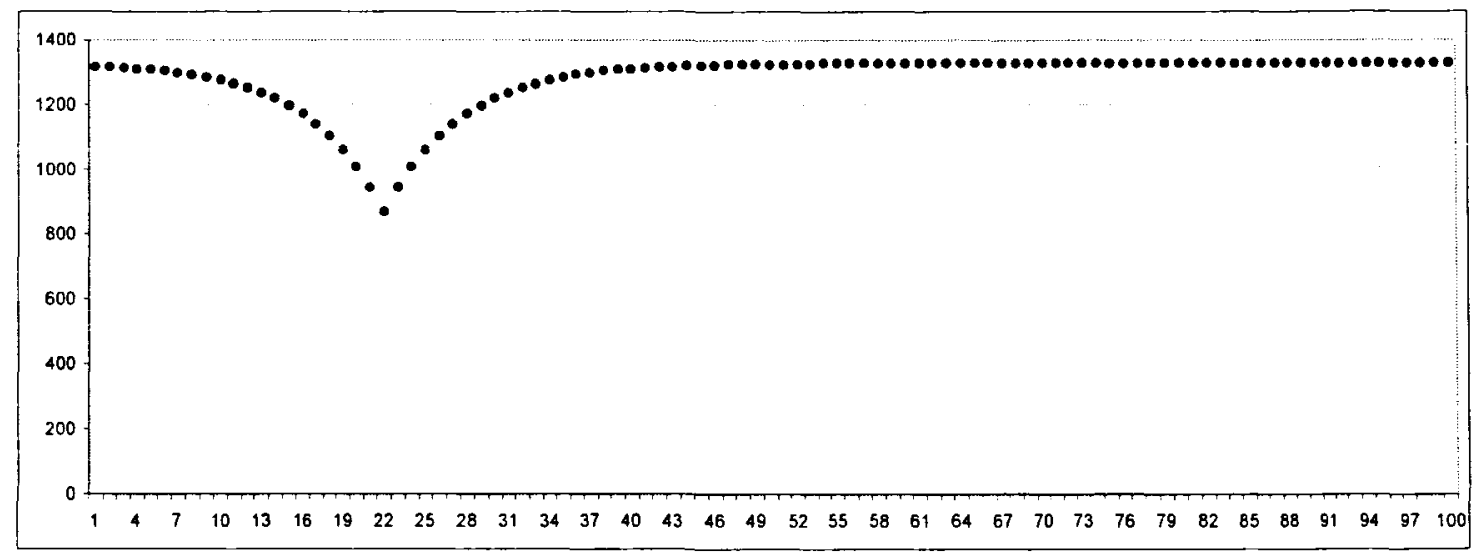

Figure 7.1: Example of 'valley' instance from Hill-Valley dataset without noise

In the dataset containing noise the minimum and maximum points are still visible, but the gradient is non-level. In the following graph, classified as a "Hill", the maximal point can still be seen, but the gradual incline is not as obvious between any adjacent pair of values. 


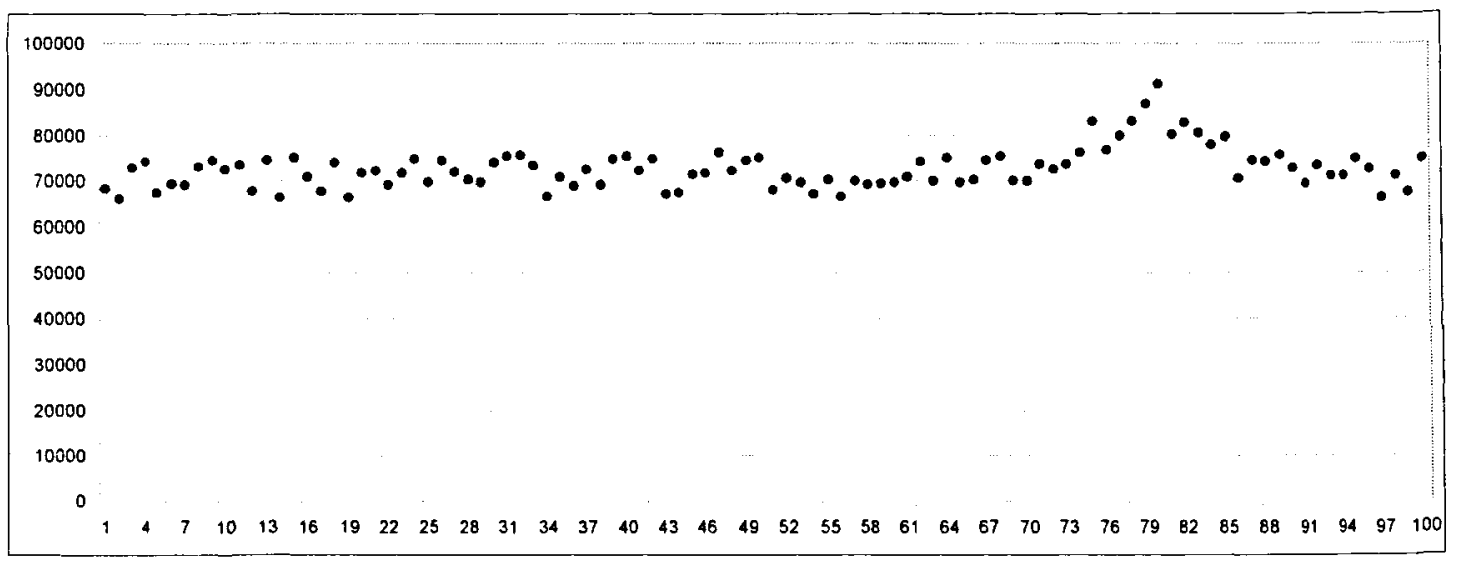

Figure 7.2: Example of 'hill' instance from Hill-Valley dataset with noise

\subsubsection{Classification experiments and results}

This problem was analyzed using a variety of algorithms in the Weka system. As with any binary classification task that has approximately $50 \%$ representation for each target, the goal is to create a model that achieves better than $50 \%$ accuracy. The following table indicates the predictive accuracy achieved on both the noisy and clean versions of the Hill-Valley problem:

\begin{tabular}{|l|c|c|}
\hline Algorithm & $\begin{array}{c}\text { Without noise } \\
(\%)\end{array}$ & $\begin{array}{c}\text { With noise } \\
(\%)\end{array}$ \\
\hline AdaBoostM1 & 48.680 & 51.485 \\
\hline Bagging & 60.396 & 57.591 \\
\hline BayesNet & 53.135 & 50.990 \\
\hline BayesNet & 48.680 & 49.340 \\
\hline ConjunctiveRule & 46.865 & 51.155 \\
\hline DecisionStump & 51.815 & 51.320 \\
\hline DecisionTable & 48.680 & 49.340 \\
\hline FilteredClassifier & 56.601 & 56.931 \\
\hline HyperPipes & 49.010 & 49.339 \\
\hline IBk & 61.881 & 56.930 \\
\hline J48 & 48.680 & 49.340 \\
\hline JRip & 54.125 & 52.475 \\
\hline KStar & 61.386 & 55.280 \\
\hline Logistic & $\mathbf{8 3 . 4 9 8}$ & $\mathbf{8 0 . 0 3 3}$ \\
\hline
\end{tabular}




\begin{tabular}{|l|c|c|}
\hline LWL & 52.475 & 51.485 \\
\hline MultilayerPerceptron & 52.970 & 53.465 \\
\hline NaiveBayes & 52.145 & 49.010 \\
\hline NBTree & 48.680 & 49.340 \\
\hline NNge & 58.251 & 50.495 \\
\hline PART & 48.680 & 49.340 \\
\hline RandomForest & 56.106 & 54.950 \\
\hline RandomTree & 53.135 & 51.320 \\
\hline RBFNetwork & 51.815 & 50.330 \\
\hline REPTree & 54.785 & 51.485 \\
\hline Ridor & 46.205 & 50.495 \\
\hline SMO & 55.116 & 53.465 \\
\hline VFI & 48.845 & 50.330 \\
\hline VotedPerceptron & 48.680 & 61.386 \\
\hline
\end{tabular}

\section{Table 7.23: Algorithm performance on the Hill-Valley datasets}

The average predictive accuracy for the algorithms in the Weka system was $53.62 \%$ for the dataset without noise, and $53.16 \%$ for the dataset with noise. The best algorithm, Logistic Regression, performed significantly better than the average with accuracies of $83.5 \%$ for the dataset without noise, and $80.03 \%$ for the dataset with noise.

\subsubsection{Results with EvRFind}

Experimental evaluation of EvRFind was performed using the same training and testing sets that were used for the Weka experiments.

As a secondary experiment, the hierarchy-protected-fitness option was also varied. The results for this are summarized in section 7.13.3, Summary of experiments with hierarchy-protected-fitness, starting on page 281. 


\begin{tabular}{|l|c|c|c|c|c|c|c|}
\hline Options & TrSz & TsSz & Min \% & Max \% & AVG \% & Missed & Error \% \\
\hline HPF & 606 & 606 & 99.01 & 100.0 & 99.60 & 0.0 & 0.33 \\
\hline Non-HPF & 606 & 606 & 99.34 & 100.0 & 99.60 & 0.0 & 0.28 \\
\hline
\end{tabular}

Table 7.24: EvRFind results for the Hill-Valley (no noise) dataset

The results attained by EvRFind in the dataset without noise were very good, and it was the top performer among those that were evaluated in this research. In many cases the predictive model was $100 \%$ accurate. An analysis of the results showed that EvRFind was able to determine relationships among "close" points, and use this information to determine whether the gradient was an incline or a decline.

\begin{tabular}{|l|c|c|c|c|c|c|c|}
\hline Options & TrSz & TsSz & Min \% & Max \% & AVG \% & Missed & Error \% \\
\hline HPF & 606 & 606 & 66.67 & 74.42 & 71.40 & 0.01 & 27.11 \\
\hline Non-HPF & 606 & 606 & 68.32 & 72.44 & 70.26 & 0.30 & 21.09 \\
\hline
\end{tabular}

Table 7.25: EvRFind results for the Hill-Valley (with noise) dataset

In the dataset with noise, EvRFind still performed better than average, but with the best accuracy of $74.24 \%$ it was unable to achieve the same results as the logistic regression algorithm in Weka at $80.03 \%$.

\subsection{Iris}

\subsubsection{Description}

The Iris dataset is part of the UCI Machine Learning Repository and is very commonly cited in the machine learning literature. This dataset contains three classes of 50 instances 
each. One of the classes is linearly separable from the other two, but the remaining two are not linearly separable from each other.

There are four real-valued predictive attributes, and the class is one of three different types of iris plant.

\subsubsection{Classification experiments and results}

Several datasets, including Iris, are compared using a number of algorithms in [Zhang, Jiang, Su, 2005] and [Zheng, Webb, 2000]. The results of these experiments are reproduced in the following table:

\begin{tabular}{|l|c|l|}
\hline Algorithm/Method & Accuracy & Source/ Remarks \\
\hline C4.5 & $96.00 \pm 4.64$ & Quinlan, 1993 \\
\hline Naïve Bayes & $94.33 \pm 6.79$ & Langley, Iba, Thomas, 1992 \\
\hline SBC & $96.67 \pm 4.59$ & Langley, Sage, 1994 \\
\hline CL-TAN & $91.73 \pm 8.16$ & Friedman, Geiger, Goldszmidt, 1997 \\
\hline NBTree & $95.27 \pm 6.16$ & Kohavi, 1996 \\
\hline AODE & $94.47 \pm 6.22$ & Webb, Boughton, Wang, 2005 \\
\hline HNB & $93.93 \pm 5.92$ & Zhang, Jiang, Su, 2005 \\
\hline BSEJ & 94.00 & Pazzani, 1996 \\
\hline BSE & 94.70 & Pazzani, 1996; Kittler, 1986 \\
\hline LazyTree & 93.70 & Friedman et al., 1996; Zheng, Webb, 2000 \\
\hline LBR & 93.70 & Zheng, Webb, 2000 \\
\hline
\end{tabular}

Table 7.26: Published results for the Iris dataset

The best reported accuracy, $96.67 \pm 4.59$, was achieved by SBC. 


\subsubsection{Results with EvRFind}

EvRFind was evaluated using two sets of experiments on the Iris dataset. The first pair of experiments used $50 \%$ of the data for training, and then the predictive model was evaluated using the entire dataset. The second set used $100 \%$ of the data for both training and evaluating the model.

As a secondary experiment, the hierarchy-protected-fitness option is evaluated by running some of the configurations twice, with only this option in difference. This experiment is included in the summary in section 7.13.3, Summary of experiments with hierarchy-protected-fitness, starting on page 281 .

\begin{tabular}{|l|c|c|c|c|c|c|c|}
\hline Options & TrSz & TsSz & Min \% & Max \% & AVG \% & Missed & Error \% \\
\hline HPF & 75 & 150 & 94.00 & 98.00 & 96.27 & 0.0 & 3.60 \\
\hline Non-HPF & 75 & 150 & 94.67 & 98.00 & 96.67 & 0.0 & 3.20 \\
\hline HPF & 150 & 150 & 96.00 & 97.33 & 97.06 & 0.0 & 2.97 \\
\hline Non-HPF & 150 & 150 & 96.00 & 98.00 & 96.93 & 0.0 & 3.07 \\
\hline
\end{tabular}

Table 7.27: EvRFind results for the Iris dataset

The best result achieved by EvRFind was a predictive model with an accuracy of $98.00 \%$. This result is approximately the same as what is reported for classification algorithms in other published work. 


\subsection{Glass}

\subsubsection{Description}

The Glass Identification dataset is part of the UCI Machine Learning Repository and was originally created to assist in the forensic identification of glass left at crime scenes. The data consists of 9 predictive attributes, all of which are real valued, that are used to predict one of seven different classes. There are 214 instances without noise or missing values.

\subsubsection{Classification experiments and results}

Several datasets, including Glass, are compared using a number of algorithms in [Zhang, Jiang, Su, 2005] and [Zheng, Webb, 2000]. The results of these experiments are reproduced in the following table:

\begin{tabular}{|l|c|l|}
\hline Algorithm / method & Accuracy \% & Source / Remarks \\
\hline C4.5 & $58.14 \pm 8.48$ & Quinlan, 1993 \\
\hline Naïve Bayes & $57.69 \pm 10.07$ & Langley, Iba, Thomas, 1992 \\
\hline SBC & $56.19 \pm 9.73$ & Langley, Sage, 1994 \\
\hline CL-TAN & $59.72 \pm 9.69$ & Friedman, Geiger, Goldszmidt, 1997 \\
\hline NBTree & $58.00 \pm 9.42$ & Kohavi, 1996 \\
\hline AODE & $61.12 \pm 9.79$ & Webb, Boughton, Wang, 2005 \\
\hline HNB & $59.02 \pm 8.67$ & Zhang, Jiang, Su, 2005 \\
\hline BSEJ & 68.00 & Pazzani, 1996 \\
\hline BSE & 67.30 & Pazzani, 1996; Kittler, 1986 \\
\hline LazyTree & 62.60 & Friedman et al., 1996; Zheng, Webb, 2000 \\
\hline LBR & 70.00 & Zheng, Webb, 2000 \\
\hline
\end{tabular}

Table 7.28: Published results for the Glass dataset

The best reported accuracy, $70 \%$, was achieved by LBR. 


\subsubsection{Results with EvRFind}

EvRFind was evaluated using two sets of experiments on the Glass dataset. The first pair of experiments used $90 \%$ of the data for training, and the results were evaluated using the entire dataset. The second set used $100 \%$ of the data for both training and evaluating the predictive model.

As a secondary experiment, the hierarchy-protected-fitness option is evaluated by running some of the configurations twice, with only this option in difference. This experiment is included in the summary in section 7.13.3, Summary of experiments with hierarchy-protected-fitness, starting on page 281.

\begin{tabular}{|l|c|c|c|c|c|c|c|}
\hline Options & TrSz & TsSz & Min \% & Max \% & AVG \% & Missed & Error \% \\
\hline HPF & 192 & 214 & 45.33 & 63.08 & 56.87 & 0.00 & 46.96 \\
\hline Non-HPF & 192 & 214 & 61.68 & 76.64 & 70.33 & 0.00 & 29.91 \\
\hline HPF & 214 & 214 & 44.86 & 63.08 & 56.87 & 0.00 & 43.13 \\
\hline Non-HPF & 214 & 214 & 67.29 & 79.44 & 72.01 & 0.00 & 28.14 \\
\hline
\end{tabular}

Table 7.29: EvRFind results for the Glass dataset

The best result achieve by EvRFind when using less than $100 \%$ of the data for training was a predictive model with an accuracy of $76.64 \%$. This result is slightly better than the best accuracy of $70 \%$ that was reported for classification algorithms in other published work. 


\subsection{Pima Indians Diabetes}

\subsubsection{Description}

The Pima Indians Diabetes dataset is part of the UCI Machine Learning Repository. All of the instances describe females that are least 21 years of age and of Pima Indian heritage.

This is a binary classification problem that uses 8 predictive attributes, of which there is a mix of integer and real values. There are 768 instances in the dataset that classify the Pima Indians as having diabetes or not.

\subsubsection{Classification experiments and results}

Several datasets, including Pima Indians, are compared using a number of algorithms in [Zhang, Jiang, Su, 2005] and [Zheng, Webb, 2000]. The results of these experiments are reproduced in the following table:

\begin{tabular}{|l|c|l|}
\hline Algorithm / Method & Accuracy & Source / Remarks \\
\hline C4.5 & $73.89 \pm 4.7$ & Quinlan, 1993 \\
\hline Naïve Bayes & $75.68 \pm 4.85$ & Langley, Iba, Thomas, 1992 \\
\hline SBC & $76.00 \pm 5.24$ & Langley, Sage, 1994 \\
\hline CL-TAN & $74.83 \pm 4.43$ & Friedman, Geiger, Goldszmidt, 1997 \\
\hline NBTree & $75.28 \pm 4.84$ & Kohavi, 1996 \\
\hline AODE & $76.37 \pm 4.35$ & Webb, Boughton, Wang, 2005 \\
\hline HNB & $76.00 \pm 4.6$ & Zhang, Jiang, Su, 2005 \\
\hline BSEJ & 74.70 & Pazzani, 1996 \\
\hline BSE & 74.60 & Pazzani, 1996; Kittler, 1986 \\
\hline LazyTree & 71.80 & Friedman et al., 1996; Zheng, Webb, 2000 \\
\hline LBR & 74.60 & Zheng, Webb, 2000 \\
\hline
\end{tabular}

Table 7.30: Published results for the Pima Indians dataset 
The best reported accuracy, $76.37 \% \pm 4.35 \%$, was achieved by AODE.

\subsubsection{Results with EvRFind}

EvRFind was evaluated using two sets of experiments on the Iris dataset. The first pair of experiments used $90 \%$ of the data for training, and the entire dataset was used for evaluating the predictive model. The second set used $100 \%$ of the data for both training and evaluation.

As a secondary experiment, the hierarchy-protected-fitness option is evaluated by running some of the configurations twice, with only this option in difference. This experiment is included in the summary in section 7.13.3, Summary of experiments with hierarchy-protected-fitness, starting on page 281.

\begin{tabular}{|l|c|c|c|c|c|c|c|}
\hline Options & TrSz & TsSz & Min \% & Max \% & AVG \% & Missed & Error \% \\
\hline HPF & 690 & 768 & 64.06 & 74.74 & 71.62 & 0.01 & 27.34 \\
\hline Non-HPF & 690 & 768 & 83.85 & 87.76 & 86.16 & 0.00 & 13.89 \\
\hline HPF & 768 & 768 & 62.50 & 75.78 & 71.67 & 0.00 & 27.67 \\
\hline Non-HPF & 768 & 768 & 84.64 & 89.97 & 86.85 & 0.00 & 13.18 \\
\hline
\end{tabular}

Table 7.31: EvRFind results for the Pima Indians Diabetes dataset

The best result achieve by EvRFind when using less than $100 \%$ of the data for training was a predictive model with an accuracy of $87.76 \%$. This result is better than what is reported for classification algorithms in other published work. 


\subsection{Experimental Evaluation Summary}

\subsubsection{Summary of EvRFind results}

Shown in the following table are the predictive accuracy (minimum, maximum and average), average number of missed instances and average rate of error for the combined runs of each dataset. Only the "best-run", which can be identified by the options, for each problem is shown. This table does not include results for experiments that were performed using $100 \%$ of the data for training.

\begin{tabular}{|l|l|c|c|c|c|}
\hline Problem & Options & Min \% & Max \% & AVG \% & Missed \\
\hline Mushroom & Training size $\sim 50 \%$ & 99.41 & 99.95 & 99.79 & 0.00 \\
\hline Monks-1 & Default & 100.0 & 100.0 & 100.0 & 0.00 \\
\hline Monks-2 & Default & 93.75 & 100.0 & 99.03 & 0.00 \\
\hline Monks-3 & Default & 94.21 & 98.61 & 97.01 & 0.00 \\
\hline KR vs KP & Training size $\sim 90 \%$ & 95.87 & 98.37 & 97.21 & 0.00 \\
\hline Tic Tac Toe & MV Only & 97.56 & 99.65 & 98.71 & 0.00 \\
\hline Polygon & Default & 100.0 & 100.0 & 100.0 & 0.00 \\
\hline Balance-Scale & Default & 97.76 & 99.04 & 98.69 & 0.00 \\
\hline Breast Cancer & Training size $\sim 90 \%$ & 81.12 & 84.62 & 82.36 & 0.00 \\
\hline Hill-Valley & Default & 99.34 & 100 & 99.60 & 0.00 \\
\hline $\begin{array}{l}\text { Hill-Valley } \\
\text { with noise }\end{array}$ & Hierarchy protected fitness & 66.67 & 74.22 & 71.40 & 0.00 \\
\hline Iris & Training size $\sim 50 \%$ & 96.00 & 98.00 & 96.93 & 0.00 \\
\hline Glass & Training size $\sim 90 \%$ & 61.68 & 76.64 & 70.33 & 0.00 \\
\hline Pima Indians & Training size $\sim 90 \%$ & 83.85 & 87.76 & 86.16 & 0.00 \\
\hline
\end{tabular}

Table 7.32: EvRFind evaluation summary for popular datasets

The table, and the corresponding chart, shows the summary of the accuracy achieved on public datasets that were analyzed using EvRFind for this research. 


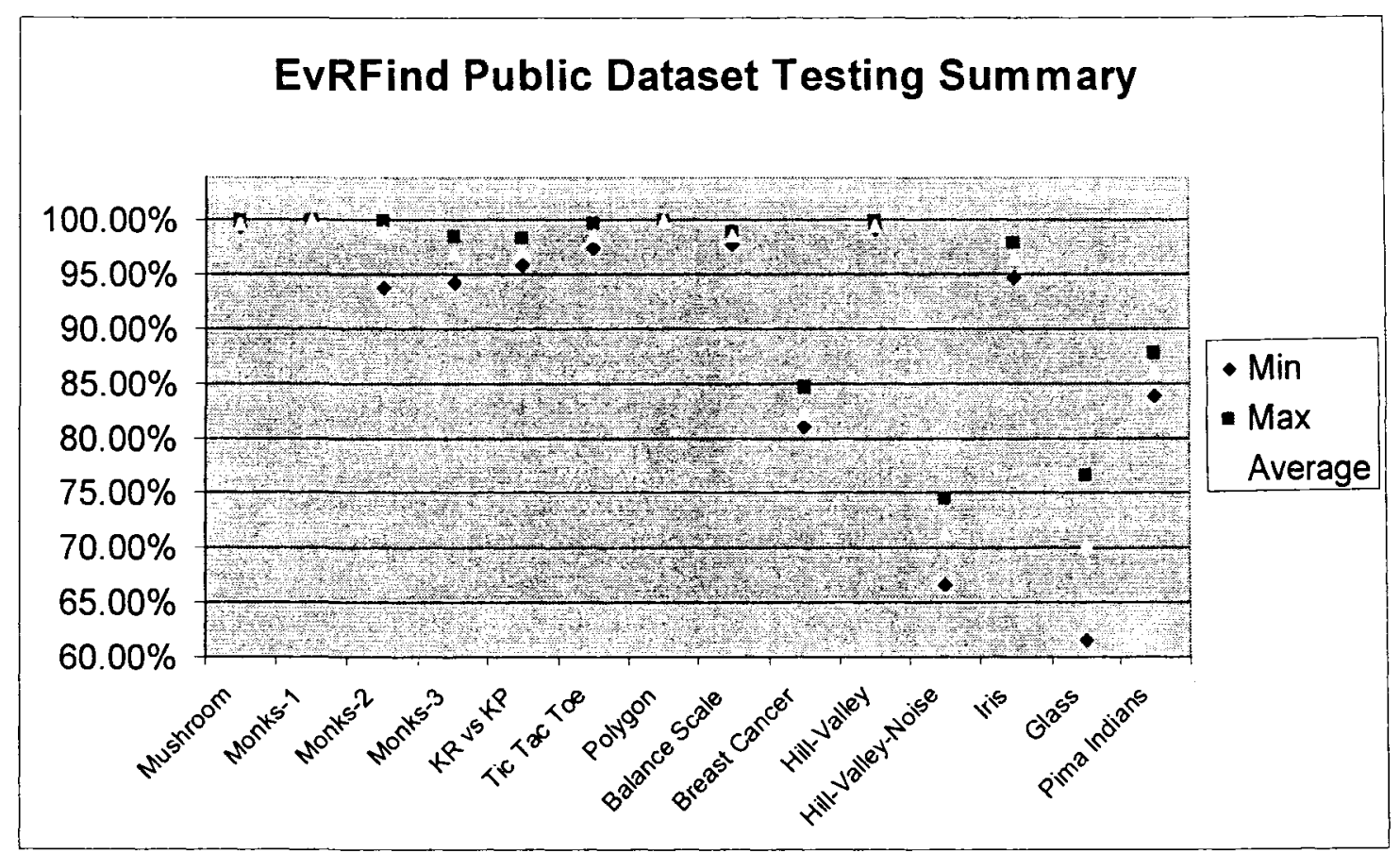

Figure 7.3: EvRFind performance on public datasets

\subsubsection{Summary of comparative analysis}

The following table compares the best results achieved by EvRFind against the best published results for other algorithms. Shown is the maximum predictive accuracy achieved for both algorithms, as well as the name of the best-of algorithm for the problem.

\begin{tabular}{|l|c|c|l|}
\hline Problem & EvRFind \% & Other \% & \multicolumn{1}{c|}{ Other Algorithm } \\
\hline Mushroom & 99.95 & 100.0 & C-MLP2LN \\
\hline Monks-1 & 100.0 & 100.0 & C-MLP2LN \\
\hline Monks-2 & 100.0 & 100.0 & C-MLP2LN \\
\hline Monks-3 & 98.61 & 100.0 & C-MLP2LN \\
\hline KR vs KP & 98.37 & 99.30 & C4.5 \\
\hline Tic Tac Toe & 99.65 & 99.50 & NEWBOOLE-Card_2 \\
\hline Polygon & 100.0 & 39.37 & C4.5 \\
\hline Balance-Scale & 99.04 & $91.44 \pm 1.13$ & Naïve Bayes, SBC, NBTree \\
\hline Breast Cancer & 84.62 & $75.26 \pm 5.04$ & C4.5 \\
\hline
\end{tabular}




\begin{tabular}{|l|c|c|l|}
\hline Hill-Valley & 100.0 & 83.50 & Logistic regression (Weka) \\
\hline $\begin{array}{l}\text { Hill-Valley } \\
\text { with noise }\end{array}$ & 74.42 & 80.03 & Logistic regression (Weka) \\
\hline Iris & 98.00 & $96.67 \pm 4.59$ & SBC \\
\hline Glass & 76.64 & 70.00 & LBR \\
\hline Pima Indians & 87.76 & $76.37 \pm 4.35$ & AODE \\
\hline
\end{tabular}

Table 7.33: Comparison of results for popular datasets

Over all of the problems listed in this table, the average predictive accuracy for models created by EvRFind is $94.08 \%$. Referring to published work for the same datasets, the average for the best combination of competing algorithms in each dataset is $86.53 \%$. Although EvRFind was not the top performing system for every dataset, there was not a single technique that achieved a higher predictive accuracy in every domain.

Of the datasets that were used during the experimental evaluate phase, the results of EvRFind varied considerably with respect to other algorithms. In some cases such as the Hill Valley with Noise and Monks-3 datasets, EvRFind was outperformed by at least one algorithm. In other domains such as Polygon, Balance-Scale, Breast Cancer, Hill-Valley without Noise, Glass, and Pima Indians Diabetes, EvRFind achieved the best predictive accuracy. Finally, in the Mushroom dataset, KR vs KP, the first two Monks problems, Tic Tac Toe, and the Iris dataset, EvRFind performed with similar success as compared to the best competing algorithm. 


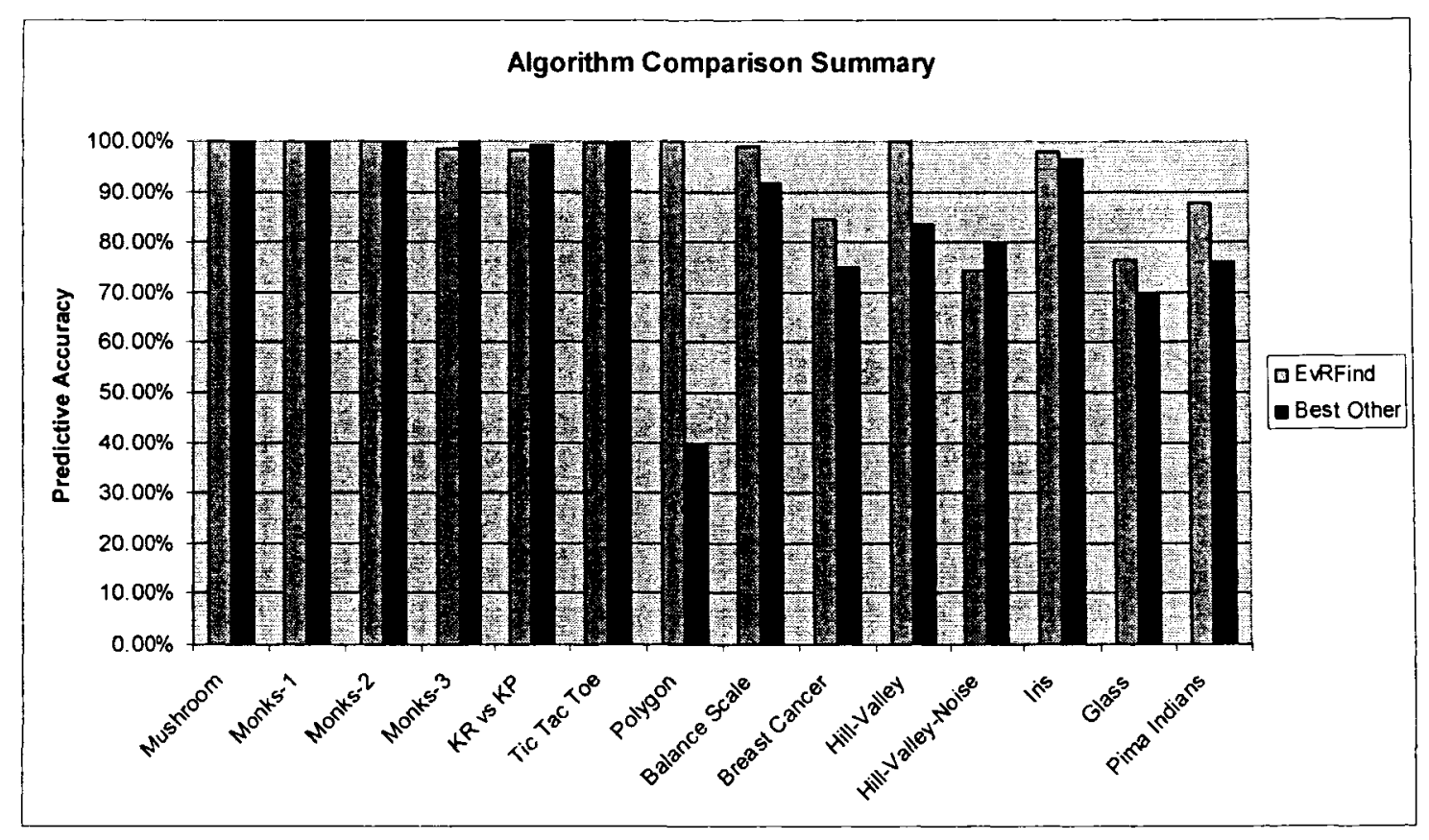

Figure 7.4: Comparison of EvRFind to best competing algorithm

Based on these results it would appear that EvRFind performs best when it is able to take advantage of generalization, as it does in the case of the Polygon dataset. Conversely, the predictive models generated by EvRFind do not perform as well when the domain contains noise. This was most obvious in the third Monks problem, which contained 5\% class noise, as well as in the Hill-Valley domain when attribute noise was present.

\subsubsection{Summary of experiments with hierarchy-protected-fitness}

Evaluation of the hierarchy-protected-fitness option was done on several of the public datasets described in this chapter. The following table summarizes the results from these experiments.

\begin{tabular}{|l|l|c|c|c|}
\hline Dataset & Options & HPF & AVG \% & Missed \\
\hline Tic Tac Toe & MV + GF + Low support & Yes & 97.25 & 0.9 \\
\hline
\end{tabular}




\begin{tabular}{|c|c|c|c|c|}
\hline Tic Tac Toe & $\mathrm{MV}+\mathrm{GF}+$ Low support & No & 97.28 & 2.1 \\
\hline Tic Tac Toe & MV only + Low support & Yes & 91.67 & 7.0 \\
\hline Tic Tac Toe & MV only + Low support & No & 97.25 & 4.9 \\
\hline Balance-Scale & & Yes & 96.19 & - \\
\hline Balance-Scale & & No & 98.69 & - \\
\hline Breast Cancer & Training size $\sim 90 \%$ & Yes & 72.30 & 0.01 \\
\hline Breast Cancer & Training size $\sim 90 \%$ & No & 82.36 & 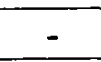 \\
\hline Breast Cancer & Training size $\sim 100 \%$ & Yes & 70.40 & 0.01 \\
\hline Breast Cancer & Training size $\sim 100 \%$ & No & 86.09 & - \\
\hline Hill-Valley & & Yes & 99.60 & - \\
\hline Hill-Valley & & No & 99.60 & - \\
\hline Hill-Valley with noise & & Yes & 74.42 & 0.01 \\
\hline Hill-Valley with noise & & No & 72.44 & 0.30 \\
\hline Iris & Training size $\sim 50 \%$ & Yes & 96.27 & - \\
\hline Iris & Training size $\sim 50 \%$ & No & 96.67 & - \\
\hline Iris & Training size $\sim 100 \%$ & Yes & 97.06 & - \\
\hline Iris & Training size $\sim 100 \%$ & No & 96.93 & - \\
\hline Glass & Training size $\sim 90 \%$ & Yes & 56.87 & - \\
\hline Glass & Training size $\sim 90 \%$ & No & 70.33 & - \\
\hline Glass & Training size $\sim 100 \%$ & Yes & 56.87 & - \\
\hline Glass & Training size $\sim 100 \%$ & No & 72.01 & - \\
\hline Pima Indians & Training size $\sim 90 \%$ & Yes & 71.62 & 0.01 \\
\hline Pima Indians & Training size $\sim 90 \%$ & No & 86.16 & - \\
\hline Pima Indians & Training size $\sim 100 \%$ & Yes & 71.67 & - \\
\hline Pima Indians & Training size $\sim 100 \%$ & No & 86.85 & - \\
\hline
\end{tabular}

Table 7.34: Summary of miscellaneous hierarchy-protected-fitness experiments

As can be seen in the previous table, the use of the hierarchy-protected-fitness option very rarely creates a more accurate predictive model. The average predictive accuracy for these experiments without using the option is $87.90 \%$. When the option is turned on and the system makes an extra effort to build a default hierarchy, the average drops to $80.94 \%$.

Based on this empirical evidence is can be concluded that forcing the system to explicitly take the hierarchy into consideration during training will often negatively impact the performance. 
A more in-depth analysis of the results has revealed that the rules generated by this option suffer more from overfitting. Also worth noting is that the search process was concluded faster. Although this would be advantageous if the results were similar, the reduced search time is quite likely one of the reasons for the reduction in overall predictive accuracy.

\subsubsection{Summary of experiments for the lifetime duplicate check}

At the commencement of evaluating EvRFind using publicly available datasets it was decided that each experiment would be run twice, both with and without the lifetime duplicate check option enabled. Similarly to the evaluation process for the hierarchy protected fitness option, it was expected that the empirical results would indicate whether the technique was beneficial.

Not surprisingly, after evaluating the option several times on the Mushroom dataset, Polygon dataset, and the Poker Hand dataset, it was determined that the option was consistently beneficial in terms of creating the most accurate predictive models. Consequently, the use of this option became standard and the comparative analysis was not completed for all datasets.

The disadvantage to using the lifetime duplicate check option is that additional overhead is incurred by the maintenance of the entire ancestral history. However, the efficient way in which this is implemented helps to curb this negative effect. 
One additional benefit that occurs as a side effect is that a more in-depth analysis of the search results can be performed. This is possible because a great deal more information about individuals within populations, and what occurred during the evolution from each generation to the next, is stored in order to maintain the history. Although this was not used heavily during this research, it may be useful for future tasks in yet unseen domains. 


\section{Chapter 8: Conclusion}

This research has developed a rule discovery tool that combines and expands upon other highly reliable techniques used in the fields of data mining, machine learning, and evolutionary computing. Through the design, implementation, and evaluation of EvRFind, "Evolutionary Rule Finder", this research satisfies the goals as set forth at the beginning of this thesis.

Among the contributions in this work are advancements in the fields of genetic algorithms and machine learning. These contributions, as well as the milestone objectives that were required to achieve them, are summarized below.

\subsection{Summary of Contributions}

EvRFind is an effective rule discovery tool that creates predictive models in the form of default hierarchies. The default hierarchy effectively stems the problem of over-fitting because there is no pressure to make a trade-off between predictive accuracy and maintaining more general rules.

At the commencement of this research, one of the unanswered questions concerned the formation of the default hierarchy during the evolutionary search. Specifically, would it be beneficial to have the system explicitly aim to create a default hierarchy, or is it sufficient to rely on the self-organization that emerges throughout the entire search? 
It was determined empirically that attempts to force the creation of an ordered hierarchy, where rules depend on others for protection, led to premature over-fitting. This result was in direct contrast to the goal, and consequently it was determined that self-organization of the rules was the more robust solution. Although there may be domains where explicit direction to the ordering of a hierarchy can be of benefit, no such examples were found during this research.

EvRFind originally started as a purely genetic-algorithm-based rule discovery algorithm that did very little during the intergenerational processing stage. Benefits were quickly realized as additional functionality was added to this phase of the search. In particular, significant progress was realized with the implementation of the language extending components, Meta-Variables and Gen-Functions. These are both examples of "on the fly" constructive induction.

The implementation of the Meta-Variable allows for the identification of reliable and frequently occurring terms within the set of rules. Once identified, the significant terms are encapsulated and the representation language is extended accordingly. This process is somewhat similar to the concept of learning from experience.

Experiments with Meta-Variables have shown that their use facilitates the search process through the more rapid dissemination of these logical stepping stones. This success is apparent in different areas, and in particular, evidence of concentrating mutation on 
unproven parts of an individual rule was shown to have had greatly increased the search efficiency.

The implementation of the Gen-Function allows for the automatic generalization of rules based on primitive functions that are stored in an internal library. After a common theme between rules is detected, the system attempts to analyze and more deeply understand the similarity. This is similar to the notion of identifying a new concept. If a definite pattern can be verified then the language is extended accordingly.

Experimenting with the Gen-Function component has shown significant benefit in several domains. Most importantly, the Gen-Function will identify non-linear relationships between attributes. These relationships are sometimes unpredictable and even counterintuitive, which means these patterns are unlikely to have been discovered using conventional means.

Hundreds of experiments were run to evaluate the effect of the language-extending capability offered by Meta-Variables and Gen-Functions. Due to the success observed through the use of these non-evolutionary components, both of these options have become standard and are now used by default. Although it has been noted that some domains do not take advantage of this functionality, experimentation has shown that the search process and resulting predictive models are not negatively affected in these cases. 
Aside from the optimization and language-extending functionality that is performed between generations, EvRFind employs a local search technique, Hill Climbing, in an attempt to fine tune potential solutions. This non-evolutionary search technique is normally very computationally intensive, but this is curbed by the fact that evolution is used to point the search in the right direction.

The effect of the Hill Climbing search is similar to performing a variety of mutations on a single rule during a single generation. This has been shown to effectively explore the search space surrounding a specific rule, and take advantage of minor tweaks that are less likely to be discovered during the normal course.

One of the intermediate objectives was to implement, test and evaluate the concept of a lifetime duplicate check. This was first considered because the existing technique of removing duplicate solutions was restricted to the population of a single generation. Although this had a marked improvement over systems that did not eliminate duplicate solutions, the problem of solution re-discovery was eventually detected during the development and evaluation phases.

A comparative analysis was performed using the lifetime duplicate check, and while it could not be said that it always improved the search efficiency, it was shown to have done so in most cases. Furthermore, there was not an observed case where the exploratory part of the search was negatively impacted. Consequently, the lifetime 
duplicate check was made part of the standard configuration and is now used by default during the search.

Specific to the task of classification in machine learning, functionality for the automatic redirection of computing resource was implemented. This is known as automatic consequent balancing. This component effectively re-directs the search away from well understood concepts and re-focuses on areas of the domain that are less understood.

The evaluation of EvRFind was done in two ways; first, predictive models were built for publicly available datasets and the results were compared to other published work. Second, a difficult synthetic dataset was developed and subjected to variety of experiments with EvRFind and other publicly available classification algorithms.

On the task of creating predictive models for publicly available datasets and comparing them against published work, EvRFind produced results that had a higher predictive accuracy, on average, than the best competing algorithms. Although EvRFind was outperformed by some algorithms in certain domains, there was not one particular algorithm that consistently achieved better predictive accuracy over all domains.

EvRFind compared favourably against many of the algorithms for which results have been published. In particular, the greatest improvement was observed in datasets where EvRFind was able to generalize concepts. Conversely, EvRFind did not perform as well when the domain contained noise. 
The creation of the poker hand dataset is also a contribution to the fields of data mining and machine learning. This synthetic dataset, which is now part of the UCI Machine Learning Repository, represents a very large, imbalanced, and challenging domain. There are several target concepts, each with varying distribution. Also contributing to the difficulty is the chosen representation, and that some target classes are subsets of others. In most cases there is overlap in the subclasses where subtle differences make the distinction between them. Different target classes rely on different combinations of predictive attributes, which means that feature selection is of limited use.

EvRFind performed favourably when compared to the other classification algorithms that were used to perform experiments on this dataset. With the best model yielding a predictive accuracy of $99.96 \%$ coverage, it is expected that with the inclusion of new Gen-Function primitives, future versions of EvRFind will achieve 100\% coverage for this difficult domain.

\subsection{Future Work}

Throughout the implementation and evaluation of EvRFind there were a number of areas that were noted as having potential for expansion or refinement in future versions. Some examples of these follow. 


\subsubsection{Dataset specific constant values}

The introduction of experiment-wide constants, specifically related to the dataset, can be easily compiled and may be of use in some domains. Some potentially useful examples of constant values are:

1) "attribute_X_minimum_value"; this constant would represent the minimum value for the specified "X" attribute in the dataset. This value would be determined after scanning the data during the pre-processing stage. The computational effort is already committed by the DFS module, but the value is currently being used for reporting purposes only.

2) "attribute_X_lower_bound"; this constant would represent the lower bound for the attribute, as specified by the user during the configuration stage.

3) "attribute_X_mean"; this constant represents the average value for the attribute. As with other interesting calculations, such as the attribute median or mode values, this work is not currently being done but could easily be incorporated into the pre-processing stage.

The inclusion of constant values such as these would make it possible for rules to take advantage of meta-knowledge that may not normally be available. As an example, the following rule could be discovered: 
If $($ Size $=$ attribute_size_lower_bound $)$ Then (Class = small)

Instead of:

If $($ Size $=2)$ Then $($ Class $=$ small $)$

Although a trivial example, expressing this same relationship is more general than using a constant value. Particularly in cases where the bounds might change over time, the rule that uses the global constant will be more robust.

\subsubsection{Additional Gen-Function primitives}

Experiments up to this point have shown that the use of Gen-Functions is beneficial, and to allow for maximum flexibility it is important to include as many primitive GenFunctions as possible. Because the selection process relies on evolution to limit the scope, the number of primitives does not over-burden the search. A list of Gen-Functions that are planned for the next version of EvRFind are available in Appendix G: Future Gen-Function Primitives, on page 313.

\subsubsection{General language extension improvements}

\section{Fitness}

Including a fitness for a specific Meta-Variable or Gen-Function will enable the system to monitor how good or how useful each one is. In the current implementation, all Meta- 
Variables and Gen-Functions are considered equally during the selection process. When the fitness measure is implemented, this will be used to bias the probabilistic nature of the selection and usage of these language extensions.

\section{Review Process}

Once a Meta-Variable or Gen-Function is created, it stays in the system until the experiment is finished. In some cases these extensions become redundant and a superior version should be used instead. If an "extinction" flag is implemented then a review process will periodically assess the value of each new component. If one is found to be redundant then it can be marked as extinct, which means it can still be evaluated but not selected for use in newly generated or modified terms.

\subsubsection{Post-search optimization of rules}

Another addition that is planned for the next version of EvRFind is a rule postevolutionary optimization phase. This will examine rules in search of patterns that are suitable for replacement with a more optimized version. This non-evolutionary component is intended to improve comprehensibility in the final hierarchy.

The only method of optimization currently being considered is term modification or elimination by examining restrictions. The idea is to check all of the terms, exclude the specified ranges, and see if there is only one possible value left. 


\section{Examples}

a) $(\mathrm{A}>0)^{\wedge}(\mathrm{A}<2)$, where $\mathrm{A}$ is an integer value, can be replaced by $(\mathrm{A}=1)$

b) $(\mathrm{A}>=2)^{\wedge}(\mathrm{A}<3)$, where $\mathrm{A}$ is an integer value, can be replaced by $(\mathrm{A}=2)$

c) $(\mathrm{A}>=2)^{\wedge}(\mathrm{A}<=2)$, where $\mathrm{A}$ is an integer value, can be replaced by $(\mathrm{A}=2)$

d) $(\mathrm{A} !=\mathrm{X})^{\wedge}(\mathrm{A} !=\mathrm{Y})$ in a set of $\{\mathrm{X}, \mathrm{Y}, \mathrm{Z}\}$ or integers with range 3 , then $(\mathrm{A}=\mathrm{Z})$

Depending on the parameters being used for a particular experiment, this optimization technique might never be used. In particular the case where a bonus is awarded for parsimony, these rules might have already been optimized during the search. Because the search process is probabilistic, however, it might still make sense to perform optimization techniques on the rules in the hierarchy after the search is complete. 


\section{Appendix A: Hill Climbing example}

This appendix describes the results of using the Hill Climbing local search technique on a specific rule. The original reference to this example is in Chapter 5.11.4.3 Hill Climbing as a Rule Mutation Operator, starting on page 151.

The rule that the Hill Climbing procedure is being done on is the following:

If $(\mathrm{C} 1>\mathrm{C} 3) \wedge(\mathrm{C} 2<5) \wedge(\$ \mathrm{~B}=1)$ then $(\mathrm{CLASS}=1)$

Population Gen-Functions and Meta-Variables present at time of Hill Climbing

$\mathrm{GF}: \$ \mathrm{~A}[\mathrm{RGC}($ class $=$ Rank $)]$

GF: $\$ B[N E V($ class $=$ Rank $)=2\{$ Variable $\}]$

$\mathrm{MV}: \# \mathrm{C}[(\mathrm{C} 1>\mathrm{C} 3) \wedge(\mathrm{C} 5=\mathrm{C} 4)]$

$\mathrm{MV}: \# \mathrm{D}[(\mathrm{C} 1>\mathrm{C} 5) \wedge(\mathrm{S} 2 !=\mathrm{S} 4)]$

\section{$\underline{\text { Variables }}$}

$\mathrm{C} 1, \mathrm{C} 2, \mathrm{C} 3, \mathrm{C} 4, \mathrm{C} 5$ are all integer values in the range $[1, \ldots, 13]$.

Gen-Function $\$ \mathrm{~A}$ has a range of $[0, \ldots, 13]$.

Gen-Function $\$ B$ has a range of $[0, \ldots, 3]$.

\begin{tabular}{|c|c|l|}
\hline$\#$ & Step & Rule \\
\hline 1 & 1 & If $(\mathrm{C} 1>\mathrm{C} 3) \wedge(\mathrm{C} 2<5) \wedge(\$ \mathrm{~B}=1)$ then $(\mathrm{CLASS}=1)$ \\
\hline 2 & 2 & If $(\mathrm{C} 2<5) \wedge(\$ \mathrm{~B}=1)$ then $(\mathrm{CLASS}=1)$ \\
\hline 3 & 2 & If $(\mathrm{C} 1>\mathrm{C} 3) \wedge(\$ \mathrm{~B}=1)$ then $(\mathrm{CLASS}=1)$ \\
\hline 4 & 2 & If $(\mathrm{C} 1>\mathrm{C} 3) \wedge(\mathrm{C} 2<5)$ then $(\mathrm{CLASS}=1)$ \\
\hline 5 & 3 & If $(\mathrm{C} 2>\mathrm{C} 3) \wedge(\mathrm{C} 2<5) \wedge(\$ \mathrm{~B}=1)$ then $(\mathrm{CLASS}=1)$ \\
\hline 6 & 3 & If $(\mathrm{C} 4>\mathrm{C} 3) \wedge(\mathrm{C} 2<5) \wedge(\$ \mathrm{~B}=1)$ then $(\mathrm{CLASS}=1)$ \\
\hline 7 & 3 & If $(\mathrm{C} 5>\mathrm{C} 3) \wedge(\mathrm{C} 2<5) \wedge(\$ \mathrm{~B}=1)$ then $(\mathrm{CLASS}=1)$ \\
\hline
\end{tabular}




\begin{tabular}{|c|c|c|}
\hline 8 & 3 & If $(\mathrm{C} 1>\mathrm{C} 3) \wedge(\mathrm{C} 1<5) \wedge(\$ \mathrm{~B}=1)$ then $(\mathrm{CLASS}=1)$ \\
\hline 9 & 3 & If $(\mathrm{C} 1>\mathrm{C} 3) \wedge(\mathrm{C} 3<5) \wedge(\$ \mathrm{~B}=1)$ then $(\mathrm{CLASS}=1)$ \\
\hline 10 & 3 & If $(\mathrm{C} 1>\mathrm{C} 3) \wedge(\mathrm{C} 4<5) \wedge(\$ \mathrm{~B}=1)$ then $(\mathrm{CLASS}=1)$ \\
\hline 11 & 3 & If $(\mathrm{C} 1>\mathrm{C} 3) \wedge(\mathrm{C} 5<5) \wedge(\$ \mathrm{~B}=1)$ then $(\mathrm{CLASS}=1)$ \\
\hline 12 & 5 & If $(\mathrm{C} 2<5) \wedge(\$ \mathrm{~B}=1) \wedge(\# \mathrm{C})$ then $(\mathrm{CLASS}=1)$ \\
\hline 13 & 5 & If $(\mathrm{C} 1>\mathrm{C} 3) \wedge(\mathrm{C} 2<5) \wedge(\$ \mathrm{~B}=1) \wedge(\# \mathrm{D})$ then $(\mathrm{CLASS}=1)$ \\
\hline 14 & 6 & If $(\mathrm{C} 1=\mathrm{C} 3) \wedge(\mathrm{C} 2<5) \wedge(\$ \mathrm{~B}=1)$ then $(\mathrm{CLASS}=1)$ \\
\hline 15 & 6 & If $(\mathrm{C} 1 !=\mathrm{C} 3) \wedge(\mathrm{C} 2<5) \wedge(\$ \mathrm{~B}=1)$ then $(\mathrm{CLASS}=1)$ \\
\hline 16 & 6 & If $(\mathrm{C} 1<\mathrm{C} 3) \wedge(\mathrm{C} 2<5) \wedge(\$ \mathrm{~B}=1)$ then $(\mathrm{CLASS}=1)$ \\
\hline 17 & 6 & If $(\mathrm{C} 1>\mathrm{C} 3) \wedge(\mathrm{C} 2=5) \wedge(\$ \mathrm{~B}=1)$ then $(\mathrm{CLASS}=1)$ \\
\hline 18 & 6 & If $(\mathrm{C} 1>\mathrm{C} 3) \wedge(\mathrm{C} 2>5) \wedge(\$ \mathrm{~B}=1)$ then $(\mathrm{CLASS}=1)$ \\
\hline 19 & 6 & If $(C 1>C 3) \wedge(C 2 !=5) \wedge(\$ B=1)$ then $($ CLASS $=1)$ \\
\hline 20 & 4 & If $(\mathrm{C} 1>\mathrm{C} 3) \wedge(\mathrm{C} 2<\mathrm{C} 1) \wedge(\$ \mathrm{~B}=1)$ then $(\mathrm{CLASS}=1)$ \\
\hline 21 & 4 & If $(\mathrm{C} 1>\mathrm{C} 3) \wedge(\mathrm{C} 2<\mathrm{C} 3) \wedge(\$ \mathrm{~B}=1)$ then $(\mathrm{CLASS}=1)$ \\
\hline 22 & 4 & If $(\mathrm{C} 1>\mathrm{C} 3) \wedge(\mathrm{C} 2<\mathrm{C} 4) \wedge(\$ \mathrm{~B}=1)$ then $(\mathrm{CLASS}=1)$ \\
\hline 23 & 4 & If $(\mathrm{C} 1>\mathrm{C} 3) \wedge(\mathrm{C} 2<\mathrm{C} 5) \wedge(\$ \mathrm{~B}=1)$ then $(\mathrm{CLASS}=1)$ \\
\hline 24 & 7 & If $(\mathrm{C} 1>\mathrm{C} 3) \wedge(\mathrm{C} 2<4) \wedge(\$ \mathrm{~B}=1)$ then $(\mathrm{CLASS}=1)$ \\
\hline 25 & 7 & If $(\mathrm{C} 1>\mathrm{C} 3) \wedge(\mathrm{C} 2<3) \wedge(\$ \mathrm{~B}=1)$ then $(\mathrm{CLASS}=1)$ \\
\hline 26 & 7 & If $(\mathrm{C} 1>\mathrm{C} 3) \wedge(\mathrm{C} 2<2) \wedge(\$ \mathrm{~B}=1)$ then $(\mathrm{CLASS}=1)$ \\
\hline 27 & 7 & If $(\mathrm{C} 1>\mathrm{C} 3) \wedge(\mathrm{C} 2<1) \wedge(\$ \mathrm{~B}=1)$ then $(\mathrm{CLASS}=1)$ \\
\hline 28 & 7 & If $(\mathrm{C} 1>\mathrm{C} 3) \wedge(\mathrm{C} 2<6) \wedge(\$ \mathrm{~B}=1)$ then $(\mathrm{CLASS}=1)$ \\
\hline 29 & 7 & If $(\mathrm{C} 1>\mathrm{C} 3) \wedge(\mathrm{C} 2<7) \wedge(\$ \mathrm{~B}=1)$ then $(\mathrm{CLASS}=1)$ \\
\hline 30 & 7 & If $(\mathrm{C} 1>\mathrm{C} 3) \wedge(\mathrm{C} 2<8) \wedge(\$ \mathrm{~B}=1)$ then $(\mathrm{CLASS}=1)$ \\
\hline 31 & 7 & If $(\mathrm{C} 1>\mathrm{C} 3) \wedge(\mathrm{C} 2<9) \wedge(\$ \mathrm{~B}=1)$ then $(\mathrm{CLASS}=1)$ \\
\hline 32 & 7 & If $(\mathrm{C} 1>\mathrm{C} 3) \wedge(\mathrm{C} 2<11) \wedge(\$ \mathrm{~B}=1)$ then $(\mathrm{CLASS}=1)$ \\
\hline 33 & 8 & If $(\mathrm{C} 1>\mathrm{C} 3) \wedge(\mathrm{C} 2<5) \wedge(\$ \mathrm{~B}=0)$ then $(\mathrm{CLASS}=1)$ \\
\hline 34 & 8 & If $(\mathrm{C} 1>\mathrm{C} 3) \wedge(\mathrm{C} 2<5) \wedge(\$ \mathrm{~B}=2)$ then $(\mathrm{CLASS}=1)$ \\
\hline 35 & 8 & If $(\mathrm{C} 1>\mathrm{C} 3) \wedge(\mathrm{C} 2<5) \wedge(\$ \mathrm{~B}=3)$ then $(\mathrm{CLASS}=1)$ \\
\hline 36 & 9 & If $(\mathrm{Cl}>\mathrm{C} 3) \wedge(\mathrm{C} 2<5) \wedge(\$ \mathrm{~B}=1) \wedge(\$ \mathrm{~A}=0)$ then $(\mathrm{CLASS}=1)$ \\
\hline 37 & 9 & If $(\mathrm{C} 1>\mathrm{C} 3) \wedge(\mathrm{C} 2<5) \wedge(\$ \mathrm{~B}=1) \wedge(\$ \mathrm{~A}=1)$ then $(\mathrm{CLASS}=1)$ \\
\hline 38 & 9 & If $(\mathrm{Cl}>\mathrm{C} 3) \wedge(\mathrm{C} 2<5) \wedge(\$ \mathrm{~B}=1) \wedge(\$ \mathrm{~A}=2)$ then $(\mathrm{CLASS}=1)$ \\
\hline 39 & 9 & If $(\mathrm{Cl}>\mathrm{C} 3) \wedge(\mathrm{C} 2<5) \wedge(\$ \mathrm{~B}=1) \wedge(\$ \mathrm{~A}=3)$ then $(\mathrm{CLASS}=1)$ \\
\hline 40 & 9 & If $(\mathrm{C} 1>\mathrm{C} 3) \wedge(\mathrm{C} 2<5) \wedge(\$ \mathrm{~B}=1) \wedge(\$ \mathrm{~A}=4)$ then $(\mathrm{CLASS}=1)$ \\
\hline 41 & 9 & If $(\mathrm{C} 1>\mathrm{C} 3) \wedge(\mathrm{C} 2<5) \wedge(\$ \mathrm{~B}=1) \wedge(\$ \mathrm{~A}=5)$ then $(\mathrm{CLASS}=1)$ \\
\hline 42 & 9 & If $(\mathrm{C} 1>\mathrm{C} 3) \wedge(\mathrm{C} 2<5) \wedge(\$ \mathrm{~B}=1) \wedge(\$ \mathrm{~A}=6)$ then $(\mathrm{CLASS}=1)$ \\
\hline 43 & 9 & If $(\mathrm{C} 1>\mathrm{C} 3) \wedge(\mathrm{C} 2<5) \wedge(\$ \mathrm{~B}=1) \wedge(\$ \mathrm{~A}=7)$ then $(\mathrm{CLASS}=1)$ \\
\hline 44 & 9 & If $(\mathrm{C} 1>\mathrm{C} 3) \wedge(\mathrm{C} 2<5) \wedge(\$ \mathrm{~B}=1) \wedge(\$ \mathrm{~A}=8)$ then $(\mathrm{CLASS}=1)$ \\
\hline 45 & 9 & If $(\mathrm{C} 1>\mathrm{C} 3) \wedge(\mathrm{C} 2<5) \wedge(\$ \mathrm{~B}=1) \wedge(\$ \mathrm{~A}=9)$ then $(\mathrm{CLASS}=1)$ \\
\hline 46 & 9 & If $(\mathrm{C} 1>\mathrm{C} 3) \wedge(\mathrm{C} 2<5) \wedge(\$ \mathrm{~B}=1) \wedge(\$ \mathrm{~A}=10)$ then $(\mathrm{CLASS}=1)$ \\
\hline 47 & 9 & If $(\mathrm{C} 1>\mathrm{C} 3) \wedge(\mathrm{C} 2<5) \wedge(\$ \mathrm{~B}=1) \wedge(\$ \mathrm{~A}=11)$ then $(\mathrm{CLASS}=1)$ \\
\hline 48 & 9 & If $(\mathrm{C} 1>\mathrm{C} 3) \wedge(\mathrm{C} 2<5) \wedge(\$ \mathrm{~B}=1) \wedge(\$ \mathrm{~A}=12)$ then $(\mathrm{CLASS}=1)$ \\
\hline 49 & 9 & If $(\mathrm{C} 1>\mathrm{C} 3) \wedge(\mathrm{C} 2<5) \wedge(\$ \mathrm{~B}=1) \wedge(\$ \mathrm{~A}=13)$ then $(\mathrm{CLASS}=1)$ \\
\hline
\end{tabular}




\section{Appendix B: Gen-Set operations and properties}

A Gen-Set is a basic type in EvRFind that is used to represent a set of numbers, and can be used in the place of a standard constant value. This type has a special use within EvRFind, and because of this it does not follow all of the standard mathematical rules that normally apply to sets.

\section{Gen-Set Operations}

\section{Equality}

In this case, the values contained within the set are disjunctive.

$A=\{2,4,7\}$ is true if the value represented by $A$ is 2,4 , or 7 . When two sets are compared for equality then it will be true if the sets are semantically identical. A subset of values in one set is not equal to a second set.

\section{Inequality}

In this case, the values contained within the set are conjunctive.

$\mathrm{A} !=\{2,4,7\}$ is true if the value represented by $\mathrm{A}$ is not equal to any of the values in the set. Specifically, this will evaluate to true if A does not represent 2,4 , or 7 . When two sets are compared then there must be at least one less or one differing element between the sets in order for the result to be true. 


\section{Greater than}

In this case, the values in the set are conjunctive. $A>\{2,4,7\}$ is true if $A$ is greater than the highest value in the set, 7 . When two sets are compared, the result evaluates to true if all of the values in the first set are greater than the maximum value in the second set.

\section{Greater than or equal to}

$A>=\{2,4,7\}$ is true if $A$ is equal to one of the elements in the set $(2,4$, or 7$)$, or if $A$ is greater than the highest value in the set, 7 . When two sets are compared this does not behave in the same way that a typical "greater than or equal to" comparison does. It is more accurately described as a "greater than and contains" because it evaluates to true if the maximum element in the first set is greater than the maximum element in the second set, and the two sets have at least one element in common.

\section{Less than}

In this case, the values in the set are conjunctive.

$\mathrm{A}<\{2,4,7\}$ is true if $\mathrm{A}$ is greater less than the lowest value in the set, 2 . When two sets are compared, the result evaluates to true if all of the values in the first set are less than the minimum value in the second set.

\section{Less than or equal to}

$\mathrm{A}<=\{2,4,7\}$ is true if $\mathrm{A}$ is equal to one of the elements in the set $(2,4$, or 7$)$, or if $\mathrm{A}$ is less than the lowest value in the set, 2. As in the odd case of "greater than or equal to", this is more appropriately describes as "less than and contains". The comparison will 
evaluate to true if the minimum element in the first set is less than the minimum value in the second set, and there is at least one element in common between the two sets.

\section{Properties}

1) Violates anti-symmetry: $(\mathrm{A}<=\mathrm{B})$ and $(\mathrm{B}<=\mathrm{A})$ without $\mathrm{A}$ being identical to $\mathrm{B}$

2) For the equations: $\{2,4,6\}[<=,>=, !=]\{3,4,5\}$
a. Transitivity is valid for $\{<,>\}$, but not for $\{<=,>=\}$
b. Reflexivity valid for $\{<=,>=\}$, but not for $\{<,>\}$
c. Non-total: $<=,>=,<,>$

3) A non-empty set is greater than the empty set.

4) A non-empty set is always greater than or equal to the empty set.

5) An empty set is less than any non-empty set.

6) The empty set is a subset of every set, therefore it is always less than or equal to another set.

\section{Examples}
$\{1,3,5\}>=\{2,3,4\}$
The first set is greater than or equal to the second set
$\{1,2,3\}<=\{2,3,4\}$
It is also less than or equal to the second set
$\{5,6,7\}>\{1,2,4\}$
The first set is strictly greater than the second set
$\{1,2,5\} !=\{4,6,7\}$
These two sets are only "not equal" to each other 


\section{Appendix C: Definition and Scoring of Poker Hands}

Poker uses a standard deck of 52 unique playing cards. The ranking of the individual cards, from high to low, is Ace, King, Queen, Jack, 10, 9, 8, 7, 6, 5, 3, 2. There is no ranking between the suits. For example, the King of Hearts and King of Spades are of equal value in poker.

A poker hand consists of five cards. The categories of hand, from highest to lowest, are listed below. Any hand in a higher category beats any hand in a lower category. This means, for example, that any Three of a Kind hand beats any hand of Two Pairs. Between hands in the same category the rank of the individual cards decides which is better. This is more completely described below.

In games where a player has more than five cards and selects five to form a poker hand, the remaining cards do not play any part in the ranking. Poker ranks are always based on five cards only.

Following is a description of each possible hand. For information on the probability of drawing each hand, please refer to Appendix D: Calculation of Poker Hand Statistics, starting on page 305 . 


\section{C.1 Royal Flush}

This is the highest possible poker hand. It consists of an \{ace, king, queen, jack, and ten\}, all in the same suit. As all suits are equal, all royal flushes are considered equal.

\section{C.2 Straight Flush}

This hand consists of five cards of the same suit in sequence, such as fjack of clubs, ten of clubs, nine of clubs, eight of clubs, and seven of clubs\}. Between two straight flushes, the one containing the higher top card is a higher hand. An ace can be counted as low, such as in the case of $\{$ five, four, three, two, ace (all of hearts) $\}$, but its top card is the five, not the ace. This is the lowest type of straight flush. The cards cannot use the ace as a point in the middle. For example, \{four, three, two, ace, king (all of diamonds)\}, is not a valid straight flush.

\section{3 Four of a kind}

This hand consists of four cards of the same rank, such as four queens. The fifth card can be any other card. This combination is sometimes known as "quads". Between two four of a kind hands, the one with the higher set of four cards is better. For example, a hand of four jacks is beaten by a hand of four kings.

\section{4 Full House}

This hand consists of three cards of one rank and two cards of another rank. An example of this is three sevens and two tens. When comparing full houses, the rank of the three 
cards determines which is higher. For example, a hand made up of three nines and two fours beats a hand made up of three eights and two aces.

\section{5 Flush}

This hand consists of five cards of the same suit, excluding the cases where the hand is a royal flush or a straight flush. When comparing two flushes, the highest card determines which hand is higher. If the highest cards are equal then the second highest card is compared; if those are equal too, then the third highest card, and so on. For example, a hand made up of \{queen, jack, eight, three, and two (all of spades) \} beats a hand made up of \{queen, jack, seven, six, four (all of hearts)\} because the eight in the first hand is higher than the seven in the second hand.

\section{6 Straight}

This hand consists of five cards, of mixed suits, in sequence according to their rank. For example, \{queen, jack, ten, nine, eight - where at least one card is of a different suit than the others $\}$, is a straight. When comparing two straights, the one with the higher ranking top card is better. Ace can count high or low in a straight, but as in the case of a straight flush, the ace can only act as an end point.

\section{C.7 Three of a Kind}

This hand consists of three cards of the same rank, plus two other cards. The two other cards must be of different ranks than the first three cards, and also different from each 
other. This combination is also known as "Triplets" or "Trips". When comparing two triplets, the hand that contains the three cards of higher rank is the better hand. For example; $\{6,6,6,3,2\}$ beats a hand made up of $\{4,4,4$, king, jack $\}$.

\section{C.8 Two Pairs}

This hand consists of two pairs of cards of equal rank. The first pair of cards must be of the same rank, and the second pair must also have the same rank as each other, but not the same as the original two. The remaining card must be a different rank than the initial four cards. When comparing hands with two pairs, the hand with the highest pair wins, irrespective of the rank of the other cards. For example, \{jack, jack, 2, 2, 4\} beats a hand made up of $\{10,10,9,9$, ace $\}$ because the jacks beat the tens. If the higher pairs are equal then the lower pairs are compared. Finally, if both pairs are the same then the odd cards are compared.

\section{C.9 Pair}

This hand consists of two cards with the same rank, and three other cards that do not have the same rank as the first two, or the same rank as each other. When comparing two such hands, the one with the higher pair is better. For example, $\{6,6,4,3,2\}$ is a better hand than $\{5,5$, ace, king, queen $\}$. If the pairs are equal then the highest ranking odd cards are compared. If these are also equal, then the second highest, and if necessary, the third highest ranking cards are compared. 


\section{C.10 High Card}

This is the name given to a hand that consists of five cards and does not form any of the combinations listed above. When comparing two such hands, the one with the better highest ranking card wins. If the highest cards are equal then the second highest cards are compared, and so on. For example, a hand made up of \{ace, jack, 6, 5, 3\} beats a hand made up of $\{$ ace, jack, $6,5,2\}$ because the three in the first hand is better than the two in the second hand. 


\section{Appendix D: Calculation of Poker Hand Statistics}

Following is the calculation for probability of each possible hand in poker. For a detailed description on the various hands, please refer to Appendix C: Definition and Scoring of Poker Hands, page 300.

\section{D.1 Total hands}

52 ways to deal the first card, 51 ways to deal the second card, 50 ways to deal the third card, 49 ways to deal the fourth card, 48 ways to deal the fifth card.

$52 \times 51 \times 50 \times 49 \times 48=311875200$ total hands

\section{D.2 Royal flush}

There are 20 possible 10-Jack-Queen-King-Ace cards, of any suit, for the first card. Beyond the first card, the suits must be the same and the ranks have to be in sequential order.

$20 \times 4 \times 3 \times 2 \times 1=480$ total royal flush hands

\section{D.3 Straight flush}

The first card must be Ace-2-3-4-5-6-7-8-9. If the first card is allowed to be 10 then the royal flush hands will be included. There are 36 possibilities for the first card, and beyond the first card the suits must be the same and the ranks in sequential order. $36 \times(5 \times 4 \times 3 \times 2$ for the positions $)=4320$ total straight flush hands 


\section{4 Four of a kind}

The first card can be any choice. Three of the remaining four cards must be of the same rank, and the last choice is any remaining card in the deck.

$52 \times 48 \times 5 \times(3 \times 2 \times 1$ for the positions $)=74880$ total four of a kind hands

\section{D.5 Full house}

The first card can be any choice. One of the remaining four cards must be of the same rank. The three cards left must have the same rank as each other, but different from the first two.

$52 \times 48 \times 10 \times(3 \times 2 \times 3$ for the positions $)=449280$ total full house hands

\section{D.6 Flush}

The first card can be any choice. The next four cards must be of the same suit as the first card.

$52 \times 12 \times 11 \times 10 \times 9=617760$ total flush hands, including the straight flush and royal flush hands. Subtracting these two subsets leaves a total of $612960 \mathrm{flush}$ hands.

\section{D.7 Straight}

As with the straight flush, there are 40 possible first cards. Each of the remaining cards have four choices (rank is fixed, but the suit is not). $40 \times 4 \times 4 \times 4 \times 4 \times(5 \times 4 \times 3 \times 2$ for the positions $)=1228800$ straight hands, including all of the straight flush and royal flush hands. Subtracting these two subsets leaves a total of 1224000 straight hands. 


\section{D.8 Three of a kind}

The first card can be any choice. Two of the remaining cards have to be of the same rank as the first, and neither the final two cards can be of the same rank as the first, or the same rank as each other.

$52 \times 48 \times 44 \times 10 \times(3 \times 2$ for positions $)=6589440$ total three of $a$ kind hands

\section{D.9 Two pairs}

The first card can be any choice. One of the remaining cards must be of the same rank. Two of the three cards left must be of the same rank as each other, but not match the first card. The final card must not be the same rank as any others.

$52 \times 48 \times 44 \times 15 \times(3 \times 3$ for positions $)=14826240$ total two pairs hands

\section{D.10 One pair}

The first card can be any choice. Of the remaining cards, exactly one must match the rank of the first, and all others must have ranks that are distinct from each other as well as different from the first card.

$52 \times 48 \times 44 \times 40 \times(10 \times 3$ for positions $)=131788800$ total one pair hands

\section{D.11 Nothing (High Card or Bust)}

This value is the total number of hands with all of the others subtracted.

156304800 total hands are classified as nothing. 


\section{Appendix E: Video Poker Scoring System}

In addition to the simple scoring system that was described in Chapter 6: The Poker

Hand Dataset, starting on page 167 , the following table also describes the Video Poker scoring system:

\begin{tabular}{|l|c|l|l|l|}
\hline S1 & S2 & \multicolumn{1}{|c|}{ Name } & \multicolumn{1}{|c|}{ Description } & \multicolumn{1}{|c|}{ Example } \\
\hline 16 & 9 & Royal Flush (RF) & Ace $\rightarrow$ Ten of same suit & AH KH QH JH 10H \\
\hline 15 & 8 & Straight Flush & Five sequential ranks, same suit & 4C 5C 6C 7C 8C \\
\hline 14 & 7 & Four of a kind & Four of the same rank & 2H 2D 2S 2C 8S \\
\hline 13 & 6 & Full house & Three of a kind plus one pair & 3D 3C 7H 7S 7D \\
\hline 12 & 5 & Flush & Five cards of the same suit & 2C 3C 6C 9C AC \\
\hline 11 & 4 & Straight & Five sequential ranks & 3C 4C 5D 6H 7D \\
\hline 10 & 0,1 & Four to RF & Four cards towards Royal Flush & AD KD QD JD 4S \\
\hline 9 & 3 & Three of a kind & Three equal ranks & 5H 5S 5D 3C 7H \\
\hline 8 & 2 & Two pairs & Two pairs of equal ranks & 4H 4S 9D 9S 7C \\
\hline 7 & 1 & One high pair & Two equal ranks (Jacks or better) & QH QD 2C KH 9S \\
\hline 6 & $0-1$ & Four to Flush & Four cards of the same suit & 2C 5C 7C 9C 4H \\
\hline 5 & $0-1$ & Four to Straight & Four sequential cards (2 r $<A)$ & 3H 4D 5D 6C AS \\
\hline 4 & $0-3$ & Three to RF & Three cards towards Royal Flush & KD QD JD 9S 8H \\
\hline 3 & 1 & One low pair & Two equal ranks (Ten or less) & 5H 5C 7D JS 2D \\
\hline 2 & 0 & Two high cards & Two cards (Jacks or better) & JD QC 2H 9C 5D \\
\hline 1 & 0 & One high card & One card (Jack or better) & AS 4H 8D 3S 10H \\
\hline 0 & 0 & Nothing & No useful cards in the group & 2S 3D 6H 9C 10H \\
\hline
\end{tabular}

Table 8.1: Comparing Video Poker and Simple Poker scoring systems

The S1 (score 1) column denotes the score that would be calculated if using the Video poker ranking system, while the $\mathbf{S} 2$ (score 2) column contains scores that simply rank the ten different possible poker hands.

The main difference between these two systems is that video poker hands are not necessarily final. At the option of the player, one or more of the cards can be discarded and replaced with random cards from the same deck. This can occur only one time, but it means that most hands have the potential to be improved upon. This additional step makes it necessary for the scoring system to recognize hands that have a potential value. 
A good example of a hand with potential is the "Four to Royal Flush", with a score of 10. In the simple scoring system this hand will have a score of either 0 or 1 , depending on whether a pair exists within the five cards. In the video poker scoring system, this has the potential to be transformed into a royal flush, a straight flush (low card 9, high card king), a non-flush straight, a non-straight flush, a pair, or nothing, depending on which card replaces the discard.

A second point concerning the video poker scoring system is the meaning of pairs. The breakdown of pairs based on value is only important for the video poker scoring system, where a single pair of rank ten or less is considered a losing hand. A single pair of Jacks, Queens, Kings, or Aces is considered a winning hand.

\begin{tabular}{|l|l|l|l|}
\hline Poker Hand & \# of hands & Probability & \# of combinations \\
\hline One pair $>=$ Jack & 337920 & 0.13002124 & 40550400 \\
\hline One pair $<=$ Ten & 760320 & 0.29254779 & 91238400 \\
\hline Total & $\mathbf{1 0 9 8 2 4 0}$ & $\mathbf{0 . 4 2 2 5 6 9 0 3}$ & $\mathbf{1 3 1 7 8 8 8 0 0}$ \\
\hline
\end{tabular}

Table 8.2: Breakdown of pair hands

Because of the extra classes, discovering rules that accurately classify hands using this scoring system is considerably more difficult. Consequently, experiments of this type were not done for this research. 


\section{Appendix F: Examples of minimal rule sets}

This appendix contains three examples of minimal rule sets that are required when generalization functions are not available, although relationships between attributes can still be expressed. Each of these sets of rules covers $100 \%$ the specified class from the Poker Hand dataset with $100 \%$ confidence. The three classes are: One pair, full house, and royal flush. References are also specified for the classes of two pairs, and four of a kind.

One pair

\section{Terms in the antecedent}

$(\mathrm{Cl}=\mathrm{C} 2) \wedge(\mathrm{Cl} !=\mathrm{C} 3) \wedge(\mathrm{C} 1 !=\mathrm{C} 4) \wedge(\mathrm{C} 1 !=\mathrm{C} 5) \wedge(\mathrm{C} 3 !=\mathrm{C} 4) \wedge(\mathrm{C} 3 !=\mathrm{C} 5) \wedge(\mathrm{C} 4 !=\mathrm{C} 5)$ $(\mathrm{Cl}=\mathrm{C} 3) \wedge(\mathrm{Cl} !=\mathrm{C} 2) \wedge(\mathrm{Cl} !=\mathrm{C} 4) \wedge(\mathrm{C} 1 !=\mathrm{C} 5) \wedge(\mathrm{C} 2 !=\mathrm{C} 4) \wedge(\mathrm{C} 2 !=\mathrm{C} 5) \wedge(\mathrm{C} 4 !=\mathrm{C} 5)$ $(\mathrm{C} 1=\mathrm{C} 4) \wedge(\mathrm{Cl} !=\mathrm{C} 2) \wedge(\mathrm{Cl} !=\mathrm{C} 3) \wedge(\mathrm{Cl} !=\mathrm{C} 5) \wedge(\mathrm{C} 2 !=\mathrm{C} 3) \wedge(\mathrm{C} 2 !=\mathrm{C} 5) \wedge(\mathrm{C} 3 !=\mathrm{C} 5)$ $(\mathrm{Cl}=\mathrm{C} 5) \wedge(\mathrm{C} 1 !=\mathrm{C} 2) \wedge(\mathrm{Cl} !=\mathrm{C} 3) \wedge(\mathrm{C} 1 !=\mathrm{C} 4) \wedge(\mathrm{C} 2 !=\mathrm{C} 3) \wedge(\mathrm{C} 2 !=\mathrm{C} 4) \wedge(\mathrm{C} 3 !=\mathrm{C} 4)$ $(\mathrm{C} 2=\mathrm{C} 3) \wedge(\mathrm{C} 1 !=\mathrm{C} 2) \wedge(\mathrm{C} 1 !=\mathrm{C} 4) \wedge(\mathrm{C} 1 !=\mathrm{C} 5) \wedge(\mathrm{C} 2 !=\mathrm{C} 4) \wedge(\mathrm{C} 2 !=\mathrm{C} 5) \wedge(\mathrm{C} 4 !=\mathrm{C} 5)$ $(\mathrm{C} 2=\mathrm{C} 4) \wedge(\mathrm{C} 1 !=\mathrm{C} 2) \wedge(\mathrm{C} 1 !=\mathrm{C} 3) \wedge(\mathrm{C} 1 !=\mathrm{C} 5) \wedge(\mathrm{C} 2 !=\mathrm{C} 3) \wedge(\mathrm{C} 2 !=\mathrm{C} 5) \wedge(\mathrm{C} 3 !=\mathrm{C} 5)$ $(\mathrm{C} 2=\mathrm{C} 5) \wedge(\mathrm{C} 1 !=\mathrm{C} 2) \wedge(\mathrm{C} 1 !=\mathrm{C} 3) \wedge(\mathrm{C} 1 !=\mathrm{C} 4) \wedge(\mathrm{C} 2 !=\mathrm{C} 3) \wedge(\mathrm{C} 2 !=\mathrm{C} 4) \wedge(\mathrm{C} 3 !=\mathrm{C} 4)$ $(\mathrm{C} 3=\mathrm{C} 4) \wedge(\mathrm{C} 1 !=\mathrm{C} 2) \wedge(\mathrm{C} 1 !=\mathrm{C} 3) \wedge(\mathrm{C} 1 !=\mathrm{C} 5) \wedge(\mathrm{C} 2 !=\mathrm{C} 3) \wedge(\mathrm{C} 2 !=\mathrm{C} 5) \wedge(\mathrm{C} 3 !=\mathrm{C} 5)$ $(\mathrm{C} 3=\mathrm{C} 5) \wedge(\mathrm{C} 1 !=\mathrm{C} 2) \wedge(\mathrm{C} 1 !=\mathrm{C} 3) \wedge(\mathrm{Cl} !=\mathrm{C} 4) \wedge(\mathrm{C} 2 !=\mathrm{C} 3) \wedge(\mathrm{C} 2 !=\mathrm{C} 4) \wedge(\mathrm{C} 3 !=\mathrm{C} 4)$ $(\mathrm{C} 4=\mathrm{C} 5) \wedge(\mathrm{C} 1 !=\mathrm{C} 2) \wedge(\mathrm{C} 1 !=\mathrm{C} 3) \wedge(\mathrm{C} 1 !=\mathrm{C} 4) \wedge(\mathrm{C} 2 !=\mathrm{C} 3) \wedge(\mathrm{C} 2 !=\mathrm{C} 4) \wedge(\mathrm{C} 3 !=\mathrm{C} 4)$

As can be seen in the table above, a minimum of 10 rules are required in order to cover the one pair class from the poker hand dataset. 


\section{Full House}

\begin{tabular}{|l|}
\hline Terms in the antecedent \\
\hline$(\mathrm{C} 1=\mathrm{C} 2) \wedge(\mathrm{C} 3=\mathrm{C} 4) \wedge(\mathrm{C} 4=\mathrm{C} 5)$ \\
\hline$(\mathrm{C} 1=\mathrm{C} 3) \wedge(\mathrm{C} 2=\mathrm{C} 4) \wedge(\mathrm{C} 4=\mathrm{C} 5)$ \\
\hline$(\mathrm{C} 1=\mathrm{C} 5) \wedge(\mathrm{C} 2=\mathrm{C} 3) \wedge(\mathrm{C} 3=\mathrm{C} 4)$ \\
\hline$(\mathrm{C} 2=\mathrm{C} 3) \wedge(\mathrm{Cl}=\mathrm{C} 4) \wedge(\mathrm{C} 4=\mathrm{C} 5)$ \\
\hline$(\mathrm{C} 2=\mathrm{C} 4) \wedge(\mathrm{Cl}=\mathrm{C} 3) \wedge(\mathrm{C} 3=\mathrm{C} 5)$ \\
\hline $\mathrm{C} 2=\mathrm{C} 5) \wedge(\mathrm{C} 1=\mathrm{C} 3) \wedge(\mathrm{C} 3=\mathrm{C} 4)$ \\
\hline $\mathrm{C} 3=\mathrm{C} 4) \wedge(\mathrm{Cl}=\mathrm{C} 2) \wedge(\mathrm{C} 2=\mathrm{C} 5)$ \\
\hline$(\mathrm{C} 3=\mathrm{C} 5) \wedge(\mathrm{Cl}=\mathrm{C} 2) \wedge(\mathrm{C} 2=\mathrm{C} 4)$ \\
\hline $\mathrm{C} 4=\mathrm{C} 5) \wedge(\mathrm{Cl}=\mathrm{C} 2) \wedge(\mathrm{C} 2=\mathrm{C} 3)$ \\
\hline
\end{tabular}

With only nine rules required to fully classify the full house class, it is roughly of the same complexity as the one pair class.

\section{$\underline{\text { Royal Flush }}$}

The royal flush class is very specific and in order to cover $100 \%$ of the class with $100 \%$ confidence, it requires at least 120 rules without the generalization available when using a Gen-Function. The first component is checking to make sure that the suits all match:

If $(\mathrm{S} 1=\mathrm{S} 2) \wedge(\mathrm{S} 2=\mathrm{S} 3) \wedge(\mathrm{S} 3=\mathrm{S} 4) \wedge(\mathrm{S} 4=\mathrm{S} 5) \wedge \ldots$ then $(\mathrm{CLASS}=9)$

Missing from the above example rule is the remainder of the antecedent that follows specification of the card suits. The remaining terms deal with the values for the card ranks. Two examples, representing the first and last element in a sorted list, are as follows:

$$
\begin{aligned}
& \ldots \wedge(\mathrm{Cl}=1) \wedge(\mathrm{C} 2=10) \wedge(\mathrm{C} 3=11) \wedge(\mathrm{C} 4=12) \wedge(\mathrm{C} 5=13) \text { then }(\mathrm{CLASS}=9) \\
& . \\
& \ldots \wedge(\mathrm{Cl}=13) \wedge(\mathrm{C} 2=12) \wedge(\mathrm{C} 3=11) \wedge(\mathrm{C} 4=10) \wedge(\mathrm{C} 5=1) \text { then }(\mathrm{CLASS}=9)
\end{aligned}
$$




\section{$\underline{\text { Two Pairs }}$}

The list of rules that is required to classify two pairs is available in Chapter 0 : Initial experiments, starting on page 193 .

\section{Four of a Kind}

The list of the rules that is required to classify four of a kind is available in Chapter 0 : Concept of four of a kind, starting on page 210 . 


\section{Appendix G: Future Gen-Function Primitives}

Given the positive results that have been achieved using the limited number of Gen-

Functions that are implemented in the current version of EvRFind, a number of new

primitives are being planned. The tentative list is in the following table.

\begin{tabular}{|c|l|l|}
\hline Symbol & Parameters & Description \\
\hline CTS & $\begin{array}{l}\text { Attribute class, } \\
\text { value list }\end{array}$ & $\begin{array}{l}\text { The number of values that are contained within the } \\
\text { specified attribute class }\end{array}$ \\
\hline CVA & Attribute list & The number of consecutive values in the list \\
\hline CVC & Attribute class & The number of consecutive values within the class. \\
\hline DVA & Two attributes & Division of first value by the second value \\
\hline DVC & Attribute, constant & Division of first value by the specified constant value \\
\hline GCD & Attribute list & Greatest common divisor of the values \\
\hline LCM & Attribute list & Lowest common multiple of the values \\
\hline MDA & Two attributes & Modulus of the first value divided by the second \\
\hline MDC & Attribute, constant & Modulus of the first value divided by the constant \\
\hline NDA & $\begin{array}{l}\text { Attribute list, } \\
\text { constant }\end{array}$ & $\begin{array}{l}\text { Return the number of values in the attributes that } \\
\text { differ by at most the specified constant }\end{array}$ \\
\hline NDV & $\begin{array}{l}\text { Attribute class, } \\
\text { constant }\end{array}$ & $\begin{array}{l}\text { Return the number of values in the specified class } \\
\text { that differ by at most the specified constant }\end{array}$ \\
\hline PWR & Attribute, constant & Return: attribute to the power of constant \\
\hline RND & Attribute or GF & Round the attribute or function result down to integer \\
\hline RNU & Attribute or GF & Round the attribute or function result up to integer \\
\hline ROT & Attribute, constant & Return: attribute root constant \\
\hline SBA & Two attributes & Subtraction of the second attribute from the first \\
\hline SBC & Attribute, constant & Subtraction of the constant from the attribute \\
\hline
\end{tabular}




\section{Appendix H: Default Parameter Settings}

EvRFind has a number of options that are configurable for use during experiments, and not all of them are described in complete detail. Following is a list of options that represent the default configuration and can be used to reproduce the majority of experiments described in this research.

\section{$\underline{\text { Rule options }}$}

Maximum number of conjunctions in antecedent (first generation): 1

Maximum number of conjunctions in antecedent (beyond first generation): 8

Maximum number of conjunctions in consequent (first generation): 1

Maximum number of conjunctions in consequent (beyond first generation): 1

\section{Fitness options}

Encourage wider rule coverage (rule niching): True

Bonus for using variables: True

Bonus for using Meta-Variables: True

Bonus for using Gen-Functions: True

Reward shorter rules (parsimonious): True

Reward longer rules (overfit): False

Use protected fitness in hierarchy: (Varied - This is the HPF option that was evaluated)

Variable reward (dataset size): True

Static reward value: 0.1 [This was not used often - normally variable reward] 


\section{$\underline{\text { Termination }}$}

Number of generations: True, 50

Stop on full data coverage: True

Percentage of good rules: False, 80 [The 80 was not used]

Minimum fitness \%: 95 [This was not used]

\section{Evolutionary parameters}

Crossover rate: $80 \%$

Micro mutation rate: $0.06 \%$

Macro mutation rate: $0.01 \%$

\section{Custom confidence and support search options}

Confidence factor: $100 \%$ [For most experiments, otherwise specified]

Support factor: 100\% [For most experiments, otherwise specified]

Support factor adjustment: 1.0

Standard elitism: False

Deviation \%: 10 [This was not used]

Maximum \%: 10 [This was not used]

Classification elitism: True

\section{Intergenerational Processing}

Eliminate extra numeric restrictions: True

Eliminate conflicting restrictions: True 
Elimination removes more specific rules: False

Elimination removes more general rules: True

Use hill climbing between generations: True

Use tournament selection for hill climbing: True [In most cases]

Delete all zero-fitness rules during population trim: True

Perform full check for coverage between each generation: False

Recalculate hierarchy fitness after trimming rules: False

\section{Intergenerational rule abstraction and generalization options}

Create Meta-Variables: True

Automatic rule generalization: True

\section{Miscellaneous options}

Allow Boolean negation operator: False [This was not used - no Boolean variables]

Maintain discarded rule list to avoid duplicates: True

Initial random number seed: 0 [This typically spanned values 0 through 0 ]

Do not assume majority class: True

Assume majority class: False

Assign class based on distribution: False 


\section{References}

[Aha, 1991a] D.W. Aha, 1991a. Incremental constructive induction: an instance-based approached. Machine Learning: Proceedings of the Eighth International Workshop (ML91) (p. 117-121). San Maeo, CA: Morgan Kaufmann.

[Araujo, Lopes, Freitas, 1999] Dieferson L.A. Araujo, Heirot S. Lopes, Alex A Freitas, 1999. A Parallel Genetic Algorithm for Rule Discovery in Large Databases. Proceedings of 1999 IEEE Systems, Man and Cybernetics Conference. Vol III, p. 940-945. Tokyo, Japan.

[Araujo, Lopes, Freitas, 2000] Dieferson L.A. Araujo, Heitor S. Lopes, Alex A. Freitas, 2000. Rule Discovery with a Parallel Genetic Algorithm. In Workshop on Data Mining with Evolutionary Computation held in GECCO 2000, p. 89-92.

[Bagnall, Cawley, 2003] A.J. Bagnall, G.C. Cawley, 2003. Learning classifier systems for data mining: A comparison of XCS with other classifiers for the Forest Cover dataset. In Proceedings of the IEEE/INNS International Joint Conference on Neural Networks (IJCNN-2003), Volume 3, p. 1802-1807, Portland, Oregon, USA.

[Becker, Chambers, Wilks, 1998] Rick Becker, John Chambers, Allan Wilks. R: A Programming Environment for Data Analysis and Graphics. 
[Bensusan, Kuscu, 1996] H. Bensusan, I. Kuscu, 1996. Constructive Induction using Genetic Programming. In Evolutionary Computing and Machine Learning Workshop (ICML-96), Morgan Kaufmann.

[Berry, Linoff, 1997] Michael Berry, Gordon Linoff, 1997. Data Mining Techniques: For Marketing, Sales, and Customer Support. Wiley computer publishing.

[Blake, Murphy, 1998] C.L. Blake and P.M. Murphy, 1998. The UCI machine learning repository. <http://www.cs.uci.edu/mlearn/MLRepository.html>. In Irvine, CA: University of California, Department of Information and Computer Science.

[Bloedorn, Michalski, 1996] Eric Bloedorn, Ryszard S. Michalski, 1996. The AQ17DCI System for Data-Driven Constructive Induction and Its Application to the Analysis of World Economics. Proceedings of the International Symposium on Methodologies for Intelligent Systems, Zakopane, Poland.

[Breiman, Friedman, Ohlsen, Stone, 1984] L. Breiman, J. Friedman, R. Ohlsen, C. Stone, 1984. Classification and Regression Trees. Wadsworthand Brooks, Pacific Grove, CA.

[Burges, 1998] Christopher J. C. Burges, 1998. A tutorial on support vector machines for pattern recognition. Data Mining and Knowledge Discovery, Volume 2, p. 955-974. 
[Cattral, Oppacher, Deugo, 1999] R. Cattral, F. Oppacher, D. Deugo, 1999. Rule

Acquisition with a Genetic Algorithm. Proceedings of the 1999 Congress on

Evolutionary Computation, Washington, DC. IEEE Press.

[Cattral, Oppacher, Deugo, 2001] R. Cattral, F. Oppacher, D. Deugo, 2001. Supervised and unsupervised data mining with an evolutionary algorithm. The 2001 Congress on Evolutionary Computation (CEC01), p. 767-774. Seoul, Korea

[Chawla et al., 2002] Nitesh V. Chawla, Kevin W. Bowyer, Lawrence O. Hall, W. Philip Kegelmeyer. SMOTE: Synthetic Minority Over-sampling Technique. Journal of Artificial Intelligence and Research, volume 16, p. 321-357.

[Clark, Niblett, 1989] Peter Clark, Tim Nibleett. The CN2 induction algorithm. Machine Learning, volume 3, p. 261-284.

[Cordon, Herrera, 1999] Oscar Cordon, Francisco Herrera, 1999. A Two-Stage Evolutionary Process for Designing TSK Fuzzy Rule-Based Systems. IEEE Transactions on Systems, Man, and Cybernetics - Part B: Cybernetics, Vol. 29, No. 6.

[Cordon, Herrera, Villar, 2001] Oscar Cordon, Francisco Herrera, Pedro Villar, 2001. Generating the Knowledge Base of a Fuzzy rule-Based System by the Genetic Learning of the Data Base. IEEE Transactions on Fuzzy Ssytems, Vol. 9, No. 4. 
[Cortes, Vapnik, 1995] C. Cortes, V. Vapnik, 1995. Support vector networks. Machine Learning, volume 20 , p. 1-25.

[Cost, Salzberg, 1993] S. Cost, S. Salzberg, 1993. A Weighted Nearest Neighbour Algorithm for Learning with Symbolic Features. Machine Learning volume 10, p. 57-58.

[De Jong, Spears, 1991] Kenneth A. De Jong, William M. Spears, 1992. An Analysis of the Interacting Roles of Population Size and crossover in Genetic Algorithms. Parallel Problem Solving from Nature - Proceedings of $1^{\text {st }}$ Workshop.

[Drummond, Holte, 2003] Chris Drummond, Robert C. Holte, 2003. C4.5, Class Imbalance, and Cost Sensitivity: Why Under-Sampling beats Over-Sampling. ICML'2003 Workshop on Learning from Imbalanced Datasets II.

[Dryl et al., 2004] Jacek Dryl, Halina Kwasnicka, Jan Kornafel, Urszula markowskaKaczmar, Rafal Matkowski, Pawel Mikolajczyk, Jacek Tomasiak, 2004. Rule Discovery from Medical Data Using Genetic Algorithm. In proceedings of the Fourth International ICSC Symposium on Engineering of Intelligent Systems (EIS 2004).

[Duch et al., 1998] W. Duch, R. Adamczak, K. Grabczewski, G. Zal, 1998. A hybrid method for extraction of logical rules from data. Proceedings of Intelligent Information Systems VII, Malbork, Poland. 
[Duch, Grudzinski, 1999] Wlodzislaw Duch, Karol Grudzinski, 1999. Weighting and selction of features. In Proceedings of the Intelligent Information Systems VIII workshop.

[Duch, Adamczak, Grabczewski, 2000] Wlodzislaw Duch, Rafal Adamczak, Krzysztof Grabczewski, 2000. A new methodology of extraction, optimization and application of crisp and fuzzy logical rules. IEEE Trans Neural Networks, 11(2), p. 1-31.

[Freitas, 2002a] Alex A. Freitas, 2002. Data Mining and Knowledge Discovery With Evolutionary Algorithms. Springer Verlag, 2002. ISBN: 3-540-43331-7

[Freitas, 2002b] Alex A. Freitas, 2002. A survey of evolutionary algorithms for data mining and knowledge discovery. In: A. Ghosh and S. Tsutsui. (Eds.) Advances in Evolutionary Computation, p. 819-845. Springer-Verlag, 2002. ISBN: 3-540-43330-9

[Freund, Schapire, 1997] Y. Freund, R. E. Schapire, 1997. A decision-theoretic generalization of on-line learning and an application to boosting. Journal of Computer and System Sciences. 55(1), p. 119-139.

[Friedman et al., 1996] J. Friedman, R. Kohavi, Y. Yun, 1996. Lazy decision trees. In Proceedings of the Thirteenth National Conference on Artificial Intelligence, p. 717-724. Menlo Park, CA: The AAAI Press. 
[Friedman, Geiger, Goldszmidt, 1997] N. Friedman, D. Geiger, M. Goldszmidt, 1997. Bayesian network classifiers. Machine Learning 29, p. 131-163.

[Fröhlich, Chapelle, Schölkopf, 2003] Holger Fröhlich, Olivier Chapelle, Bernhard Schölkopf, 2003. Feature Selection for Support Vector Machines by Means of Genetic Algorithms. Proceedings of the $15^{\text {th }}$ IEEE International Conference on Tools with Artificial Intelligence, p. 142.

[Gao, Huang, Rong, 2005] Yang Gao, Joshua Zhexue Huang, Hongqiang Rong, 2005. Learning Classifier System Ensemble for Data Mining. In Proceedings of the Genetic and Evolutionary Computation Conference workshop program. ACM Press, Washington, D.C., USA, p. 63-66.

[Goldberg, 1989] David E. Goldberg, 1989. Genetic Algorithms in Search, Optimization, and Machine Learning. Addison-Wesley.

[Gopalan, Alhajj, Barker, 2006] Janaki Gopalan, Reda Alhajj, Ken Barker, 2006. Discovering Accurate and Interesting Classification Rules Using Genetic Algorithm. DMIN 2006, p. 289-395. CSREA Press.

[Guerra-Salcedo et al., 1999] César Guerra-Salcedo, Stephen Chen, Darrell Whitley, Stephen Smith, 1999. Fast and Accurate Feature Selection Using Hybrid Genetic Strategies. Proceedings of the 1999 Congress on Evolutionary Computation. 
[Gwee, Chang, 2003] Bah-Hwee Gwee, Joseph Sylvester Chang, 2003. A Hybrid Genetic Hill-climbing Algorithm for Four-coloring Map Problems. HIS 2003, p. 252-261.

[Hekanaho, 1998] J. Hekanaho, 1998. An Evolutionary Approach to Concept Learning, Ph.D Thesis, Department of Computer Science, Abo Akademi University.

[Holland, 1975] John H. Holland, 1975. Adaptation in Natural and Artificial Systems. University of Michigan Press (Ann Arbor).

[Holte, 1993] R.C. Holte, 1993. Very Simple Classification Rules Perform Well on Most Commonly Used Datasets. Machine Learning, volume 11, p. 63-90.

[Jourdan, Dhaenens, Talbi, 2001] Laetitia Jourdan, Clarisse Dhaenens, El-Ghazali Talbi, 2001. A Genetic Algorithm for Feature Selection in Data-Mining for Genetics. Metaheuristic International Conference 2001.

[Kim G, Kim S, 2000] Gyeonghwan Kim, Sekwang Kim, 2000. Feature Selection Using Genetic Algorithms for Handwritten Character Recognition. Proceedings of the Sixth ACM SIGKDD International Conference on Knowledge Discovery and Data Mining. 
[Kittler, 1986] J. Kittler, 1986. Feature selection and extraction. In T.Y. Young \& K. Fu (Eds.), Handbook of Pattern Recognition and Image Processing, p. 59-81. San Diego, CA: Academic Press.

[Kohavi, 1996] R. Kohavi, 1996. Scaling up the accuracy of naïve-Bayes classifiers: A decision-tree hybrid. In Proceedings of the Second International Conference on Knowledge Discovery and Data Mining, p. 202-207. Menlo Park, CA: The AAAI Press.

[Kokai, Toth, Zvada, 2002] G. Kokai, Z. Toth, S. Zvada, 2002. An Experimental Comparison of Genetic and Classical Concept Learning Methods. Proceedings of the Genetic and Evolutionary Computation Conference (p. 952). San Francisco, CA: Morgan Kaufmann.

[Kotecha, Gambhava, 2003] Ketan Kotecha, Nilesh Gambhava, 2003. A Hybrid Genetic Algorithm for Minimum Vertex Cover Problem. In Proceedings of the $1^{\text {st }}$ Indian International Conference on Artificial Intelligence. IICAI Press.

[Koza, 1992] John R. Koza, 1992. Genetic Programming: On the programming of computers by means of natural selection. MIT Press, Cambridge, Mass.

[Kubat, Matwin, 1997] M. Kubat, S. Matwin, 1997. Addressing the Curse of Imbalanced Training Sets: One Sided Selection. In Proceedings of the Fourteenth 
International Conference on Machine Learning, (Nashville, Tennessee), pp. 179-186, Morgan Kaufmann.

[Kusiak, Dixon, Shah, 2005] Andrew Kusiak, Bradley Dixon, Shital Shah, 2005. Predicting survival time for kidney dialysis patients: a data mining approach Computers in Biology and Medicine 35, p. 311-327.

[Langley, Iba, Thomas, 1992] P. Langley, W. Iba, K. Thomas, 1992. An analysis of Bayesian classifiers. In Proceedings of the Tenth National Conference of Artificial Intelligence. AAAI Press, p. 223-228.

[Lincoff, 1981] G. H. Lincoff, 1981. The Audubon Society Field Guide to North American Mushrooms. Alfred A. Knopf, New York.

[Matheus, Rendell, 1989] C.J. Matheus, L.A. Rendell, 1989. Constructive induction on decision trees. Proceedings of the Eleventh International Joint Conference on Artificial Intelligence (p. 645-650). Detroit, MI: Morgan Kaufmann.

[Michalski, Mozetic, Hong, Lavrac, 1986] R. Michalski, I. Mozetic, J. Hong, N. Lavrac, 1986. The AQ15 Inductive Learning System: an Overview and Experiments. In Proceedings of International Meeting on Advanced in Learning. 
[Minaei-Bidgoli, Punch, 2003] Behrouz Minaei-Bidgoli, William F. Punch, 2003. using Genetic Algorithms for Data Mining Optimization in an Educational Web-Based system. Proceedings of the Genetic and Evolutionary Computation Conference, p. 2252-2263.

[Morita et al., 2003] M. Morita, R. Sabourin, F. Bortolozzi, C. Suen, 2003. Unsupervised Feature Selection Using Multi-objective Genetic Algorithms for Handwritten Word Recognition. In Proceedings of the 7th International Conference on Document Analysis and Recognition (ICDAR).

[Moscato, 1989] Pablo Moscato, 1989. On Evolution, Search, Optimization, Genetic Algorithms and Martial Arts: Towards Memetic Algorithms. Technical Report number 790, Caltech Concurrent Computation Program, California Institute of Technology.

[Muggleton, 1991] S. Muggleton, 1991. Inductive Logic Programming, New Generations Computing, Volume 8, No. 4, p. 295-318.

[Neri, 1997] F. Neri, 1997. First Order Logic Concept Learning by means of a Distributed Genetic Algorithm. Ph.D. Thesis, Department of Computer Science, University of Torino, Italy.

[Newel, 1990] Allen Newel, 1990. Unified Theories of Cognition. Harvard University Press. 
[Otero, Silva, Freitas, Nievola, 2003] F.E.B. Otero, M.M.S. Silva, A.A. Freitas, J.C. Nievola, 2003. Genetic Programming for Attribute Construction in Data Mining. In proceedings of Genetic Programming: 6th European Conference (EuroGP-2003). Lecture Notes in Computer Science 2610, p. 284-393. Springer.

[Pazzani, 1996] M.J. Pazzani, 1996. Constructive induction of Cartesian product attributes. In Proceedings of the Tenth Conference on Uncertainty in Artificial Intelligence, p. 339-406. Seattle, WA: Morgan Kaufmann.

[Piramuthu, Sikora, 2006] Selwyn Piramuthu, Riyaz T. Sikora, 2006. Genetic Algorithm Based Learning Using Feature Construction. Artificial Intelligence and Data Mining Workshop in conjunction with the 2006 INFORMS Annual Meeting.

[Prügel-Bennett, 2004] Adam Prügel-Bennett, 2004. When A Genetic Algorithm Outperforms Hill-Climbing. Theoretical Computer Science Volume 320(1), p. 135-153. ISSN: 0304-3975.

[Quinlan, 1993] J.R. Quinlan, 1993. C4.5 is a licensed product that can be acquired from Morgan Kaufmann Publishers, Ann Arbor, Michigan.

[Quinlan, Cameron-Jones, 1993] J.R. Quinlan, R.M. Cameron-Jones, 1993. FOIL: A Midterm Report. In Proceedings of the Sixth European Conference on Machine Learning (ECML-93), Springer-Verlag, p. 3-20. 
[Quinlan, 1998] J.R. Quinlan, 1998. C5.0: An Informal Tutorial. RuleQuest.

$<$ http://www.rulequest.com/see5-win.html $>$

[Rish, 2001] Irina Rish, 2001. An empirical study of the naïve Bayes classifier. IJCAI 2001 Workshop on Empirical Methods in Artificial Intelligence.

$<$ http://www.research.ibm.com/people/r/rish/papers/RC22230.pdf>

[Rithoff et al., 2002] Oliver Ritthoff, Ralf Klinkenberg, Simon Fischer, Ingo Mierswa, 2002. A Hybrid Approach to Feature Selection and Generation Using an Evolutionary Algorithm. Technical report CI-127/02, University of Dortmund.

[Russel, Norvig, 1995] S. Russel, P. Norvig, 1995. Artificial Intelligence: A Modern Approach. Prentice Hall, NJ, USA.

[Siegler, 1976] R.S. Siegler, 1976. Three Aspects of Cognitive Development. Cognitive Psychology, 8, p. 481-520.

[Schlimmer, 1987] J.S. Schlimmer, 1987. Concept Acquisition Through Representational Adjustment. Technical Report 87-19. PhD thesis, Department of Information and Computer Science, University of California, Irvine, 1987. 
[SchroedI, 2005] Stefan Schroedl, 2005. An Improved Search Algorithm for Optimal Multiple-Sequence Alignment. Journal of Artificial Intelligence Research 23, p. 587-623.

[Sen, 1994] Sandip Sen, 1994. Representational effects in a simple classifier system. In Proceedings of the 1994 ACM symposium on Applied computing, p. 206-211. Phoenix, Arizona.

[Sinha, Goldberg, 2003] Abhishek Sinha, David E. Goldberg, 2003. A Survey of Hybrid Genetic and Evolutionary Algorithms. IlliGAL Report No. 2003004, University of Illinois at Urbana-Champaign, Illinois Genetic Algorithms Laboratory, urbaba, IL.

[Shin, Cho, 2003] H. Shin, S. Cho, 2003. How to Deal with Large Dataset, Class Imbalance and Binary Output in SVM based Response Model. In proceedings of the Korean Data Mining Conference, 93-107 (12 2003).

[Thrun et al., 1991] Thrun, S. B., et al. 1991. The Monk's problems: A performance comparison of different learning algorithms. Technical Report CMU-CS-91-197, Carnegie Mellon University. <http://citeseer.ist.psu.edu/thrun91monks.html>

[Tulai, Oppacher, 2004] Alexander F. Tulai, Franz Oppacher, 2004. Maintaining Diversity and Increasing the Accuracy of Classification Rules through Automatic Speciation. In proceedings of the 2004 Congress on Evolutionary Computation (CEC04), p. 2241-2249. 
[Webb, 1996] Geoffrey I. Webb, 1996. A heuristic covering algorithm has higher predictive accuracy than learning all rules. In Proceedings of Information, Statistics and Induction in Science, Melbourne, World Scientific, p. 20-30.

[Webb, Boughton, Wang, 2005] Geoffrey I. Webb, J. Boughton, Z. Wang, 2005. Not so naïve bayes: Aggregating one-dependence estimators. Journal of Machine Learning 58(1), p. 5-24.

[Whitley et al., 1997] Darrell Whitley, J. R. Beveridge, Cesar Guerra-Salcedo, C. Graves, 1997. Messy Genetic Algorithms for Subset Feature Selection. Proceedings of the Seventh International Conference on Genetic Algorithms (ICGA97).

[Witten, Frank, 2005] Ian H. Witten, Eibe Frank, 2005. Data Mining: Practical machine learning tools and techniques. 2nd Edition, Morgan Kaufmann, San Francisco.

[Yang, Honavar, 1997] Jihoon Yang, Vasant Honavar. Feature Subset Selection Using A Genetic Algorithm. Genetic Programming 1997: Proceedings of the Second Annual Conference.

[Yeung, 1991] Dit-Yan Yeung, 1991. A Neural Network Approach to Constructive Induction. In Proceedings of the $8^{\text {th }}$ International Workshop on Machine Learning, p. 228-232, Morgan Kaufmann. 
[Zhang, Ishikawa, 2004] Hong Zhang, Masumi Ishikawa, 2004. An Extended Hybrid Genetic Algorithm for Exploring a Large Search Space. In Proceedings of the second International Conference on Autonomous Robots and Agents. Palmerston North, New Zealand.

[Zhang, Jiang, Su, 2005] Harry Zhang, Liangxiao Jiang, Jiang Su, 2005. Hidden Naïve Bayes. In Proceedings of the $20^{\text {th }}$ National Conference on Artificial Intelligence (AAAI '05), p. 874-879.

[Zheng, Webb, 2000] Zijian Zheng, Geoffrey I. Webb, 2000. Lazy Learning of Bayesian Rules. Machine Learning volume 41, p. 53-84. 